\title{
An Exploration of the Relationships between Chronic Pain, Inflammation, and Herbal Medicine
}

Termeh Feinberg

Follow this and additional works at: https://researchrepository.wvu.edu/etd

\section{Recommended Citation}

Feinberg, Termeh, "An Exploration of the Relationships between Chronic Pain, Inflammation, and Herbal Medicine" (2016). Graduate Theses, Dissertations, and Problem Reports. 5585.

https://researchrepository.wvu.edu/etd/5585

This Dissertation is protected by copyright and/or related rights. It has been brought to you by the The Research Repository @ WVU with permission from the rights-holder(s). You are free to use this Dissertation in any way that is permitted by the copyright and related rights legislation that applies to your use. For other uses you must obtain permission from the rights-holder(s) directly, unless additional rights are indicated by a Creative Commons license in the record and/ or on the work itself. This Dissertation has been accepted for inclusion in WVU Graduate Theses, Dissertations, and Problem Reports collection by an authorized administrator of The Research Repository @ WVU.

For more information, please contact researchrepository@mail.wvu.edu. 
An Exploration of the Relationships between

Chronic Pain, Inflammation, and Herbal Medicine

Termeh Feinberg, MPH

Dissertation submitted to

the School of Public Health

at West Virginia University

in partial fulfillment of the requirements for the degree of

Doctor of Philosophy in Epidemiology

Kim (Karen) Innes, M.S.P.H., Ph.D., Chair

Christa Lilly, Ph.D.

Dina Jones, P.T., Ph.D.

Peter Giacobbi, Jr., Ph.D.

Gilbert Ramirez, M.P.H., Dr.P.H., CPH

Department of Epidemiology

Morgantown, West Virginia

2016

Keywords: herb, inflammation, C-reactive protein, fibromyalgia, chronic pain, and epidemiology

Copyright 2016. Termeh Feinberg, MPH 


\section{ABSTRACT \\ An Exploration of the Relationships between Chronic Pain, Inflammation, and Herbal Medicine}

\section{Termeh Feinberg}

Introduction: Inflammation is often a component of chronic pain, yet its potential role in fibromyalgia syndrome (FMS) remains inconclusive. FMS is a complex chronic pain condition affecting $2 \%$ of the population; management is challenging and treatment options remain limited. Many herbs contain antiinflammatory properties, and herbs indicated for analgesia and rheumatic conditions have traditionally been used in Appalachia. Despite the popularity of herbs in the US, determinants and patterns of herbal use with regard to pain management have not been well studied, particularly in Appalachia, where prevalence of chronic pain and related comorbid conditions is high and access to medical care is often reduced. In this series of three studies, we investigate the: 1) relation of FMS to serum C-reactive protein (CRP) in a large Appalachian population; 2) demographic, lifestyle, and health-related correlates of herbs and other complementary health approaches (CHAs) used for pain in a sample of Appalachian chronic pain patients, using a newly developed survey instrument; and, 3) the relation of herbal supplement use to FMS in two nationally representative samples of U.S. adults (NHIS 2007 and 2012), as well as trends in patterns of herbal use over time.

Methods: All participants completed comprehensive health surveys in these three cross-sectional studies. To investigate the relation between diagnosed FMS and serum levels of the proinflammatory marker CRP (Study 1), we used data on 52,535 adult Ohio Valley residents (FMS =1,125), collected in 2005-2006 as part of the C8 Health Project. Medical history, including physician diagnosis of FMS, was ascertained via self-report. To determine the correlates and patterns of herbal and other CHAs used specifically for pain in an Appalachian chronic pain population (Study 2), we collected data on 301 patients from four WV pain and rheumatology clinics using our newly developed survey instrument, the Complementary Health Approaches for Pain Survey (CHAPS) (2014-2016); correlates relating specifically to pain were measured using the Short-Form Global Pain Scale (SF-GPS). To assess the relation of diagnosed FMS to herbal supplement use (at 30 days, past 12 months, and ever) and to examine potential changes in the patterns of use over time (Study 3), we used data from the 2007 and 2012 National Health Interview Surveys (NHIS) ( $\mathrm{N}=20127$ and $\mathrm{N}=30672$ adults, respectively). Logistic and linear regression (complete-case analysis) were used to examine associations and to evaluate the potential modifying influence of gender and number of health conditions; multivariate models were adjusted for an array of demographic, lifestyle, and health factors. To account for missing data (Study 2), we also conducted additional sensitivity analyses using multiple imputation.

Results: Study 1. In this large Appalachian population, mean serum CRP was significantly higher among participants reporting a diagnosis of FMS than those without FMS $(5.54 \pm 9.8$ vs.3.75 $\pm 7.2 \mathrm{mg} / \mathrm{L}$, $\mathrm{p}<0.0001)$ ). CRP serum level showed a strong, positive association with FMS (unadjusted OR for highest vs. lowest quartile=2.5 (CI 2.1,3.0; P for trend(p<0.0001); adjustment for demographics and lifestyle factors attenuated but did not eliminate this association (adjusted odds ratio (AOR) for highest vs. lowest quartile $=1.4$, (CI 1.1, 1.6). The addition of body mass index (BMI) and comorbidities to the model further weakened the relationship between CRP and FMS (AORs, respectively, for highest vs lowest CRP quartile $=1.2$ (CI 1.0,1.4) and 1.1 (CI 0.9, 1.3), suggesting that these factors may partially explain the observed associations. Study 2: In our sample of $301 \mathrm{WV}$ chronic pain patients, $8 \%$ reported using herbs and $58.8 \%$ reported using other CHAs, including mind-body practices (28.9\%), ; acupuncture, manipulative treatments, massage, and/or movement therapies $(28.1 \%)$, and non-herbal dietary supplements (53.6\%). Herbal use in this sample was marginally, inversely associated with age (OR 
adjusted for education=0.97 (CI 0.94,1.01) and positively associated with education (OR adjusted for age $=4.94$ (CI 1.6,15.3); Herbal use also showed strong positive associations with use of other CHAs ( $(\mathrm{AOR}=11.5$ (CI 1.5,87.9); specific CHA AORs ranging from 2.4 to 10.3). Use of other CHAs was marginally, inversely associated with age (OR adjusted for education/exercise $=0.98$ (CI 0.96,1.0), and significantly and positively associated with education and physical activity (AOR's for Bachelor's+ vs. $<\mathrm{HS} / \mathrm{GED}=2.7$ (CI 1.1,6.4; Exercise per $30 \mathrm{~min}$ p/w increment = 1.14 (CI 1.01,1.3); concomitant use of other CHAs was significantly and positively associated with all $\mathrm{CHA}$ categories after adjustment for multiple confounders. Neither herbal use nor CHA use overall was associated with pain severity or use of prescription medications. Sensitivity analyses using multiple imputation did not appreciably change any observed associations. Study 3. Prevalence of diagnosed FMS was significantly higher in 2012 compared to 2007 (1.7\% vs. 1.3\%), whereas reported use of herbs declined from 57 to $41 \%$ for ever use ( $\mathrm{p}<0.0001)$. In both nationally representative samples of US adults (NHIS 2007, 2012), adults with FMS were significantly more likely to use herbs at 30 days, 12 months, or ever relative to adults without FMS; these positive associations remained highly significant even after controlling for a broad array of demographic, lifestyle, and health-related factors (Ranges: 2007 AOR's $=2.3-2.7 ; 2012$ AOR's = 1.5 - 1.6;

p's $<.0001$ ).

Conclusion: Results of these studies suggest that 1) FMS is positively associated with serum CRP, an association that may be largely explained by obesity and comorbidity, suggesting the role of inflammation in this chronic pain syndrome may be complex 2) CHA use specifically for pain in chronic pain patients in northern WV is high, and although overall reported use of herbs for pain is low, many herbs were reported by those disclosing herbal use; and 3) the use of herbs is strongly and positively associated with FMS diagnosis. Further prospective research is needed to confirm these findings, to further explore the role of inflammation in FMS to further investigate the patterns and determinants of herbal and related CHA use in FMS and other chronic pain populations, including those in Appalachia and other poor, underserved areas. 


\section{DEDICATION}

I dedicate my doctoral dissertation to my husband, Josh Feinberg. Thank you for your kindness and patience throughout this part of our lives together. Your love, strength, and firm resolve to help others continues to inspire me greatly.

\section{ACKNOWLEDGEMENTS}

I express heartfelt gratitude to my dissertation committee chair, Dr. Kim Innes, who is largely responsible for my evolution to research scientist through her continual insights, guidance, and support. I admire you greatly. Your engagement in the fields of Integrative Medicine and Epidemiology strengthen my resolve to make a difference through great research. I have always been grateful for your feedback, and truly appreciate every push to help me think more critically. Thank you.

I would also like to thank and acknowledge all other committee members for the various ways they have influenced and shaped me throughout my dissertation. Dr. Dina Jones, you have inspired me with the way you combine intellectual curiosity with your desire to help people. Your public health interventions are fueled by this passion, and you have taught me essential skills which have helped me balance organization, curiosity, and focus for my research.

Dr. Christa Lilly, you have been a cheerleader and supporter when you have not been teaching me about statistics. I am forever grateful for your open door, and hope to exemplify the kind guidance you bestow on all of your students in my career.

Dr. Peter Giacobbi and Dr. Gil Ramirez, thank you for teaching me how to think critically in the fields of Nutritional Epidemiology and Integrative Health. I appreciate your encouragement, humor, and thoughtful advice.

Additionally, I would like to thank the faculty and staff at the WVU School of Public Health, The West Virginia Clinical and Translational Sciences Institute, the Moving for Better Balance Initiative at West Virginia University, and my peers, advisors, and rotation supervisors on the WVU Behavioral and Biomedical Sciences T32 grant (NIH NIGMS T32GM081741) which enabled this dissertation; advisors and rotation supervisor include: Dr. Albert Berrebi, Dr. James Simpkins, and Dr. Jeffrey Wimsatt.

Thank you to all clinic physicians and administrators, survey participants, researchers, and field workers, for which this research would not be possible.

And, thank you to Dr. Stacie Metz (West Chester University) for changing my public health career trajectory by showing me that statistics was worthy of deep appreciation, and for mentoring me on my first research project; to Maia Toll and Andrew Celwyn, for supporting my transition from herbalism hobbyist to research scientist; and lastly, my husband, daughter, family, and friends who provided much love, patience, and laughter throughout this journey. 


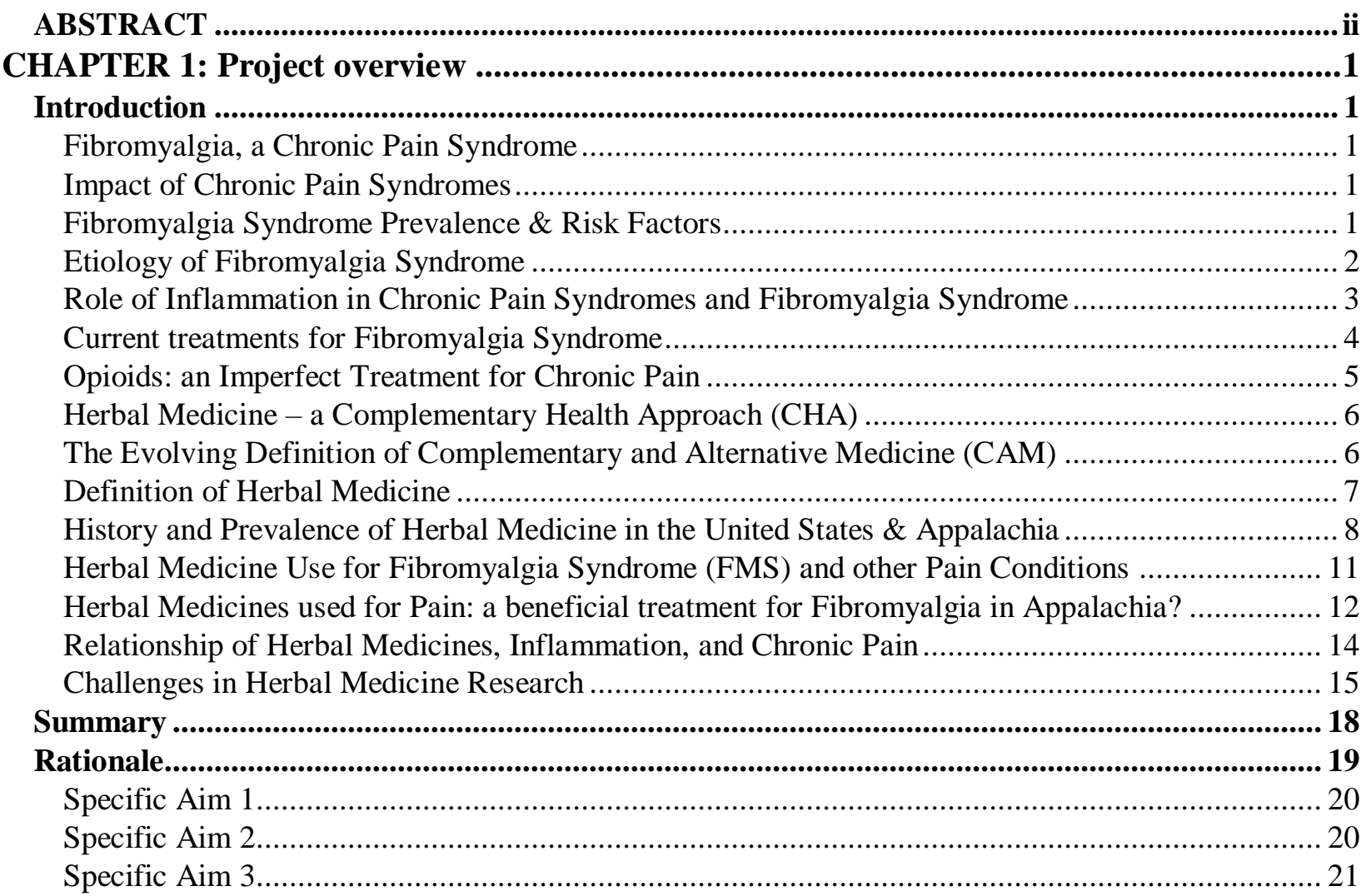

CHAPTER 2: The association of Fibromyalgia to serum C-reactive protein in a large Appalachian population ..........................................................................................22

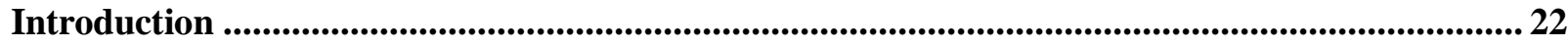

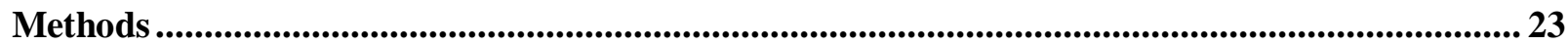

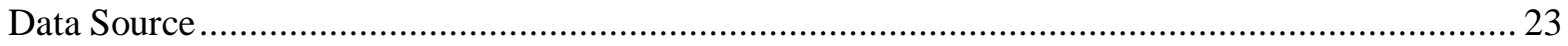

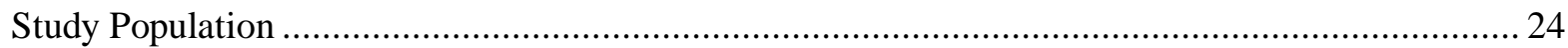

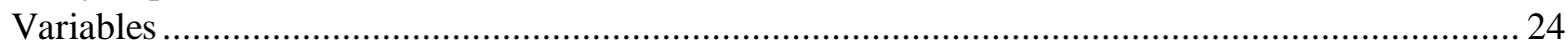

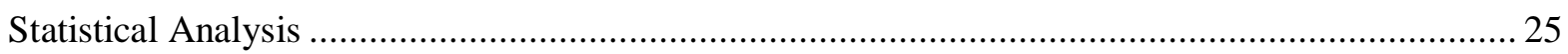

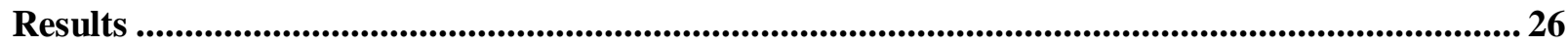

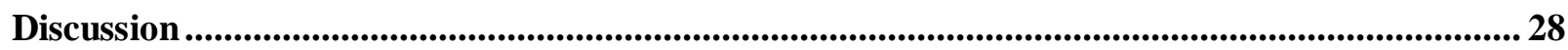

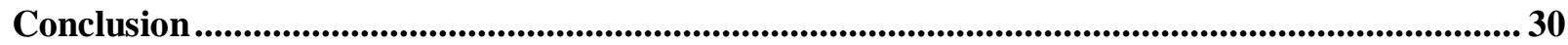

CHAPTER 3: Prevalence Trends and the Association between Herbal Supplement Use and

Fibromyalgia Syndrome in two National Samples (2007-2012) ........................................32

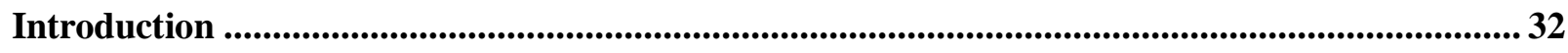

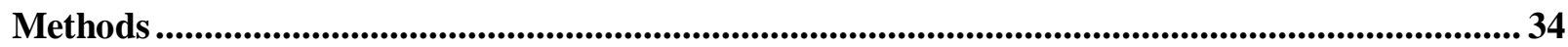

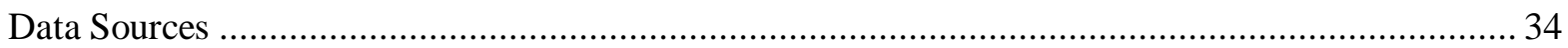

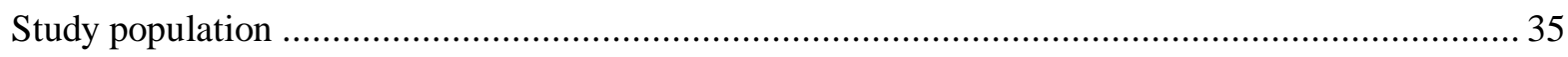

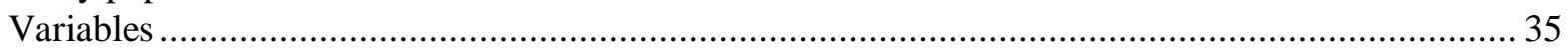

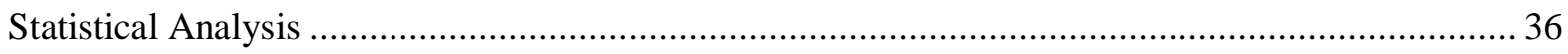

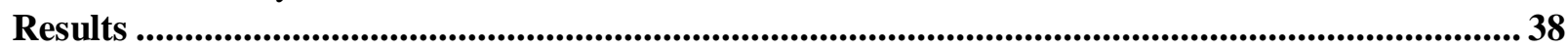

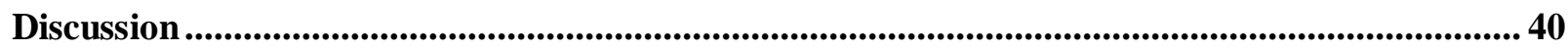

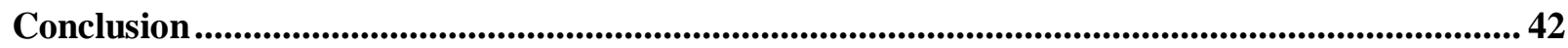




\section{CHAPTER 4: Correlates of Herbal Use and other Complementary Health Approaches}

among Appalachian Pain Patients ........................................................................................................43

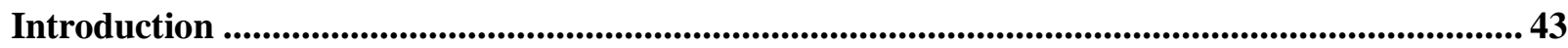

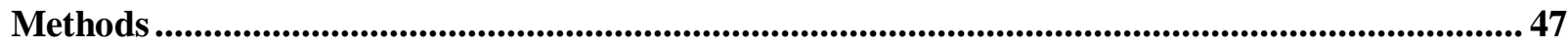

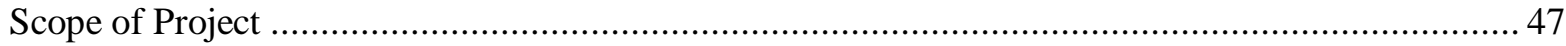

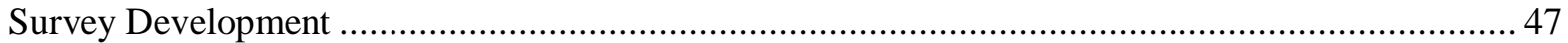

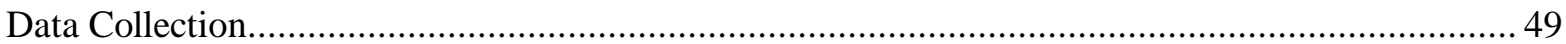

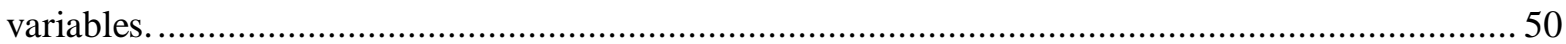

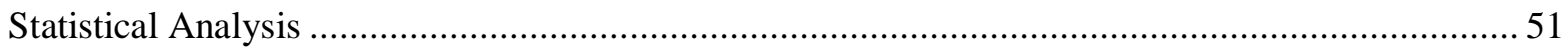

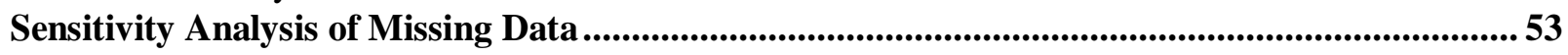

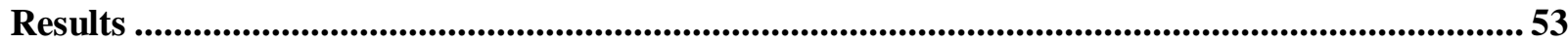

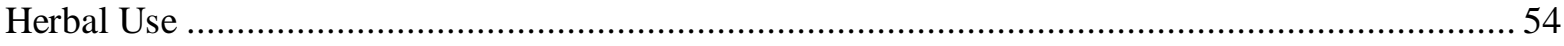

Other CHA use

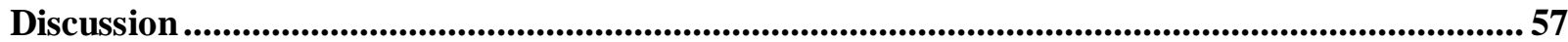

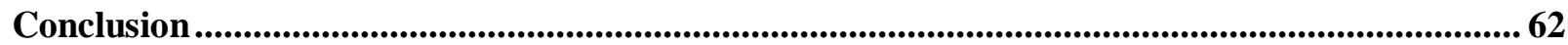

CHAPTER 5: Discussion .....................................................................................................62

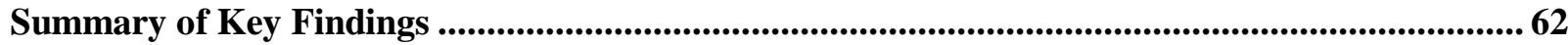

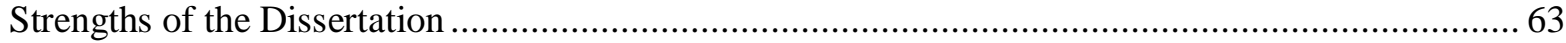

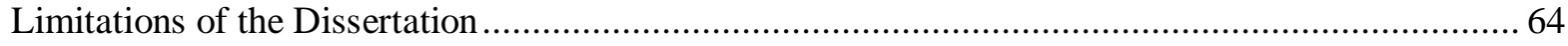

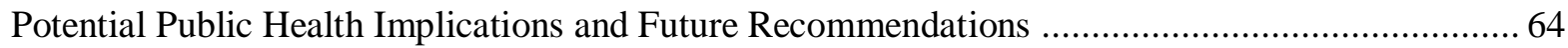

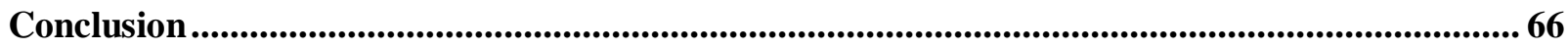

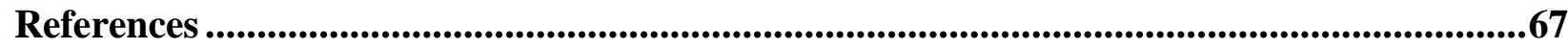

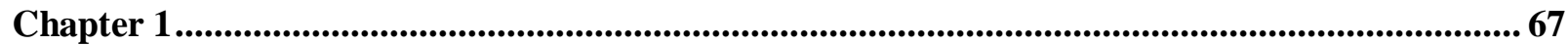

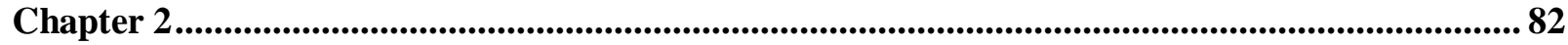

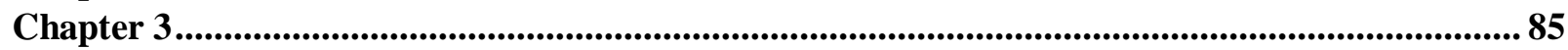

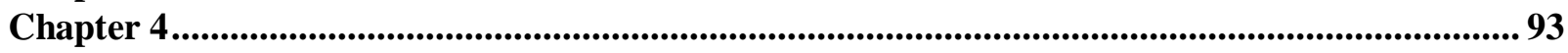

Chapter 5.............................................................................................................................................. 100

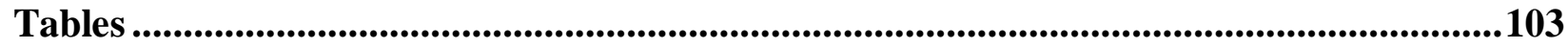

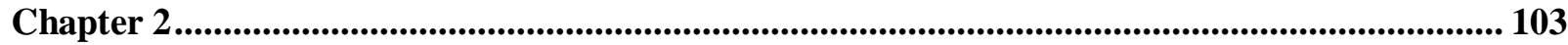

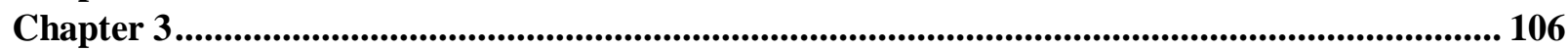

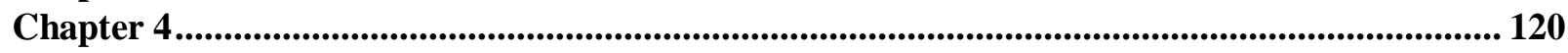

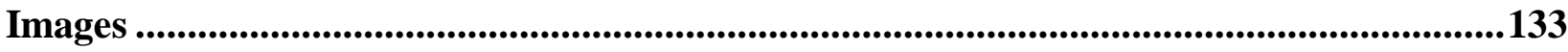

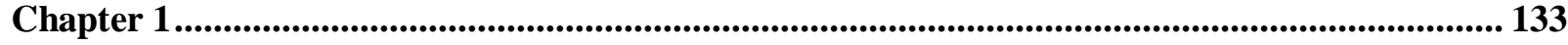

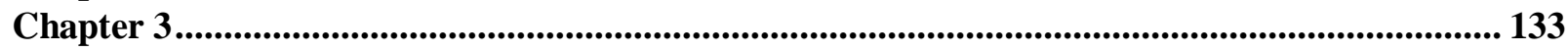

Figures.................................................................................................................................134

Chapter 1................................................................................................................................................ 134

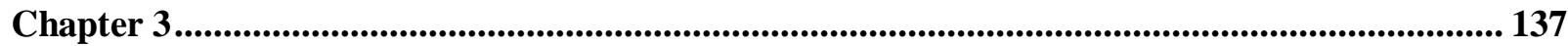

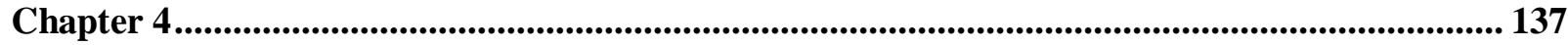

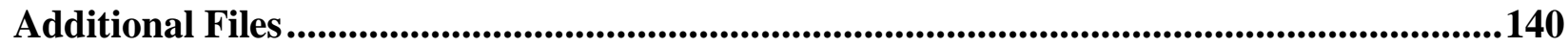

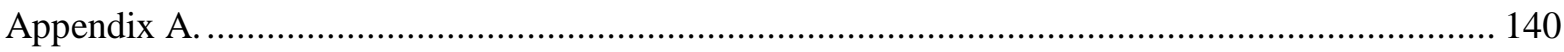

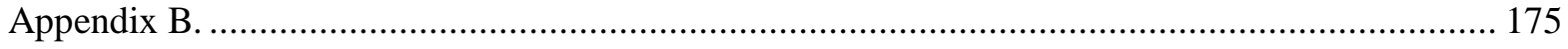

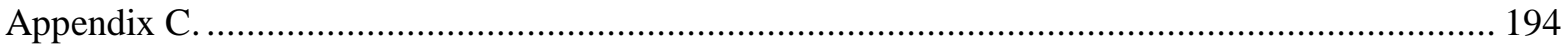

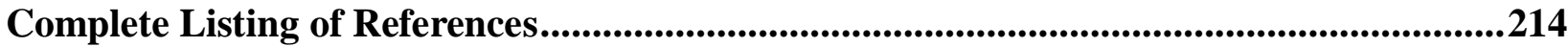




\section{CHAPTER 1: Project overview}

Introduction

\section{Fibromyalgia, a Chronic Pain Syndrome}

\section{Impact of Chronic Pain Syndromes}

Chronic pain, defined as ongoing or recurrent pain, lasts beyond the usual course of acute illness or injury for more than three to six months, and is both debilitating and costly. Over $20 \%$ of adults may eventually experience chronic pain (1-3), and as many as 100 million individuals are affected in the United States alone (4). The effects of pain exact tremendous health care costs, rehabilitation and lost worker productivity, as well as the emotional and financial burden placed on patients and their families. The costs of unrelieved pain result in longer hospital stays, increased rates of re-hospitalization, increased outpatient visits, and decreased ability to function fully leading to lost income and insurance coverage; unrelieved chronic pain problems often lead to work disability and maintenance of health insurance (5). Chronic pain costs the U.S. more than $\$ 635$ billion annually in medical costs and lost productivity, which equates to approximately $\$ 2,000$ per person in the U.S. The Institute of Medicine stresses the importance of increased research into the translation of effective treatments into practice and into the distribution, prevalence, and burden of pain (6).

Fibromyalgia Syndrome Prevalence \& Risk Factors

Fibromyalgia syndrome (FMS) is a non-fatal rheumatologic chronic pain syndrome affecting approximately $0.5-5 \%$ of populations in developed countries (7), including $1.75 \%$ of those in the U.S. (8). FMS is a "central" pain syndrome characterized by a constellation of somatic symptoms that are typically present in addition to widespread pain (e.g., fatigue, sleep disturbance, memory, and mood problems) (912). FMS is also characterized by morning stiffness, headaches, and increased sensitivity to loud noises, bright lights, and temperature extremes; women with FMS often report painful menstrual periods (13). Although FMS affects both sexes and people of all ages, the majority (80-90\%) have been Caucasian $(13,14)$ middle-aged women $(13)$. Family members of FMS patients are at a higher risk for FMS (15); the cause is unknown. 
Between 80 and 90\% of people diagnosed with FMS are middle-aged women, although FMS can affect both sexes and people of all ages (13). FMS is similar to many regional pain syndromes in that peripheral or mechanical factors do not contribute, or contribute only minimally to the widespread chronic pain typifying FMS; standard laboratory tests fail to reveal a physiologic reason for the pain experienced (16). The cause of FMS is still poorly understood, and there are currently no diagnostic laboratory tests for FMS, with diagnosis typically based on distribution of pain (with at least 11 of 18 painful 'tenderpoints' for at least 3 months, according to 1990 American College of Rheumatology Diagnostic Criteria (17), and, updated 2010 diagnostic criteria incorporating quality-of-life impact of FMS(17)) and exclusion of other, competing causes of pain. Because there are no established objective tests, and very limited management options for FMS, some doctors may conclude a patient's pain is not real, or they may tell the patient there is little they can do (18).

\section{Etiology of Fibromyalgia Syndrome}

While the etiology of FMS is unknown, there may be a number of factors involved. Many people associate the development of FMS with a physically or emotionally stressful or traumatic event, such as an automobile accident or repetitive injuries, while some link it to another illness (11). FMS occurs spontaneously in others, and limited research has investigated if it may be caused by a virus that changes the way the brain perceives pain (11). People with FMS may have abnormal spinal fluid levels of Substance $\mathrm{P}$, a chemical which helps to transmit and amplify pain signals to and from the brain (11). Despite the potential variation in underlying cause(s), however, it is clear that people with FMS have an increased sensitivity to pain. Because genes regulate the way the body processes painful stimuli, people with FMS may have a gene or genes that cause them to react strongly to stimuli that most people would not perceive as painful (19). Several genes have been identified to occur more commonly in FMS patients; studies indicate that women who have a family member with FMS are more likely to have FMS themselves; several cerebrospinal MircoRNAs were recently identified in FMS cases in a small casecontrol study (20). However, it is unknown if heredity is solely responsible, or if shared environmental factors play a role as well. Further research on the perception of pain and related variables among people with FMS is needed.

Like arthritis, FMS is considered a rheumatic condition, a medical condition that impairs the joints and/or soft tissues and causes chronic pain; individuals with higher BMI (21), autoimmune conditions (22), osteoarthritis (23), kidney (24), respiratory (25), cardiovascular (24), liver (26), and endocrine (24) disease, diabetes (27), severe allergies (24) and sinus disease (28), stomach conditions (24, 26), and headache (29) may be more likely to have FMS. Those with FMS are also more likely to have 
two or more coexisting pain conditions in addition to FMS, suggesting the plausibility of a common underlying cause; however, shared mechanisms between comorbid conditions and FMS have remained largely unexplored (13).

\section{$\underline{\text { Role of Inflammation in Chronic Pain Syndromes and Fibromyalgia Syndrome }}$}

Chronic pain remains an unresolved problem in human medicine which greatly impairs quality of life and prolongs treatment. Developing and identifying effective therapies is difficult due to the array of mechanisms and signaling pathways through which chronic pain may be generated (30, 31). A common underlying mechanism of chronic pain is the presence of inflammation at the site of the damaged or affected tissue (31), and inflammatory responses in the peripheral and central nervous systems play key roles (32). The release of pro-inflammatory and immunoactive substances such as cytokines (which are chemical messengers and can also be anti-inflammatory), neurotrophic factors, and chemokines initiate local actions and can result in a more generalized immune response by directly activating nociceptive sensory neurons, leading to or promoting the generation of prolonged pathologic pain (33), and, ultimately, to a persistent chronic pain condition (31).

Certain inflammatory cytokines are also involved in nerve-injury/inflammation-induced central sensitization, and are related to the development of contralateral hyperalgesia (an extreme, exaggerated reaction to a stimulus) and allodynia (central pain sensitization following painful, often repetitive stimulation) (33). FMS is a condition that typically causes hyperalgesia and allodynia (34).

Although inflammation, as a characteristic reaction of tissues to injury or disease marked by physical swelling, redness, heat, and pain, is not a classical symptom of FMS (18), further investigation is necessary to rule out the existence of chronic, low-level inflammation among people with FMS. Currently, cytokines are being investigated by the National Institutes of Health (NIH) in lab/pre-clinical studies to determine the extent to which chronic pain in FMS patients is associated with the activation of cells in the nervous system (18). The possible link between systemic inflammation and FMS must be investigated since existing studies suffer from limited generalizability and potential misclassification bias. Some of these investigations $(35-49)$, but not others $(43,50)$ reported increased likelihood of FMS among those with elevated levels of various pro-inflammatory indicators. However, only five of these studies measured C-reactive protein (CRP) $(44,47-49,51)$, which is a marker of inflammation that is a commonly ordered lab workup among patients whose symptoms may indicate the presence of FMS (16); Four suggested a positive association between CRP and FMS (44, 47-49). In addition, interpretation of findings in these studies is hindered by small sample sizes. To date, only one large, cross-sectional, populationbased study has examined the association of CRP to FMS (48). The study excluded women, and combined FMS with other pain syndromes, rendering assessment of the specific relationship between CRP and FMS 
difficult. Thus, the role of inflammatory mediators in FMS also needs to be elucidated by larger, population-based studies.

\section{Current treatments for Fibromyalgia Syndrome}

Family physicians, general internists, rheumatologists, and some pain clinics treat those who have FMS (13). However, not all physicians are familiar with FMS and its treatment. Therefore, some FMS patients experience difficulty in receiving diagnosis and consistent care for their symptoms. Treatment for FMS and other chronic pain conditions is often poorly handled.

Although there is 'gold' level evidence (Cochrane) that supervised aerobic exercise training has beneficial effects on physical capacity and FMS symptoms (52), and some evidence that cognitive behavioral therapy may provide a small incremental benefit in reducing pain, negative mood and disability at the end of FMS symptom treatment and at long-term follow-up (53) (Appendix A), people with FMS are typically treated with pain medicines, antidepressants (54), muscle relaxants, and sleep medicines (11). Often, neuromodulators (substances which alter the way nerves communicate with each other, affecting the overall activity level of the brain, potentially reducing the amount of pain (55)) and anti-epileptics are used for treating FMS. These and other drugs used to treat FMS carry significant side effects and include Gabapentin (56), Fluoxetine (57), Pramipexole (57), Amitriptyline (54), Duloxetine (58, 59), and Tramadol plus Paracetamol (57). Although three drugs (Lyrica, Cymbalta, and Savella) have been approved by the FDA for treatment, they also carry significant side effects $(57,60,61)$, and the mechanism by which these drugs produce their effects is unknown. For some FMS patients, these drugs are not effective (11). Nonsteroidal Anti-Inflammatory Drugs (NSAIDs) inhibit prostaglandins, which play a role in pain and inflammation; these are given as treatment for FMS symptoms (18), but carry significant side effects for as many as $25 \%$ of long-term users (62).

For a subset of people with FMS, narcotic medications are prescribed for severe muscle pain. However, concrete evidence is lacking regarding the efficacy of narcotics for the management of chronic pain for the majority of those with FMS. Many doctors hesitate to prescribe narcotics for long-term use because of the potential that the person taking them will become physically or psychologically dependent on them (18). However, more than four-fifths of FMS patients receive opioid therapy (63); Because specific recommended treatments for this chronic disease are highly variable, a consensus on effective treatment for the disease is not known (64-66). Pharmaceuticals used for other diseases may be prescribed as treatment for FMS, but there is limited quality research to support this practice, particularly regarding Phenytoin (67), Clonazepam (68), Carbamazepine (61, 69), Topiramate (70), Pirlindole (71), Valproate $(61,72)$, Lamotrigine $(61,73)$, Lacosamide $(61,74)$, and lastly, the narcotic Oxycodone (75). 
Complementary Health Approaches (CHA) are often used by those with chronic pain. Further, the American College of Rheumatology suggests that people with FMS consider non-drug treatments in addition to their pharmaceutical approaches, and that certain CHAs may be helpful in managing FMS symptoms. However, many of these treatments, including herbal dietary supplements, have not been well tested in FMS patients (76-78).

While Mind-Body therapies (a group of CHAs such as meditation, hypnotherapy, relaxation, and guided imagery) may improve self-efficacy, quality of life, and pain for people with FMS, systematic reviews and meta-analyses on other treatments for FMS indicate a need for further research in the areas of acupuncture (79), multidisciplinary rehabilitation for musculoskeletal disorders (a commonly used intervention) (80), resistance training (81), and traditional Chinese medicine (82). We attempted to conduct a meta-analysis in December 2014 to assess if herbal use (including Eastern or Western) was simply a placebo effect for FMS treatment. The inclusion criteria were randomized clinical trials only including FMS patients, compared herbs with placebo or pharmacological therapies, and included clinical outcomes assessing FMS symptom severity and/or pain and/or quality of life. However, no trials met this criteria, indicating a lack of research assessing the potential impact of herbs on FMS.

\section{Opioids: an Imperfect Treatment for Chronic Pain}

Over the past decade, the worrisome trend of increased prescription and utilization of opioids for the treatment of chronic nonmalignant pain, including FMS, has emerged (83). Opioid pain relieversalso called prescription painkillers - such as Oxycodone, Hydrocodone, Fentanyl, and Hydromorphone, were responsible for three-fourths of all prescription drug overdose deaths in the United States in 2010 (84). Despite recommendations against the use of opioids for the treatment of FMS, evidence suggests widespread and increasing clinical utilization (85). Recent evidence shows that over four-fifths of FMS patients receive opioid therapy and that treatment using approved medications not only does not reduce this trend but also tends to increase opioid utilization rates (63). Nationally, deaths involving opioids (using age-adjusted data) have more than quadrupled since 1999 (84). West Virginia ranks among the worst states for its health disparities, especially for opioid pain medication misuse and abuse. West Virginia's overdose death rate for 2010 (28.9 per 100,000 population) was more than double the national rate (12.4 per 100,000 population), which has also been climbing over time (86).

Opioid treatment for chronic pain management presents a great challenge in the unique demographic of Appalachia. Many chronic pain patients experience poor treatment outcomes (2). Chronic and malignant pain is difficult to manage, partially due to limited efficacy and negative side effects from conventional medical therapies, but also because some physicians are reluctant to prescribe opioids (6), while others overprescribe them (85). West Virginia has the third highest national ranking for prescription opioid use 
in FMS patients ( 19.4\%) (87); many patients become dependent on opioids, and opioid use and misuse remain an important topic in West Virginia (86).

Understanding the usage and efficacy of other, safer forms of chronic pain treatment are necessary, and may include CHAs. CHA use exists in chronic pain patient populations (88-94), and encompasses a variety of therapeutic modalities not customarily prescribed by medical doctors to treat chronic pain, such as natural products (e.g., herbs, dietary supplements, etc.) and mind and body practices (e.g., yoga, meditation, etc.), as well as the practices of traditional healers, Ayurvedic medicine, Traditional Chinese Medicine, homeopathy, and naturopathy, among other non-mainstream approaches. Most people using CHA approaches use them along with conventional treatments $(90,95,96)$, and patients often do not disclose the use of CHAs to their physicians $(97,98)$; the appropriate role for CHAs in the primary care of chronic pain remains controversial. Physicians disagree with one another and with their patients about the cost, validity, and effectiveness of individual CHAs (99). CHA use may reflect greater autonomy among a population of patients who wish to direct their own health care and are less dependent upon conventional medicine (88), with patients choosing CHAs because of concerns over the safety of conventional medicine (100).

Given the continuing rise in fatalities due to opioid misuse, expanding treatment options for pain is a necessary step in reversing the explosion of deaths on a national- and a state-level for WV. A deeper understanding of the relationship between analgesic CHAs (such as herbs), which may be safer and more well-tolerated than opioids and current pharmaceutical treatments, and the role of inflammation affected by those potential treatments, is warranted.

\section{Herbal Medicine - a Complementary Health Approach (CHA)}

\section{The Evolving Definition of Complementary and Alternative Medicine (CAM)}

More than 30 percent of adults and about 12 percent of children use health care approaches developed outside of mainstream Western, or conventional, medicine (88). Surveys have yielded differing conclusions in explaining the increasing popularity of these approaches $(88,89,101-103)$. When describing these approaches, people often use the terms "alternative" (If a non-mainstream practice is used in place of conventional medicine) and "complementary" (if a non-mainstream practice is used together with conventional medicine), and these various approaches (called "therapies" or "modalities" in previous literature) have, until recently, been called "CAM" (Complementary and Alternative Medicine). However, true alternative medicine is uncommon, as most people who use nonmainstream approaches use them along with conventional treatments. Therefore, the National Center for Complimentary and 
Integrative Medicine (NCCIH), a division of the National Institutes of Health (NIH), recently suggested the use of the umbrella term "Complementary Health Approaches" in discussing practices and products of non-mainstream origin, and "Integrative Health" when talking about incorporating complementary approaches into mainstream health care (104). 'CAM', therefore, will henceforth be considered "Complementary Health Approaches" (CHA) in this dissertation. Examples of CHA approaches include (but are not limited to) acupuncture, aromatherapy, biofeedback, chiropractic care, energy healing, folk remedies, herbal medicine, homeopathy, hypnosis, imagery and relaxation techniques, lifestyle diet, massage, megavitamins, naturopathy, Reiki, copper bracelets, and yoga $(105,106)$.

In Integrative Health and self-prompted use of CHAs, treatments are individualized for each patient, and when a practitioner is present, treatments may be individualized for each patient at each session (107). Sufficient anecdotal evidence suggests that many folk cures do produce their anticipated result (108), warranting additional research regarding the efficacy of these treatments. It is clear that CHA usage is popular; in 2007 , about $\$ 14.8$ billion was spent on the purchase of non-vitamin, nonmineral, natural CHA products in the United States (109). According to the 2012 National Health Interview Survey (NHIS), which included a comprehensive questionnaire regarding the use of CHAs by Americans, $17.7 \%$ of American adults reported consuming dietary supplements other than vitamins and minerals in the past year, making these products the most popular CHA used in the U.S. (104).

Compared with nonusers, CHA users tend to be women, white, middle-aged, of higher income, and better-educated (90, 106, 110-116). The use of CHA approaches is higher for chronic conditions than for acute conditions $(112,116)$, and users are more likely to be wellness-oriented $(116,117)$, with the primary use for health promotion $(89,90,116,118)$.

\section{Definition of Herbal Medicine}

Herbs (plant-derived products, also known as 'Botanicals', 'Phytotherapies', 'Phytomedicine', and some 'biologically based therapies') are sometimes used to affect health status through biological activity within the body, and are most often sold as dietary supplements. Herbs are part of the broader CHA group of approaches called 'Natural Products', which also include vitamins and minerals (which are not plant-derived). The use of herbs for potential health benefit is sometimes called 'Herbal medicine' and is part of many 'Folk Medicine' traditions indigenous to specific populations around the world.

Herbs are available for use as supplements, tinctures (alcoholic and non-alcoholic extracts), loose herbs for making teas/infusions/decoctions, and are sometimes prepared as salves or ointments for topical use $(119,120)$. Some traditional uses indicate the smoking of herbs for health benefit. However, this dissertation does not specifically include the smoking of herbs and will avoid specific investigation of the 
herb Marijuana (Cannabis sativa, indica, and ruderalis) in this proposed project, due to pending legality issues in the U.S. involving its growth, distribution, and use for recreational and health purposes. It is possible that this omission may result in the under-ascertainment of herbal use prevalence. In this research project, herbs will be referred to as "herbs", "herbal supplements", or "herbal medicine" (in lieu of "herbal remedies"), depending on the specific aim of the project.

Herbal medicine is also used as a complement to or as a component of other CHA approaches, including Ayurveda, folk medicine or traditional healing, naturopathy, Traditional Chinese Medicine, and to a lesser degree, specialized diets such as the macrobiotic diet. However, because existing research does not commonly discuss herbal use as a component of other CHA modalities, our aims will utilize and focus on research which relates specifically to the definition set forth by the Center for Disease Control (CDC) National Center for Health Statistics for 'Nonvitamin, nonmineral dietary supplements'; specifically, the latter are defined as herbs or other nonvitamin supplements (including those in pill, capsule, tablet, liquid or other form) that have been labeled as dietary supplements. Whenever discrimination is possible, we will limit our analyses to include only plant-based products (the NIH definition of 'herbs').

Because of the historical value placed on the sharing of herbal knowledge through 'Folklore' and 'Folk Medicine' in Appalachia (defined as a tradition of treating sickness by herbs, ethnic foods, prayers, healing objects, magic and dance ceremonies (121)), these terms will be considered as herbal medicine use in this project whenever it is clear that herbs were part of a healing regimen, to limit possible misclassification bias. Plant materials used for making folk medicines include the roots, bark, wood, sap, and leaves of plants (122).

Many of the top-selling herbs in the US have been subjected to clinical trials in Europe and are currently recognized as beneficial for a range of health conditions by respected international health authorities, including the World Health Organization, The European Scientific Cooperative on Phytomedicine and the German Commission E. (123). However, the vast majority of herbs and their components have yet to be studied; even among relatively well-researched herbs, specific active components have yet to be identified, and potential interactions with other constituents of the herb, other herbs, and pharmaceuticals remain largely unexplored. The US Food and Drug Administration (FDA) does not regulate herbal products, and frequently, herbal products contain adulterants and manufacturers make unsubstantiated (and sometimes illegal) claims regarding specific health benefits. However, herbs remain a popular tool for the self-management of health among many people.

History and Prevalence of Herbal Medicine in the United States \& Appalachia 
Although herbal medicines are considered a CHA in the West, herbal medicine remains a primary source of medicine in many parts of the world, including present day China, India, and many countries in South America and Africa (123). Since aboriginal times, more than one thousand plants have been used for medicines in the United States and Canada. Native American exchanges with European settlers were important contributions to folk medicine (122), and both groups believed that a plant existed for every malady known to humankind (124). In the 1990s, specifically, Complementary and Alternative Medicine ('CAM', the term used before CHA) gained increasing popularity in the United States $(90,91,106,125$, 126). The prevalence of adults using herbs increased from $2.5 \%$ in 1990 to $12.1 \%$ in $1997(106,125)$ in some of the earliest U.S. prevalence studies. Several groups also reported relatively high rates of herb and supplement use, including women $(125,127-135)$, middle aged adults $(125,128,134)$, Caucasians $(125$, $128,129,132,133)$, Asians $(125,130,136)$, and Latinos [106]. One study showed higher herbal supplement use among minority populations (127).

Because we observed varied prevalence rates for herbal use, we conducted a preliminary systematic literature review (Appendix C) assessing the herbal use in the United States general population; our review was restricted to large studies $(\mathrm{N} \geq 500)$ of adults aged at least 18 years and published between 2001-2012, and we excluded studies assessing specific conditions such as diabetes and cardiovascular disease, among others. A total of 32 eligible studies were identified for inclusion. Collectively findings indicated that rates of medicinal herb use averaged $18.1 \%$, ranging from $\sim 5 \%$ in a group of physicians (137) to $38.5 \%$ in a sample of Asian Americans (138). African Americans reported the lowest rates of herbal use (15.3\%), followed by Hispanics (19.4\%) and Non-Hispanic Whites (19.9\%) $(109,116,118,137-165)$. Overall mean rates of herbal use were similar to the national prevalence rates for nonvitamin, nonmineral dietary supplement consumption cited in 2002 (18.9\%), 2007 (17.7\%), and 2012 (17.7\%); together, use of herbs and other supplements remains the most popular complementary health approach in the U.S (166). Other gaps identified included a botanical use classification as either too narrow or too broad to enable generalizability of results, in addition to confusing presentation of results. Commonly cited suggestions for future studies included drug/botanical interactions, further investigation of the concurrent utilization of other CHAs, and increased understanding of beliefs and experiences which may act as enabling factors for herbal use.

Appalachia is a mountainous region in the eastern United States spanning southern New York to northern Mississippi, including all of West Virginia and parts of 12 other states (167). It is one of the most botanically diverse regions in the world (124); Appalachia's many micro-habitats are home to some 2,500 plant species, of which some 1,100 have been reported to have medicinal properties (168). As recent as the 1990s, there was written anecdotal evidence of medicinal plant and home remedy use in the Appalachian region, particularly among the elderly population (169). These plants have a rich tradition in oral history and folklore; while some plants are well-known only from older accounts, others have been 
and continue to be used in the preparation of pharmaceuticals. Digging for the herbs Ginseng (also known as "sangin" or "digging sang"), wild cherry, goldenseal, sassafras (124), and other medicinal herbs was noted as a general occupation as early as 1886 (170-172), and prior to WWII was a vital part of the local economy of Southern Appalachia (124); several of these herbs continue to be harvested in WV (Image 1) (120, 122, 173-178).

The Appalachian region remained the principal primary source of crude drugs (i.e., raw plant material) (124) until the 1980s (179) and the advent of synthetic drugs (124); Appalachian forests continue to provide the primary domestic source of wild native plants used in the American drug industry. Much of the value of the plants harvested and used in the Appalachian region remain largely unknown and often undocumented. However, much of the oral history of the area is destined to be irretrievably lost to future generations unless it is collected in the very near future $(121,179)$. Rates of herbal use in the Appalachian region have never, to our knowledge, been investigated, with the exception of one study in 2523 older North Carolina adults which reported a prevalence rate of 19.3\% (141).

Historically, people living in remote, isolated regions of southeastern KY found it necessary to formulate their own medicines, cures, and treatments for their self-diagnosed ills because of the scarcity and inaccessibility of trained physicians, nurses, and hospitals (179). Most families kept a tonic on hand for whenever someone felt "puny" (sluggish, weak), also known as the folk illness "low blood" (124) (Figure 1). When engaging in research to assess herbal use, it is important for investigators to be aware of self-diagnosed health conditions that may be part of folk illness tradition, rather than evidence-based diagnoses made by physicians. Because herbs were traditionally self-administered, people may continue to self-diagnose based upon folk illnesses; there has been contemporary practice of several widespread and culturally significant systems of "traditional" or "folk" medicine in eastern regions of NC and VA (108), and anecdotal evidence hints at its existence as far south as Alabama and potentially as far north as West Virginia. A single plant, such as Sarsaparilla or Sassafras, could serve as a health tonic for some of these folk illnesses, but often several plants were used (124). Qualitative analyses of patient sessions with herbalists, lay midwives, and "root doctors" (who formed part of a traditional ethnomedical system utilizing plant roots among African Americans, which in turn, influenced health behaviors among white and Native Americans in the rural South) in various rural counties in the central Piedmont and eastern NC from 1978-1991 demonstrated that individuals generally agreed herbal remedies worked effectively to cure most minor, "natural" ailments, which are a type of folk disorder (Figure 1). Qualitative research on folk medicine in Appalachia indicates a current revival of herbal medicine usage (Figure 2) $(121,180)$. However, aside from a few very limited studies on specific patient populations, distributions, patterns, and correlates have yet to be determined for herbal use in this particular region.

Physical barriers, as well as social and economic distinctions, have played a role in historical Appalachian herbal usage. Herbal consumption by Appalachian populations has varied depending on the 
history and place; types of herbs consumed has largely derived from European and American herbal texts of the nineteenth century, which were likely learned through European and Native American tradition $(179,181)$. Herbal medicine appeared to be unevenly distributed in a North Carolina population study, with one or two practicing herbalists in any given rural community (1978-1991). Herbalists still actively practicing may treat hundreds or even thousands of people a year (182). Traditional herbalists have many years of experience in collecting and preparing plants, know the properties of many different varieties and species, and observe over time the empirical effects of their remedies on different illnesses. Traditional herbalism, however, is a skill that appears to be dying out as existing practitioners grow older. Traditional herbalists often understand the "proper" ways to prepare plants, and as their knowledge is lost with time, the likelihood of negative side effects and toxicity among users may increase (108).

Although the landscape of healthcare has evolved through time, the rurality of the Appalachian region, particularly in $\mathrm{WV}$, may continue to contribute to the usage of herbal medicine. Isolation from hospitals, doctors, and pharmacies have, and to some extent, continued to characterize many populations resident in Appalachia today (122). It is not surprising that people facing these conditions of rurality may rely on traditional remedies such as herbs, and/or folk practitioners, many of whom are both accessible and willing to offer advice or treatment on short notice, often in their own homes (182).

Prevalence estimates of CHA use, including herbal use in WV, are largely unknown; a crosssectional study among primary care clinics from nearly three decades ago $(\mathrm{N}=170)$ found that $73 \%$ of patients used one or more folk medicine remedies, which are a CHA including herbs (mean= 4.5 remedies) (183). A more recent cross-sectional study on pregnant $\mathrm{WV}$ obstetrics patients $(\mathrm{N}=578)$ showed $45.2 \%$ of the participants used herbal medications, with acquisition from a plethora of retailers: grocery stores (40\%), discount stores (21.8\%), mail-order catalogs (11.3\%), herbalists (7.0\%), in-home sales by friends and fellow church members (4.0\%), and pharmacies (3.8\%). Two percent of participants grew and harvested their own herbal medicines (184). In lieu of largely absent prevalence rates for herbal use in Appalachia, there is anecdotal evidence of Appalachian (185), specifically WV, herbal usage in anthropological literature (175, 186-190). Within the last 40 years, WV was documented as having an unusually strong tradition of folk medicine (191).

\section{Herbal Medicine Use for Fibromyalgia Syndrome (FMS) and other Pain Conditions}

Pain-related disorders are the most commonly reported conditions for which patients use CHA approaches $(86,88,93,105,106)$. A systematic review of the prevalence of CHA use in international rheumatology patients, specifically FMS patients, indicated about two-thirds of patients who had FMS were using at least one type of CHA by 1998 (192). Among the mixed study designs, three UK studies indicated that 20\% $(\mathrm{N}=78)(193,194)$ and 35\% $(\mathrm{N}=94)(194,195)$ of Rheumatoid Arthritis patients used 
herbal medicines, while $78 \%(\mathrm{~N}=90)(194,196)$ of FMS patients used dietary supplements (including the use of herbal supplements).

Results of a 2002 US national survey suggest that bodily pain, chronic conditions, and functional impairment are associated with the use of CHAs among midlife and younger adults (156). The same survey showed 3.3\% of those with Arthritis, Gout, Lupus, and FMS used natural products (160). Another study utilizing the same survey showed that those who worked less than full time were more likely to use herbs compared with those who worked full time or more (116), which may indicate support for our hypothesis that people with disabling diseases which keep them from working full time, such as FMS, have an increased utilization of herbal supplements.

A U.S. study of CHA approaches among primary care patients receiving opioids ( $\mathrm{N}=908)$ as a primary treatment method for chronic pain showed that $6.8 \%$ used herbs and supplements, and that $44 \%$ had used at least one CHA approach in the past year. CHA utilization was significantly related to age, female gender, pain severity, income, pain diagnosis of neck and upper back pain, and illicit drug use (93). Twenty-one percent of FMS cases used opioids, as well as herbs and dietary supplements (which were not plant-derived) $42.9 \%$ (mean $=2.8$ per person) of the time, compared to $23 \%$ (mean $=2.2$ per person) of the controls in a national case-control study (FMS $=434$, Control $=198)$. Authors found no evidence of FMS status being associated with any specific herb. This was a moderately strong study, but limitations included lack of a random sample/significant differences on all demographic variables between cases and controls, self-reported data, and a low sample size for controls using herbs, severely limiting the statistical ability to compare herbal use between the two groups. Herbs used most often by those with FMS were Ginkgo (3.7\%), Garlic (3.0\%), Ginseng (3.0\%), Echinacea (2.5\%), Evening Primrose oil (2.3\%), and Valerian (2.3\%) (197). Although a number of studies have examined general herbal use among specific populations, no population studies have asked pain patients about herbs used specifically for pain. Additionally, whether opioids can be reduced by introducing herbal medications remains to be studied.

Herbal Medicines used for Pain: a beneficial treatment for Fibromyalgia in Appalachia?

Pain-related disorders are the most commonly reported conditions for which patients use CHAs $(86,104-106,198)$. The history of Willow (Salix spp.) and other salicin-rich plants, which were precursors to Aspirin, is an example of the role botanicals have played in the management of pain (123). A meta-analysis of Capsicum (a compound extracted from the fruit of Chili peppers $C$. frutecens and others) for the treatment of pain, reported that topical Capsaicin was superior to placebo for the treatment of chronic pain due to neuropathic and musculoskeletal disorders (with trials of moderate to high quality); 
the FDA has approved Capsaicin as an over-the-counter topical cream (199). There is a great deal of historical evidence which reveals extensive use of botanicals for the relief of pain, and plants with analgesic, anti-inflammatory and/or anti-spasmodic activities continue to be used (123). However, there is a need for research regarding the use and efficacy of herbs that may benefit those with FMS and its comorbidities, especially in the Appalachian region, where opioid prescription drug misuse and abuse have skyrocketed, and safer, effective treatments aside from other existing pharmacological therapies for those with FMS are clearly needed.

Existing Cochrane reviews and meta-analyses regarding herbal efficacy for chronic pain syndromes often co-occurring with or similar to FMS (Appendix A) include Passiflora (200), Valerian (201), and Kava (202) for anxiety disorders, compounds Qishe and Extractum Nucis Vomicae for chronic neck pain (203), Feverfew for preventing migraine (204-206), St. John's Wort for major depression (207), and mixed herbal medicine(s) for low back pain (208), irritable bowel syndrome (209), and rheumatoid arthritis (210) (unless otherwise noted, Latin botanical names are omitted in this paper when source identification is unclear; otherwise, they are denoted following their common name). Of these, sufficient data are available only to evaluate Kava and St. John's Wort, with Kava demonstrating a significant treatment effect for anxiety (202), and St. John's Wort demonstrating effects on depressive symptoms superior to placebo and comparable to standard antidepressant treatments (207).

A practicing Canadian herbalist specializing in the treatment of FMS recommends groups of herbs targeting various aspects of FMS, including muscle stiffness and pain, a lack of energy, pain and lack of sleep, joint pain and inflammation, depression and digestive disturbance, and "brain fog" impaired memory. Over 37 herbs are recommended (211), many of which have also been cited in qualitative Appalachian herbal literature for the treatment of symptoms that overlap with FMS symptoms (FMS is not cited in historical Appalachian herbal literature since it is a relatively new diagnosis, although the related, older term "rheumatism" is used extensively) (Appendix B).

A qualitative study of rheumatism treatments used by a prominent Appalachian herbalist (first located in Southern Appalachia, followed by residency in West Virginia) identified twenty-three different plants used for rheumatism (212). Similar findings have been reported for various other illnesses, both in contemporary field studies and in older regional collections of herbal texts (213-215). A literature review of qualitative herbal literature (Appendix B) cites the anecdotal use of 101 herbs in the Appalachian region for pain relief $(121,179,180,216-219)$, rheumatism $(121,169,178-180,182,191,216-218)$, and arthritis $(121,169,178,179,191,212,217,218)$, while 12 were used for inflammatory issues $(121,179$, 180, 217, 218), 3 for alleviation of sleep troubles $(121,169,179)$, and 4 for headache $(179,180,217)$. It is not clear if these herbs continue to be used for these conditions in Appalachia.

Herbs that have been used historically in the Appalachian region, and for which there is some evidence of efficacy include: Willow (Salix sp.) for pain (220-223) and headache (224); Phytolodor herbal 
extract blend, which includes Ash bark (although a different Fraxinus species than that used in Appalachia) for musculoskeletal issues (123); Black Cohosh (Cimicifuga racemosa) and chamomile (Chamomilla recutita) for sleep (225-227); Hops (Humulus lupulus) for sleep $(223,228)$ and analgesia (223, 229, 230); Evening Primrose (Oenothera biennis) for rheumatoid arthritis (231); Ginger (Zingiber officinale ) for rheumatic pain and musculoskeletal disorders $(223,232,233)$; and Peppermint (Mentha piperita) for headache (223, 234, 235).

Herbs for which some evidence of efficacy for FMS symptoms or related conditions exist, but for which no usage in the Appalachian region has been cited, include Chili pepper ( $C$. frutecens and others), Cat's Claw (Uncaria tomentosa), Devil's Claw (Harpagophytum procubens), Feverfew (Tanacetum parthenium), St. John's Wort (Hypericum perforatum), and Valerian (Valeriana officinalis) (123). Despite the apparent promise of several herbs for relief of pain and related symptoms, there are few population-based studies, high-quality trials, systematic reviews, and meta-analyses regarding the use and efficacy of herbs that may benefit those with FMS and associated comorbidities. Therefore, further research on herbs as a potential treatment for FMS is needed, specifically in the Appalachian region where opioid use is epidemic.

\section{$\underline{\text { Relationship of Herbal Medicines, Inflammation, and Chronic Pain }}$}

A common underlying mechanism in the generation and promotion of chronic pain is the presence of inflammation at the site of the damaged or affected tissue (31); inflammatory responses in the peripheral and central nervous systems may also play key roles in the etiology of chronic pain (32). When the synthesis of prostaglandins is inhibited, inflammation may result, ultimately leading to pain. Chronic inflammation is characterized by a proliferation of fibroblasts and formation of blood vessels (angiogenesis), as well as an influx of chronic inflammatory cells, namely granulocytes (neutrophils, eosinophils, and basophils), lymphocytes, plasma cells and macrophages (236). When macrophages become activated, they produce cytokines and chemokines, some of which, in turn promote inflammation. C-reactive protein (CRP), part of an acute response to underlying infection or tissue injury (237-239), is now also believed to be a marker of chronic systemic inflammation (240). Because CRP levels have neither seasonal nor diurnal variations and are not influenced by food intake (237-239), CRP assay is commonly included as part of the diagnostic laboratory workup for many proinflammatory diseases, including FMS.

Herbs with evidence of anti-inflammatory action (in vitro and in vivo) which have historically been used in the Appalachian region (although exact rates of use are unknown) include Chamomile (Chamomilla recutita) (225-227), Dandelion (Taraxacum officinale) (223, 241-243), Common Elder (Sambucus canadensis) $(223,241,244,245)$, Eucalyptus (E. globulus) $(223,246)$, Hops (Humulus 
lupulus) (223, 243, 247), Mint (243), Watercress (243), Licorice (Glycyrrhiza glabra) (223, 248), Witch Hazel (Hamamelis virginiana) (223, 249), Skullcap (Scutellaria baicalensis) (250-252), Willow (Salix spp.) (223, 253, 254), and selected components of Skullcap (Scutellaria baicalensis) (255), Willow (Salix spp.) (256), Ginger (Zingiber officinale) (233, 257-261), Ginseng (Panax quinquefolia) (262, 263), and Horse Chestnut (Aesculus hippocastanum) (223, 264, 265). Leukocytes, which are associated with cytokine production in both immune response and inflammatory processes, are mobilized by the herb Echinacea (Echinacea spp.) as well (223, 266-268); moreover, in a 15-day experimental study in a female rat model of arthritis, CRP values decreased significantly in those administered Echinacea, compared to untreated animals (269). Oroxylin A, a natural flavonoid from Skullcap (Scutellaria baicalensis), significantly attenuated expression of inflammatory mediators (i.e., iNOS and COX-2) and cytokines (i.e., TNF-alpha, IL-1beta, and IL-6), in an estrogen receptor-dependent manner, suggesting anti-inflammatory effects (270).

Herbs for which some evidence of efficacy of anti-inflammatory action, but for which current prevalence rates in the Appalachian region have, to our knowledge, never been measured, include the ethanolic extract of Cat's Claw (Uncaria tomentosa) (123), Thunder God Vine (Tripterygium wilfordii) (123, 271), Stevia (243), Cinnamon (243), Turmeric (243), Mate (243), Boswellia (Boswellia serrata) (123), and Lavender (272), among others.

The relationship between many herbs and inflammatory markers may also be mediated in part by the elucidation of related antioxidant activity. Oxidative damage to membrane lipids, DNA, proteins, and lipoproteins, increases risk for several autoimmune and inflammatory diseases (273). Herbs are a rich source of polyphenol compounds (274) and their antioxidant activities are several times higher than those of vegetables (275) and fruits (276). Antioxidants contained in herbs may also suppress oxidative stress and related damage in the gastrointestinal tract before being absorbed (273), and thus, could modulate the level of oxidative stress by enhancing anti-inflammatory capacity $(277,278)$.

Because certain inflammatory cytokines are related to the development of contralateral hyperalgesia (an extreme, exaggerated pain response) and allodynia (central pain sensitization following painful, often repetitive stimulation) (33), which FMS patients experience (34), and because the modulation of inflammation and oxidative stress by herbs can be beneficial (277), the possible links between systemic inflammation and FMS, as well as herbal use and FMS, clearly warrant further investigation.

\section{Challenges in Herbal Medicine Research}

Methodological issues specific to research on herbal medicines include misclassification, ethnocentric, and recall biases. Areas in need of further exploration include the dialogue between physicians and patients regarding herbal use, safety, and efficacy; specifically, patient under-reporting of 
herbal use to health providers should be examined. Studies utilizing biobehavioral approaches may help bridge this gap, and aid in understanding/clarifying motivating factors in an individual's decision to use herbal medicine. Rigorous efficacy research on herbs remains sparse. Both rigorous population-based epidemiologic studies and high quality clinical trials are needed; moreover, also needed is the dissemination of evidence-based information that allows for clear interpretation regarding safety, efficacy, and limitations.

The most universal and basic definition of an herb is 'plant-derived material'. Herbs go by many names, often creating confusion among researchers and the public, and can lead experienced researchers to overlook research on plants of interest. Additionally, most available research is only available in the Chinese language (279), which may result in an ethnocentric bias in herbs considered safe and efficacious among differing populations.

Misclassification bias often exists, even in high-quality meta-analyses, due to the differences in constituents between the same herbs grown in different locations, prepared or processed under differing conditions, plant parts used, dose, and even plant variety. Therefore, the "pooling" of different products to reach conclusions about the efficacy of a single herb is often difficult, if not impossible (123).

Additional misclassification bias exists due to the "lumping" of other biologically-based therapies with herbs, such as vitamin or minerals, homeopathic supplements, fish oil supplements, and prebiotics/probiotics.

In many traditional medical systems, combination herbal formulae are more the rule than the exception. Many herbal practitioners individualize formulations and hold that combinations of plants may work together in a synergistic fashion, although this area has not been the subject of much scientific research (123). CHA users frequently use "bundles" of approaches rather than just one therapy in isolation (107), which can result in potential misclassification bias among CHA users who participate in poorly-designed observational studies, and clinical trials where exclusion criteria do not consider polypharmacy of herbal products and other CHAs.

A regrettable aspect of most folk medicine sources is that they provide scant information on dosage amount and intake regimen; dosing is often based on the number three or nine (times per day), and dosage directions may be vague (e.g., stipulating only "a potent" or "a right smart amount" of herb) (124). Recall bias in reporting specific herbs used may exist because of the more informal interactions which occur when herbalist practitioners meet with patients, compared to those which occur in clinical settings (182), where documenting procedures are more standardized. Additionally, preparation methods of herbs can vary greatly, and are typically not described in literature focused on herbal preparations that are not herbal supplements - and even for supplements, preparation and verification methods are not consistently described. 
Not all physicians inquire about CHA use among their patients (137). A UK systematic review indicated that, although patients believed CHAs to be effective, their doctors indicated skepticism about the usefulness of CHA in rheumatology $(192,194)$. Commonly, there is miscommunication between physicians and patients about CHA approaches for which potential safety concerns can arise. Patient care may be improved if this miscommunication is addressed. This communication gap arises from differences in cultures and belief systems: thus it requires a blend of intellectual flexibility and nonjudgmental openness as it enables the physician to weave the patient's "explanatory model", which includes CHA use as well as the application of their own understanding of geographically relevant folk illnesses [Figure 1]), into the fabric of care (108).

There is a clear need for the utilization of behavioral theories in measuring factors related to herbal use, as this can help to guide clinical practice. The reasoning behind current national trends in herbal use are unclear; trends may be due to factors other than individuals' willingness to follow traditional remedies passed down through families or communities, often with accompanying magicoreligious healing practices/folk healing. It is plausible that changes in environmental factors, communication within families and communities, and in the health care system overall may have contributed to an overall decline in the identification, preparation, and usage of traditional herbs in the U.S. and in the Appalachian region; there has been variation in herbal trends since the large, population studies of the 1990s $(90,106,125,280)$. Conversely, these or factors may have increased the usage of already-prepared herbs in supplement form, which are often readily available from multiple sources (e.g. Walmart) and promoted through various media (e.g. Dr. Oz television show, which attracts $\sim 3.5$ million daily television viewers)(281). Additionally, it is important to note "no individual ever completely replicates a single tradition" (108), which adds further complexity in understanding the reasoning for changes in herbal use trends. In our preliminary systematic review on the prevalence of herbal use among the general population in the US (Appendix C) ( $\mathrm{N}=32$ studies) $(109,116,118,137-165)$, only 7 studies $(\sim 22 \%)$ documented using social, behavioral, or economic theories/models as a guide $(116,145,150$, 152, 153, 156, 163). These theoretical approaches included the Anderson-Newman Behavioral Model of Health Services Utilization, the Socio-Behavioral Model of Healthcare Utilization, Grossman's model of Health Capital Investment, and Leventhal's Self-Regulatory Model. By using behavioral theories such as the Transtheoretical model and others, researchers and clinicians can begin to understand some of the drivers for changes in out-of-pocket healthcare costs associated with CHAs, and may alter the dialogue between patients and physicians to enable better treatment and ongoing, effective care for poorly managed chronic pain syndromes such as FMS.

Lastly, the proof required by participants in folk-medical tradition is very different from that demanded by medical researchers. For members of the former group, the efficacy of a home remedy, an herbal cure, or a magical procedure can be verified through anecdotal or experiential evidence alone (182). 
Some treatments are presumed to depend on the unique characteristics of the healer and on features of the healer-patient relationship (107). However, scientific research sometimes corroborates folk belief, as in the case of foxglove (Digitalis purpurea), which was prescribed as a heart tonic by community herbalists long before pharmacological studies confirmed its cardiotonic properties (182). There are many differences in the information flow pathways of efficacy research to the ultimate use of an herbal product versus a prescription (Rx) drug (Figure 3). The most obvious differences are: 1) Herbal use can occur even in the face of no or minimal scientific evidence. This differs from patient and clinician expectations regarding efficacy, and the ordered flow in prescription drug use, due to all constituents/ingredients known in the beginning of the Rx life cycle; 2) Herbal use often originates from anecdotal/qualitative literature. Therefore, the safety/efficacy is usually not known vs. a known safety/efficacy profile for Rx drugs; 3) Social media, social networks, and industry largely control the flow of information regarding evidence for herbal product use. Therefore, all information may be considered equally weighted evidence of efficacy among many herbal consumers. During a person's lifetime interaction with an herb, their perceived safety and efficacy of the herb may change depending on which source is recommending the herbal product. Although this may also occur with Rx drugs, it may be less likely due to more rigorous efficacy research requirements from the FDA and other regulatory bodies; 4) Doctors and pharmacists act as gatekeepers for Rx drug access, whereas there is no gatekeeper for herbal product access, since herbs are unregulated and potential users often have multiple avenues of access (i.e. pharmacies, health food stores, etc.). The current focus of herbal research is largely to identify constituents of herbs and their roles in various physiological pathways (282); thus, there may be a long waiting period before rigorous clinical research can verify the safety and efficacy of a wider variety of herbs, emphasizing the importance of conducting primary- and secondary-data analysis. When RCTs cannot be conducted, however, or when the results of RCTs may not be generalizable to the real world of CHA practice, it is necessary to use other study designs (107). Based on this, and the differences noted in the efficacy research information flow pathways of Rx drugs vs. herbs (Figure 3), epidemiological studies can play an important role for consumers of herbal products. For these reasons, researchers in this field must pay special attention to providing clear interpretations of their results for the public.

\section{Summary}

Complementary Health Approaches (CHAs) are frequently utilized to treat chronic pain syndromes, however, rigorous observational and clinical studies of herbal medicine are largely lacking. Additionally, no epidemiological studies have examined herbal use in Appalachian pain populations, where opioid misuse and abuse is epidemic. There is limited evidence suggesting those with Fibromyalgia Syndrome (FMS), a chronic pain syndrome with unknown etiology for which opioids are often prescribed, frequently use herbal medicine to help with FMS symptoms. Further, the Appalachian 
region, particularly West Virginia, has a rich history of anecdotal herbal medicine use for many symptoms overlapping with those of FMS. Understanding the correlates of herbal use in this population (including pain level as a potential motivator for herbal use) as well as patterns of herbal medicine among pain populations over time, will provide a window into patient health behaviors physicians may not be aware of. Importantly, these studies will also lay the foundation for efficacy studies examining interactions and synergistic effects of herbal medicine with opioids and other analgesic drugs. Further elucidation of the relationship between inflammation and FMS is also necessary due to the inconsistency of existing studies; understanding the potential role of inflammation in FMS is important since the presence of inflammation is intrinsically tied to chronic pain, and many herbs are used for their antiinflammatory and analgesic effects (Conceptual Model for this dissertation found in Figure 4).

Self-management of chronic pain is in the current translational focus of the National Institutes of Health (NIH); additionally, the NIH Pain Consortium has stated that the understanding and treatment of pain remains incomplete, thus, research with a theoretical underpinning is needed to determine the extent for which specific CHAs, including herbs, are used in pain populations, specifically, FMS. Further elucidation of the relationship between pain and inflammation is also essential. This research is critical, since pain is poorly handled by current treatment methods.

\section{Rationale}

1) Chronic pain management for FMS by conventional treatments is often inadequate, perhaps contributing to the elevated use of CHAs such as herbal medicine among chronic pain patients; 
2) While the use of herbs, like certain other CHAs, is likely high among those with chronic pain, little is known regarding herb use in specific chronic pain populations, including those who suffer from FMS; 3) Increased understanding of the patterns, correlates, and determinants of herbal and other CHA use is particularly important in chronic pain populations, given they are also likely to be on pharmacological treatments, including opioids, with the potential for interaction and/or abuse;

4) Appalachian adults, who reside in areas with some of the highest rates of opioid overdose deaths in the US, may be more likely to consume herbs for health benefit, given their rich traditions of herbal harvesting and consumption; and,

5) Increased understanding of herbal medicine and other CHA use in this population may aid in identifying new and potentially safer treatments for pain management, and help elucidate underlying mechanisms.

\section{Specific Aim 1}

Investigate if elevated blood serum levels of pro-inflammatory C-reactive protein (CRP) are associated with Fibromyalgia Syndrome (controlling for potential health factors, demographic variables, and lifestyle factors).

Objective 1.1. Determine the association between CRP and FMS diagnosis.

Hypothesis 1.1. Those with a FMS will have significantly elevated blood serum levels of CRP compared to those without FMS.

\section{Specific Aim 2}

To examine, in two nationally representative samples (2007 and 2012), potential associations between herbal supplement use and FMS diagnosis (controlling for potential health factors, demographic variables, and lifestyle factors) and potential changes in patterns of herbal use in this population over time.

Objective 2.1. Determine if those with a diagnosis of FMS were more likely to use herbal supplements than those without FMS in 2007 and 2012.

Hypotheses 2.1. Those with a FMS diagnosis will be more likely to use herbal supplements than those without fibromyalgia syndrome in both 2007 and 2012.

Objective 2.2. Determine if the prevalence of herbal supplement use among those with FMS diagnosis changed between 2007 and 2012. 
Hypothesis 2.2. Prevalence rates for herbal supplement use will increase over time among those with FMS.

\section{Specific Aim 3}

Using a newly developed pain survey instrument, explore the patterns and correlates of herbal medicine use in a sample of WV pain patients, including potential variation by pain status.

Objective 3.1. Assess potential correlates of herbal medicine and other CHAs used in northern West Virginia (WV) pain patients, through the use of our recently established and pilot-tested pain survey utilizing a theoretically-guided novel $\mathrm{CHA} /$ herbal medicine measurement tool

Hypothesis 3.1. Herbal medicine used for pain in Northern WV will be significantly associated with a variety of demographic, lifestyle, and health factors, including the use of other complementary health approaches and many health conditions.

Objective 3.2. Provide a list of patient-reported herbal identification, with accompanying frequencies

Hypothesis 3.2. Northern WV pain patients will report a variety of herbs used for pain management

The three specific aims/studies listed above correspond to chapters two, three, and four. Chapter 5 presents a summary of key findings of these studies, strengths, limitations, potential public health implications as well as potential direction and recommendations for future research.

To our knowledge, no research has investigated inflammation as a potential mediator between herbal use and chronic pain, nor characterized varying levels of pain as a motivator for herbal medicine use. Additionally, no research (to our knowledge) has attempted to quantify the herbs potentially used in Appalachian folk medicine for pain

The Institute of Medicine stresses the importance of increased research into the translation of effective treatments into practice and into the occurrence and cost of pain (6). Each aim of this dissertation will use cross-sectional data to enhance our understanding of the prevalence, patterns, and correlates of the herbal medicine use among those with chronic pain. This information will help inform practitioners working with chronic pain populations, will lay the groundwork for additional exploration regarding the role of inflammation in chronic pain, identify new and potentially safer treatments for pain management, and may form the foundation for larger survey studies, future prospective studies, and clinical trials. 


\section{CHAPTER 2: The association of Fibromyalgia to serum C-reactive protein in a large Appalachian population}

\section{Introduction}

Chronic pain, defined as ongoing or recurrent pain that extends beyond the usual course of acute illness or injury for at least three to six months, is debilitating and costly. Over $20 \%$ of adults experience chronic pain at some point in their lives (3). Fibromyalgia syndrome (FMS) is a rheumatologic chronic pain syndrome affecting approximately $0.5-5 \%$ of populations in developed countries (7), including $1.75 \%$ of those in the U.S. (8). FMS is characterized by a constellation of somatic symptoms that are typically present in addition to widespread pain (e.g., fatigue, sleep disturbance, memory, and mood problems), for which no clear cause can be found (9). FMS is typically accompanied by morning stiffness, and sensitivity to loud noises, bright lights, and temperature extremes; women with FMS often report painful menstrual periods (13). Although FMS affects both sexes and people of all ages, the majority (80-90\%) have been Caucasian $(13,14)$ middle-aged women (13). Family members of FMS patients are at a higher risk for FMS (15); the cause is unknown.

While the etiology of FMS remains poorly understood, the widespread pain of FMS is thought to reflect abnormal central nervous system sensory information processing, with altered function in pain pathways and neuroendocrine disturbance (9); Inflammatory processes may also play a significant role in the pathogenesis of FMS (283). Although several MicroRNAs have been associated with FMS and symptom severity (19), diagnosis of FMS remains challenging, and there are currently no definitive diagnostic laboratory tests for the disease (18).

C-reactive protein (CRP), previously considered a biomarker of underlying infection or tissue injury (237), is now also believed to reflect chronic systemic inflammation (240). CRP is considered a reliable proinflammatory biomarker (237) and is often included as part of the diagnostic laboratory workup for many rheumatological conditions (237). While CRP is often included in the diagnostic workup for FMS, the relationship of CRP to FMS has not been clearly established.

Inflammation, as a characteristic reaction of tissues to injury or disease marked by physical swelling, redness, heat, and pain upon clinical examination, is not a classical symptom of FMS (18). Inflammatory cytokines promote the development of contralateral hyperalgesia (an extreme, exaggerated reaction to pain) and allodynia (central pain sensitization following painful, often repetitive stimulation) (33). FMS is typically characterized by both (34), and most (35-49), but not all recent studies $(43,50)$ have suggested a possible link between systemic inflammation and FMS. Of these, five studies measured CRP (44, 48, 49, 51, 284); four suggested a positive association between CRP and FMS (44, 48, 49, 284). 
However, to date, only one large, cross-sectional, population-based study has examined the association of CRP to FMS (48). The study excluded women, and combined FMS with other pain syndromes, rendering assessment of the specific relationship between CRP and FMS difficult.

Moreover, to our knowledge, no studies to date examining the association of CRP to FMS have investigated the potential mediating effects of sleep or mood disturbance, factors linked to both elevated CRP levels and FMS (285-288). Few have assessed the influence of elevated BMI (287, 289), comorbidities, and other correlates (290). This large, population-based study will fill the gaps in our understanding of the potential influence(s) of mood, sleep, BMI, and comorbid conditions on the relationship between CRP and FMS.

In this study, we investigated the relation of serum CRP levels to FMS in a large population of Appalachian adults. We hypothesized that CRP serum levels would be significantly and positively associated with diagnosed FMS after adjustment for potential confounding factors.

\section{Methods}

\section{Data Source}

This cross-sectional study used data from the C8 Health Project, which was conducted as part of the settlement of a class-action lawsuit stemming from drinking water contamination by Perfluorooctanoic Acid (PFOA) released from the DuPont Washington Works Plant near Parkersburg, WV, USA (291). Data collection was conducted in 2005-2006 on individuals living or working in 6 PFOA-contaminated public water districts in West Virginia (WV) and Ohio (including those exposed to contaminated private-well drinking water) since 1951; a total of 69,030 individuals participated in the study, including $81 \%$ of eligible adults (291). Project data collection was administered by an independent company, Brookmar, Inc. (Parkersburg, WV), and was conducted under the authority and supervision of the Wood County, WV, Circuit Court. Participants completed a comprehensive health questionnaire and volunteered a blood sample after completing individual consent forms for both. Demographic data and health survey completion were verified by trained project staff (291). Project procedures, blood processing and assay methods, along with quality-assurance measures, have been described in detail elsewhere (291). Briefly, following collection of each blood sample, serum was separated from red blood cells into single-use aliquots by centrifusion, and was refrigerated at individual data collection sites until daily pickup from a large, independent, accredited clinical diagnostic laboratory (LabCorp, Inc., Burlington, NC, USA). Samples were transported to a regional processing center (LabCorp, Inc., 
Columbus, $\mathrm{OH}$ ) where they underwent analysis by latex immunoturbidimetry on a COBAS Integra 800 (Roche, Germany). The West Virginia University (WVU) IRB permitted access to the de-identified data by WVU investigators. Demographic, lifestyle, and health characteristics were determined via self-report; diagnoses of certain disorders, including diabetes and cardiovascular diseases, were further verified via chart review.

\section{Study Population}

Our analysis excluded participants who were missing data on age or $<18$ years of age $(n=12471$, $18.1 \%)$, pregnant $(n=640,1.1 \%)$; those reporting a cancer diagnosis and receiving treatment for diagnosed cancer other than non-melanoma skin cancer $(n=437,0.77 \%)$ to eliminate potential bias introduced by varied CRP levels as a result of chemotherapy treatment; those with service-related disabilities $(n=710,1.3 \%)$; and those who did not complete both the survey and blood work $(n=468$, $0.83 \%)$. Participants with extreme body mass index (BMI) values $(<10.5$ and $>60.0)$ were also excluded to eliminate potential information bias $(n=94,0.17 \%)$. Further exclusion of persons with missing data on CRP and FMS $(n=40,0.002 \%)$ and other covariates of interest $(n=1673,3.1 \%)$, with the exception of covariates for which missing data on $>10 \%$ of participants occurred (for which a 'missing' category was included in analysis), yielded a final study sample of 52,535, including 51,410 FMS-free controls and 1,125 adults with FMS (Figure 1).

\section{Variables}

Outcome Variable. Our primary, dichotomous outcome variable was FMS diagnosis, which was ascertained via self-report response to the question "Have you ever been diagnosed with Fibromyalgia?"

Exposure Variable. Our primary exposure variable was serum level of inflammatory marker CRP (mg/L). C8 Health Project coordinators recoded CRP values below the level of detection to 50\% of the lowest level (i.e., “ $<0.3$ ” $\rightarrow 0.15$ ). Likewise, values above the level of detection were recoded to $50 \%$ above the maximum level.

Covariates. In addition to age, gender, race, education, employment, marital status, income, alcohol or tobacco use, and exercise program status, comorbid conditions were also selected a priori as covariates if known or suspected to be associated with FMS and/or CRP. These included BMI (21), autoimmune conditions (22), osteoarthritis (23), kidney (24), respiratory (25), cardiovascular (24), liver (26), and endocrine (24) disease, diabetes (27), severe allergies (24) and sinus disease (28), stomach conditions (24, 26), and headache (29). PFOA serum level and reproductive factors (292) were also examined as covariates. 
Potential mediating and modifying factors. Potential mediating and modifying factors included fatigue, sleep impairment (26), mood disturbance (24, 26, 286), age (13), gender (13), obesity (289), tobacco use (293), statin use (294), and reproductive factors (292).

\section{Statistical Analysis}

We conducted complete-case analyses using SAS 9.4 (Cary, NC, USA). Logistic regression analyses were used to evaluate the independent association of CRP level to FMS status, and to assess the influence of potential confounding, mediating, and modifying factors. The primary explanatory variable of interest, CRP, was analyzed as a continuous and categorical variable (study population quartiles, with the lowest percentile group used as the referent). Linear trends for CRP quartiles were assessed using polynomial contrasts. Differences between participants missing any data were assessed using logistic regression. Cox-Snell $\mathrm{R}^{2}$ values measured the predictive power of models. All p-values shown are twosided.

All demographic characteristics, in addition to lifestyle factors significantly differing by FMS, were controlled for in multivariate models. BMI was categorized using the National Institutes of Health clinical classifications (scores of $<25=$ 'Underweight or Normal weight'; 25 - 29.9='Overweight'; 30 34.9='Obese Class 1'; and, 35+='Obese Classes 2/3') (295).

We evaluated the influence of both specific comorbid conditions and total number of comorbid conditions on the relation of CRP to FMS. A comorbidity index was created based on number of chronic comorbid conditions reported; these included: autoimmune disorders (defined as having any diagnoses of immune disease, lupus, or rheumatoid arthritis), osteoarthritis, allergies or frequent sinusitis, kidney disease, respiratory conditions (asthma, emphysema, chronic obstructive pulmonary disorder, bronchitis), heart disease, liver disease, endocrine disorders (thyroid, Addison's, or Cushing's disease), diabetes, or frequent headaches. This index was evaluated as both a continuous and categorical variable ('none', '1 comorbidity', 2 comorbidities', and ' $3+$ comorbidities').

We assessed potential mediating influences of sleep impairment and mood disturbance. A composite sleep quality variable, with higher scores indicating poorer sleep quality, was derived from responses to individual questions regarding the frequency of short sleep, fitful sleep, insomnia, and/or daytime somnolence (scored as follows: 3='frequently', 2='sometimes', 1='rarely', and 0 ='never', for each). Mood disturbance was also assessed as a composite variable from responses to four individual questions, with higher scores indicating frequent mood swings, irritability, fatigue, and/or inability to concentrate (where 3='frequently', 2='sometimes', 1='rarely', and 0 ='never').

Additionally, we assessed the potential modifying influence of age ( $<45 \mathrm{vs.} \geq 45$ years), tobacco use ('current'/'not currently using tobacco'), gender, obesity (BMI $<30$ vs. $\geq 30$ ) current menstruation 
(yes/no), menopausal status (peri- or post-menopausal/premenopausal), statin use (yes/no), and sleep impairment and mood disturbance (scores of $<6$ vs. $\geq 6$, respectively) on the association between CRP and FMS.

We also conducted separate ancillary analyses adjusting for statin use (yes/no), PFOA serum level (ng/mL, evaluated as quartiles), and reproductive characteristics (women only) to determine the potential confounding influence of these factors on any observed association between CRP and FMS. Reproductive factors assessed included menopausal status ('pre-menopausal,' 'peri-or post-menopausal,' or 'unsure'), parity (number of pregnancies), and age at menarche (' $16+$ years of age'/‘other age or unsure').

The first regression model assessed the crude association of CRP levels to reported FMS diagnosis. The second model was adjusted for demographic and lifestyle covariates, while additional models adjusted for BMI and comorbid conditions, as well as sleep and mood disturbance (separately and combined, respectively). We conducted additional analyses to evaluate the potential confounding influence of statin use, PFOA serum level, and (in female participants) reproductive history (i.e., menopausal status, age at menarche, and parity).

The potential mediating effects of BMI and comorbid conditions, as well as sleep impairment and mood disturbance were examined using separate logistic regression models. We also conducted ancillary analyses excluding autoimmune disorders. To evaluate the potential modifying effects of age, smoking, gender, obesity, current menstruation, menopausal status, statin use, sleep impairment, and mood disturbance, we conducted multivariable analyses; interactions were assessed by including the corresponding multiplicative-interaction term in the statistical models.

\section{Results}

Relative to participants with complete data, those with missing data on key covariates had a higher number of comorbidities and were more likely to be poorly educated or to be retired, disabled, or unemployed ( $p$ 's $\leq .002$ ), but did not differ in other factors. Demographic and lifestyle characteristics are displayed in Table 1. Study participants were predominantly white (97.2\%), female (52.4\%), and married or cohabitating (68.9\%). Participant age ranged from 18.0 to 105.2 years, averaging $45.3(\mathrm{SD}=16.1)$ years. Most participants were employed or students (64.1\%) and overweight $(69.0 \% \mathrm{BMI} \geq 25$, mean BMI $=28.5, \mathrm{SD}=6.3)$, while nearly half did not currently consume alcohol $(51.1 \%)$ and never smoked (43.5\%). CRP serum levels varied from 0.15 to 250.6 (mean CRP level $=3.79, \mathrm{SD}=7.2$ ) $\mathrm{mg} / \mathrm{L}$.

FMS was present in $2.1 \%$ of the study population $(n=1125)$. After adjustment for demographic and lifestyle factors, the odds of FMS increased $2 \%$ for every year-unit increase in age (Adjusted odds 
ratio $(\mathrm{AOR})=1.02,95 \%$ confidence interval $(\mathrm{CI}): 1.02,1.03$ (Table 1$)$ ), with a significantly higher mean age among FMS cases $(M=51.1, \mathrm{SD}=11.6)$ compared to controls $(M=45.1, \mathrm{SD}=16.2)$. Those aged 45-64 years were 8.3 times more likely $(\mathrm{AOR}=8.28, \mathrm{CI} 4.91,13.95)$ than those in the age group 18-24 years to have FMS. Those with FMS were nearly 11 times more likely to be female (AOR $=10.68, \mathrm{CI}$ $8.53,13.37)$. Participants who were employed or students were less likely to have FMS than all others, as were those with $<12^{\text {th }}$ grade education, compared to those with higher educational attainment. Relative to participants who were married or cohabitating, those who were single were almost $40 \%$ less likely to report a diagnosis of FMS (AOR $=0.63$, CI 0.47, 0.84). Participants who reported current consumption of alcohol were slightly less likely to report a diagnosis of FMS $(35.8 \%$ vs. $49.2 \%$, respectively; AOR = 0.86 , CI $0.75,0.98$ ), and women who had experienced menopause were about $80 \%$ more likely to have an FMS diagnosis compared to pre-menopausal women (AOR = 1.82, CI 1.49, 2.21).

Health characteristics of the study population are detailed in Table 2. Mean BMI was significantly higher among FMS cases $(M=30.47, \mathrm{SD}=7.2)$ than controls $(M=28.49, \mathrm{SD}=6.3)$, and was significantly and positively associated with FMS after adjustment for demographic and lifestyle factors (AOR per unit BMI increase $=1.02$, CI 1.01, 1.03). Relative to those with a BMI <25, participants who were obese were significantly more likely to have FMS ( $\mathrm{AOR}=1.24, \mathrm{CI} 1.02,1.49$; and, $\mathrm{AOR}=$ 1.43, CI 1.18, 1.74, for BMI 30-35 and BMI >35, respectively). There was a $60 \%$ increase in FMS diagnosis for each additional comorbidity $(\mathrm{AOR}=1.60$, CI 1.54, 1.67 (Table 2); participants reporting a diagnosis of 3 or more comorbidities were 10.5 times more likely to have FMS (AOR = 10.46, CI 7.90, 13.84) compared to those with no comorbidities. FMS was likewise strongly and positively associated with most chronic conditions evaluated, including in order of decreasing magnitude osteoarthritis $(\mathrm{AOR}=$ 3.86, CI 3.34, 4.45), autoimmune disease $(\mathrm{AOR}=3.43$, CI 2.89, 4.06), allergies/chronic sinusitis ( $\mathrm{AOR}=$ 2.91, CI 2.51, 3.38), frequent headaches ( $\mathrm{AOR}=2.03$, CI 1.78, 2.31), and kidney ( $\mathrm{AOR}=1.72$, CI 1.47, $2.01)$, respiratory $(\mathrm{AOR}=1.89, \mathrm{CI} 1.64,2.17)$, endocrine $(\mathrm{AOR}=1.82, \mathrm{CI} 1.57,2.12)$, and liver $(\mathrm{AOR}=$ 1.51, CI 1.06, 2.16) diseases.

FMS cases reported higher sleep $(M=7.3, \mathrm{SD}=3.3$ vs. $M=3.9, \mathrm{SD}=3.5)$ and mood disturbance scores $(M=7.3, \mathrm{SD}=3.4$ vs. $M=4.5, \mathrm{SD}=3.7)$ than did controls (Table 2$)$. After adjustment for demographic and lifestyle factors, FMS remained strongly and positively related to scores for both sleep $(\mathrm{AOR}=1.23, \mathrm{CI} 1.21,1.25)$, and mood disturbance $(\mathrm{AOR}=1.19, \mathrm{CI} 1.17,1.21)$.

Relation of CRP to FMS. Mean CRP (mg/L) was significantly higher among FMS cases $(M=5.54$, SD = 9.8) compared to controls $(M=3.75, \mathrm{SD}=7.2)$ (Table 3 ). CRP serum level showed a positive association with FMS (unadjusted OR for highest vs. lowest quartile = 2.50, CI 2.10, 2.97; P for trend $<.0001$ ); adjustment for demographic and lifestyle factors substantially attenuated but did not eliminate this association (AOR for highest vs. lowest quartile $=1.35$, CI 1.13, 1.62; P for trend <.0001). Analysis of CRP as a continuous variable yielded similar findings, with odds of FMS increasing by $2 \%$ for each 
one $\mathrm{mg} / \mathrm{L}$ of CRP rise (unadjusted $\mathrm{OR}=1.02$, CI 1.01, 1.02); adjustment for demographic and lifestyle factors slightly attenuated this association $(\mathrm{AOR}=1.01$, CI 1.00, 1.01).

The addition of BMI and comorbidities to the model further weakened the relationship between CRP and FMS (AORs for highest vs. lowest CRP quartile = 1.17 (CI 0.96, 1.42) and 1.10 (CI 0.92, 1.32), for BMI and comorbidities, respectively; and, combined $\mathrm{OR}=1.07$ (CI 0.88, 1.30)) suggesting that these factors may at least partially explain the observed associations (Table 3). The inclusion of mood disturbance and sleep impairment, separately and combined, only slightly attenuated the association of FMS to CRP after adjustment for demographic and lifestyle factors (AOR = 1.01, CI 1.00, 1.01) (Table 4). These findings suggest that any mediating effect of these factors was modest.

Additional adjustment for statin use, PFOA, and female reproductive characteristics (menopausal status, age at menarche, and parity) did not appreciably change the relationship between CRP and FMS. Similarly, exclusion of those with rheumatoid arthritis and other autoimmune conditions did not appreciably affect risk estimates. Likewise, we found no evidence for a modifying effect of age, tobacco use, gender, obesity, menopausal status, or other factors on the relationship between CRP and FMS.

\section{Discussion}

This is the first large, population-based investigation to examine the relationship between CRP and FMS, to assess the potential influence of BMI and comorbid conditions on this relationship, and to evaluate the potential mediating role of mood and sleep impairment. In this cross-sectional study of a large Appalachian population, CRP serum level showed a positive association with FMS, which remained significant after adjustment for multiple demographic and lifestyle factors, including age, gender, education level, employment, marital status, alcohol, and tobacco use. Adjustment for BMI and comorbid conditions substantially attenuated this relationship. These findings are broadly consistent with those from a recent cross-sectional investigation of 5110 Norwegian men; cases with FMS/Chronic Pain Syndrome showed a strong, positive relationship to high sensitivity-CRP level (OR for $\geq 10 \mathrm{mg} / \mathrm{L}$ vs. $<1$ $\mathrm{mg} / \mathrm{L}=2.6,95 \% \mathrm{CI}: 1.4,4.6, p=.002 ; \mathrm{P}$ for trend $=.006)$ after adjustment for age, education, smoking, and cholesterol medication (48). In contrast, as stated in the introduction, findings from smaller casecontrol studies examining the association between CRP and FMS have been inconsistent (44, 46, 49, 51), perhaps due to varying sample sizes and differing selection criteria among controls; of the four casecontrol studies published to date, only one reported significantly higher CRP levels in FMS patients compared to healthy controls after adjustment for age, sex, and race (44).

As indicated above, the inclusion of BMI in our model substantially attenuated the association of FMS to CRP, suggesting BMI may have largely explain the elevated CRP levels in FMS; this was second 
to the inclusion of comorbidities. Only one study to date has considered the potential contribution of BMI to the profile of CRP in FMS, with a strong and positive correlation (44). Ours is the first study to assess the contribution of multiple comorbidities on the relation of CRP and FMS. Additionally, in agreement with our study, others have suggested a mediating effect of BMI on the relation between CRP and FMS; FMS symptom improvement has followed weight loss among several FMS cases (296), and a longitudinal study revealed regular exercise and maintenance of body weight lowered the risk of FMS (297). Additionally, one case-control study found that after adjustment for demographic factors, BMI was the only significant contributor in a model exploring the relation between CRP and FMS $(\mathrm{r}=.062, p<$ 0.001) (44).

Comorbidities characterized by pain may contribute to the development of FMS; shared pain mechanisms between FMS and other conditions with a similar underlying pathophysiology (e.g., tension headache) or as a comorbidity characterized by inflammation or ongoing peripheral damage (e.g., autoimmune disorders and osteoarthritis) and FMS have recently been explored (9, 23, 298). Our study found that as the number of comorbidities increased, the odds of FMS increased as well.

Additionally, the presence of sleep impairment and mood disturbance modestly attenuated, but did not eliminate, the association between CRP and FMS. No existing studies have examined the role of sleep impairment on this relationship. We found no evidence supporting mood disorder as an effect modifier, similar to a study finding where CRP level did not differ by psychiatric status among those with FMS (47). The influence of sleep impairment and mood disturbance on the relation between CRP and FMS in our study likely reflects a bidirectional relationship.

The prevalence rate of FMS in our study (2.1\%) mirrored recent estimates for the U.S. population $(8,299)$. Consistent with previous studies, we found the likelihood of FMS diagnosis was increased in middle-aged females $(8,13)$. However, in contrast to findings from two large studies $(8,300)$, those who were married or reported higher levels of education were more likely to report FMS. Females who had experienced menopause were 1.8 times more likely to have FMS than those who had not; however, a lack of epidemiological research exists in the topical area of sex hormones, neurotransmitters, and FMS. We observed no significant association between age at menarche or parity and FMS, unlike a German casecontrol study of 653 middle-aged women (FMS cases = 36) which found, after controlling for age, those with FMS had significantly later menarche and were less likely to have ever been pregnant (301).

In agreement with previous cross-sectional and longitudinal studies (8, 297, 302), BMI was strongly and positively related to reported FMS in this large Appalachian population. Additionally, higher BMI has been associated with an increased risk of FMS after adjustment for mood and/or other health factors $(8,297)$, including familial FMS diagnosis (302). Likewise, consistent with findings from a recent cross-sectional study of a nationally representative sample of U.S. adults $(\mathrm{N}=8446$, FMS cases $=201)$

(8) FMS showed significant positive associations to multiple comorbid conditions in our study after 
adjustment for demographic and lifestyle factors; these included cardiovascular disease, rheumatoid arthritis and other autoimmune disorders, kidney, respiratory and liver diseases, and frequent headache. Osteoarthritis was also associated with a nearly 4-fold likelihood of FMS, dissimilar from a large, national analysis of U.S. electronic medical records $(\mathrm{N}=587961$, FMS cases $=4296)$ of only a (unadjusted) 2-fold likelihood (303). In contrast to other large studies of U.S. adults (8, 303), diabetes was not associated with FMS in this sample of Appalachian adults.

The strengths of this study were its high response rate, population-based design, and large sample size; this was the largest comprehensive community study conducted to date in the Appalachian region. We were able to control for a large number of potential confounders, including comorbid conditions. Misclassification of CRP was unlikely due to standardized assay procedures used.

This study targeted a population of predominantly white Appalachian adults, potentially limiting generalizability. Possible misclassification of FMS may have occurred depending on time the participant was diagnosed and the physician's awareness and use of the American College of Rheumatology classification criteria for FMS first established in 1990. In particular, poor or incomplete recognition of FMS by healthcare providers may have led to under-ascertainment (304). However, such underascertainment would be expected to bias the observed associations toward the null. FMS, in addition to most other assessed health conditions, was based on self-report, possibly leading to response or misclassification bias. To our knowledge, no clinical validation study has assessed the agreement between self-report and medical record-verified data on FMS.

Unmeasured confounding may have also contributed to our findings, although our ability to control for a large number of both known and potential risk factors for FMS diminishes this possibility. Our study also lacked specific information on certain conditions previously linked to FMS, including sleep apnea and irritable bowel syndrome. Although contact with former residents of counties used for study inclusion was attempted, some may not have participated in the study, possibly introducing sampling bias. Lastly, we cannot draw any conclusions regarding causality due to the lack of a temporal component in our cross-sectional study design.

\section{Conclusion}

In this large cross-sectional study of Appalachian adults we observed a significant, positive association between serum CRP and diagnosed FMS, which was largely explained by elevated BMI and chronic comorbid conditions. Adjustment for sleep and mood disturbance only modestly attenuated this association, suggesting that BMI and chronic comorbid conditions may largely account for elevated CRP levels among FMS patients over the presence of sleep and mood disturbance. Further prospective 
research is needed to determine the relation of CRP and other inflammatory markers to the development and progression of FMS, and to clarify the potentially complex role of obesity and comorbidities, in the potentially causal pathway between inflammatory markers and FMS. 


\section{CHAPTER 3: Prevalence Trends and the Association between Herbal Supplement Use and Fibromyalgia Syndrome in two National Samples (2007-2012)}

\section{Introduction}

Fibromyalgia syndrome (FMS) is a rheumatologic chronic pain syndrome affecting approximately $0.5-5 \%$ of populations in developed countries (7), including $1.75 \%$ of those in the U.S.(8). FMS is characterized by a constellation of somatic symptoms that are typically present in addition to widespread pain (e.g., fatigue, sleep disturbance, memory, and mood problems), for which no clear cause can be found (9). Additionally, those with FMS also experience high rates of comorbidity, such as autoimmune disease (22), osteoarthritis (23), kidney (24), respiratory (25), cardiovascular (24), liver (26), diabetes (27), stomach conditions $(24,26)$, and headache (29). Although FMS affects both sexes and people of all ages, the majority (80-90\%) of cases occur in Caucasian $(13,14)$ middle-aged women (13).

Chronic pain, such as that accompanying FMS, is difficult to manage; this is partially due to limited efficacy of conventional pharmaceutical therapies and the significant side effects associated with these medications. In addition, although specific clinical criteria for FMS, first developed by the American College of Rheumatology (ACR) in 1990, have been in effect for over 25 years, many physicians remain unfamiliar with FMS and FMS treatment (305). Therefore, many FMS patients have experienced difficulty in receiving diagnosis and consistent care for their symptoms, particularly before less constrictive ACR criteria were introduced in 2010 (306), which additionally gauges impact of FMS upon quality of life through the use of an impact score (17).

Although there is no evidence of efficacy for opioids as treatment for FMS $(18,75)$, more than $80 \%$ of FMS patients receive opioid therapy (63). Some physicians are reluctant to prescribe opioids (6), while others overprescribe them (85), even though prescription drug overdose deaths involving opioids (using age-adjusted data) have more than quadrupled since 1999 (84). People with FMS are also typically treated with antidepressants (54), muscle relaxants (11), as well as other pain (54) and sleep medicines (11). Drugs commonly used off-label to treat FMS include Gabapentin (56), Fluoxetine (57), Pramipexole (57), Amitriptyline (54), and Tramadol plus Paracetamol (57), among others (307); all carry significant side effects. Although three drugs (Anticonvulsant Lyrica/Pregabalin, and Serotonin-norepinephrine reuptake inhibitors Cymbalta/Duloxetine $(58,59)$ and Savella/Milnacipran) have been approved by the FDA for FMS treatment, they also carry significant side effects $(57,60,61)$, and the mechanisms by which these drugs alleviate FMS symptoms is unknown. For some FMS patients, these drugs are not effective (11). Chronic Nonsteroidal Anti-Inflammatory Drugs (NSAIDs) are also given as treatment for 
some pain symptoms (18), but likewise carry significant side effects for as many as $25 \%$ of long-term users (62).

Complementary Health Approaches (CHAs) (formerly referred to as 'Complementary and Alternative Medicine', or 'CAM' by the National Institute for Complementary and Integrative Medicine) are medical and health care systems, practices, and are often used by those with chronic pain(308). The ACR suggests people with FMS consider non-drug treatments in addition to their pharmaceutical approaches, and that certain CHAs may be helpful in managing FMS symptoms. Many of these treatments, including herbal dietary supplements, have not been well tested in FMS patients for safety and efficacy (76-78). Although CHA use is popular in the U.S., patients often do not disclose their use of CHAs to their physicians $(97,98)$, largely because the role of CHA in chronic pain management remains controversial. Physicians disagree with one another and with their patients about the cost, validity, and effectiveness of individual CHAs (99). The use of CHA use may reflect greater autonomy patient populations who are less dependent upon conventional medicine and wish to direct their own health care (88); some patients also use CHAs due to concerns over the safety of conventional medicine (100). Compared with nonusers, CHA users tend to be women, white, middle-aged, of higher income, and better-educated (90, 106, 110-116).

In the United States, 30-60\% of the general population use CHAs (88), and in 2007, about $\$ 14.8$ billion was spent on the purchase of non-vitamin, non-mineral, natural CHA products (109). According to the 2012 National Health Interview Survey (NHIS), which included a comprehensive survey on the use of CHAs in the U.S., $17.7 \%$ of adults had used a dietary supplement other than vitamins and minerals in the past year, making these products the most commonly used CHA for which data were available (104); these supplements included herbal products (Image 1) Consistent with these findings, systematic reviews of population-based studies in U.S. adults (2001-2012) indicate $18.1 \%$ of the general population used herbal medicine (109, 116, 118, 137-165). Echinacea, Ginseng, Ginkgo, and Garlic were the most common natural products among adults in 2007 (161).

'Herbs' (plant-derived products also known as 'botanicals' or 'biologically based therapies') are often sold as dietary supplements and are part of the broader CHA group of approaches called 'Natural Products', which also include (non plant-derived) vitamins and minerals. The use of herbs for potential health benefit is called 'herbal medicine use' or 'phytomedicine' and is part of many 'folk medicine' traditions. Many herbs have been documented to have anti-inflammatory action (in vitro and in vivo) $(123,223,225-227,233,241-265,271)$, and anecdotal accounts demonstrate herbalists have recommended many herbs for the treatment of FMS and/or related symptoms such as pain $(121,179,180$, 211, 216-219), rheumatism $(121,169,178-180,182,191,216-218)$, and arthritis $(121,169,178,179$, 
191, 212, 217, 218); others have been used for non-specific inflammation (121, 179, 180, 217, 218), sleep troubles $(121,169,179)$, and for headache $(179,180,217)$; it is not clear if these herbs continue to be used by those with FMS. A 2009 case-control study (FMS = 434) showed nearly half $(42.9 \%)$ of those with FMS used herbs and other supplements; herbs used most often by those with FMS were Ginkgo (3.7\%), Garlic (3.0\%), Ginseng (3.0\%), Echinacea (2.5\%), Evening Primrose oil (2.3\%), and Valerian (2.3\%) (197).

Pain-related disorders are the most commonly reported conditions for which patients use CHAs $(86,88,93,105,106)$. Those in the US most frequently use CHAs for back pain, neck pain, joint pain, and arthritis (161). However, investigations regarding the prevalence and correlates of herbal use in chronic pain populations remains limited, and even fewer studies have examined the relation of FMS to herbal use; one case-control study assessing this relationship combined herbal supplements with other natural products (197). Further, only one large study examined herbal use in a pain population receiving opioids (93), while UK population studies conducted over three decades ago examining similar chronic pain syndromes to FMS used small sample sizes $(\mathrm{N}<100)$.

Further research on FMS and herbal use is clearly warranted given that FMS is poorly managed by current treatment options, and there is a national opioid epidemic; while previous studies suggest CHA use is popular, research regarding herbal use for pain remains limited, particularly before and after implementation of updated FMS diagnostic criteria. We hypothesize that rates of herbal supplement use will differ between time points among those with FMS, and those with FMS will be more likely to use herbal supplements than those without FMS.

\section{Methods}

\section{Data Sources}

In two nationally nationally-representative samples of 23,501 and 34,525 U.S. adults (the National Health Interview Surveys (NHIS), 2007 and 2012, respectively), we investigated if those with Fibromyalgia Syndrome (FMS) were more likely to use herbal supplements than those without FMS, and determined if prevalence rates for herbal supplement use among those with FMS significantly changed over a 5-year time period. The NHIS is an annual national, cross-sectional household survey of the noninstitutionalized US population and is administered by the Centers for Disease Control and Prevention's (CDC) National Center for Health Statistics. An additional supplement (Adult Alternative Medicine) to the core individual, family, and household surveys in both years asked participants about use of a broad range of CHAs, including herbal supplements. All questions were administered in a 
personal interview format. Adult response rates to the NHIS were $78.3 \%$ in 2007 and $79.7 \%$ in 2012, respectively $(309,310)$.

The NHIS is the only national, public-use survey that includes comprehensive sets of questions regarding herbal and other CHA use. Both 2007 and 2012 surveys used a stratified multistage probability design weighted on age, sex, race/ethnicity; using 2000 and 2010 Census data for 2007 and 2012, respectively. Both surveys oversampled Asian, Black, and Hispanic persons, and the minority elderly population. Thus, each person in the covered population had a known non-zero probability of selection. These probabilities of selection, along with adjustments for nonresponse and post-stratification (defined as post-survey implementation stratification of the sample according to auxiliary information about the sampled population, similar to direct standardization) were reflected in the sample weights and complex survey procedures recommended by the CDC for our analyses. Additional project details have been described elsewhere (310).

\section{Study population}

Our analysis excluded participants who were $<18$ years of age, pregnant, senile, had a stroke and used a proxy to complete the interview, or had current cancer; those missing data on key covariates were also excluded, as were participants with extreme values for exercise $(>6,000$ minutes per week) in order to eliminate potential information bias; these were the only extreme values considered for exclusion. Further exclusion of persons with missing data on FMS and herbal use (at 30 days, 12 months, or ever using herbs) yielded final study samples of 20,127 and 30,672 adults for 2007 and 2012, respectively (Figure $1)$.

\section{Variables}

Outcome Variables. The main outcome variables for 6 separately run analyses were (in three models for each of the two survey years) the use of herbal supplements within the previous 30 days, 12 months, and having ever used herbal supplements (all dichotomous variables); these included herbs in the form of pills, capsules, tablets (2007) or liquids (2012), and excluded herbal or green teas.

Exposure Variable. Our main exposure was Fibromyalgia Syndrome (FMS) diagnosis, a dichotomous variable, which was self-reported as a previously diagnosed condition by a health professional.

Covariates. Demographics lifestyle factors, health conditions, and related factors known or suspected to be associated with CHAs, herbal use, and/or FMS were considered a priori as potential covariates in multivariable models. Demographic factors assessed included age, gender, race/ethnicity, education, employment, income, marital status, geographic region, and place of birth. Additional related factors included insurance status, family out-of-pocket medical costs in the past year, and delay of care due to 
concerns over cost. Lifestyle factors included smoking status, alcohol use, exercise, BMI (21), health status, and substance abuse. Health conditions included self-reported history of physician diagnosed diabetes (27), kidney disease (24), gastrointestinal disorder $(24,26)$, respiratory conditions (22), dyslipidemia, liver condition, Rheumatoid arthritis, Cardiovascular disease (24), hypertension, nonspecific arthritis (23), gout, migraines (29), mental health condition, insomnia, and previous cancer diagnosis. These variables are described in greater detail below.

Potential Effect modifiers. Gender (Male/Female) and number of health conditions (0-1/2+) were assessed for their potential influence on the relationship between FMS and herbal use.

\section{Statistical Analysis}

We conducted complete-case analyses using SAS 9.4 (Cary, NC, USA). We merged NHIS Family, Person, Household, Sample Adult, and Adult Alternative health files for each year, and measured sample characteristics, including frequencies and percentages/prevalence rates of herbal use (ever, past 12 months, and past 30 days) for 2007 and 2012, respectively (using PROC SURVEYFREQ and PROC SURVEYMEANS); we extrapolated estimates to generate population frequencies using NHIS sampling weights. Weighted t-tests (PROC SURVEYREG) and Rao-Scott Chi-square tests were used to determine significant differences by herbal use status for three herbal use time points in both survey years; significant factors were included in final models, for which we assessed the influence of potential confounding factors. Weighted logistic regressions (PROC SURVEYLOGISTIC) were used to evaluate the independent associations of FMS diagnosis to herbal use in the last 30 days, the last 12 months, or ever. We also used a DOMAIN statement to keep our exclusions separate while maintaining the integrity of sampling weights. We used Chi-square and t-tests to determine significant changes between 2007 and 2012 on weighted frequencies and means of all items, in addition to specific herbs used, herbal practitioner visits, physician disclosure of herbal use, and use of other CHAs; we considered trends between time points significant if there was no overlap in weighted percentage confidence intervals. Differences between participants missing any data were assessed using logistic regression. All p-values shown are two-sided at $\mathrm{p} \leq 0.05$.

For 2007 and 2012, we examined crude associations between FMS and herbal use at 30 days, 12 months, and any previous use of herbs. Three additional models for each year adjusted for demographic and related covariates, followed by six total models additionally adjusting for lifestyle factors. Last, we additionally controlled for health conditions as well, in six (full) models.

Our models included demographic characteristics of age (evaluated as both continuous and in groups 18-24, 25-44, 45-64, 65-74, and 75+ years), sex (Male/Female), race (defined as 'Non-Hispanic White,' 
'Non-Hispanic Black,' 'Hispanic,' 'Asian,' and 'Other'), education (a categorical variable defined as '<Some HS,' 'HS/GED,' 'Some college/AA/tech,' and 'Bachelor's Degree+,'), employment ('Employed for pay,' 'Employed but not for pay,' and 'Unemployed,'), income (\$1 - \$24,999; \$25,000 \$44,999; \$45,000 - \$74,999; \$75,000+; 'Don't know'; and 'Missing'), and geographic region ('Northeast,' 'Midwest,' 'South,' and 'West'), and place of birth (U.S.-born/other), which were controlled for in a multivariate model first, as were related factors insurance status (separate categories 'Uninsured,' 'Medicaid,' 'Medicare,' 'disability,' and 'private'), family out-of-pocket medical costs in the past year (aside from insurance premiums, deductibles, and/or co-pays) ('None;' $<\$ 500 ; \$ 500-\$ 1999 ; \$ 2000$ \$2999; \$3000-\$4999; \$5000 and over; and, 'Don't know'), and delayed access to care because "could not afford” or "worried about cost" (yes/no). Marital status (evaluated as 'married or co-habitating,' 'divorced/separated/ widowed,' and 'single') was additionally controlled for in 2012 models. Models also controlled for lifestyle factors BMI (evaluated as continuous and categorical variables using the National Institutes of Health clinical classifications scores of <18.5 - 25='Underweight or Normal weight;' 25 29.9='Overweight;' 30 - 34.9='Obese Class 1;' and, 35+='Obese Classes 2/3') (295), tobacco use (defined as 'current smoker,' 'former smoker,' and 'never smoked'), alcohol use ('None,' 'Light,' and 'Moderate to heavy'), substance abuse (other than tobacco or alcohol) in past year (yes/no), physical activity of at least 10 minutes per week (continuous and dichotomous as 'Any'/'None'), and self-reported health status ('Excellent/very good/good,' 'Fair,' and 'Poor').

In our fully-adjusted models, we also evaluated the influence of both specific health conditions and total number of health conditions on the relation of FMS to herbal use. A comorbidity index was created from 0-13 participant-reported conditions; these included history of 1) diabetes (27);2) kidney disease (24); 3) gastrointestinal disorder (including history of ulcers, inflammatory/irritable bowel disease or constipation severe enough to require medication in the past year and/or (2012) abdominal pain, digestive allergy, and/or heartburn in the past year) $(24,26)$; 4) respiratory conditions (history of asthma or emphysema, and/or chronic bronchitis) (22); 5) dyslipidemia; 6) liver condition; 7) rheumatoid arthritis; 8) cardiovascular disease (coronary heart disease, angina, and/or heart attack) (24); 9) hypertension; 10) non-specific arthritis (23); 11) gout; 12) migraines (29); and, 13) mental health condition (depression, phobias, and/or often anxious in past year, and/or ever having bipolar disorder). We categorized this index as 'No health conditions,' '1 condition,' 2 conditions,' and '3 or more conditions'; Health conditions were evaluated both collectively (as continuous and categorical variables) and individually; insomnia in the past year or previous cancer diagnosis were also evaluated individually.

We assessed the potential modifying influence of gender and number of health conditions $(0-1 / 2+)$ on the association between FMS and herbal use at each outcome time point and for each survey year; 
interactions were assessed by including the corresponding multiplicative-interaction term in crude statistical models.

\section{Results}

Relative to participants with complete data in both survey years, those employed and with higher education were less likely to have missing data on key covariates ( $p$ 's $\leq .0001)$. Those more likely to have missing data were underweight, older, and with poor reported health status ( $p$ 's $\leq .0001)$; in 2012 , insured women were also more likely to have missing data $(p \leq .0001)$. Participant age ranged from 18 to 85 years, averaging $47.5(\mathrm{SE}=0.25)$ and $48.5(\mathrm{SE}=0.18)$ years for 2007 and 2012 , respectively. In both survey years, study participants were predominantly white (72.2\% and 69.5\% for 2007 and 2012, respectively), female (53.1\% and 53.5\%), and insured (84.3\% and $84.2 \%)$; most (56.4\% and 57.9\%) reported never smoking. Nearly one-third of participants were overweight (mean BMI for 2007 BMI = $27.3 \pm 0.05 ; 2012=27.7 \pm 0.04)$, and most $(64 \%$ and $72.3 \%)$ reported at least one chronic health condition, with the number of chronic health conditions increasing from a mean of $1.5 \pm 0.02$ in 2007 to $1.9 \pm 0.01$ in 2012 .

Demographic and lifestyle characteristics are displayed in Tables 1a (any previous use of herbs),

2a (herbal use in past 12 months), and 3a (herbal use in past 30 days), while Tables $1 \mathrm{~b}, 2 \mathrm{~b}$, and $3 \mathrm{~b}$ display health characteristics, respectively. Those reporting herbal use (ever, and past 12 months) significantly decreased between 2007 and 2012 (p's <.0001). Those with any herbal use (ever) comprised 27.1\% $(2007 \mathrm{n}=5057)$ and $24.2 \%(2012 \mathrm{n}=7328)$ of population samples (Table 1a), with decreases in these prevalence rates for recent use of herbs (12 months: $2007 \mathrm{PR}=19.1 \%$; $2012 \mathrm{PR}=$ $16.4 \%$ ) (Table 2a); nearly 14\% used herbs within the past 30 days $(2007 \mathrm{PR}=13.9 \%$; $2012 \mathrm{PR}=$ $13.9 \%$ ) in both survey years (Table $3 a)$.

In 2012, fewer whites consumed herbs (ever, past 12 months) for health benefit compared to 2007, while the Hispanic population increased their use (p's $=<.0001)$. Herbal users exercised more and smoked less in 2012 than in 2007 (p's $=<.0001$ ). Additionally, herbal use was associated with a significant increase in out-of-pocket costs in both survey years (p's for trend $=.0001$; not shown); specifically, there was a significant increase in out-of-pocket costs over $\$ 3,000$ among herbal users (ever, past 12 months) between time points. There was a significant decrease in privately-insured herbal users (ever), and an increase in herbal users (past 12 months) with Medicaid (p's < .0001). All herbal users had significantly higher numbers of health conditions compared to non-herbal users in both 2007 and 2012 (p's for trend $=<.0001$; not shown). Rates of chronic pain conditions (including migraines, non-specific arthritis, gout, non-specific joint pain last greater than 3 months, and/or Rheumatoid 
arthritis) and mental health conditions among herbal users (past 12 months, past 30 days) was also significantly higher in 2012 than in 2007 (p's $=<.0001$ ) between time periods. Lastly, consumption of natural products increased among all herbal users between time points, while significant decreases occurred in those using herbs and reported ever using manipulative therapies/bodywork, as well as alternative medical systems (Acupuncture, Ayurveda, homeopathy, naturopathy) (p's < .0001).

Prevalence of diagnosed FMS also increased significantly from $1.3 \%$ of adult participants in 2007 to 1.7\% in 2012 ( $\mathrm{p}<.0001$ ) (Table 4). However, consistent with overall trends in herbal use (Table 1a-3a), herbal use (ever) among adults diagnosed with FMS declined significantly from 2007 to 2012, decreasing from 57 to $41 \%$ ( $\mathrm{p}<0.0001$ ); however, there were no significant differences in the proportions of those with FMS using herbs in the past 12 months (42\% to $31 \%$ ) or 30 days (33\% to $25 \%$ ) (Table 4 ). There were no changes between time points in herbal use disclosure rates to physicians $(64.6 \%$ within the past 30 days in 2007 to $71.4 \%$ within past 12 months for "top herbal supplements used") and number of different of herbal supplements taken in the past 30 days $(2007$ Mean $=0.99, \mathrm{SE}=0.20$ vs. 2012 Mean $=$ $1.1, \mathrm{SE}=0.38 ; \mathrm{p}=.89$ ) among those with FMS.

Relation of FMS to Herbal use. Nearly $60 \%$ of those with FMS had previous/current use of herbs (ever), while $42 \%$ used herbs in the past 12 months, and 33\% used herbs in the past 30 days.

Approximately 3\% of herbal users (ever, past 12 months, past 30 days) had FMS in 2007 and 2012; this rate did not differ between time points (Table 5). Relative to those without FMS, participants diagnosed with FMS were over three times as likely to use herbs for any length of time in 2007. After adjustment for demographic, lifestyle, and health factors, those with FMS were 2.7 times more likely to ever use herbs than those without FMS (Adjusted odds ratio $(\mathrm{AOR})=2.66,95 \%$ confidence interval $(\mathrm{CI}): 1.85$, 3.82); this positive association remained when examining the association between FMS and recent herbal use as well (12 month and 30 day AOR's = 2.25 (CI: 1.51, 3.35) and 2.34 (CI: 1.55, 3.52)). Likewise, FMS was significantly associated with all herbal use outcomes in 2012, although the magnitude of the associations were comparatively attenuated (2012 AOR's: herbal use (all) = 1.59 (CI: 1.25, 2.03); 12 month $=1.57(\mathrm{CI}: 1.21,2.04) ; 30$ days $=1.51$ (CI: 1.15, 2.00)).

As illustrated in Table 6, we found evidence of a modifying effect of comorbidity only for the relationship between ever using herbs and FMS in 2007; the magnitude of the association of herbal use to FMS was significantly greater in those with $0-1$ comorbid health condition than in those with 2 or more health conditions (OR's, respectively $=4.67$ (CI: 2.22, 9.81) vs. 2.80 (CI: 1.99, 3.92; p for interaction $=.008$ ); we found no evidence of a modifying effect of multiple comorbidities for either year, even after controlling for age. Additionally, we found no evidence for a modifying effect of gender on the relation between FMS and herbal use in either year. 


\section{Discussion}

Our study was the first large, population-based study to examine the relation of herbal use to FMS in any population, while controlling for a wide array of potential confounders and examining the modifying influence of comorbid conditions; it is also among the first rigorous studies to investigate the relation of herbal use to any chronic pain condition in a large sample. Relative to adults without FMS, those with FMS were significantly more likely to use herbs at 30 days, 12 months, or ever, in both 2007 and 2012 survey years; these positive associations remained highly significant even after controlling for a broad array of demographic, lifestyle, and health-related factors.

In our study, $60 \%$ of those with FMS had ever used herbs, while $42 \%$ used herbs in the past 12 months, and 33\% used herbs in the past 30 days. Studies assessing herbal use in FMS have not examined relationships as we did, but only reported frequencies of use; these ranged from $6.8 \%$ (in a large sample of Wisconsin primary care patients receiving opioids) to 78\% (among 90 FMS patients in the UK) $(93,196,197)$. However, these studies were limited by combining herbs with other natural products $(14,93,196,197,311)$ in proportion estimates, and the wide variation in proportions likely arise from using different definitions for herbs and supplements. When examining herbal use in rheumatoid arthritis populations, $20 \%$ - 35\% used herbal medicine; however, these small ( $\mathrm{N}=78$ and $\mathrm{N}$ =94) studies were conducted over three decades ago (193-195).

Prevalence of diagnosed FMS was significantly higher in 2012 compared to 2007 (1.7\% vs. $1.3 \%$ ), which is slightly less than national estimates (1.75\% of those in the U.S. (8)) but fits within prevalence ranges $(0.5-5 \%)$ of developed countries (7); this apparent rise is likely due in part to increased recognition of FMS by physicians after updated 2010 ACR criteria, potentially enabling shorter patient waiting times to diagnoses than before.

In contrast, we found reported herbal use among adults with FMS to decline significantly from 2007 to 2012; this decrease may in part reflect the modest but significant reduction in overall herbal use observed between survey time periods. Additionally, the decrease in odds of herbal use in FMS may also reflect the availability of FDA-approved FMS treatments that became available in 2007, 2008, 2009 shortly following implementation of the 2007 NHIS survey.

It remains possible that both the increase in FMS diagnoses and the decrease in odds of herbal use for FMS over time are also in part perhaps the result of corporate promotion for FMS drugs, increased off-label use of other drugs for FMS, and potentially, increased opioid use for FMS. Between 2007 and 2012, the mean number of different herbal supplements used by those with FMS did not differ, and both means $(2007$ mean $=0.99 ; 2012$ mean 1.07) were lower than that of a 2009 North- 
American case-control study $($ FMS $=434$, Control $=198)$ which showed those with FMS took $~ 2.8$ per person; however, this average was also combined with the use of all other natural products (197). Ours is the first study to document the rates of FMS in herbal users, which remained consistent at $~ 3 \%$ in both 2007 and 2012.

The magnitude of the association between herbal use (ever) and FMS was significantly greater in those with 0-1 comorbid conditions in 2007 but not 2012. Although the possibility remains that this finding may indicate those diagnosed with FMS before 2007 were more likely to have used herbs in an attempt to exercise control over their health, since no FDA-approved treatments existed before 2007 data collection (312). While it remains possible those with FMS managed their illness more successfully with herbs, resulting in fewer FMS comorbidities before FDA-approved treatments were made available, these findings should be interpreted with caution.

Our ability to examine associations between herbal use and FMS in nationally representative samples that spanned two time periods comprehensive data on herbal use, as well as on many potential confounders, including demographic, lifestyle, health-related, and other factors, are major strengths of our study.

However, there were some limitations, including inadequate capture of certain herbal products. No data were available on consumption of herbal teas used for health purposes or on other herbal products not labelled as a "dietary supplement", including traditionally prepared herbal creams, tinctures/extracts, and powders, or products (with different labelling than that specified by the NHIS) that are sometimes recommended by herbalists over widely-available supplements due to quality and processing concerns. Additionally, many of the CHAs were only assessed in NHIS if they were administered by a practitioner (e.g., massage therapy, deep breathing only as part of other guided meditation practice, etc.). Our estimates for herbal use and other $\mathrm{CHAs}$ are likely underestimated due to misclassification of herbal use by the additional inclusion of non-herbal products including coenzyme Q-10, fiber, SAM-e, fish oil, and prebiotics/probiotics in the NHIS.

Additionally, the 2012 questionnaire differed substantially from 2007. Based on cognitive testing and input from expert panels, the definitions of certain modalities were modified between years to reduce false-positive responses (313), which may have affected findings. However, these changes were relatively minor and are unlikely to impact our estimates.

Given the high prevalence rates of those with FMS using herbs (as indicated through positive associations with all herbal use outcomes), additional observational studies are needed to explore potential impacts of herbal use on a variety of chronic pain conditions, in order provide rationale for clinical trials on potential herbal therapies for other poorly-managed chronic diseases. Future observational research should continue to examine patterns of herbal use, and clinical research should also examine the safety and efficacy of herbs. 


\section{Conclusion}

In this large cross-sectional study of two nationally representative samples of US adults, reported diagnosis of FMS was strongly and positively associated with any duration of herbal use in both 2007 and 2012 after adjustment for demographic, lifestyle, and health factors; As a result of this study, we have an increased understanding of the patterns of herbal use prior to and following the implementation of an update to FMS diagnostic criteria and increased availability of treatments for a disease characterized by chronic pain. Rigorous prospective studies are needed to confirm these findings, to further explore the patterns and determinants of herbal use in other chronic disease populations. 


\section{CHAPTER 4: Correlates of Herbal Use and other Complementary Health Approaches among Appalachian Pain Patients}

\section{Introduction}

Over $20 \%$ of adults eventually experience chronic pain $(2,3)$, and as many as 100 million individuals are affected in the United States alone (4). The International Association for the Study of Pain (IASP) defines pain as chronic if it persists beyond normal tissue healing time of 3 months (314). Unrelieved pain results in decreased function, longer hospital stays, and increased rates of rehospitalization and outpatient visits, leading to lost employment, income, and insurance coverage (5). Chronic pain costs the U.S. more than $\$ 635$ billion annually in medical costs and lost productivity, which equates to approximately $\$ 2,000$ per person (3).

The Institute of Medicine stresses the importance of increased research regarding the translation of effective treatments into practice and regarding the prevalence and costs associated with pain (6). While many pain measurement tools exist (315-324), some instruments incorporate frequency and intensity criteria, while others do not; this adds to variation in prevalence estimates $(1-3,325)$. Recently, pain physicians and researchers gathered to create a more standardized measurement tool for pain assessment, the Global Pain Scale (GPS) which incorporates elements of existing pain measurement tools and measures various clinical and quality of life factors (315).

Poor pain management frustrates patients and their caregivers; poor symptom management often prolongs pain treatment, and many of these treatments carry significant side effects (326). The identification of superior management options for chronic pain is difficult, likely due to the multiple complex mechanisms and signaling pathways through which chronic pain is generated $(30,31)$; many chronic pain patients thus experience poor treatment outcomes (2). Chronic pain is challenging to manage, partially due to limited efficacy and negative side effects from conventional medical therapies, but also because some physicians are reluctant to prescribe opioids (6), while others overprescribe them (85).

Over the past decade, the worrisome trend of increased prescription and utilization of opioids for the treatment of chronic nonmalignant pain has emerged (83), even though opioids cause physical and psychological dependence in users. Additionally, there is no concrete evidence opioids are effective in treating some chronic pain conditions for which they are prescribed $(18,75)$. Opioid pain relievers-also called prescription painkillers - such as Oxycodone, Hydrocodone, Fentanyl, and Hydromorphone, were responsible for three-fourths of all prescription drug overdose deaths in the United States in 2010 (84). Nationally, deaths involving opioids (using age-adjusted data) have more than quadrupled since 1999 
(84). However, non-opioid medications such as Nonsteroidal Anti-Inflammatory Drugs (NSAIDs) carry significant side effects for as many as $25 \%$ of long-term users (62).

Opioid treatment for chronic pain management presents a great challenge in the unique demographic of Appalachia. In particular, West Virginia, the only state situated entirely within Appalachia, ranks among the highest in the nation for several health disparities, including opioid pain medication misuse and abuse. West Virginia's overdose death rate has been climbing (28.9 per 100,000 population in 2010, compared to 35.5 per 100,000 in 2014), and was more than double the national rate, which has also been rising over time (12.4 per 100,000 population, compared to 16.1 per 100,000, respectively) $(86,327)$.

Understanding the patterns of usage and efficacy of other, safer forms of chronic pain treatment are necessary, and may include Complementary Health Approaches (CHAs) such as herbal medicine. Some patients choose CHAs because of concerns over the safety of conventional medicine (100); Painrelated disorders are the most commonly reported conditions for which patients use CHAs $(86,88$, 93, 105, 106); CHAs encompass therapeutic approaches not customarily prescribed by medical doctors to treat chronic pain, such as natural products (e.g., herbs, probiotics, fish oil) and Mind-body practices (e.g., yoga, meditation, etc.), as well as Ayurvedic medicine, Traditional Chinese Medicine, Naturopathy, homeopathy, and other practices. Most people use CHAs with conventional treatments $(90,95,96)$, but patients often do not disclose the use of CHAs to their physicians $(97,98)$. CHA use is popular in the United States, with 30-60\% of the general population estimated to use CHAs (88). In 2007, about $\$ 14.8$ billion was spent on the purchase of non-vitamin, non-mineral, natural CHA products in the United States (e.g., herbs, fish oil, etc.) (109). The most comprehensive systematic review to date (28 trials meeting quality criteria and presenting an economic evaluation) concluded that substituting a complementary and integrative medicine (a.k.a. self- or practitioner-administered CHA) care component in place of usual care had better health outcomes and lower costs than usual care alone for a variety of chronic health conditions (328). However, comparative research is largely lacking, as is research regarding the overlap of CHA use and/or conventional treatments among those with pain conditions (329).

Herbs (plant-derived products also known as 'botanicals', 'biologically based therapies', and 'herbal medicine') are sold as food and dietary supplements in the United States. In 2012, 17.7\% of U.S. adults had used a dietary supplement other than vitamins and minerals in the past year, making these products the most popular CHA (104).

Herbal medicine is also part of many 'folk medicine' traditions. Anecdotal accounts suggest that many herbs have been recommended by herbalists for the treatment of pain, rheumatism, and related symptoms (121, 179, 180, 211, 216-219). The history of Willow (Salix spp.) and other salicin-rich plants, which are pre-cursors to the NSAID Aspirin, is an example of the role herbs have played in pain 
management (123). A meta-analysis of Capsicum (a compound extracted from the fruit of Chili peppers C. frutecens and others) for the treatment of pain, reported that topical Capsaicin was superior to placebo for the treatment of chronic pain from neuropathic and musculoskeletal disorders (with trials of moderate to high quality); the FDA approves Capsaicin as an OTC medicine as topical cream (199). There is also extensive anecdotal evidence indicating widespread use of botanicals for the relief of pain. Based on current anecdotal evidence, plants with analgesic, anti-inflammatory and/or anti-spasmodic activities continue to be used as well.

However, there are relatively few population-based studies assessing the prevalence and/or correlates of herbal use in pain populations. A large cross-sectional study among Wisconsin primary care patients receiving opioids as a primary treatment method for chronic pain showed that $6.8 \%$ used herbs and supplements (93). However, a cross-sectional study of primary care patients with chronic pain conducted in 12 academic medical centers demonstrated a higher prevalence rate (15\%) for herbal use (95). Additionally, two studies indicated herbal use prevalence among U.K. Rheumatoid Arthritis patients varied from $20 \%(\mathrm{~N}=78)(193,194)$ to $35 \%(\mathrm{~N}=94)(194,195)$ of used herbal medicines, while $78 \%$ $(\mathrm{N}=90)(194,196)$ of U.K. FMS patients used dietary supplements (including herbal supplements).

The history of folk medicine in Southern Appalachia is much better known in some areas than others; more research has been conducted in western NC, eastern TN, and eastern KY. Comparable research has been conducted more recently in other areas, particularly northern Georgia, southwestern VA, mid-TN, western KY, and northern Alabama (124). West Virginia has a known anecdotal history of herbal medicine use, and some of it is specific to folk illnesses as part of Appalachian folk medicine (169, 217); however, no epidemiological studies on herbal use have been conducted in WV pain populations. There are many methodological problems attending the gathering of information on the use of folk medicine, including small sample sizes, misclassification bias (investigators have failed to distinguish between past and present herbal medicine usage), and failure to account for contextual factors influencing use, including concurrent use of conventional medicines (124).

In a comprehensive literature review of herbal texts and qualitative studies of herbal use in Appalachia (Appendix B), we found anecdotal evidence for the historical use of 101 herbs for pain relief $(121,179,180,216-219)$, rheumatism $(121,169,178-180,182,191,216-218)$, and arthritis $(121,169$, $178,179,191,212,217,218) ; 12$ herbs for non-specific inflammation $(121,179,180,217,218)$; 3 for alleviating sleep troubles $(121,169,179)$; and 4 for headache $(179,180,217)$. It is not clear if these herbs continue to be used for these conditions in Appalachia. Unfortunately, rigorous observational studies and high-quality trials regarding the use and efficacy of herbs for chronic pain syndromes remain sparse and little is known regarding the correlates and motivators of herbal use, including pain level and prescription drug use. Clearly additional research is needed, particularly in Appalachia, where prevalence of chronic pain disorders (such as arthritis (330)) is high and opioid misuse is epidemic. 
In this study, we examine potential motivators of herbal and CHA use by using a Transtheoretical Model-guided tool to better understand the underpinnings of short-term and long-term use. The Transtheoretical Model (TTM) is a behavioral theory which proposes that changes in a health behavior consist of movements between sequences of discrete, qualitatively distinct stages, characterized by distinct mindsets. As individuals move between these stages, specific social-cognitive factors influence stage progression (331); stage progression occurs through 6 separate 'Stages of Change' (Figure 1): Precontemplation (a person has no intention to change a health behavior), Contemplation (they begin to consider changing a health behavior), Preparation (they intend to change this behavior), Action (initiation of the new health behavior), Maintenance (execution of the health behavior for more than 6 months) (332), and the newly added Termination (desired health behavior complete) (333). The 'Stage of Change' construct is the central organizing dimension of the TTM (334); this construct has demonstrated success in measuring various stages of health behavior change in cross-sectional research on the topics of diet $(335,336)$, the use of health care proxies (337), exercise in pain patients (338), physical activity and body satisfaction (339), and measuring health-promoting behaviors for cancer prevention in a healthy population (340). However, the TTM Stages of Change construct has never been applied to measure the health behavior of herbal use, nor other CHAs.

Current and future public health research calls for the use of theoretical models to better understand motivators of health behaviors (341) such as herbal medicine use; social/biobehavioral underpinnings in research are critical to understanding the processes and motivators of health behavior change. The use of a theoretical underpinning/conceptual framework, such as the TTM Stages of Change construct, in assessing the correlates of herbal medicine use (and the use of other CHAs) can help elucidate the reasoning and potential motivators behind an individual's decision-making process regarding the use of these approaches.

The use of herbs and other CHAs may signal a patient is actively engaged in a potentially positive health behavior, but may carry a risk of potentially negative interactions with other treatments. Gaps in existing research highlight a need to examine correlates of herbal use, as well as use of a variety of CHAs, to better understand if pain and other factors may influence individuals to engage in these health behaviors. Additionally, gaps exist with regard to identification of herbs used in Appalachia for pain, for which knowledge of potentially superior pain treatments may be lost; further investigation is also needed regarding the relation of herbal and other CHA use to demographics, lifestyle characteristics, pain and other health conditions, and other health-related factors, including prescription drug use. By employing a new, comprehensive instrument for measuring pain and CHA use and incorporating questions regarding a broad range of potential correlates, this cross-sectional study was designed to help address these gaps. 


\section{Methods}

\section{Scope of Project}

This cross-sectional survey study was conducted from June 2014 - March 2016 in a sample of 301 English-speaking adult ( $\geq 18$ years) patients in four Northern West Virginia pain and rheumatology clinics. We conducted primary data collection using a new measurement tool, the Complementary Approaches for Pain Survey (CHAPS) (Appendix D), using consecutive sampling (Response rate across clinics $=88.0 \%$ ), in order to investigate the associations between a variety of demographic, lifestyle, health, and pain correlates with herbal use and other CHAs for pain in an Appalachian pain patient population. Details regarding survey development and content, study population, and survey administration are given below.

\section{Survey Development}

Development. The CHAPS included 2 closed-ended items regarding past and present chronic pain; demographics and lifestyle (10 and 6 items, respectively); 24 health conditions (including nine known to cause pain) and other health factors (3 items); the Shortened-Form Global Pain Scale (SF-GPS) pain assessment (20 items), and, open-ended questions regarding specific herbs and other CHAs (2 items). Additionally, we assessed the usage of 12 separate CHAs, including Herbs/Botanicals, following the definition of 'Herbs' from the National Institutes of Health's (NIH's) National Center for Complementary and Integrative Medicine (NCCIM), which consider herbal products a CHA falling into the subgroup of 'Natural products'. Natural products listed on our survey instrument included 'Herbs/botanicals,' 'Vitamins and minerals,' 'Probiotics,' and 'Other Natural products;' accompanying examples (e.g., "Herbs/Botanicals (such as Echinacea, Black Cohosh, etc.)"), were also included to minimize misclassification bias among users who may have been confused. These questions were asked how long they had used each CHA; length of time corresponded to Stage of Change $(334,341)$; for each CHA, this included: "I do not know what this is;" "No, and I do not intend to within the next 6 months (Precontemplation stage);" "No, but I intend to within the next 6 months (Contemplation stage);" "No, but I intend to within the next 30 days (Preparation stage);" "Yes, and I have for less than 6 months (Action stage);" "Yes, and I have for more than 6 months (Maintenance stage);" and, "If Yes, how effective is it in managing your pain?". Twelve optional, nested questions regarding the efficacy of CHAs (if used) were also included.

Stage of change questions were constructed based on previous surveys measuring health behaviors by the TTM Stages of Change framework $(334,341)$. Researchers have indicated that projects 
applying TTM should include investigation of problem severity (341). Our approach to measuring problem severity among this WV pain populations was through the use of the SF-GPS (315).

The SF-GPS (315) was adapted from the pre-validated Global Pain Scale (GPS) by its original authors, and was used in this research; The GPS was created to meet the need for a simplified instrument for use by both clinicians and researchers, incorporating key elements of many existing pain assessments. The GPS uses a variation of the Visual Analog Scale (VAS); numbers are spaced equidistant from one another on a horizontal line, both ends are defined as the extreme limits of the parameter measured (pain severity, etc.), and orientation is from the left (best) to the right (worst), indicating the impact each measure has had in a participant's life. The SF-GPS contains 5 items each on pain, feelings, clinical outcomes, and activities; it addresses the ceiling, floor, and average pain over the past week, as well as current pain state; screens for depression, anxiety, fear, hopelessness, and energy level, while exploring the effect of pain on respondents' quality of sleep, comfort, medication consumption, mood, independence, energy, work interference, perceived control over pain, health care utilization, and satisfaction with health care received. Finally, it also assesses how pain affects respondents' ability to complete a variety of activities.

A cover page was attached to every survey, indicating that the study was open only to patients over the age of 18 years, ensuring anonymity, and indicating the intent of the research study (Appendix D); returning of the survey served as implied informed consent. The CHAPS was designed to require no more than 15 minutes to complete, and the average time for survey completion among clinic patients was 12 minutes.

Pre-testing/Pilot period. Following initial development of CHAPS and after obtaining provider permissions and IRB approval, we piloted the survey in a convenience sample of pain patents attending the WVU Medicine Pain clinic in Morgantown, WV (June 2014 - March 2015). We visited the clinic waiting room in person to solicit patient participation in the survey. In addition, we also placed a stack of surveys, pens, and a survey drop box in the waiting room; during the following 9 months, we visited every 2-3 weeks to collect surveys, and ensure surveys and pens were replenished. A total of 66 patients completed surveys during this period, including 28 during our in-person visit.

A preliminary version of the CHAPS was also pre-tested in 10 patients by two independent investigators in order to assess comprehension, recall, identification of strong and weak points, and through gauging sensitive/controversial elements (342); our investigators gauged this through the assessment of 13 pre-determined questions. Wording of the survey was designed to be at a ninth grade reading level or below.

Based on responses to and feedback on this preliminary survey, and after learning more about the patient experience through a 20-hour clinical immersion (accompanying a lead physician in patient rooms 
at the WVU Medicine Pain clinic), we made minor formatting changes to the CHAPS to improve clarity, and included an additional question about (each) prescription use, CHA efficacy (if respondent used a CHA), and military status. We then pre-tested the final survey version in 11 additional patients at the same clinic location.

Final Version. Minor alterations were made to the final version of the CHAPS by September 2015. This 89-item self-administered survey required 10-12 minutes to complete. The survey included questions regarding use of a variety of CHAs, including herbs, in a manner consistent with surveys utilizing a Stages of Change approach (335, 337-339, 343). The CHAPS also included the 20-item SF-GPS(315); questions regarding a variety of demographic, lifestyle, and health factors (10, 6, and 25-items, respectively); and, 4 open-ended questions regarding additional $\mathrm{CHA}$ use, diagnoses, and prescriptions used for pain. There were no differences in CHA/herbal use outcomes by survey version (Fisher's $\mathrm{p}=$ .102 and .690 , respectively) or (pilot) test period (Fisher's $\mathrm{p}=.879$ and .678 ). Based upon the similarity of the preliminary and final CHAPS surveys, data from these surveys were pooled in our primary analyses.

\section{Data Collection}

Target study population and Identification of Sampling Locations. Our target study population comprised adult chronic pain patients attending a WV pain management or rheumatology clinic within a 100 mile radius of Morgantown, WV. Pain and rheumatology clinics were identified by conducting an internet search utilizing the Bing search engine; an online White Pages directory (www.whitepages.com); and, online healthcare specialist directories (www.healthgrades.com; www.RA.com; and, www.vitals.com) with search terms "Rheumatology," "Rheumatology clinic," or "Pain clinic," and "WV," or "West Virginia" and (for internet specialist directories) using the 'Physician locator' function to search for rheumatologists within 100 miles of the CHAPS office (May - July 2015).

Of the 15 and 91 rheumatology and pain management practices identified, respectively, non-pain specializing physicians were excluded, as were multidisciplinary practices that included specialties other than pain or rheumatology (Figure 2); also excluded were eye doctors, podiatrists, neurosurgeons, chiropractors, acupuncturists, and weight centers, leaving 9 and 30 eligible rheumatology and pain clinics, respectively. After exclusion of duplicates and those not meeting location criteria, we made phone calls to all remaining clinics ( 6 and 9, respectively) to verify address and purpose of the business, asking "Is this a rheumatology/pain management clinic, and do a majority of patients seek care in your facility for pain?". Following a positive response from rheumatology and pain clinic personnel, we 
engaged clinic managers or lead physicians from each facility in an effort to describe the study and secure permission to administer the survey to their patients. When clinic managers were not available to speak by phone or email, we attempted to follow up 3 times. A total of 4 eligible clinics ( 1 rheumatology and 3 pain management) located in 4 different WV counties agreed to participate. Managers from each participating clinic signed a consent form which: briefly described the study purpose, stated that study investigators would neither disrupt clinic practice nor provide any medical advice; and, provided the IRB number and the primary contact information for the CHAPS study coordinator. Our resources allowed 24 total clinic visits throughout the study.

Clinic visits. Although our presence was not specifically advertised in clinics, we chose our seating in strategic areas of clinic waiting rooms (corners and/or areas located close to the entrance) so that we could utilize table space to hold our equipment (a number of clipboards, pens, and a stack of surveys). While we dressed nicely (i.e., no jeans or sneakers), we omitted the use of uniforms or formal business attire because we did not want to draw attention to our non-patient status. During clinic visits, a member of our team consecutively approached every patient (with the exception of one, who was on a stretcher with his face covered) by (in order) verifying patient status, giving a self-introduction and description of the study purpose, and asking for their participation as an anonymous respondent. When approaching someone in obvious pain, we added "I understand if you are in too much pain, or if you are not interested in filling out a survey." If they agreed to participate, we handed them a clipboard, survey, and pen; we thanked them regardless of decision. All surveys were self-administered with the exception of 14 (4.7\%), which were completed by a proxy; we also assisted with personal interview format requests $(\mathrm{n}=6,2.0 \%)$. Upon submission to a CHAPS team member, all surveys were marked with date and clinic location, folded to conceal any written information, and placed in a bag beneath our sitting area for delivery to the CHAPS office for analysis. All surveys were placed in a locked filing cabinet in our research coordinator's office when not in use by the CHAPS team. All data entry and compilation of a master codebook was completed by TF; data were checked periodically for data entry errors by visual examination, assessment of the distribution of each variable, and by examining the consistency of related variables.

During all phases of CHAPS research, we also engaged with clinic administrative staff, ensuring they were familiar with our study objectives; this enabled the return delivery of surveys taken back into the patient rooms. We used phones and other technology only minimally in waiting rooms, engaged in dialogue with patients when prompted, and kept a record of distance traveled and participant refusals. Public notification was not given for this study.

No participant incentives were used for this research study. Approval for the study was obtained from West Virginia University Institutional Review Board (IRB\# 1403248198). 


\section{Variables}

Outcome Variables. The main, dichotomous outcome variables for these analyses are 1) Current herbal use for pain (defined as 'Action [ $<6$ months] or Maintenance [ $\geq 6$ months] stage of herbal use' or 'Does not use herbs'); and, 2) Current use of other CHAs for pain (defined as the 'Action or Maintenance stage of CHA use' or 'Does not use CHAs'), which included the use of vitamins and/or minerals, probiotics, other natural products (excluding herbs), acupuncture, massage therapy, spinal manipulation/Chiropractic, Tai Chi, yoga, meditation, other relaxation practices, and/or movement therapies.

Independent Variables. We examined relationships between the outcomes with a variety of demographic, lifestyle, and health exposure variables in separately run models for each exposure. Demographics: variables included age, gender, race/ethnicity, education, marital status, household Income, body mass index, and employment status.

Lifestyle factors: Smoking status, alcohol use in past year, and exercise were included as lifestyle factors. Health factors: The SF-GPS score was our overall indicator variable for pain. Additionally, twenty-three comorbidities were assessed, as was past/current prescription medication use for pain management.

Covariates. We adjusted for education, age, exercise, SF-GPS, and health conditions in models, when sample sizes allowed.

\section{Statistical Analysis}

We conducted analyses using SAS 9.4 (Cary, NC), and measured means/frequencies of sample characteristics, including outcomes $\mathrm{CHA} /$ herbal use by each length of time used (Stage of Change). We manually coded the answers to open-ended questions describing specific herbs used for pain and generated frequencies for each. Next, we examined relationships between CHA/herbal use and a variety of demographic, lifestyle, and health factors among pain patients; we also assessed the potential influence of education and other significantly associated covariates from crude, individual models on CHA/herbal use for pain.

Demographic variables included age (continuous and 18-49/50+ years), gender (Male/Female), race/ethnicity (Non-Hispanic White/Other Race), education ('<HS/GED;' 'Some College/Associate/ Technical training;' ' $\geq$ Bachelor's degree'), marital status ('Single;' 'Divorced/Separated/Widowed;' 
'Married/Cohabitating'), and household Income (ordinal and in categories ' $\leq \$ 50,000$ ' $\$ \$ 50,001+$ '). Additionally, Body Mass Index (BMI, calculated as weight (pounds) x 703/height in inches ${ }^{2}$ ) was evaluated as continuous and categorical, using the National Institutes of Health clinical classifications (BMI scores of $<25$ = "Underweight or Normal weight;" $25-29.9=$ "Overweight;" $30-34.9$ = "Obese Class 1;" and, 35+ = "Obese Classes 2/3") (295), as was employment status (a categorical variable defined as 'employed,' 'not employed and not disabled,' 'disabled,' and 'retired'). Lifestyle factors included smoking status (defined as Current/former/Never), alcohol use in past year (None/any), and exercise (categorical as None/Any, and continuous, in 30-minutes per week increments).

Health factors included SF-GPS score, as well as comorbidities. The total SF-GPS score was our overall indicator variable for pain. As stated before, the SF-GPS contains 5 items each on pain, feelings, clinical outcomes, and activities; the total score is the sum of these subscales, and ranges from $0-100$, where $0=$ pain generally doesn’t affect life and $100=$ pain causes great distress in life) (315). Additionally, twenty-three comorbidities (a continuous index from 0-23, in addition to dichotomous variables: 'Pain syndromes,' which incorporated diagnoses of spine/back/neck pain, migraines, tension headaches, rheumatoid arthritis, osteoarthritis, temporomandibular jaw disorder, non-specific knee pain, fibromyalgia, and/or gout; 'Mental Health conditions,' including depression and/or anxiety; 'Injury,' including broken bones and/or musculoskeletal injury/tissue trauma; and, 'Other conditions,' for which hypertension, heart disease, irritable bowel disorder, renal disorder, asthma, chronic bronchitis, diabetes, cancer, stroke, and/or chronic fatigue syndrome were included). Past and current prescription medication use for pain management was manually recoded (None/any Rx) and examined as an exposure variable as well.

Additionally, we assessed the association of herbal use to concurrent use of other CHAs. Thus, we examined as independent variables CHA use (Current user of any CHA except herbs/None), Dietary supplement use (current use of Vitamins/Minerals, Probiotics, and/or other natural products excluding herbs/None), Mind and Body practices use (current use of Meditation, Relaxation, Yoga, and/or Tai Chi/Qi Gong/None), and, use of Acupuncture, Manipulative treatments, Massage, and/or Movement therapies (current user/None) in separate models. All CHA exposures were further examined in separate models by Action (short-term use, defined as $<6$ months) and Maintenance (long-term use, defined as $\geq 6$ months) Stages of Change for each respective CHA.

All multivariable models were adjusted for demographic and lifestyle factors that did not have a substantial proportion of missing values $(\geq 10 \%)$ and were associated $(\mathrm{p} \leq .10)$ with herbal/CHA use in the unadjusted analyses, such as education, chosen due to its established associations with herbal (144) and CHA use $(95,144)$. Additionally, we controlled for variables significantly associated with either 
respective outcome in crude models; these variables included age, exercise, SF-GPS, and health conditions, when adequate sample sizes for power allowed.

\section{Sensitivity Analysis of Missing Data}

As we had over $10 \%$ missing data on certain variables, including CHA use, herbal use, BMI, alcohol consumption, and income, we conducted additional sensitivity analyses using the fully conditional specification (FCS) method of multiple imputation (PROC MI/MIANALYZE); FCS was chosen because it allowed us to incorporate both dichotomous outcomes and all categorical/continuous predictors (with missing values ranging from 5.0\% - 53.5\%, for SF-GPS and those missing any of the 11 other CHA use questions, respectively) using a total of 250 imputed datasets. Prior to performing multiple imputation analyses, we first verified that data were missing at random by conducting regression tests on missing data for all variables of interest, followed by an assessment of missing data patterns to further verify data were missing at random (PROC MI). After imputation, we compared the unadjusted odds ratios between our imputed and original datasets; because no differences were detected, we have presented the results from our original dataset in the Results section.

\section{Results}

Response rates were high overall $(88 \% \pm 4.1 \%)$ ranging from 84 to $94 \%$. Common reasons for refusal to participate included hand pain, visual impairment (e.g., forgot glasses), or a reluctance to fill out paperwork. The average one-way travel time for patients across all locations was 74 minutes (ranging from 34 to and 129 minutes for pain clinics in Elkins and Morgantown, respectively). Results from our preliminary survey indicated very high internal consistency for the SF-GPS (Cronbach's alpha = .919); the internal reliability of each (5-question) subscale was also high (Cronbach's alpha for the Pain subscale $=.888$; Feelings subscale $=.874$; Clinical Outcomes subscale $=.804$; Activities subscale $=.904)$. Coefficients for the total sample were similar, likewise indicating high internal reliability of the SF-GPS (Cronbach's alpha overall $=.931$; Pain subscale $=.892$; Clinical outcomes subscale $=.830$; Activities subscale $=.908$ Feelings subscale $=.865)$.

Relative to participants with complete data, those missing data on any survey item were more likely to be older (Odds Ratio $(\mathrm{OR})$ for age $=1.04,95 \%$ CI 1.02, 1.07), prescribed opioids $(\mathrm{OR}=3.41, \mathrm{CI}$ $1.39,8.38$ ), and have a lower SF-GPS score (OR for lowest vs. highest SF-GPS quartile = 3.46, CI 1.62, 7.39). Those missing data on outcome variables $\mathrm{CHA} /$ herbal use were also older ( $\mathrm{OR}=1.04$, $\mathrm{CI} 1.02$, 1.06 and $\mathrm{OR}=1.04, \mathrm{CI} 1.01,1.07$, respectively) and more likely to use opioids (OR $=2.59, \mathrm{CI} 1.24,5.42$ and $\mathrm{OR}=5.42$, CI 1.92, 15.30) over no drugs. Although there was no difference in herbal use by clinic 
location (Fisher's $\mathrm{p}=.656$ ), there was a borderline significant difference in CHA use by location (Fisher's $\mathrm{p}=.05$ ), with the lowest use of CHAs (other than herbs) found among rheumatology clinic patients in our sample (4.0\%), compared to the highest proportion of other CHA use in the Morgantown pain clinic (78.5\%). There was no difference between those using a proxy/personal interview vs. selfadministered format (Fisher's $\mathrm{p}=.857$ ) by herbal use; however, there was a difference in survey format by CHA use (Fisher's $\mathrm{p}=.034$ ), with a lower CHA use proportion among those using a proxy or personal interview format (4.0\%) compared to those self-administering surveys (96.1\%).

Demographic and lifestyle characteristics are displayed in Table 1. Study participants were predominantly white $(92.0 \%)$, female (56.9\%), and married or cohabitating (57.5\%). Participant age ranged from 22 to 88 years, averaging $55.6(\mathrm{SD}=13.6$ ) years. Over $40 \%$ of participants were disabled $(43 \%)$ and/or obese $(44 \%$, mean $\mathrm{BMI}=33.4 \pm 31.5)$, and over $55 \%$ reported no exercise during the last month. Nearly half did not consume alcohol within the past year (46.8\%).

Health characteristics are displayed in Table 2 . As expected, the majority of study participants were experiencing chronic pain (93.4\%). Average GPS scores were high (mean=50.9 \pm 20.6 ), with $44 \%$ of participants reporting opioid use for pain management. The mean number of pain syndromes/conditions was $2.3(\mathrm{SD}=1.6)$, and prevalence of comorbidity was high, with $93 \%$ reporting at least 2 health conditions $($ mean $=5.4 \pm 3.1)$.

\section{Herbal Use}

Table 3 displays frequencies for the use of CHAs, including herbal use. Eight percent of participants $(n=24)$ used herbs for pain, with the majority using herbs for 6 months or more (Maintenance stage) (4.3\% of sample). Nineteen specific herbs used for pain management were reported; of these, the most commonly reported were ginger (41.7\%), lavender (33.3\%), peppermint (29.2\%), black cohosh (16.7\%), marijuana (12.5\%), and turmeric/curcumin (12.5\%). The mean age for those using herbs was $49.5(\mathrm{SD}=12.2)$ years, with nearly half of herbal users reporting at least a Bachelor's degree $(45.8 \%)$. Those using herbs had a mean of 6.1 health conditions ( $\mathrm{SD}=3.5)$ and a SF-GPS score of $56.9(\mathrm{SD}=18.2)$. Eighty-four percent of herbal users were using or had used prescriptions for pain; $63.2 \%$ of herbal users had used opioids (not shown). Nearly all herbal users were also using other CHAs (95.8\%), with the highest proportion using other dietary supplements (91.7\%). Over half of herbal users also reported using Acupuncture, Manipulative treatments, and/or Movement therapies (60.9\%), as well as Mind - Body practices $(56.5 \%)$.

\section{Relation of herbal use to demographic, lifestyle, and health factors (including other CHAs). Age} showed a marginally significant inverse association with herbal use which persisted after adjustment for 
education (adjusted odds ratio $(\mathrm{AOR})=0.97,95 \%$ confidence interval $(\mathrm{CI})$ 0.94, 1.01). After adjustment for age, those with the highest education level were approximately five times as likely to use herbs than those with the lowest education level (AOR $=4.94$, CI 1.59, 15.29) (Table 4). While herbal use was significantly and positively related to household income in the unadjusted model (OR $=1.59$, CI 1.07, 2.37 per $\$ 25,000$-unit income increase), this association was eliminated after adjustment for age and education $(\mathrm{AOR}=1.25$, CI $0.81,1.93)$. There were no significant differences in herbal use by other demographic or lifestyle factors.

Although there was no difference in herbal use by prescription medication, those using CHAs other than herbs were 11.5 times more likely to concomitantly use herbs compared to those not using CHAs (AOR = 11.46, CI 1.49, 87.93); specifically, in order of decreasing magnitude, those more likely to consume herbs included those using: Dietary supplements ( $\mathrm{OR}=12.26, \mathrm{CI} 2.82,53.38)$, Acupuncture, manipulative treatments, massage, and/or movement therapies $(\mathrm{OR}=5.15, \mathrm{CI} 2.11,12.60)$, followed by Mind-body practices $(\mathrm{OR}=3.83$, CI 1.59, 9.23). Adjustment for age and education only modestly attenuated these associations (Dietary supplements AOR = 10.25, CI 2.31, 45.47; Acupuncture, manipulative treatments, massage, and/or movement therapies AOR = 3.99, CI 1.55, 10.23; and, MindBody practices AOR $=2.44$, CI 0.94, 6.30). Short-term ( $<6$ months) dietary supplement users were 4.7 times more likely to use herbs than those not using dietary products for under 6 months (AOR $=4.46, \mathrm{CI}$ 1.76, 11.32); long-term ( $\geq 6$ months) dietary supplement use was not associated with concomitant herbal use $(\mathrm{AOR}=1.83$, CI 0.75, 4.49) (not shown). Additionally, short-term users of Acupuncture, manipulative treatments, massage, and/or movement therapies were 3 times as likely to use herbs (AOR = 2.97, CI, 1.09, 8.07), although long-term use of these CHAs was not associated with concomitant herbal use ( $\mathrm{AOR}=2.38$, CI 0.84, 6.70) (not shown). Conversely, longer-term users of Mind-Body practices were over 3 times more likely to use herbs than those not using mind and body practices long-term (AOR $=3.21$, CI 1.23, 8.37), while no significant association existed between short-term use of Mind-Body practices with herbal use (AOR $=0.50$, CI 0.10, 2.45) (not shown).

\section{Other CHA use}

CHAs other than herbs were used by the majority of survey participants $(58.8 \%, \mathrm{n}=177)$, with $47 \%$ of survey participants in the long-term/Maintenance ( $\geq 6$ months) stage of use; an additional $32 \%$ of participants were in the Contemplation or Preparation stage (i.e., had an intention to use within 6 months). Most commonly reported CHAs used were dietary supplements (53.6\% of participants), followed by Mind -Body practices (28.9\%) and Acupuncture, manipulative treatments, massage, and/or movement therapies $(28.1 \%)$. 
Relation of CHA use (excluding herbs) to demographic, lifestyle, and health factors. Relative to participants with a high school diploma or less, those with higher education levels were significantly more likely to use CHAs other than herbs than those with the lowest education levels (Crude OR's for $\geq$ Bachelor's degree and some college/Associate's degree/tech training $=2.55$ (CI 1.21, 5.40) and 2.11 (CI 1.17, 3.79), respectively); adjustment for age, exercise, health conditions, and GPS strengthened these associations (AOR for the highest vs lowest education level $=2.68$, CI 1.13, 6.39). CHA use was significantly and inversely associated with age $(\mathrm{OR}=0.98$, CI 0.96, 0.99) in the unadjusted model, although adjustment for education and exercise eliminated this association (AOR $=0.99$, CI 0.96, 1.01). (Table 5).

Exercise was strongly associated with CHA use in both crude and adjusted models, with a $14 \%$ increase in the odds of CHA use per 30-minute increase in exercise per week, after adjustment for age, education, GPS, and health conditions (AOR $=1.14$, CI 1.01, 1.29). Relative to those reporting no exercise, participants indicating engagement in physical activity were over twice as likely to use CHAs (fully adjusted OR =2.34, CI 1.29, 4.22). Reported SF-GPS, which includes pain severity, was also positively related to CHA use (OR adjusted for age, education, exercise, and health conditions=1.02 (CI $1.00,1,03)$ per unit GPS increase).

Sensitivity Analyses. Missing data was unrelated to specific patient characteristics, suggesting that missing data likely occurred due to inability to complete the survey while with a physician, or the wording/layout of the CHAPS, rather than a patient misunderstanding of what specific CHAs were. In addition, risk estimates for herbal/CHA use calculated using imputed data did not differ appreciably on any variable from those derived using the original CHAPS data set, suggesting that missing data are unlikely to account for our findings. Further, exclusion of participants in the pilot phase did not appreciably alter our findings.

Relation of specific CHA groups (excluding herbs) to demographic, lifestyle, and health factors. We examined the relationships between all covariates and: dietary supplements (other than herbs) (Table 6); Mind-body practices (Table 7); and Acupuncture and other approaches (Table 8). After controlling for demographic, lifestyle, and health factors, education level remained significantly and positively associated with all CHA categories, with Mind-body practices showing the strongest relationship (AOR for Bachelor's degree or higher $=6.45$, CI 2.71, 15.37). In addition, exercise remained significantly associated with dietary supplements $(\mathrm{AOR}=2.42, \mathrm{CI}=1.41,4.14)$ and Mind-body practices $(\mathrm{AOR}=$ 2.31 , CI 1.22, 4.39) after adjustment for other covariates, while associations with age were largely eliminated (Mind and body practices AOR $=0.98$ (CI 0.95, 1.00); Acupuncture and other approaches 
$\mathrm{AOR}=0.98(\mathrm{CI} 0.96,1.01))$. Those who were morbidly obese $(\mathrm{BMI} \geq 35)$ were $70 \%$ less likely to use Acupuncture, manipulative treatments, massage, and/or movement therapies relative to normal or underweight individuals ( $\mathrm{AOR}=0.32$, CI 0.12, 0.82) (Table 8); likewise, morbid obesity showed an inverse, although more modest association with use of dietary supplements other than herbs $(\mathrm{AOR}=0.40$, CI 0.16, 1.02) (Table 6).

Concomitant use of other CHAs was significantly and positively associated with all CHA categories; these associations persisted after adjustment for demographic, lifestyle, and health factors, with AORs ranging from $2.5(1.2,5.2)$ for dietary supplements (Table 6) to $3.3(1.6,6.7)$ for Acupuncture (and other manipulative/bodywork/movement approaches) (Table 8). Dietary supplement use was positively related to the number of health conditions (OR for total number of health conditions adjusted for demographic and lifestyle factors $=1.1, \mathrm{CI} 1.01,1.22$ ) and specifically to pain syndromes and injuries (partially adjusted OR's $=1.25(\mathrm{CI} 1.04,1.51)$ and 1.52 (CI 0.99, 2.34), respectively); these associations were modestly attenuated by the addition of SF-GPS to the model (Table 6).

\section{Discussion}

This was the first study to examine correlates of herbal use and other CHAs specifically used for pain in a chronic pain population, and among one of the first to examine correlates of herbal use and other CHAs in an Appalachian population, while controlling for a variety of potential confounders. To our knowledge, correlates of overall herbal use have only been assessed in the general population; unadjusted bivariate comparisons are seen more often in the literature, and many do not separate herbs from other natural products.

A wide variety of herbs were reported as having been used for pain management, some of which have at least preliminary evidence of benefit for pain, and/or have been anecdotally used for pain symptoms in Appalachian herbal texts and qualitative literature. Herbal users (and those using all other CHAs) had higher education levels, and were significantly more likely to concomitantly use a variety of CHAs; the strongest association was found between herbal use and short-term use of other dietary supplements. Other CHAs were used by almost $60 \%$ of participants; dietary supplements (excluding herbs) were the most commonly used CHA, followed by an approximately equal proportion of those using either Acupuncture (including manipulative or movement therapies, and/or massage), or, Mind and body practices. Additionally, CHA use was marginally associated with increased SF-GPS scores. Exercise was associated with the use of all individual CHAs, with the exception of herbal use. The $8 \%$ prevalence of herbal use in our study was higher than that reported in a recent study of primary care 
patients using opioids for pain management (93) and another U.S. study of chronic pain patients (95), but substantially lower than that reported in prior European (193-195). However, these differences may be due to the differing classification of our outcome of herbal use specifically for pain, rather than herbs used for a variety of purposes. The lower rates of herbal use reported in our study population may reflect the modest decline in the national use of herbs over the last decade (125), and/or differences in methodology, time period, and/or study population. For example, in their cross-sectional study of 463 chronic pain primary care patients sampled from multiple U.S. academic medical centers, Rosenberg et al. used an open-ended item administered with a personal interview format to assess herbal use; this may have allowed a broader capture of herbs and herbal preparations in their study (35). Two of the three European investigations assessing use of herbs for pain conditions were conducted 3 decades ago; including two U.K investigations indicating $20(193,194)$ to 35\% $(194,195)$ of Rheumatoid Arthritis patients used herbal medicines; one more recent (1996) survey study in another U.K. population suggested high prevalence of herbal use in FMS patients $(194,196,197)$, although the authors did not distinguish herbal from other dietary supplements, rendering comparisons with our findings challenging. Only two prevalence studies have assessed herbal use in non-pain Appalachian populations, including a 2003 prevalence study on 578 WV pregnant women which showed that $45.2 \%$ used herbs (184); however, this included soft drinks, cocoa butter for external use, and other products not counted as herbs in our study, while in 1999-2004, 19.3\% of 2523 rural NC adults over 45 years of age used herbs and other natural products, with garlic (4.9\%) and cayenne (1.8\%) the most widely reported (141).

In contrast, the reported prevalence of CHA use other than herbs was higher in our sample $(58.8 \%)$ than that reported in prior studies of U.S. chronic pain populations (93). For example, in a large sample of Wisconsin primary care patients using opioids for chronic pain, participants reported lower usage of several CHAs; the most striking contrast was the difference between supplement use in our sample (53.6\%) compared to that of the Wisconsin sample (6.8\% for herbs/supplements) $(93,95)$. Likewise, observed CHA use in our population also higher than that of reported by a sample of chronic pain primary care patients from academic medical centers in the US, although differences were more modest (58.8\% vs. $52 \%)(95)$.

Our preliminary systematic review on herbal prevalence among the general U.S. population (Appendix C) showed the majority of large cross-sectional studies frequently lumped herbs and dietary supplements together, resulting in uncertainty regarding true herbal prevalence rates; this was often due to misclassification of herbal use with other natural products (such as fish oil, etc.) in national surveys (primarily, the NHIS, which was used for a majority of these studies). Many studies assessing potential correlates of herbal use have used inconsistent definitions as well, often combining herbs with other dietary supplements $(141,143,144,146,147,155,156,159,161,165,344)$ or other approaches $(139$, 
145, 152, 154); few have kept the outcome herbal use separate from other CHAs $(138,157,160,162$, 345); our study separately classified herbal products from other dietary supplements.

Two herbs used in the Appalachian region and for which both evidence of anti-inflammatory and pain management exist were also reported in our sample; these were Ginger (223, 232, 233, 257-261) and Peppermint $(223,234,235,243)$ (41.7\% and 29.2\% of herbal users, respectively). Although not for pain, $18 \%$ of $578 \mathrm{WV}$ pregnant women used peppermint in a 2003 study (184). Lavender has at least preliminary evidence of efficacy for pain management (346-348), and anti-inflammatory (272) effects as well; our study is the first to report its use in an Appalachian sample. Herbs used most often by a U.S./Canadian sample of 434 with Fibromyalgia syndrome (FMS) were Ginkgo (3.7\%), Garlic (3.0\%), Ginseng (3.0\%), Echinacea (2.5\%), Evening Primrose oil (2.3\%), and Valerian (2.3\%) (197). With the exception of Ginkgo and Ginger, these were not reported by our participants; however, this was due to a difference in the assessment of herbal use for management of pain (in our sample) vs. herbal use for a variety of conditions.

Compared with nonusers, CHA users in the general population tend to be women, white, middle aged, better-educated, and of higher income (90, 106, 110-116). CHA use was not associated with female gender or race, and a borderline association between CHA use and (younger) age was eliminated when controlling for education, exercise, SF-GPS, and health conditions. Similarly, a study of 463 primary care patients with chronic pain also found no association between female gender, race, and age, after controlling for a variety of factors. It is plausible the demographic makeup and likelihood of CHA use differs in populations experiencing pain, compared to populations with a greater variety of health characteristics. Other CHA use was significantly associated with higher levels of education in our sample of pain patients, particularly mind and body practices; herbal use was as well.

Both CHA and herbal use in our sample were significantly and positively associated with higher education levels, consistent with previous studies, including two large 2002 studies which found those with a higher education were more likely to use CHAs $(95,144)$ and natural products $(144)$.After adjustment for other factors, income was not associated with herbal use or other CHAs, unlike a study of over 900 chronic pain patients using opioids (93). Although an income effect (349) may influence CHA use (i.e., higher-wage individuals are more likely to invest in health because they perceive higher benefits associated with improved health, including an increase in productive days and less sickness-related income loss), Bhargava et al. recently proposed that a substitution effect may also be present among those with higher incomes (i.e., higher wages may also increase the opportunity cost of time used for producing health, thus discouraging time spent on health care) (116). This may, perhaps, apply to CHAs use among those with higher incomes in pain populations; alternately, the impact of pain may influence individuals (regardless of income) to try approaches outside of or alongside conventional medicine. However, neither of our outcomes was associated the SF-GPS subscale, which incorporated pain severity as a component, 
even after controlling for income and other factors; this contrasts a cross-sectional study which found an adjusted (age, gender, race, pain sites, and illicit drug use) association between CHA use and pain severity without the use of the SF-GPS (93).

Overall, CHA users had higher odds of exercise and a marginal inverse association with higher BMI, associations of BMI and exercise were particularly pronounced with dietary supplement and Acupuncture (and manipulative/movement approaches) users. These findings are consistent with those from a study using 2012 NHIS data which indicated a significant, positive association of exercise to use of Chiropractic, Massage, herbs, and yoga (116). Herbal and CHA use was not associated with specific health conditions (such as both neck/upper back and multiple sites of pain), in contrast to findings reported in previous studies, including a larger study also controlling for demographic, health, and lifestyle factors similar to ours (93). Neither CHA nor herbal use was associated with prescription drug use in the CHAPS, similar to a large sample of primary care patients with chronic pain using opioids (95).

In addition, CHA use differed by those using a proxy or personal interview format, while no difference was found for herbal use. Thus, there may be a possibility that those lacking skills to complete the survey on their own may have limitations, perhaps disability and/or cognitive impairment, preventing them from using CHAs which require either physical movement or frequent practitioner visits - however, this may not prevent them from engaging in self-administered CHAs such as herbal use. Only one study has compared differences between self- and practitioner-administered CHA use, for which disability/cognitive impairment factors were not assessed (116).

This the first study to assess correlates of both herbal use and other CHAs in an Appalachian pain patient population. We pilot-tested and employed a newly developed survey instrument, the CHAPS. Incorporating pain items adapted from a previously validated pain measurement tool (315), the CHAPS included comprehensive information on a range of factors and constructs, yet required only 12 minutes to complete, and may thus be appropriate for use in future population- and clinic-based survey studies of pain populations. Survey response rates were high and we exceeded our target sample size, despite limited resources and other challenges including clinic lockdown, suspension of physician licensure, and inclement weather. In addition, our high reliability estimates for the SF-GPS also support further use of this measurement tool in pain populations.

Despite these strengths, there were also some limitations. Due to limited data regarding the use of CHAs in an Appalachian population with pain, we powered our study based on herbal prevalence rates reported in the literature for the general population (18\%); given that CHA use is known to be further elevated in chronic pain populations, we assumed that herbal use would be at least as high in our sample of Appalachian chronic pain patients. However, due to the lower than expected rates of herbal use in survey participants, we did not have sufficient power to examine associations by specific Stages of Change regarding herbal use, or to determine with confidence the prevalence of use of specific herbs. 
Many of our confidence intervals were wide, suggesting estimates should be interpreted with caution. Notably, some factors had relatively high rates of missing data, including the outcome herbal use (18.3\%), BMI (11.6\%), alcohol use (11\%), and income (12.3\%). However, we used multiple imputation procedures to account for missing data in order to obtain appropriate estimates of uncertainty associated with missing variables, assess for and minimize potential bias, and maximize the use of available information in our CHAPS dataset,; notably, estimates using imputed data did not differ appreciably from those derived from the original data, suggesting that missing data are unlikely to account for our findings. As there were no systematic patterns associated with missing data, and risk estimates did not appreciably change, we have confidence that estimates obtained from our data provided reasonably reliable measurements regarding the correlates of CHAs, including herbal use, in northern WV pain patients.

As in many studies using self-report data, there is a possibility of recall bias. While the misclassification of outcomes was unlikely, specific herbs or prescriptions listed in open-ended items may have suffered from misclassification bias, potentially resulting in underascertainment. For example, we overheard a proxy interviewer ask a respondent, "Do you want to list your prescriptions?" to which the respondent replied, "No, it would take too long.". Additionally, we did not incorporate specific questions about marijuana due to concerns regarding the sensitivity of this issue. Nonetheless, marijuana use for pain was still reported by $12.5 \%$ of herbal users in our open-ended item, a figure that likely substantially underestimates true prevalence.

Selection bias may have been present since this study utilized a convenience sample. However, our consecutive sampling approach minimized this possibility; in addition, response rates were very high, reducing the likelihood of non-response bias. Researchers wishing to implement the CHAPS or similar surveys should explore barriers to survey implementation present at specific clinics, including the physical space made available to them; one clinic was gracious enough to offer our coordinator a workstation behind their check-in window during data collection days, and although all patients were solicited for participation, this site had the lowest overall response rate (84.1\%) of all clinics.

Additionally, much remains unknown about the health behavior habits of opioid users with regard to CHA use. Our measurement of CHAs, particularly herbal use, was hindered by a high proportion of missing responses among those using opioids, which comprised nearly half of our population, potentially leading to an underestimate for herbal use. Other issues surrounding the use of our survey instrument include a lack of data on specific reasoning to use herbs and other CHAs. This was the result of our decision to keep the CHAPS as brief as possible; however, future studies may wish to incorporate more contextual elements of herbal use and CHAs, including specific reasons for use, concomitant use of medications, spirituality and/or prayer, access to CHAs, and disclosure of CHA use to physicians. 
Additional considerations should be made for the capture of information in future iterations of the CHAPS, or for similar surveys. These include additional questions regarding e-cigarettes and other forms of tobacco, capture of previous use of CHAs beyond the 6-month time point assessed in our study, and reformatting CHA questions with equally-spaced bins on multiple pages with adequate visual cues and space for nested efficacy questions.

\section{Conclusion}

Our study was the first to assess concomitant CHA use in a pain population and to document specific herbs used within this population. Although the majority of patients in our sample did not use herbs for pain, their use was reported $8 \%$ of the time and through the use of 19 unique herbs, and was strongly and positively associated with the use of other CHAs. The majority of our sample used at least one CHA for pain. Investigation of factors associated with herbal medicine, other CHAs, and their concomitant use in pain populations will help inform physicians concerned about potential interactions. Additional studies are needed to further explore the patterns and determinants of herbal and other CHA use in chronic pain populations, to assess perceived efficacy of these approaches, and to lay the foundation for future clinical efficacy studies on herbal and other CHA approaches for managing chronic pain.

\section{CHAPTER 5: Discussion}

\section{Summary of Key Findings}

Inflammation is often a component of chronic pain, yet its potential role in fibromyalgia syndrome (FMS) remains inconclusive. FMS is a complex chronic pain condition affecting $2 \%$ of the population; management is challenging and treatment options remain limited. Many herbs contain antiinflammatory properties, and herbs indicated for analgesia and rheumatic conditions have traditionally been used in Appalachia. Despite the popularity of herbs in the US, determinants and patterns of herbal use in FMS and other chronic pain populations have not been well studied, particularly in Appalachia, where prevalence of chronic pain and related comorbid conditions is high. Likewise, prevalence, patterns and correlates of other CHA use remain little studied in Appalachian chronic pain populations, or in adults with certain pain conditions, including FMS. This series of 3 studies are designed to help fill these knowledge gaps; the overall objectives of these investigations were to investigate: 1) the association of 
FMS to a marker of inflammation, serum C-reactive protein (CRP) in a large Appalachian population; 2) the demographic, lifestyle, and health-related correlates of herbs and other complementary health approaches (CHAs) used for pain in a sample of WV chronic pain patients, using a newly developed survey instrument; and, 3) the relation of herbal supplement use to FMS in two nationally representative samples of U.S. adults (NHIS 2007 and 2012), as well as trends in patterns of herbal use over time.

Although we found herbal use was associated with concurrent use of other CHAs in WV pain patients, it was not associated with pain level, the use of prescription medications, or the number of pain conditions. Results of these studies suggest the following 1) FMS is positively associated with serum CRP; this association was largely explained by obesity and comorbidity, suggesting the role of inflammation in FMS may be complex 2) CHA use in chronic pain patients in northern WV is high, although reported use of herbs for pain appears low. Herbal use for pain was strongly associated with use of other CHAs, but was unrelated to pain severity or use of prescription medications. 3) Use of herbs and other CHAs is strongly and positively associated with FMS diagnosis in U.S adults.

\section{Strengths of the Dissertation}

All studies had high response rates and population-based designs. Sample sizes for two of the three studies were large. The C8 Health Project, from which we drew data for Chapter 2, was the largest comprehensive community study conducted to date in the Appalachian region; Chapter 3 was conducted using two nationally representative samples of US adults. Chapter 4, the CHAPS, was the first to assess correlates of CHAs and herbal use in an Appalachian pain population and to employ a newly developed comprehensive survey instrument for measuring herbal and other CHA use, pain severity, and a wide range of demographic, lifestyle, and health-related factors (see below). With the exception of analyses regarding correlates of herbal use in the CHAPS, we were also able to control for a broad array of potential confounders, including health conditions, which diminished the possibility of unmeasured confounding. We were also able to assess the influence of a variety of potential mediators and/or effect modifiers in our analyses.

In Chapter 4, we employed our newly developed survey instrument, the CHAPS, in over 300 pain patients, utilizing independent investigators to conduct quality assessment, and incorporating pain items adapted from a previously-validated pain measurement tool (315). Although allowing for capture of information on a wide array of health-related information, the CHAPS required only 10-12 minutes to complete; reliability of the CHAPS was also excellent overall, indicating high internal reliability estimates and further supporting the use of this instrument for observational research in chronic pain populations. We exceeded our target sample size, even though we faced significant obstacles to data collection including clinic lockdown, inclement mountain weather, and temporary withdrawal of physician licensure which limited our access to patients. In addition, we conducted a sensitivity analysis 
using imputation procedures to verify our estimates against those without missing data, which enabled us to interpret our findings with confidence.

\section{Limitations of the Dissertation}

Although our studies had many strengths, there were several limitations. Most important conclusions regarding causality cannot be drawn due to the lack of a temporal component in our crosssectional study designs. Two of our studies targeted populations of predominantly white Appalachian adults, potentially limiting the generalizability of findings to other populations. Moreover, our original study in a sample of WV chronic pain patients was underpowered to assess herbal use based on the TTM Stages of Change as originally envisioned. Given the often poor recognition of FMS in the medical community, particularly prior to 2010 , misclassification is also possible and may have led to underascertainment. Nearly all health conditions, and all herbal/CHA use measurements in our studies were based on self-report, possibly leading to response or misclassification bias.

Additionally, neither NHIS dataset captured the use of herbal products other than supplements, likely leading to underestimates of herbal use. Likewise, many of the CHAs were only assessed in the NHIS if they were administered by a practitioner, likely resulting in underestimates, which may have impacted our ability to measure concurrent CHA and herbal use accurately. Additionally, we were unable to incorporate an additional year of the NHIS (2002) due to a lack of specific information regarding FMS diagnosis.

\section{Potential Public Health Implications and Future Recommendations}

FMS, a disease with largely unknown etiology, has higher odds of comorbid conditions compared to non-FMS populations; this includes inflammatory-mediated diseases such as autoimmune disease (22). However, the potential shared role of inflammatory markers between FMS and specific comorbidities remains largely unexplored by population studies. Because we were only able to assess the role of CRP as an overall measure of systemic inflammation, however, we remain unaware of specific dynamics regarding inflammatory response, or interplay, between other cytokines (and related markers) in those with FMS, in the presence of obesity and comorbidities such as autoimmune disease.

Although it is possible inflammation is not associated with FMS, given the results of our first study, further large, population-based research is still needed to replicate our findings while assessing for a variety of inflammatory markers while controlling for a variety of factors and assessing potential mediators as we did; based on established knowledge of the anti-inflammatory effects of some herbs $(123,223,225-227,233,241-268,270-272)$, and, given FMS was positively associated with herbal use in 
our second study, future research should also compare a variety of inflammatory measures among those using herbs (ideally, with known anti-inflammatory benefit) by FMS status; based on sensitivity analysis results from our second study as well, the role of comorbidities in research on herbal use in FMS populations should be further explored.

As a result of our second study, we have a better understanding of the patterns of herbal use prior to and following the implementation of updated FMS diagnostic criteria and increased availability of FDA-approved treatments for FMS. It remains possible that both the increase in FMS diagnoses and the decrease in odds of herbal use for FMS over time are also in part perhaps the result of corporate promotion for FMS drugs, increased off-label use of other drugs, and, opioid use for FMS. Additional research should continue to monitor FMS trends, in addition to herbal use trends over time for a variety of diseases; all trend studies should continue to consider changes in diagnostic criteria as well as the changing availability of treatments for respective diseases.

Whether opioids can be reduced by introducing herbal products remains to be examined. Our second and third studies offer information regarding the patterns and correlates of complementary health practices in specific chronic pain populations in WV and nationally. Although efficacy studies for such approaches, including herbs, are largely lacking, our research may encourage a more open dialogue between physicians and patients about potential opioid alternatives at a time when there is a public health epidemic of opioid misuse and abuse (86). However, studies regarding perceived or demonstrated efficacy of herbs for FMS and other pain syndromes remain sparse; unfortunately, we were unable to adequately measure efficacy on a number of CHAs due to low sample size. Nonetheless, clinical studies should ideally examine aspects of CHA and herbal efficacy in pain populations rather than observational research; with the inclusion of a larger sample size, the CHAPS will be a useful tool in identification of potential herbs and other CHAs of interest. Future observational and clinical studies should, however, capture concurrent dietary supplements and medication intakes, including opioids, to enable assessment of potential herb-drug interactions in populations with disease.

Neither CHAs nor herbal use were strongly associated with the SF-GPS subscale in our third study; however, $8 \%$ of pain patients still reported using herbs specifically for pain. Due to lack of a standard definition for herbal products, true herbal prevalence rates for pain are unknown. Moving toward an international consensus regarding a widely-used definition for herbal products will only improve the quality of research by enabling a greater degree of comparison between studies. Other CHAs were used by $58.8 \%$ of participants in our sample; again, the comparability of our results to other studies is greatly hampered by the lack of standardized CHA definitions.

Herbs reported as used for pain management in our third study included ginger and peppermint, which have at least preliminary evidence of benefit for pain and/or inflammation, and have been anecdotally used for pain in Appalachian herbal texts and qualitative literature (223, 232-235). Our study was the first 
to report pain patient use of lavender, an herb for which some evidence of anti-inflammatory mechanism exists (272); lavender was used specifically for pain in our sample. While ethnobotanical studies are important in documenting herbal preparations in a variety of populations for health purposes, our epidemiological approach to quantifying the use of herbs is also useful; this approach lays the foundation for understanding differences regarding a variety of factors associated with herbal use in subpopulations, while enabling our ability to draw estimates for measures of association. Replication of the CHAPS in a larger population may yield additional information about the correlates of specific herbs; these future studies may inform differences in herbal use by a variety of demographic, lifestyle, and health factors, to lay the foundation for clinical studies or when clinical studies are not feasible.

\section{Conclusion}

Our studies, collectively, demonstrate a need for further clarification regarding the relationships between herbal use, FMS, and inflammation. Our main findings suggest that: inflammation in FMS may be partially accounted for by factors including BMI, comorbidities, and other factors; those with FMS are significantly more likely to use herbs; and, although herbal use (specifically) for pain may be strongly associated with concurrent use of other CHAs for pain, their use may not be associated with pain level, but other factors. Future observational and longitudinal studies must replicate our studies with specific improvements: correct classification of herbal use as plant-based products consumed in a variety of formats; multiple, concurrent, inflammatory marker measurement; ascertainment of FMS diagnosis timing; and, for CHAPS, larger samples to obtain more precise estimates regarding correlates of herbal use for pain. 


\section{References}

\section{Chapter 1}

1. Myers KA, Bello-Espinosa LE, Kherani A, Wei XC, Innes AM. TUBA1A Mutation Associated With Eye Abnormalities in Addition to Brain Malformation. Pediatr Neurol. 2015. doi: 10.1016/j.pediatrneurol.2015.07.004. PubMed PMID: 26294046.

2. Cauffield JS. The Psychoscoial aspects of complementary and alternative medicine. Pharmacotherapy. 2000;20(11):1289-94.

3. Manchikanti L, Singh V, Datta S, Cohen SP, Hirsch JA. American Society of Interventional Pain Physicians. Comprehensive review of epidemiology, scope, and impact of spinal pain. Pain Physician. 2009;12:E35-70.

4. Andersson HI. The epidemiology of chronic pain in a Swedish rural area. Qual Life Res. 1994;3:s19-s26.

5. Brattberg G, Thorslund M, Wikman A. The prevalence of pain in a general population. The results of a postal survey in a county of Sweden. Pain. 1989;37:215-22.

6. Wijnhoven HA, de Vet HC, Picavet HS. Explaining sex differences in chronoc musculoskeletal pain in a general population. Pain. 2006;124:158-66.

7. White KP, Harth M. Classification, epidemiology, and natural history of fibromyalgia. Current pain and headache reports. 2001;5(4):320-9. doi: 10.1007/s11916-001-0021-2.

8. Walitt B, Nahin RL, Katz RS, Bergman MJ, Wolfe F. The Prevalence and Characteristics of Fibromyalgia in the 2012 National Health Interview Survey. PLoS One. 2015;10(9):e0138024. doi: 10.1371/journal.pone.0138024. PubMed PMID: 26379048.

9. Clauw DJ. Fibromyalgia and related conditions. Mayo Clin Proc. 2015;90(5):680-92. doi: 10.1016/j.mayocp.2015.03.014. PubMed PMID: 25939940.

10. Baker K, Barkhuizen A. Pharmacologic treatment of fibromyalgia. Curr Pain Headache Rep. 2005;9(5):301-6. PubMed PMID: 16157056.

11. Administration FaD. Living with Fibromyalgia, Drugs Approved to Manage Pain Food and Drug Administration: Food and Drug Administration; 2013 [cited 2015 April 10]. Consumer Health Information]. Available from: http://www.fda.gov/downloads/ForConsumers/ConsumerUpdates/UCM107805.pdf.

12. Buskila D, Atzeni F, Sarzi-Puttini P. Etiology of fibromyalgia: The possible role of infection and vaccination. Special Issue on Infections Rheumatism and Autoimmunity. 2008;8(1):41-3. doi: http://dx.doi.org.www.libproxy.wvu.edu/10.1016/j.autrev.2008.07.023.

13. NIH. Questions and Answers about Fibromyalgia 2014 [cited 2014 July]. Available from: http://www.niams.nih.gov/Health Info/Fibromyalgia/default.asp.

14. Bennett RM, Jones J, Turk DC, Russell IJ, Matallana L. An internet survey of 2,596 people with fibromyalgia. BMC musculoskeletal disorders. 2007;8:27. doi: 10.1186/1471-2474-8-27. PubMed PMID: 17349056; PubMed Central PMCID: PMCPMC1829161.

15. Arnold LM, Hudson JI, Hess EV, Ware AE, Fritz DA, Auchenbach MB, et al. Family study of fibromyalgia. Arthritis and rheumatism. 2004;50(3):944-52. Epub 2004/03/17. doi: 10.1002/art.20042. PubMed PMID: 15022338.

16. Clauw DJ, Crofford LJ. Chronic widespread pain and fibromyalgia: what we know, and what we need to know. Chronic Generalised Musculoskeletal Pain. 2003;17(4):685-701. doi: http://dx.doi.org.www.libproxy.wvu.edu/10.1016/S1521-6942(03)00035-4.

17. Wolfe F, Clauw Dj Fau - Fitzcharles M-A, Fitzcharles Ma Fau - Goldenberg DL, Goldenberg D1 
Fau - Hauser W, Hauser W Fau - Katz RS, Katz Rs Fau - Mease P, et al. Fibromyalgia criteria and severity scales for clinical and epidemiological studies: a modification of the ACR Preliminary Diagnostic Criteria for Fibromyalgia.

18. Fok E, Sandeman SR, Guildford AL, Martin YH. The Use of an IL-1 Receptor Antagonist Peptide to Control Inflammation in the Treatment of Corneal Limbal Epithelial Stem Cell Deficiency. Biomed Res Int. 2015;2015:516318. doi: 10.1155/2015/516318. PubMed PMID: 25705668; PubMed Central PMCID: PMC4330955.

19. Bjersing JL, Bokarewa MI, Mannerkorpi K. Profile of circulating microRNAs in fibromyalgia and their relation to symptom severity: an exploratory study. Rheumatol Int. 2015;35(4):635-42. doi: 10.1007/s00296-014-3139-3. PubMed PMID: 25261961.

20. Bjersing JL, Lundborg C, Bokarewa MI, Mannerkorpi K. Profile of cerebrospinal microRNAs in fibromyalgia. PLoS One. 2013;8(10):e78762. doi: 10.1371/journal.pone.0078762. PubMed PMID: 24205312; PubMed Central PMCID: PMCPMC3808359.

21. Ursini F, Naty S, Grembiale R. Fibromyalgia and obesity: the hidden link. Rheumatol Int. 2011;31(11):1403-8. doi: 10.1007/s00296-011-1885-z.

22. Giacomelli C, Talarico R, Bombardieri S, Bazzichi L. The interaction between autoimmune diseases and fibromyalgia: risk, disease course and management. Expert review of clinical immunology. 2013;9(11):1069-76. doi: 10.1586/1744666X.2013.849440. PubMed PMID: 24168413.

23. Staud R. Evidence for shared pain mechanisms in osteoarthritis, low back pain, and fibromyalgia. Current rheumatology reports. 2011;13(6):513-20. doi: 10.1007/s11926-011-0206-6. PubMed PMID: 21833699.

24. Wolfe F, Michaud K, Li T, Katz RS. Chronic conditions and health problems in rheumatic diseases: comparisons with rheumatoid arthritis, noninflammatory rheumatic disorders, systemic lupus erythematosus, and fibromyalgia. The Journal of rheumatology. 2010;37(2):305-15. doi: 10.3899/jrheum.090781. PubMed PMID: 20080915.

25. Ozgocmen S, Cimen OB, Ardicoglu O. Relationship between chest expansion and respiratory muscle strength in patients with primary fibromyalgia. Clinical rheumatology. 2002;21(1):19-22. PubMed PMID: 11954878.

26. Yang TY, Chen CS, Lin CL, Lin WM, Kuo CN, Kao CH. Risk for irritable bowel syndrome in fibromyalgia patients: a national database study. Medicine. 2015;94(10):e616. doi: 10.1097/MD.0000000000000616. PubMed PMID: 25761187.

27. Yanmaz MN, Mert M, Korkmaz M. The prevalence of fibromyalgia syndrome in a group of patients with diabetes mellitus. Rheumatol Int. 2012;32(4):871-4. doi: 10.1007/s00296-01016188. PubMed PMID: 21221595.

28. Michaud K, Wolfe F. The association of rheumatoid arthritis and its treatment with sinus disease. The Journal of rheumatology. 2006;33(12):2412-5. PubMed PMID: 17143978.

29. Tietjen GE, Brandes JL, Peterlin BL, Eloff A, Dafer RM, Stein MR, et al. Allodynia in migraine: association with comorbid pain conditions. Headache. 2009;49(9):1333-44. doi: 10.1111/j.15264610.2009.01521.x. PubMed PMID: 19788473.

30. Devor M. - Neuropathic pain: what do we do with all these theories? 2001;- 45(- 9):- 1127.

31. Lipnik-Stangelj M. Mediators of inflammation as targets for chronic pain treatment. Mediators of inflammation. 2013;2013:783235. doi: 10.1155/2013/783235. PubMed PMID: 24347834; PubMed Central PMCID: PMC3848385.

32. Watkins LR, Milligan ED, Maier SF. Glial proinflammatory cytokines mediate exaggerated pain states: implications for clinical pain. Advances in experimental medicine and biology. 2003;521:1-21. PubMed PMID: 12617561.

33. Zhang J-M, An J. Cytokines, Inflammation and Pain. International anesthesiology clinics. 2007;45(2):27-37. doi: 10.1097/AIA.0b013e318034194e. PubMed PMID: PMC2785020. 
34. FibroCenter. Fibromyalgia Pain Is Different: Pfizer; 2015 [cited 2015]. Available from: http://www.fibrocenter.com/fibromyalgia-pain.aspx.

35. Maes M, Libbrecht I, Van Hunsel F, et al. The immune-inflammatory pathophysiology of fibromyalgia: increased serum soluble gp130, the common signal transducer protein of various neurotropic cytokines. Psychoneuroendocrinology. 1999;24(4):371-83.

36. Wallace DJ L-IM, Hallegua D, Silverman S, Silver MH, Weisman MH. Cytokines play an aetiopathogenetic role in fibromyalgia: a hypothesis and pilot study. Rheumatology (Oxford). 2001;40(7):743-9.

37. Gur A KM, Erdogan S, Nas K, Cevik R, Sarac AJ. Regional cerebral flow and cytokines in young females with fibromyalgia. Clinical and experimental rheumatology JID - 8308521. 2002;20(6):753-60.

38. Gur A KM, Nas K, Remzi C, Denli A, Sarac J. Cytokines and depression in cases with fibromyalgia. Journal of Rheumatology. 2002;29(2):358-3561.

39. Hein G FS. Are advanced glycaton end-product-modified proteins of pathogentic importance in fibromyalgia. Rheumatology. 2002;41(10):1163-7.

40. Pache M OJ, Genth E, Mierau R, Kube T, Flammer J. Increased plasma endothilin-1 levels in fibromyalgia syndrome. Rheumatology. 2003;42(493-494).

41. Bagis S TL, Sahin G, et al. Free radicals and antioxidants in primary fibromyalgia: an oxidative stress disorder? Rheumatology international JID - 8206885.

42. Salemi S RJ, Wollina U, et al. Detection of interleukin 1 beta (IL-beta), IL0-6, and Tumor necrosis factor-alpha in skin of patients with fibromyalgia. Journal of Rheumatology. 2003;30(1):146-50.

43. Kadetoff DF, Lampa JF, Westman MF, Andersson MF, Kosek E. Evidence of central inflammation in fibromyalgia-increased cerebrospinal fluid interleukin-8 levels. 0328.

44. Xiao Y HW, Michalek JE, Russell IJ. Elevated serum high-sensitivity C-reactive protein levels in fibromyalgia syndrome patients correlate with body mass index, interleukin-6, interleukin-8, erythrocyte sedimentation rate. 2013.

45. Kaufmann IF, Schelling GF, Eisner CF, Fau RHP, Beyer AF, Krauseneck TF, et al. Decrease in adhesion molecules on polymorphonuclear leukocytes of patients with fibromyalgia. 0924.

46. Bazzichi LF, Rossi AF, Massimetti GF, Giannaccini GF, Giuliano T Fau DF, De Feo FF, et al. Cytokine patterns in fibromyalgia and their correlation with clinical manifestations. 0830.

47. Bazzichi L RA, Massimetti G, et al. Cytokine patterns in fibromyalgia and their correlation with clinical manifestations. Clin and Experim Rheum 2007;25(2):225-30.

48. Lund Haheim L, Nafstad P, Olsen I, Schwarze P R, KS. C-reactive protein variations for different chronic somatic disorders. 1001.

49. Rus A, Molina F, Gasso M, Camacho MV, Peinado MA, Moral ML. Nitric Oxide, Inflammation, Lipid Profile, and Cortisol in Normal- and Overweight Women With Fibromyalgia. Biological research for nursing. 2015. doi: 10.1177/1099800415591035. PubMed PMID: 26134428.

50. Amel Kashipaz M, Swinden D, Todd I, Powell R. Normal production of inflammatory cytokines in chronic fatigue and fibromyalgia syndromes determined by intracellular cytokine staining in short-term cultured blood mononuclear cells. Clinical and experimental immunology. 2003;132(2):360-5. PubMed PMID: 12699429; PubMed Central PMCID: PMCPMC1808704.

51. Afsar B, Burucu R. Urinary albumin, protein excretion and circadian blood pressure in patients with fibromyalgia. Rheumatol Int. 2013;33:2391-8. doi: 10.1007/s00296-013-2748-6.

52. Sherman KJ, Innes KE. Yoga for metabolic risk factors: much ado about nothing or new form of adjunctive care? Journal of diabetes and its complications. 2014;28(3):253-4. doi: 10.1016/j.jdiacomp.2014.03.001. PubMed PMID: 24642263.

53. Bernardy K, Klose P, J BA, HS CE, Häuser W. : Cognitive behavioural therapies for fibromyalgia. : Cochrane Database of Systematic Reviews. 
54. Andrew MR, Derry S, Aldington D, Cole P, J WP. : Amitriptyline for neuropathic pain and fibromyalgia in adults. : Cochrane Database of Systematic Reviews.

55. L RB, L WS, Buchbinder R. : Neuromodulators for pain management in rheumatoid arthritis. : Cochrane Database of Systematic Reviews.

56. Andrew MR, J WP, Derry S, Toelle T, C RAS. : Gabapentin for chronic neuropathic pain and fibromyalgia in adults. : Cochrane Database of Systematic Reviews.

57. Roskell NS, Beard SM, Zhao Y, Le TK. A meta-analysis of pain response in the treatment of fibromyalgia. Pain practice : the official journal of World Institute of Pain. 2011;11(6):516-27. doi: 10.1111/j.1533-2500.2010.00441.x. PubMed PMID: 21199320.

58. PT LM, AC HR, J WP. : Duloxetine for treating painful neuropathy, chronic pain or fibromyalgia. : Cochrane Database of Systematic Reviews.

59. Häuser W, Urrútia G, Tort S, Üçeyler N, Walitt B. : Serotonin and noradrenaline reuptake inhibitors (SNRIs) for fibromyalgia syndrome. : Cochrane Database of Systematic Reviews.

60. Derry S, Gill D, Phillips T, Andrew MR. : Milnacipran for neuropathic pain and fibromyalgia in adults. : Cochrane Database of Systematic Reviews.

61. J WP, Derry S, Andrew MR, Aldington D, Cole P, C RAS, et al.: Antiepileptic drugs for neuropathic pain and fibromyalgia - an overview of Cochrane reviews. : Cochrane Database of Systematic Reviews.

62. Lanza FL, Chan FK, Quigley EM, Practice Parameters Committee of the American College of G. Guidelines for prevention of NSAID-related ulcer complications. The American journal of gastroenterology. 2009;104(3):728-38. doi: 10.1038/ajg.2009.115. PubMed PMID: 19240698.

63. Zhao Y, Sun P, Watson P, Mitchell B, Swindle R. Comparison of medication adherence and healthcare costs between duloxetine and pregabalin initiators among patients with fibromyalgia. Pain practice : the official journal of World Institute of Pain. 2011;11(3):204-16. doi: 10.1111/j.1533-2500.2010.00412.x. PubMed PMID: 20807351.

64. Busse JW, Ebrahim S, Connell G, Coomes EA, Bruno P, Malik K, et al. Systematic review and network meta-analysis of interventions for fibromyalgia: a protocol. Systematic reviews. 2013;2:18. doi: 10.1186/2046-4053-2-18. PubMed PMID: 23497523; PubMed Central PMCID: PMC3610251.

65. Noller V, Sprott H. Prospective epidemiological observations on the course of the disease in fibromyalgia patients. Journal of negative results in biomedicine. 2003;2:4. doi: 10.1186/14775751-2-4. PubMed PMID: 12969513; PubMed Central PMCID: PMC194775.

66. Reid S, Whooley D, Crayford T, Hotopf M. Medically unexplained symptoms--GPs' attitudes towards their cause and management. Family practice. 2001;18(5):519-23. PubMed PMID: 11604375.

67. Birse F, Derry S, Andrew MR. : Phenytoin for neuropathic pain and fibromyalgia in adults. : Cochrane Database of Systematic Reviews.

68. Corrigan R, Derry S, J WP, Andrew MR. : Clonazepam for neuropathic pain and fibromyalgia in adults. : Cochrane Database of Systematic Reviews.

69. J WP, Derry S, Andrew MR, A KE. : Carbamazepine for chronic neuropathic pain and fibromyalgia in adults. : Cochrane Database of Systematic Reviews.

70. J WP, Derry S, PT LM, Andrew MR. : Topiramate for neuropathic pain and fibromyalgia in adults. : Cochrane Database of Systematic Reviews.

71. Tort S, Urrútia G, Nishishinya María B, Walitt B. : Monoamine oxidase inhibitors (MAOIs) for fibromyalgia syndrome. : Cochrane Database of Systematic Reviews.

72. Gill D, Derry S, J WP, Andrew MR. : Valproic acid and sodium valproate for neuropathic pain and fibromyalgia in adults. : Cochrane Database of Systematic Reviews.

73. J WP, Derry S, Andrew MR. : Lamotrigine for chronic neuropathic pain and fibromyalgia in adults. : Cochrane Database of Systematic Reviews. 
74. Hearn L, Derry S, Andrew MR. : Lacosamide for neuropathic pain and fibromyalgia in adults. : Cochrane Database of Systematic Reviews.

75. Gaskell H, Andrew MR, Derry S, Stannard C. : Oxycodone for neuropathic pain and fibromyalgia in adults. : Cochrane Database of Systematic Reviews.

76. Rheumatology ACo. Patient Resources: Diseases and Conditions - Fibromyalgia: American College of Rheumatology; 2013 [cited 2015 April 10]. Available from: http://www.rheumatology.org/Practice/Clinical/Patients/Diseases_And_Conditions/Fibromyalgia/

77. Ernst E. Oxford handbook of complementary medicine. Oxford ; New York: Oxford University Press; 2008. xxiii, 424 p. p.

78. Schoonees A, Visser J, Musekiwa A, Volmink J. : Pycnogenol® (extract of French maritime pine bark) for the treatment of chronic disorders. : Cochrane Database of Systematic Reviews.

79. Plaganyi EE, van Putten I, Thebaud O, Hobday AJ, Innes J, Lim-Camacho L, et al. A quantitative metric to identify critical elements within seafood supply networks. PLoS One.

2014;9(3):e91833. doi: 10.1371/journal.pone.0091833. PubMed PMID: 24633147; PubMed Central PMCID: PMCPMC3954797.

80. Scheuermeyer FX, Wong H, Yu E, Boychuk B, Innes G, Grafstein E, et al. Development and validation of a prediction rule for early discharge of low-risk emergency department patients with potential ischemic chest pain. CJEM. 2014;16(2):106-19. PubMed PMID: 24626115.

81. Santamaria RM, Innes NP, Machiulskiene V, Evans DJ, Alkilzy M, Splieth CH. Acceptability of different caries management methods for primary molars in a RCT. Int J Paediatr Dent. 2015;25(1):9-17. doi: 10.1111/ipd.12097. PubMed PMID: 24602167.

82. Adams D, Wu T, Yang X, Tai S, Vohra S. : Traditional Chinese medicinal herbs for the treatment of idiopathic chronic fatigue and chronic fatigue syndrome. : Cochrane Database of Systematic Reviews.

83. Painter JT, Crofford LJ. Chronic opioid use in fibromyalgia syndrome: a clinical review. Journal of clinical rheumatology : practical reports on rheumatic \& musculoskeletal diseases. 2013;19(2):72-7. doi: 10.1097/RHU.0b013e3182863447. PubMed PMID: 23364665.

84. CDC. Drug-poisoning Deaths Involving Opioid Analgesics: United States, 1999-2011: CDC; 2014 [cited 2015 4/17]. Available from: http://www.cdc.gov/nchs/data/databriefs/db166.htm.

85. Williams DA, Clauw DJ. Understanding fibromyalgia: lessons from the broader pain research community. The journal of pain : official journal of the American Pain Society. 2009;10(8):77791. doi: 10.1016/j.jpain.2009.06.001. PubMed PMID: 19638325; PubMed Central PMCID: PMC2741022.

86. O'Neil M, Hannah KL. Understanding the cultures of Prescription Drug Abuse, Misuse, Addiction, and Diversion. WV Medical Journal. 2010;106(4):64-70.

87. Painter JT, Crofford LJ, Talbert J. Geographic variation of chronic opioid use in fibromyalgia. Clin Ther. 2013;35(3):303-11. doi: 10.1016/j.clinthera.2013.02.003. PubMed PMID: 23485077; PubMed Central PMCID: PMC4346177.

88. Rosenberg EI, Genao I, Chen I, Mechaber AJ, Wood JA, Faselis CJ, et al. Complementary and alternative medicine use by primary care patients with chronic pain. Pain Med. 2008;9(8):106572. doi: 10.1111/j.1526-4637.2008.00477.x. PubMed PMID: 18564996.

89. Astin J. Why patients use alternative medicine: Results of a national survey. JAMA. 1998;279(19):1548-53.

90. Eisenberg DM, Kessler RC, Foster C, Norlock FE, Calkins DR, Delbanco TL. Unconventional medicine in the United States: Prevalence, costs, and patterns of use. New England Journal of Medicine. 1993;328:246-52. 
91. Eisenberg DM, Kessler RC, Van Rompay MI, Kaptchuk TJ, Wilkey SA, Appel S, et al. Perceptions about complementary therapies relative to conventional therapies among adults who use both: Results from a national survey. Ann Intern Med. 2001;135:344-51.

92. Barret B, Marchand L, Scheder J, Appelbaum D, Plane MB, Blustein J, et al. What complementary and alternative medicine practitioners say about health and health care. Ann Fam Med. 2004;2:253-9.

93. Fleming $\mathrm{S}$ et al. CAM therapies among primary care patients using opioid therapy for chronic pain. BMC Complementary and Alternative Medicine. 2007;7(15).

94. Sherman K et al. Complementary and alternative medical therapies for chronic low back pain: what treatments are patients willing to try? BMC Complementary and Alternative Medicine. 2004;4(9).

95. Rosenberg E et al. Complementary and Alternative Medicine Use by Primary Care Patients with Chronic pain. Pain medicine (Malden, Mass) JID - 100894201. 2008;9(8):1065-72.

96. Druss BG, Rosenheck RA. Association between use of unconventional therapies and conventional medical services. JAMA. 1999;282(7):651-6. PubMed PMID: 10517718.

97. Rao JK, Mihaliak K, Kroenke K, et al. Use of complimentary therapies for arthrtitis among patients of rheumatologists. Ann Intern Med. 1999;131(6):409-16.

98. Brown CM. Use of Alternative therapies and their impact on compliance: perceptions of community pharmacists in Texas. J Am Pharm Assoc. 1998;38(5):603-8.

99. Berman BM, Singh BB, Hartnoll SM, Singh BK, Reilly D. Primary care physicians and complementary/alternative medicine: Training, attitudes and practice patterns. J Am Board Fam Pract. 1998;11:272-81.

100. Wagner PJ, Jester D, LeClair B, et al. Taking the edge off: why patients choose St. John's Wort. J Fam Pract. 1999;48(8):615-9.

101. Sollner W, Maislinger S, DeVries A, et al. Use of complementary and alternative medicine by cancer patients is not associated with perceived distress or poor compliance with standard treatment but with active coping behavior: A survey. Cancer. 2000;89(4):873-80.

102. Palinkas LA, Kabongo ML. San Diego Unified Practice in Family Medicine. The use of complementary and alternative medicine by primary care patients. A SURF*NET study. J Fam Pract. 2000;49(12):1221-130.

103. Kanning M. Complementary and alternative therapies for rheumatic diseases I. Part 1. Issues. Why I would want to use complementary and alternative therapy: A patient's perspective. Rheum Dis Clin North America. 1999;25(4):823-30.

104. NIH. Complementary, Alternative, or Integrative Health: What's In a Name? Bethesda, MD2015 [cited 2015 4/13]. Available from: https://nccih.nih.gov/health/integrative-health.

105. Braverman DL, Ericken JJ, Shah RV, Franklin DJ. Interventions in chronic pain management 3. New frontiers in pain management: complementary techniques. Arch Phys Med Rehabil. 2003;84(3 Suppl 1):S45-9.

106. Eisenberg DM, Davis RB, Ettner SL, Appel S, Wilkey S, Van Rompay M, et al. Trends in alternative medicine use in the United States, 1990-1997: results of a follow-up of a national survey. JAMA. 1998;280:1569-75.

107. Institute of Medicine (U.S.). Committee on the Use of Complementary and Alternative Medicine by the American Public. Complementary and alternative medicine in the United States.

Washington, DC: National Academies Press; 2005. xx, 337 p. p.

108. Kirkland J, Matthews, Holly F., Sulivan III, C.W., Baldwin, Karen. Herbal and Magical Medicine: Traditional Healing Today: Duke University Press; 1992.

109. Nahin RL, Barnes PM, Stussman BJ, Bloom B. Costs of complementary and alternative medicine (CAM) and frequency of visits to CAM practitioners: United States, 2007. National health statistics reports. 2009(18):1-14. PubMed PMID: 19771719. 
110. Barnes PM, Powell-Griner E, McFann K, Nahin RL. Complementary and alternative medicine use among adults: United States 2002. Hyattsville, MD: National Center for Health Statistics, 2004 Contract No.: Report.

111. Arcury Q, Bell \& Vitolins. Complementary and alternativemedicine use among rural adults. Complementary Health Practice Review. 2002;7(3):167-86.

112. Bausell RB, Lee WL, Berman BM. Demographic and health-related correlates to visits to complementary and alternative medical providers. Medical care. 2001;39(2):190-6. PubMed PMID: 11176556.

113. Graham RE, Ahn AC, Davis RB, O'Connor BB, Eisenberg DM, Phillips RS. Use of complementary and alternative medical therapies among racial and ethnic minority adults: results from the 2002 National Health Interview Survey. J Natl Med Assoc. 2005;97(4):535-45. PubMed PMID: 15868773; PubMed Central PMCID: PMC2568705.

114. Montalto CP, Bhargava, V., \& Hong, G. S. Use of complementary and alternative medicineby older adults: An exploratory study. Complementary Health Practice Review. 2006;11(1):27-46.

115. Tindle HA, Davis RB, Phillips RS, Eisenberg DM. Trends in use of complementary and alternative medicine by US adults: 1997-2002. Altern Ther Health Med. 2005;11(1):42-9. PubMed PMID: 15712765.

116. Bhargava V, Hong GS, Montalto CP. Use of practitioner-based and self-care complementary and alternative medicine in the united states: A demand for health perspective. Family and Consumer Sciences Research Journal. 2012;41(1):18-35. doi: 10.1111/j.1552-3934.2012.02126.x.

117. Schuster TL, Dobson M, Jauregui M, Blanks RH. Wellness lifestyles I: A theoretical framework linking wellness, health lifestyles, and complementary and alternative medicine. J Altern Complement Med. 2004;10(2):349-56. doi: 10.1089/107555304323062347. PubMed PMID: 15165416.

118. Davis MA, West AN, Weeks WB, Sirovich BE. Health Behaviors and Utilization among Users of Complementary and Alternative Medicine for Treatment versus Health Promotion. Health services research. 2011;46(5):1402-16. doi: 10.1111/j.1475-6773.2011.01270.x.

119. Green J. The herbal medicine-makers' handbook : a home manual. Freedom, Calif.: The Crossing Press; 2000. 384 p. p.

120. Cech R. [Growing medicinal plants]. Williams, OR: Horizon Herbs; 1995. v. <1-7, 9, 11$\rangle$ p.

121. Amjad H. Folk Medicine of Appalachia: A Vanishing Tradition: lulu.com (self-published); 2006. $340 \mathrm{p}$.

122. Rehder JB. Appalachian folkways. Baltimore: Johns Hopkins University Press; 2004. x, 353 p. p.

123. Audette JF, Bailey A. Integrative pain medicine : the science and practice of complementary and alternative medicine in pain management. Totowa, N.J.: Humana; 2008. xiv, 579 p. p.

124. Cavender A. Folk Medicine in Southern Appalachia: The University of North Carolina Press; 2003. 266 p.

125. Wu CH, Wang CC, Tsai MT, Huang WT, Kennedy J. Trend and pattern of herb and supplement use in the United States: results from the 2002, 2007, and 2012 national health interview surveys. Evidence-based complementary and alternative medicine : eCAM. 2014;2014:872320. doi: 10.1155/2014/872320. PubMed PMID: 25574184; PubMed Central PMCID: PMC4276694.

126. Kessler RC, Davis RB, Foster DF, Van Rompay MI, Walters EE, Wilkey SA, et al. Long-term trends in the use of complementary and alternative medical therapies in the United States. Ann Intern Med. 2001;135(4):262-8. Epub 2001/08/21. PubMed PMID: 11511141.

127. Kennedy J. Herb and supplement use in the US adult population. Clin Ther. 2005;27(11):184758. doi: 10.1016/j.clinthera.2005.11.004. PubMed PMID: 16368456.

128. Schaffer DM, Gordon NP, Jensen CD, Avins AL. Nonvitamin, nonmineral supplement use over a 12-month period by adult members of a large health maintenance organization. J Am Diet Assoc. 2003;103(11):1500-5. Epub 2003/10/25. doi: 10.1016/s0002. PubMed PMID: 14576716. 
129. Kelly JP, Kaufman DW, Kelley K, Rosenberg L, Mitchell AA. Use of herbal/natural supplements according to racial/ethnic group. J Altern Complement Med. 2006;12(6):555-61. Epub 2006/08/04. doi: 10.1089/acm.2006.12.555. PubMed PMID: 16884347.

130. Tanaka MJ, Gryzlak BM, Zimmerman MB, Nisly NL, Wallace RB. Patterns of natural herb use by Asian and Pacific Islanders. Ethn Health. 2008;13(2):93-108. Epub 2008/04/22. doi: 10.1080/13557850701830349. PubMed PMID: 18425709.

131. Dailey RK, Neale AV, Northrup J, West P, Schwartz KL. Herbal product use and menopause symptom relief in primary care patients: a MetroNet study. Journal of women's health. 2003;12(7):633-41. Epub 2003/10/30. doi: 10.1089/154099903322404285. PubMed PMID: 14583104.

132. Mackenzie ER, Taylor L, Bloom BS, Hufford DJ, Johnson JC. Ethnic minority use of complementary and alternative medicine (CAM): a national probability survey of CAM utilizers. Altern Ther Health Med. 2003;9(4):50-6. Epub 2003/07/19. PubMed PMID: 12868252.

133. Hung OL, Shih RD, Chiang WK, Nelson LS, Hoffman RS, Goldfrank LR. Herbal preparation use among urban emergency department patients. Acad Emerg Med. 1997;4(3):209-13. Epub 1997/03/01. PubMed PMID: 9063549.

134. Klepser TB, Doucette WR, Horton MR, Buys LM, Ernst ME, Ford JK, et al. Assessment of patients' perceptions and beliefs regarding herbal therapies. Pharmacotherapy. 2000;20(1):83-7. Epub 2000/01/21. PubMed PMID: 10641978.

135. Harnack LJ, Rydell SA, Stang J. Prevalence of use of herbal products by adults in the Minneapolis/St Paul, Minn, metropolitan area. Mayo Clin Proc. 2001;76(7):688-94. Epub 2001/07/11. doi: 10.4065/76.7.688. PubMed PMID: 11444400.

136. Bair YA, Gold EB, Greendale GA, Sternfeld B, Adler SR, Azari R, et al. Ethnic differences in use of complementary and alternative medicine at midlife: longitudinal results from SWAN participants. Am J Public Health. 2002;92(11):1832-40. Epub 2002/10/31. PubMed PMID: 12406817; PubMed Central PMCID: PMCPMC1447337.

137. Sawni A, Thomas R. Pediatricians' attitudes, experience and referral patterns regarding Complementary/Alternative Medicine: a national survey. BMC Complement Altern Med. 2007;7:18. doi: 10.1186/1472-6882-7-18. PubMed PMID: 17547752; PubMed Central PMCID: PMC1894987.

138. Tom Xu K, Farrell TW. The complementarity and substitution between unconventional and mainstream medicine among racial and ethnic groups in the United States. Health Serv Res. 2007;42(2):811-26. Epub 2007/03/17. doi: 10.1111/j.1475-6773.2006.00628.x. PubMed PMID: 17362219; PubMed Central PMCID: PMCPMC1955362.

139. Chao MT, Wade C, Kronenberg F. Disclosure of complementary and alternative medicine to conventional medical providers: Variation by race/ethnicity and type of CAM. Journal of the National Medical Association. 2008;100(11):1341-9.

140. Upchurch DM, Chyu L, Greendale GA, Utts J, Bair YA, Zhang G, et al. Complementary and alternative medicine use among American women: Findings from the National Health Interview Survey, 2002. Journal of women's health. 2007;16(1):102-13. doi: 10.1089/jwh.2006.M074.

141. Blalock et al. Factors Associated with Potential Medication-Herb/natural Product Interactions in a Rural Community. Alternative Therapies in Health \& Medicine. 2009;15(5):26-34.

142. Nguyen LT, Davis RB, Kaptchuk TJ, Phillips RS. Use of complementary and alternative medicine and self-rated health status: Results from a national survey. Journal of General Internal Medicine. 2011;26(4):399-404. doi: 10.1007/s11606-010-1542-3.

143. Nguyen HT, Grzywacz JG, Lang W, Walkup M, Arcury TA. Effects of Complementary Therapy on Health in a National U.S. Sample of Older Adults. Journal of Alternative \& Complementary Medicine. 2010;16(7):701-6. doi: 10.1089/acm.2009.0442. 
144. Neiberg RH, Aickin M, Grzywacz JG, Lang W, Quandt SA, Bell RA, et al. Occurrence and cooccurrence of types of complementary and alternative medicine use by age, gender, ethnicity, and education among adults in the United States: The 2002 national health interview survey (NHIS).

Journal of Alternative and Complementary Medicine. 2011;17(4):363-70. doi: 10.1089/acm.2009.0157.

145. Nahin RL, Dahlhamer JM, Taylor BL, Barnes PM, Stussman BJ, Simile CM, et al. Health behaviors and risk factors in those who use complementary and alternative medicine. BMC Public Health. 2007;7:217. doi: 10.1186/1471-2458-7-217. PubMed PMID: 17723149; PubMed Central PMCID: PMC2031902.

146. Nahin RL, Dahlhamer JM, Stussman BJ. Health need and the use of alternative medicine among adults who do not use conventional medicine. BMC Health Serv Res. 2010;10:220. doi: 10.1186/1472-6963-10-220. PubMed PMID: 20670418; PubMed Central PMCID: PMC2919531.

147. Misra R, Balagopal P, Klatt M, Geraghty M. Complementary and alternative medicine use among Asian Indians in the United States: A national study. Journal of Alternative and Complementary Medicine. 2010;16(8):843-52. doi: 10.1089/acm.2009.0517.

148. Mehta DH, Phillips RS, Davis RB, McCarthy EP. Use of complementary and alternative therapies by Asian Americans. Results from the National Health Interview Survey. Journal of General Internal Medicine. 2007;22(6):762-7. doi: 10.1007/s11606-007-0166-8.

149. Kennedy J, Wang CC, Wu CH. Patient Disclosure about Herb and Supplement Use among Adults in the US. Evidence-based complementary and alternative medicine : eCAM. 2008;5(4):451-6. doi: 10.1093/ecam/nem045. PubMed PMID: 18955213; PubMed Central PMCID: PMC2586312.

150. Kannan VD, Gaydos LM, Atherly AJ, Druss BG. Medical utilization among wellness . Med Care Res Rev. 2010;67(6):722-36. Epub 2010/06/04. doi: 10.1177/1077558710370706. PubMed PMID: 20519427.

151. Jones L, Sciamanna C, Lehman E. Are those who use specific complementary and alternative medicine therapies less likely to be immunized? Preventive medicine. 2010;50(3):148-54. doi: 10.1016/j.ypmed.2009.12.001.

152. Barner JC, Bohman TM, Brown CM, Richards KM. Use of complementary and alternative medicine for treatment among African-Americans: A multivariate analysis. Research in Social and Administrative Pharmacy. 2010;6(3):196-208. doi: 10.1016/j.sapharm.2009.08.001.

153. Hildreth KD EC. Alternative worldviews and the utilization of conventional and complementary medicine. Sociological Inquiry. 2007;77(1):76-103.

154. Birdee GS, Phillips RS, Davis RB, Gardiner P. Factors associated with pediatric use of complementary and alternative medicine. Pediatrics. 2010;125(2):249-56. doi: 10.1542/peds.2009-1406.

155. Grzywacz JG, Lang W, Suerken C, Quandt SA, Bell RA, Arcury TA. Age, race, and ethnicity in the use of complementary and alternative medicine for health self-management: evidence from the 2002 National Health Interview Survey. Journal of aging and health. 2005;17(5):547-72. Epub 2005/09/24. doi: 10.1177/0898264305279821. PubMed PMID: 16177450.

156. Grzywacz JG, Suerken CK, Neiberg RH, Lang W, Bell RA, Quandt SA, et al. Age, ethnicity, and use of complementary and alternative medicine in health self-management. Journal of health and social behavior. 2007;48(1):84-98. PubMed PMID: 17476925.

157. Gardiner et al. Factors Associated with Herbal Therapy use by Adults in the United States. Alternative Therapies in Health \& Medicine. 2007;13(2):22-9.

158. Elmer GW, Lafferty WE, Tyree PT, Lind BK. Potential interactions between complementary/alternative products and conventional medicines in a Medicare population. Ann 
Pharmacother. 2007;41(10):1617-24. Epub 2007/09/06. doi: 10.1345/aph.1K221. PubMed PMID: 17785609; PubMed Central PMCID: PMCPMC2864004.

159. Ellison CG, Bradshaw M, Roberts CA. Spiritual and religious identities predict the use of complementary and alternative medicine among US adults. Preventive medicine. 2012;54(1):912. doi: 10.1016/j.ypmed.2011.08.029.

160. Upchurch DM, Dye CE, Chyu L, Gold EB, Greendale GA. Demographic, behavioral, and health correlates of complementary and alternative medicine and prayer use among midlife women: 2002. Journal of women's health. 2010;19(1):23-30. doi: 10.1089/jwh.2008.1096. PubMed PMID: 20088655; PubMed Central PMCID: PMC2828262.

161. Barnes PM, Bloom B, Nahin RL. Complementary and alternative medicine use among adults and children: United States, 2007. National health statistics reports. 2008(12):1-23. Epub 2009/04/14. PubMed PMID: 19361005.

162. Bardia A, Nisly NL, Zimmerman MB, Gryzlak BM, Wallace RB. Use of herbs among adults based on evidence-based indications: findings from the National Health Interview Survey. Mayo Clin Proc. 2007;82(5):561-6. Epub 2007/05/12. doi: 10.4065/82.5.561. PubMed PMID: 17493422; PubMed Central PMCID: PMCPMC1964882.

163. Arcury TA, Grzywacz JG, Bell RA, Neiberg RJ, Lang W, Quandt SA. Herbal remedy use as health self-management among older adults. Journals of Gerontology: Series B: Psychological Sciences and Social Sciences. 2007;62B(2):S142-S9.

164. Brown CM, Barner JC, Richards KM, Bohman TM. Patterns of complementary and alternative medicine use in African Americans. Journal of Alternative and Complementary Medicine. 2007;13(7):751-8. doi: 10.1089/acm.2006.6392.

165. Mehta DH, Gardiner PM, Phillips RS, McCarthy EP. Herbal and dietary supplement disclosure to health care providers by individuals with chronic conditions. J Altern Complement Med. 2008;14(10):1263-9. Epub 2008/11/27. doi: 10.1089/acm.2008.0290. PubMed PMID: 19032071; PubMed Central PMCID: PMCPMC2787410.

166. Clarke TC, Black LI, Stussman BJ, Barnes PM, Nahin RL. Trends in the use of complementary health approaches among adults: United States, 2002-2012. National health statistics reports. 2015(79):1-16. PubMed PMID: 25671660.

167. Commission AR. The Appalachian Region 2015 [cited 2015]. Available from: http://www.arc.gov/appalachian_region/TheAppalachianRegion.asp.

168. Crellin JK, Philpott J, Crellin JK. A reference guide to medicinal plants : herbal medicine past and present. Durham, NC: Duke University Press; 1997. 551 p. p.

169. Crellin JK, Bass ALT, Philpott J. Trying to give ease : Tommie Bass and the story of herbal medicine. Durham: Duke University Press; 1997. xii. 335 p. p.

170. Allen JL. Through Cumberland Gap on Horseback. Harper's Magazine. June 1886:50-66.

171. Koehler JH. Ginseng and goldenseal growers' handbook. Wausau, Wis.,: P. F. Stolze; 1912. vii, 116 p. p.

172. Rago LO. Blackberry Cove herbal : healing with common herbs in the Appalachian wise-woman tradition. Sterling, Va.: Capital Books; 2000. ix, 166 p. p.

173. Growing \& Marketing Ginseng, Goldenseal \& Other Woodland Medicinals. North Carolina Libraries (Online). 2006;64(1/2):35-. PubMed PMID: 21528280.

174. Persons WS, Davis JM. Growing \& marketing ginseng, goldenseal \& other woodland medicinals. Fairview, N.C.: Bright Mountain Books; 2005. xii, 466 p. p.

175. Atkins ABS. "She Didn't Go Sangin' Alone!". Goldenseal. 1999:27-9.

176. Amjad H. Life \& Thymes of an Appalachian Herbalist: lulu.com (self-published); 2006.

177. Flynn MHJ. Tending the Commons: Folklife and Landscape in Southern West Virginia1993. Available from: http://www.loc.gov/collections/folklife-and-landscape-in-southernwesthttp://www.loc.gov/collections/folklife-and-landscape-in-southern-west-virginia/articles- 
and-essays/american-ginseng-and-the-idea-of-the-commons/virginia/articles-and-

essays/american-ginseng-and-the-idea-of-the-commons/.

178. Goodwin JG. Going 'Senging. Goldenseal. 1985:16-21.

179. Bolyard JL. Medicinal plants and home remedies of Appalachia. Springfield, Ill.: Thomas; 1981. xvii, 187 p. p.

180. Collins KC, Hunter L. Foxfire 11 : the old homeplace, wild plant uses, preserving and cooking food, hunting stories, fishing, and more affairs of plain living. 1st ed. New York: Anchor Books; 1999. xi, 313 p. p.

181. Moerman DE. Native American Medicinal Plants: An Ethnobotanical Dictionary. First Abridged Edition ed. Portland, OR: Timber Press, Inc.; 2009. 799 p.

182. Wilson CR, Ferris WR. Encyclopedia of Southern culture. Chapel Hill: University of North Carolina Press; 1989. xxi, 1634 p. p.

183. Cook C, Baisden D. Ancillary Use of Folk Medicine by Patients in Primary Care Clinics in Southwestern West-Virginia. Southern Med J. 1986;79(9):1098-101. PubMed PMID: WOS:A1986E085900013.

184. Glover DD, Amonkar M, Rybeck BF, Tracy TS. Prescription, over-the-counter, and herbal medicine use in a rural, obstetric population. American journal of obstetrics and gynecology. 2003;188(4):1039-45. doi: http://dx.doi.org/10.1067/mob.2003.223.

185. Cool R. Folk Medicine in the Kentucky Hills. Appalachian Heritage. 1976:51-6.

186. Ward B. Folk Medicine: West Virginia Humanities Council; 2010 [cited 2015 April 21]. Available from: http://www.wvencyclopedia.org/articles/2221.

187. Deskins C. Healing from the Hills. Goldenseal. 1990:60-4.

188. Tanner L. Clay County Folklore and Folk Medicine: Empty Rockers \& Sassafras Tea. Goldenseal. 1995:67-9.

189. Ackers-Johnson M, Talasila A, Sage AP, Long X, Bot I, Morrell NW, et al. Myocardin regulates vascular smooth muscle cell inflammatory activation and disease. Arteriosclerosis, thrombosis, and vascular biology. 2015;35(4):817-28. doi: 10.1161/ATVBAHA.114.305218. PubMed PMID: 25614278.

190. Seaton CT. Hippie homesteaders : arts, crafts, music, and living on the land in West Virginia. First Edition. ed. Morgantown, West Virginia: West Virginia University Press; 2014. xiii, 281 pages $\mathrm{p}$.

191. Bennett TGaA. Catfish: Portrait of an Herb Doctor. Goldenseal. 1977:46-51.

192. Ernst E. Usage of complementary therapies in rheumatology: a systematic review. Clinical rheumatology. 1998;17(4):301-5. PubMed PMID: 9776112.

193. Pullar T, Capell HA, Millar A, Brooks RG. Alternative medicine: cost and subjective benefit in rheumatoid arthritis. British medical journal. 1982;285(6355):1629-31. PubMed PMID: 6814682; PubMed Central PMCID: PMC1500773.

194. Ernst E, White A. The BBC survey of complementary medicine use in the UK. Complementary therapies in medicine. 2000;8(1):32-6. doi: http://dx.doi.org/10.1016/S0965-2299(00)90833-1.

195. Higham C, Ashcroft C, Jayson MI. Non-prescribed treatments in rheumatic diseases. The Practitioner. 1983;227(1381):1201-5. PubMed PMID: 6604270.

196. Dimmock S, Troughton PR, Bird HA. Factors predisposing to the resort of complementary therapies in patients with fibromyalgia. Clinical rheumatology. 1996;15(5):478-82. PubMed PMID: 8894361.

197. Shaver JL, Wilbur J, Lee H, Robinson FP, Wang E. Self-reported medication and herb/supplement use by women with and without fibromyalgia. Journal of women's health. 2009;18(5):709-16. doi: 10.1089/jwh.2008.1194. PubMed PMID: 19445618.

198. A Blueprint for Transforming Prevention, Care, Education, and Research. Washington, DC: Institute of Medicine, 2012 June 2011. 
199. Mason L, Moore RA, Derry S, Edwards JE, McQuay HJ. Systematic review of topical capsaicin for the treatment of chronic pain. BMJ. 2004;328(7446):991. doi:

10.1136/bmj.38042.506748.EE.

PubMed PMID: 15033881; PubMed Central PMCID: PMC404499.

200. Miyasaka Lincoln S, N AÁ, Soares B. : Passiflora for anxiety disorder. : Cochrane Database of Systematic Reviews.

201. Miyasaka Lincoln S, N AÁ, Soares B. : Valerian for anxiety disorders. : Cochrane Database of Systematic Reviews.

202. H PM, Ernst E. : Kava extract versus placebo for treating anxiety. : Cochrane Database of Systematic Reviews.

203. Cui X, Trinh K, Wang Y-J. : Chinese herbal medicine for chronic neck pain due to cervical degenerative disc disease. : Cochrane Database of Systematic Reviews.

204. Pittler MH, Ernst E. Feverfew for preventing migraine. The Cochrane database of systematic reviews. 2004(1):CD002286. doi: 10.1002/14651858.CD002286.pub2. PubMed PMID: 14973986.

205. Wider B, Pittler MH, Ernst E. Feverfew for preventing migraine. The Cochrane database of systematic reviews. 2015;4:CD002286. doi: 10.1002/14651858.CD002286.pub3. PubMed PMID: 25892430.

206. Wider B, H PM, Ernst E. : Feverfew for preventing migraine. : Cochrane Database of Systematic Reviews.

207. Linde K, M BM, Kriston L. : St John's wort for major depression. : Cochrane Database of Systematic Reviews.

208. Oltean H, Robbins C, W vTM, M BB, Bombardier C, J GJ. : Herbal medicine for low-back pain: Cochrane Database of Systematic Reviews.

209. Liu Jian P, Yang M, Liu Y, Wei Mao L, Grimsgaard S. : Herbal medicines for treatment of irritable bowel syndrome. : Cochrane Database of Systematic Reviews.

210. Cameron M, J GJ, Chrubasik S. : Herbal therapy for treating rheumatoid arthritis. : Cochrane Database of Systematic Reviews.

211. Cabrera C. Fibromyalgia : a journey toward healing. Chicago: Contemporary Books; 2002. xiii, 318 p. p.

212. Brunvand JH. American folklore : an encyclopedia. New York: Garland Pub.; 1996. xviii, 794 p. p.

213. Duke University. Library. Frank C. Brown Collection of North Carolina Folklore., White NI, Brown FC, North Carolina Folklore Society. The Frank C. Brown Collection of North Carolina Folklore; the folklore of North Carolina, collected by Dr. Frank C. Brown during the years 1912 to 1943, in collaboration with the North Carolina Folklore Society. Durham, N.C.,: Duke University Press; 1952.

214. Puckett NN, Hand WD, Casetta A, Thiederman SB. Popular beliefs and superstitions : a compendium of American folklore : from the Ohio Collection of Newbell Niles Puckett. Boston, Mass.: G.K. Hall; 1981.

215. Browne RB. Popular beliefs and practices from Alabama. Berkeley,: University of California Press; 1958. 271 p. p.

216. Shelton F. Pioneer comforts and kitchen remedies;: Oldtimey highland secrets from the Blue Ridge and Great Smoky Mountains: Hutcraft; 1965. 24 p.

217. Crellin JK, Philpott J, Bass ALT. Herbal medicine past and present. Durham: Duke University Press; 1990.

218. Krochmal A, Walters RS, Doughty RM. A guide to medicinal plants of Appalachia. Washington,: U.S. Forest Service; for sale by the Supt. of Docs.; 1971. 291 p. p. 
219. Stephenson SL. A natural history of the central Appalachians. 1st ed. Morgantown: West Virginia University Press; 2013. xi, 259 p. p.

220. Chrubasik S, Kunzel O, Black A, Conradt C, Kerschbaumer F. Potential economic impact of using a proprietary willow bark extract in outpatient treatment of low back pain: an open nonrandomized study. Phytomedicine. 2001;8(4):241-51. doi: 10.1078/0944-7113-00048. PubMed PMID: 11515713.

221. Chrubasik S, Eisenberg E, Balan E, Weinberger T, Luzzati R, Conradt C. Treatment of low back pain exacerbations with willow bark extract: a randomized double-blind study. The American journal of medicine. 2000;109(1):9-14. PubMed PMID: 10936472.

222. Schmid B, Ludtke R, Selbmann HK, Kotter I, Tschirdewahn B, Schaffner W, et al. Efficacy and tolerability of a standardized willow bark extract in patients with osteoarthritis: randomized placebo-controlled, double blind clinical trial. Phytother Res. 2001;15(4):344-50. PubMed PMID: 11406860.

223. World Health Organization. WHO monographs on selected medicinal plants. Geneva: World Health Organization; 1999-2009. v. <1-4> p.

224. Hyson MI. Anticephalgic photoprotective premedicated mask. A report of a successful doubleblind placebo-controlled study of a new treatment for headaches with associated frontalis pain and photophobia. Headache. 1998;38(6):475-7. PubMed PMID: 9664755.

225. Organization WH. WHO Monographs on Slected Medicinal Plants. 2003.

226. Carle R GK. Chamomile: a pharmacological and clinical profile. Drugs of Today. 1992;28:55965.

227. Gould L RC, Gomprecht RF. Cardiac effect of chamomile tea. Journal of clinical pharmacology. 1973;13:475-9.

228. Chadwick LR, Pauli GF, Farnsworth NR. The pharmacognosy of Humulus lupulus L. (hops) with an emphasis on estrogenic properties. Phytomedicine : international journal of phytotherapy and phytopharmacology. 2006;13(1-2):119-31. doi: 10.1016/j.phymed.2004.07.006. PubMed PMID: PMC1852439.

229. al. LKe. Effects of Humulus lupulus extract on the central nervous system in mice. Planta medica. 1993;59(Suppl.):A691.

230. al. LKe. Neuropharmacological activity of Humulus lupulus extracts. Korean Journal of Pharmacognosy. 1993;24:231-4.

231. Belch JJ, Ansell D, Madhok R, O'Dowd A, Sturrock RD. Effects of altering dietary essential fatty acids on requirements for non-steroidal anti-inflammatory drugs in patients with rheumatoid arthritis: a double blind placebo controlled study. Annals of the rheumatic diseases. 1988;47(2):96-104.

232. Ghazanfar SA. Handbook of Arabian medicinal plants. Boca Raton: CRC Press; 1994. 265 p. p.

233. Srivastava KC, Mustafa T. Ginger (Zingiber officinale) in rheumatism and musculoskeletal disorders. Medical hypotheses.39(4):342-8. doi: 10.1016/0306-9877(92)90059-L.

234. Gobel H, Schmidt G, Soyka D. Effect of peppermint and eucalyptus oil preparations on neurophysiological and experimental algesimetric headache parameters. Cephalalgia : an international journal of headache. 1994;14(3):228-34; discussion 182. PubMed PMID: 7954745.

235. Gobel H, Schmidt G, Dworschak M, Stolze H, Heuss D. Essential plant oils and headache mechanisms. Phytomedicine. 1995;2(2):93-102. doi: 10.1016/S0944-7113(11)80053-X. PubMed PMID: 23196150.

236. Zamora R, Vodovotz Y, Billiar TR. Inducible nitric oxide synthase and inflammatory diseases. Molecular medicine. 2000;6(5):347-73. PubMed PMID: PMC1949959.

237. Pepys MB, Hirschfield GM. C-reactive protein: a critical update. The Journal of clinical investigation. 2003;111(12):1805-12. doi: 10.1172/JCI18921. PubMed PMID: 12813013; PubMed Central PMCID: PMC161431. 
238. Szalai AJ. The biological functions of C-reactive protein. Vascular pharmacology. 2002;39(3):105-7. PubMed PMID: 12616974.

239. Szalai AJ, McCrory MA, Cooper GS, Wu J, Kimberly RP. Association between baseline levels of C-reactive protein (CRP) and a dinucleotide repeat polymorphism in the intron of the CRP gene. Genes and immunity. 2002;3(1):14-9. doi: 10.1038/sj.gene.6363820. PubMed PMID: 11857055.

240. Bucova M, Bernadic M, Buckingham T. C-reactive protein, cytokines and inflammation in cardiovascular diseases. Bratislavske lekarske listy. 2008;109(8):333-40. PubMed PMID: 18837239.

241. Mascolo N, Autore G, Capasso F, Menghini A, Fasulo MP. - Biological screening of Italian medicinal plants for anti-inflammatory activity. 1987;- 1(- 1):- 31 .

242. al. TBe. Taraxacum offi cinale W.: Pharmacological effect of an ethanol extract. Pharmacology Research. 1993;27(Suppl.1):23-4.

243. Yasukawa K, Yamaguchi A, Arita J, Sakurai S, Ikeda A, Takido M. - Inhibitory effect of edible plant extracts on 12-O-tetradecanoylphorbol-13-acetate-induced ear oedema in mice. 1993;- 7(2):- 189.

244. Delaveau P, Lallouette P, Tessier AM. [Stimulation of the phagocytic activity of R.E.S. by plant extracts (author's transl)]. Planta medica. 1980;40(1):49-54. doi: 10.1055/s-2008-1074941. PubMed PMID: 6999511.

245. Yesilada E, Ustun O, Sezik E, Takaishi Y, Ono Y, Honda G. Inhibitory effects of Turkish folk remedies on inflammatory cytokines: interleukin-1alpha, interleukin-1beta and tumor necrosis factor alpha. J Ethnopharmacol. 1997;58(1):59-73. PubMed PMID: 9324006.

246. al. WHe. In vitro inhibition of prostaglandin biosynthesis by essential oils and phenolic compounds. Plant Medica. 1986;3:184-7.

247. Yasukawa K, Takeuchi M, Takido M. Humulon, a bitter in the hop, inhibits tumor promotion by 12-O-tetradecanoylphorbol-13-acetate in two-stage carcinogenesis in mouse skin. Oncology. 1995;52(2):156-8. PubMed PMID: 7854777.

248. H. H. Economic and medicinal plant research. Wagner H HH, Farnsworth NR, editor. London: Academic Press; 1985.

249. Duwiejua M, Zeitlin IJ, Waterman PG, Gray AI. Anti-inflammatory activity of Polygonum bistorta, Guaiacum officinale and Hamamelis virginiana in rats. The Journal of pharmacy and pharmacology. 1994;46(4):286-90. PubMed PMID: 8051612.

250. Cuellar MJ, Giner RM, Recio MC, Manez S, Rios JL. Topical anti-inflammatory activity of some Asian medicinal plants used in dermatological disorders. Fitoterapia. 2001;72(3):221-9. PubMed PMID: 11295297.

251. Butenko IG, Gladtchenko SV, Galushko SV. Anti-inflammatory properties and inhibition of leukotriene $\mathrm{C} 4$ biosynthesis in vitro by flavonoid baicalein from Scutellaria baicalensis georgy roots. Agents and actions. 1993;39 Spec No:C49-51. PubMed PMID: 8273583.

252. Kimura Y, Yokoi K, Matsushita N, Okuda H. Effects of flavonoids isolated from scutellariae radix on the production of tissue-type plasminogen activator and plasminogen activator inhibitor 1 induced by thrombin and thrombin receptor agonist peptide in cultured human umbilical vein endothelial cells. The Journal of pharmacy and pharmacology. 1997;49(8):816-22. Epub 1997/08/01. PubMed PMID: 9379363.

253. al. URe. American herbal pharmacopeia. Santa Cruz, CA: American Herbal Pharmacopeia; 1999.

254. Wagner I, Greim C, Laufer S, Heide L, Gleiter CH. Influence of willow bark extract on cyclooxygenase activity and on tumor necrosis factor alpha or interleukin 1 beta release in vitro and ex vivo. Clin Pharmacol Ther. 2003;73(3):272-4. Epub 2003/03/07. doi:

10.1067/mcp.2003.32. PubMed PMID: 12621392.

255. Chung CP, Park JB, Bae KH. Pharmacological effects of methanolic extract from the root of Scutellaria baicalensis and its flavonoids on human gingival fibroblast. Planta medica. 
1995;61(2):150-3. Epub 1995/04/01. doi: 10.1055/s-2006-958036. PubMed PMID: 7753922.

256. Cheng GF, Liu DP, Yang DX, He KQ, Bai JY, Zhu XY. Antiinflammatory effects of Tremulacin, a Salicin-related substance isolated from Populus tomentosa Carr. leaves. Phytomedicine. 1994;1(3):209-11. doi: 10.1016/S0944-7113(11)80067-X. PubMed PMID: 23195941.

257. Srivastava KC. Aqueous extracts of onion, garlic and ginger inhibit platelet aggregation and alter arachidonic acid metabolism. Biomed Biochim Acta. 1984;43(8-9):S335-46. PubMed PMID: 6440548.

258. Mustafa T SK, Jensen KB. Drug development report 9. Pharmacology of ginger, Zingiber officinale. Journal of Drug Development. 1993;6:25-39.

259. Suekawa M, Yuasa K, Isono M, Sone H, Ikeya Y, Sakakibara I, et al. [Pharmacological studies on ginger. IV. Effect of (6)-shogaol on the arachidonic cascade]. Nihon yakurigaku zasshi Folia pharmacologica Japonica. 1986;88(4):263-9. Epub 1986/10/01. PubMed PMID: 3098654.

260. Sharma JN, Srivastava KC, Gan EK. Suppressive effects of eugenol and ginger oil on arthritic rats. Pharmacology. 1994;49(5):314-8. PubMed PMID: 7862743.

261. Shunro K, Morimitsu Y, Toshihiko O. Chemistry of Ginger Components and Inhibitory Factors of the Arachidonic Acid Cascade. Food Phytochemicals for Cancer Prevention II. ACS Symposium Series. 547: American Chemical Society; 1994. p. 244-50.

262. Wang M, Guilbert LJ, Ling L, Li J, Wu Y, Xu S, et al. Immunomodulating activity of CVTE002, a proprietary extract from North American ginseng (Panax quinquefolium). The Journal of pharmacy and pharmacology. 2001;53(11):1515-23. PubMed PMID: 11732754.

263. Assinewe VA, Amason JT, Aubry A, Mullin J, Lemaire I. Extractable polysaccharides of Panax quinquefolius L. (North American ginseng) root stimulate TNFalpha production by alveolar macrophages. Phytomedicine. 2002;9(5):398-404. PubMed PMID: 12222658.

264. GB L. A pharmacometric evaluation of nine Bio-Strath herbal remedies. Medita. 1978;8:3-19.

265. al CBe. Pharmacological properties of saponin fractions obtained from domestic crude drugs: Saponaria officinalis, Primula officinalis and Aesculus hippocastanum. Herba Polonica. 1976;22:154-62.

266. J B. Pharmacognosy, phytochemistry, medicinal plants. Paris: Lavoisier; 1995.

267. NG B. Max Wichtl's herbal drugs \& phytopharmaceuticals. Boca Raton, FL: CRC Press; 1994.

268. Bauer R WH. Echinacea species as potential immunostimulatory drugs. Economic and medicinal plants research. London: Academic Press; 1991. p. 253-321.

269. Arafa NM, Hamuda HM, Melek ST, Darwish SK. The effectiveness of Echinacea extract or composite glucosamine, chondroitin and methyl sulfonyl methane supplements on acute and chronic rheumatoid arthritis rat model. Toxicology and industrial health. 2013;29(2):187-201. doi: 10.1177/0748233711428643. PubMed PMID: 22173958.

270. Wang H, Guo Y, Zhao X, Li H, Fan G, Mao H, et al. An estrogen receptor dependent mechanism of Oroxylin A in the repression of inflammatory response. PLoS One. 2013;8(7):e69555. doi: 10.1371/journal.pone.0069555. PubMed PMID: 23922737; PubMed Central PMCID: PMC3726624.

271. NIH. Thunder God Vine 2012 [cited 2015 May 23]. Available from: https://nccih.nih.gov/health/tgvine.

272. Peana AT, D'Aquila PS, Panin F, Serra G, Pippia P, Moretti MD. Anti-inflammatory activity of linalool and linalyl acetate constituents of essential oils. Phytomedicine. 2002;9(8):721-6. doi: 10.1078/094471102321621322. PubMed PMID: 12587692.

273. Halliwell B ZK, Whiteman M The gastrointestinal tract: a major site of antioxidant action? Free Radical Res. 2000;33:819-30.

274. Kratchanova M, Denev P, Ciz M, Lojek A, Mihailov A. Evaluation of antioxidant activity of medicinal plants containing polyphenol compounds. Comparison of two extraction systems. Acta biochimica Polonica. 2010;57(2):229-34. PubMed PMID: 20532255. 
275. Ciz M CH, Denev P, Kratchanova M, Slavov A, Lojek A Different methods for control and comparison of the antioxidant properties of vegetables. Food Control. 2010;21:518-23.

276. Denev P LA, Ciz M, Kratchanova M Antioxidant activity and polyphenol content of Bulgarian fruits. Bulgarian Journal of Agricultural Sciences. 2013;19:22-7.

277. Denev P, Kratchanova M, Ciz M, Lojek A, Vasicek O, Blazheva D, et al. Antioxidant, antimicrobial and neutrophil-modulating activities of herb extracts. Acta biochimica Polonica. 2014;61(2):359-67. PubMed PMID: 24945135.

278. Suzuki H, Nishizawa T, Tsugawa H, Mogami S, Hibi T. Roles of oxidative stress in stomach disorders. J Clin Biochem Nutr. 2012;50(1):35-9. doi: 10.3164/jcbn.11-115SR. PubMed PMID: 22247598; PubMed Central PMCID: PMC3246180.

279. Popovic Z, Matic R, Bojovic S, Stefanovic M, Vidakovic V. Ethnobotany and herbal medicine in modern complementary and alternative medicine: An overview of publications in the field of I\&C medicine 2001-2013. J Ethnopharmacol. 2016;181:182-92. doi: 10.1016/j.jep.2016.01.034. PubMed PMID: 26807912.

280. Wu CH, Wang CC, Kennedy J. Changes in herb and dietary supplement use in the U.S. adult population: a comparison of the 2002 and 2007 National Health Interview Surveys. Clin Ther. 2011;33(11):1749-58. doi: 10.1016/j.clinthera.2011.09.024. PubMed PMID: 22030445.

281. Bruni F. Dr. Does-It-All: The New York Times; April 16, 2010. Available from: http://www.nytimes.com/2010/04/18/magazine/18Oz-t.html?_r=0.

282. Meeting National Institutes of Health Medicinal Plants Consortium. Current state of the science to help NCCIH set next 5 yr strategic objectives. In: Feinberg T, editor. webinar2015.

\section{Chapter 2}

1. Manchikanti, L., et al., American Society of Interventional Pain Physicians. Comprehensive review of epidemiology, scope, and impact of spinal pain. Pain Physician, 2009. 12: p. E35-70.

2. White, K.P. and M. Harth, Classification, epidemiology, and natural history of fibromyalgia. Current pain and headache reports, 2001. 5(4): p. 320-329.

3. Walitt, B., et al., The Prevalence and Characteristics of Fibromyalgia in the 2012 National Health Interview Survey. PLoS One, 2015. 10(9): p. e0138024.

4. Clauw, D.J., Fibromyalgia and related conditions. Mayo Clin Proc, 2015. 90(5): p. 680-92.

5. Diseases, N.I.o.A.a.M.a.S. Questions and Answers about Fibromyalgia. 2014 [cited 2014 July]; Available from: http://www.niams.nih.gov/Health_Info/Fibromyalgia/default.asp.

6. Bennett, R.M., et al., An internet survey of 2,596 people with fibromyalgia. BMC Musculoskelet Disord, 2007. 8: p. 27.

7. Arnold, L.M., et al., Family study of fibromyalgia. Arthritis Rheum, 2004. 50(3): p. 944-52.

8. Metyas, S.K., J.S. Solyman, and D.G. Arkfeld, Inflammatory Fibromyalgia: Is It Real? Curr Rheumatol Rev, 2015.

9. Bjersing, J.L., M.I. Bokarewa, and K. Mannerkorpi, Profile of circulating microRNAs in fibromyalgia and their relation to symptom severity: an exploratory study. Rheumatol Int, 2015. 35(4): p. 635-42.

10. Fok, E., et al., The Use of an IL-1 Receptor Antagonist Peptide to Control Inflammation in the Treatment of Corneal Limbal Epithelial Stem Cell Deficiency. Biomed Res Int, 2015. 2015: p. 516318.

11. Pepys, M.B. and G.M. Hirschfield, C-reactive protein: a critical update. J Clin Invest, 2003. 111(12): p. 1805-12.

12. Bucova, M., M. Bernadic, and T. Buckingham, C-reactive protein, cytokines and inflammation in cardiovascular diseases. Bratisl Lek Listy, 2008. 109(8): p. 333-40. 
13. Zhang, J.-M. and J. An, Cytokines, Inflammation and Pain. International anesthesiology clinics, 2007. 45(2): p. 27-37.

14. FibroCenter. Fibromyalgia Pain Is Different. 2015 [cited 2015; Available from: http://www.fibrocenter.com/fibromyalgia-pain.aspx.

15. Maes, M. et al., The immune-inflammatory pathophysiology of fibromyalgia: increased serum soluble gp130, the common signal transducer protein of various neurotropic cytokines. Psychoneuroendocrinology, 1999. 24(4): p. 371-383.

16. Wallace D, et al., Cytokines play an aetiopathogenetic role in fibromyalgia: a hypothesis and pilot study. Rheumatology (Oxford), 2001. 40(7): p. 743-749.

17. Gur, A. et al., Regional cerebral flow and cytokines in young females with fibromyalgia. Clinical and experimental rheumatology JID - 8308521, 2002. 20(6): p. 753-760.

18. Gur, A. et al., Cytokines and depression in cases with fibromyalgia. Journal of Rheumatology, 2002. 29(2): p. 358-3561.

19. Hein G, F.S., Are advanced glycaton end-product-modified proteins of pathogentic importance in fibromyalgia. Rheumatology, 2002. 4l(10): p. 1163-1167.

20. Pache M, et al, Increased plasma endothilin-1 levels in fibromyalgia syndrome. Rheumatology, 2003. 42(493-494).

21. Bagis S, t.L.S.G. and et al., Free radicals and antioxidants in primary fibromyalgia: an oxidative stress disorder? Rheumatology international JID - 8206885.

22. Salemi and et al., Detection of interleukin 1 beta (IL-beta), ILO-6, and Tumor necrosis factoralpha in skin of patients with fibromyalgia. Journal of Rheumatology, 2003. 30(1): p. 146150.

23. Kadetoff, D.F., et al., Evidence of central inflammation in fibromyalgia-increased cerebrospinal fluid interleukin-8 levels. 0328.

24. Xiao Y Fau - Haynes, W.L., et al., Elevated serum high-sensitivity C-reactive protein levels in fibromyalgia syndrome patients correlate with body mass index, interleukin-6, interleukin-8, erythrocyte sedimentation rate. 2013.

25. Kaufmann, I.F., et al., Decrease in adhesion molecules on polymorphonuclear leukocytes of patients with fibromyalgia. 0924.

26. Bazzichi, L.F., et al., Cytokine patterns in fibromyalgia and their correlation with clinical manifestations. 0830.

27. Bazzichi, L.F., et al., Cytokine patterns in fibromyalgia and their correlation with clinical manifestations. 2007.

28. Lund Haheim, L.F., et al., C-reactive protein variations for different chronic somatic disorders. 1001.

29. Rus, A., et al., Nitric Oxide, Inflammation, Lipid Profile, and Cortisol in Normal-and Overweight Women With Fibromyalgia. Biol Res Nurs, 2015.

30. Amel Kashipaz, M.R., et al., Normal production of inflammatory cytokines in chronic fatigue and fibromyalgia syndromes determined by intracellular cytokine staining in short-term cultured blood mononuclear cells. Clin Exp Immunol, 2003. 132(2): p. 360-5.

31. Afsar, B.F. and R. Burucu, Urinary albumin, protein excretion and circadian blood pressure in patients with fibromyalgia. 0826.

32. Irwin, M.R., R. Olmstead, and J.E. Carroll, Sleep Disturbance, Sleep Duration, and Inflammation: A Systematic Review and Meta-Analysis of Cohort Studies and Experimental Sleep Deprivation. Biol Psychiatry, 2015.

33. Consoli, G., et al., The impact of mood, anxiety, and sleep disorders on fibromyalgia. Comprehensive psychiatry, 2012. 53(7): p. 962-967.

34. Okifuji, A., et al., Relationship between fibromyalgia and obesity in pain, function, mood, and sleep. J Pain, 2010. 11(12): p. 1329-37. 
35. Dell'Osso, L., et al., The inflammatory hypothesis of mood spectrum broadened to fibromyalgia and chronic fatigue syndrome. Clin Exp Rheumatol, 2015. 33 Suppl 88(1): p. 109-116.

36. Okifuji, A., D.H. Bradshaw, and C. Olson, Evaluating obesity in fibromyalgia: neuroendocrine biomarkers, symptoms, and functions. Clin Rheumatol, 2009. 28(4): p. 475-8.

37. Frisbee, S.J., et al., The C8 health project: design, methods, and participants. Environ Health Perspect, 2009. 117(12): p. 1873-82.

38. Ursini, F., S. Naty, and R. Grembiale, Fibromyalgia and obesity: the hidden link. Rheumatology International, 2011. 31(11): p. 1403-1408.

39. Giacomelli, C., et al., The interaction between autoimmune diseases and fibromyalgia: risk, disease course and management. Expert Rev Clin Immunol, 2013. 9(11): p. 1069-76.

40. Staud, R., Evidence for shared pain mechanisms in osteoarthritis, low back pain, and fibromyalgia. Curr Rheumatol Rep, 2011. 13(6): p. 513-20.

41. Wolfe, F., et al., Chronic conditions and health problems in rheumatic diseases: comparisons with rheumatoid arthritis, noninflammatory rheumatic disorders, systemic lupus erythematosus, and fibromyalgia. J Rheumatol, 2010. 37(2): p. 305-15.

42. Ozgocmen, S., O.B. Cimen, and O. Ardicoglu, Relationship between chest expansion and respiratory muscle strength in patients with primary fibromyalgia. Clin Rheumatol, 2002. 21(1): p. 19-22.

43. Yang, T.Y., et al., Risk for irritable bowel syndrome in fibromyalgia patients: a national database study. Medicine (Baltimore), 2015. 94(10): p. e616.

44. Yanmaz, M.N., M. Mert, and M. Korkmaz, The prevalence of fibromyalgia syndrome in a group of patients with diabetes mellitus. Rheumatol Int, 2012. 32(4): p. 871-4.

45. Michaud, K. and F. Wolfe, The association of rheumatoid arthritis and its treatment with sinus disease. J Rheumatol, 2006. 33(12): p. 2412-5.

46. Tietjen, G.E., et al., Allodynia in migraine: association with comorbid pain conditions. Headache, 2009. 49(9): p. 1333-44.

47. Martinez-Jauand, M., et al., Age-of-onset of menopause is associated with enhanced painful and non-painful sensitivity in fibromyalgia. Clin Rheumatol, 2013. 32(7): p. 975-81.

48. Goesling, J., et al., Associations Between Pain, Current Tobacco Smoking, Depression, and Fibromyalgia Status Among Treatment-Seeking Chronic Pain Patients. Pain Med, 2015. 16(7): p. 1433-42.

49. Prasad, K., C-reactive protein (CRP)-lowering agents. Cardiovasc Drug Rev, 2006. 24(1): p. 3350 .

50. National Heart, L., and Blood Institute. Classification of Overweight and Obesity by BMI, Waist Circumference, and Associated Disease Risks. [cited 2015 February 7]; Available from: https://www.nhlbi.nih.gov/health/educational/lose wt/BMI/bmi dis.htm.

51. Deodhar, A.A., et al., Fluid retention syndrome and fibromyalgia. Br J Rheumatol, 1994. 33(6): p. 576-82.

52. Mork, P.J., O. Vasseljen, and T.I. Nilsen, Association between physical exercise, body mass index, and risk of fibromyalgia: longitudinal data from the Norwegian Nord-Trondelag Health Study. Arthritis Care Res (Hoboken), 2010. 62(5): p. 611-7.

53. Sanchez del Rio-Gonzalez, M., [Chronic migraine: pathophysiology]. Rev Neurol, 2012. 54 Suppl 2: p. S13-9.

54. Lawrence, R.C., et al., Estimates of the prevalence of arthritis and other rheumatic conditions in the United States. Part II. (0004-3591 (Print)).

55. White, K.P., et al., The London Fibromyalgia Epidemiology Study: the prevalence of fibromyalgia syndrome in London, Ontario. J Rheumatol, 1999. 26(7): p. 1570-6.

56. Schochat, T. and C. Beckmann, [Sociodemographic characteristics, risk factors and reproductive history in subjects with fibromyalgia--results of a population-based case-control study]. Z Rheumatol, 2003. 62(1): p. 46-59. 
57. Wright, L.J., et al., Chronic pain, overweight, and obesity: findings from a community-based twin registry. J Pain, 2010. 11(7): p. 628-35.

58. Masters, E.T., et al., Electronic medical record data to identify variables associated with a fibromyalgia diagnosis: importance of health care resource utilization. J Pain Res, 2015. 8: p. 131-8.

59. Di Franco, M., et al., Misdiagnosis in fibromyalgia: a multicentre study. Clin Exp Rheumatol, 2011. 29(6 Suppl 69): p. S104-8.

\section{Chapter 3}

1. White KP, Harth M. Classification, epidemiology, and natural history of fibromyalgia. Current pain and headache reports. 2001;5(4):320-9. doi: 10.1007/s11916-001-0021-2.

2. Walitt B, Nahin RL, Katz RS, Bergman MJ, Wolfe F. The Prevalence and Characteristics of Fibromyalgia in the 2012 National Health Interview Survey. PLoS One. 2015;10(9):e0138024. doi: 10.1371/journal.pone.0138024. PubMed PMID: 26379048.

3. Clauw DJ. Fibromyalgia and related conditions. Mayo Clin Proc. 2015;90(5):680-92. doi: 10.1016/j.mayocp.2015.03.014. PubMed PMID: 25939940.

4. Giacomelli C, Talarico R, Bombardieri S, Bazzichi L. The interaction between autoimmune diseases and fibromyalgia: risk, disease course and management. Expert review of clinical immunology. 2013;9(11):1069-76. doi: 10.1586/1744666X.2013.849440. PubMed PMID: 24168413.

5. Staud R. Evidence for shared pain mechanisms in osteoarthritis, low back pain, and fibromyalgia. Current rheumatology reports. 2011;13(6):513-20. doi: 10.1007/s11926-011-0206-6. PubMed PMID: 21833699.

6. Wolfe F, Michaud K, Li T, Katz RS. Chronic conditions and health problems in rheumatic diseases: comparisons with rheumatoid arthritis, noninflammatory rheumatic disorders, systemic lupus erythematosus, and fibromyalgia. The Journal of rheumatology. 2010;37(2):305-15. doi: 10.3899/jrheum.090781. PubMed PMID: 20080915.

7. Ozgocmen S, Cimen OB, Ardicoglu O. Relationship between chest expansion and respiratory muscle strength in patients with primary fibromyalgia. Clinical rheumatology. 2002;21(1):19-22. PubMed PMID: 11954878.

8. Yang TY, Chen CS, Lin CL, Lin WM, Kuo CN, Kao CH. Risk for irritable bowel syndrome in fibromyalgia patients: a national database study. Medicine. 2015;94(10):e616. doi: 10.1097/MD.0000000000000616. PubMed PMID: 25761187.

9. Yanmaz MN, Mert M, Korkmaz M. The prevalence of fibromyalgia syndrome in a group of patients with diabetes mellitus. Rheumatol Int. 2012;32(4):871-4. doi: 10.1007/s00296-01016188. PubMed PMID: 21221595.

10. Tietjen GE, Brandes JL, Peterlin BL, Eloff A, Dafer RM, Stein MR, et al. Allodynia in migraine: association with comorbid pain conditions. Headache. 2009;49(9):1333-44. doi: 10.1111/j.15264610.2009.01521.x. PubMed PMID: 19788473.

11. Bennett RM, Jones J, Turk DC, Russell IJ, Matallana L. An internet survey of 2,596 people with fibromyalgia. BMC musculoskeletal disorders. 2007;8:27. doi: 10.1186/1471-2474-8-27. PubMed PMID: 17349056; PubMed Central PMCID: PMCPMC1829161.

12. NIH. Questions and Answers about Fibromyalgia 2014 [cited 2014 July]. Available from: http://www.niams.nih.gov/Health_Info/Fibromyalgia/default.asp. 
13. Wolfe F, Clauw Dj Fau - Fitzcharles M-A, Fitzcharles Ma Fau - Goldenberg DL, Goldenberg D1 Fau - Hauser W, Hauser W Fau - Katz RS, Katz Rs Fau - Mease P, et al. Fibromyalgia criteria and severity scales for clinical and epidemiological studies: a modification of the ACR Preliminary Diagnostic Criteria for Fibromyalgia. Arthritis Care \& Research: American College of Rheumatology; 2010. p. 600-10.

14. Choi HJ, Han JY, Seo MR, Ryu HJ, Baek HJ. Fibromyalgia with chronic rheumatic diseases in South Korea: a comparison of clinical and American College of Rheumatology criteria. International journal of rheumatic diseases. 2015. doi: 10.1111/1756-185X.12678. PubMed PMID: 25990794.

15. Wijnhoven HA, de Vet HC, Picavet HS. Explaining sex differences in chronoc musculoskeletal pain in a general population. Pain. 2006;124:158-66.

16. Williams DA, Clauw DJ. Understanding fibromyalgia: lessons from the broader pain research community. The journal of pain : official journal of the American Pain Society. 2009;10(8):77791. doi: 10.1016/j.jpain.2009.06.001. PubMed PMID: 19638325; PubMed Central PMCID:

PMC2741022.

17. CDC. Drug-poisoning Deaths Involving Opioid Analgesics: United States, 1999-2011: CDC; 2014 [cited 2015 4/17]. Available from: http://www.cdc.gov/nchs/data/databriefs/db166.htm.

18. Gaskell H, Andrew MR, Derry S, Stannard C. : Oxycodone for neuropathic pain and fibromyalgia in adults. : Cochrane Database of Systematic Reviews.

19. Fok E, Sandeman SR, Guildford AL, Martin YH. The Use of an IL-1 Receptor Antagonist Peptide to Control Inflammation in the Treatment of Corneal Limbal Epithelial Stem Cell Deficiency. Biomed Res Int. 2015;2015:516318. doi: 10.1155/2015/516318. PubMed PMID: 25705668; PubMed Central PMCID: PMC4330955.

20. Zhao Y, Sun P, Watson P, Mitchell B, Swindle R. Comparison of medication adherence and healthcare costs between duloxetine and pregabalin initiators among patients with fibromyalgia. Pain practice : the official journal of World Institute of Pain. 2011;11(3):204-16. doi: 10.1111/j.1533-2500.2010.00412.x. PubMed PMID: 20807351.

21. Andrew MR, Derry S, Aldington D, Cole P, J WP. : Amitriptyline for neuropathic pain and fibromyalgia in adults. : Cochrane Database of Systematic Reviews.

22. Administration FaD. Living with Fibromyalgia, Drugs Approved to Manage Pain Food and Drug Administration: Food and Drug Administration; 2013 [cited 2015 April 10]. Consumer Health Information]. Available from: http://www.fda.gov/downloads/ForConsumers/ConsumerUpdates/UCM107805.pdf.

23. Andrew MR, J WP, Derry S, Toelle T, C RAS. : Gabapentin for chronic neuropathic pain and fibromyalgia in adults. : Cochrane Database of Systematic Reviews.

24. Roskell NS, Beard SM, Zhao Y, Le TK. A meta-analysis of pain response in the treatment of fibromyalgia. Pain practice : the official journal of World Institute of Pain. 2011;11(6):516-27. doi: 10.1111/j.1533-2500.2010.00441.x. PubMed PMID: 21199320.

25. PT LM, AC HR, J WP. : Duloxetine for treating painful neuropathy, chronic pain or fibromyalgia. : Cochrane Database of Systematic Reviews.

26. Häuser W, Urrútia G, Tort S, Üçeyler N, Walitt B. : Serotonin and noradrenaline reuptake inhibitors (SNRIs) for fibromyalgia syndrome. : Cochrane Database of Systematic Reviews.

27. Derry S, Gill D, Phillips T, Andrew MR. : Milnacipran for neuropathic pain and fibromyalgia in adults. : Cochrane Database of Systematic Reviews.

28. J WP, Derry S, Andrew MR, Aldington D, Cole P, C RAS, et al.: Antiepileptic drugs for neuropathic pain and fibromyalgia - an overview of Cochrane reviews. : Cochrane Database of Systematic Reviews. 
29. Lanza FL, Chan FK, Quigley EM, Practice Parameters Committee of the American College of G. Guidelines for prevention of NSAID-related ulcer complications. The American journal of gastroenterology. 2009;104(3):728-38. doi: 10.1038/ajg.2009.115. PubMed PMID: 19240698.

30. NIH. Complementary, Alternative, or Integrative Health: What's In a Name? Bethesda, MD2015 [cited 2015 4/13]. Available from: https://nccih.nih.gov/health/integrative-health.

31. Rheumatology ACo. Patient Resources: Diseases and Conditions - Fibromyalgia: American College of Rheumatology; 2013 [cited 2015 April 10]. Available from:

http://www.rheumatology.org/Practice/Clinical/Patients/Diseases_And_Conditions/Fibromyalgia/

32. Ernst E. Oxford handbook of complementary medicine. Oxford ; New York: Oxford University Press; 2008. xxiii, 424 p. p.

33. Schoonees A, Visser J, Musekiwa A, Volmink J. : Pycnogenol® (extract of French maritime pine bark) for the treatment of chronic disorders. : Cochrane Database of Systematic Reviews.

34. Rao JK, Mihaliak K, Kroenke K, et al. Use of complimentary therapies for arthrtitis among patients of rheumatologists. Ann Intern Med. 1999;131(6):409-16.

35. Brown CM. Use of Alternative therapies and their impact on compliance: perceptions of community pharmacists in Texas. J Am Pharm Assoc. 1998;38(5):603-8.

36. Berman BM, Singh BB, Hartnoll SM, Singh BK, Reilly D. Primary care physicians and complementary/alternative medicine: Training, attitudes and practice patterns. J Am Board Fam Pract. 1998;11:272-81.

37. Rosenberg EI, Genao I, Chen I, Mechaber AJ, Wood JA, Faselis CJ, et al. Complementary and alternative medicine use by primary care patients with chronic pain. Pain Med. 2008;9(8):106572. doi: 10.1111/j.1526-4637.2008.00477.x. PubMed PMID: 18564996.

38. Wagner PJ, Jester D, LeClair B, et al. Taking the edge off: why patients choose St. John's Wort. J Fam Pract. 1999;48(8):615-9.

39. Barnes PM, Powell-Griner E, McFann K, Nahin RL. Complementary and alternative medicine use among adults: United States 2002. Hyattsville, MD: National Center for Health Statistics, 2004 Contract No.: Report.

40. Arcury Q, Bell \& Vitolins. Complementary and alternativemedicine use among rural adults. Complementary Health Practice Review. 2002;7(3):167-86.

41. Bausell RB, Lee WL, Berman BM. Demographic and health-related correlates to visits to complementary and alternative medical providers. Medical care. 2001;39(2):190-6. PubMed PMID: 11176556.

42. Eisenberg DM, Davis RB, Ettner SL, Appel S, Wilkey S, Van Rompay M, et al. Trends in alternative medicine use in the United States, 1990-1997: results of a follow-up of a national survey. JAMA. 1998;280:1569-75.

43. Eisenberg DM, Kessler RC, Foster C, Norlock FE, Calkins DR, Delbanco TL. Unconventional medicine in the United States: Prevalence, costs, and patterns of use. New England Journal of Medicine. 1993;328:246-52.

44. Graham RE, Ahn AC, Davis RB, O'Connor BB, Eisenberg DM, Phillips RS. Use of complementary and alternative medical therapies among racial and ethnic minority adults: results from the 2002 National Health Interview Survey. J Natl Med Assoc. 2005;97(4):535-45. PubMed PMID: 15868773; PubMed Central PMCID: PMC2568705.

45. Montalto CP, Bhargava, V., \& Hong, G. S. Use of complementary and alternative medicineby older adults: An exploratory study. Complementary Health Practice Review. 2006;11(1):27-46.

46. Tindle HA, Davis RB, Phillips RS, Eisenberg DM. Trends in use of complementary and alternative medicine by US adults: 1997-2002. Altern Ther Health Med. 2005;11(1):42-9. PubMed PMID: 15712765. 
47. Bhargava V, Hong GS, Montalto CP. Use of practitioner-based and self-care complementary and alternative medicine in the united states: A demand for health perspective. Family and Consumer Sciences Research Journal. 2012;41(1):18-35. doi: 10.1111/j.1552-3934.2012.02126.x.

48. Nahin RL, Barnes PM, Stussman BJ, Bloom B. Costs of complementary and alternative medicine (CAM) and frequency of visits to CAM practitioners: United States, 2007. National health statistics reports. 2009(18):1-14. PubMed PMID: 19771719.

49. Peregoy JA, Clarke TC, Jones LI, Stussman BJ, Nahin RL. Regional variation in use of complementary health approaches by U.S. adults. NCHS Data Brief. 2014(146):1-8. PubMed PMID: 24750666.

50. Chao MT, Wade C, Kronenberg F. Disclosure of complementary and alternative medicine to conventional medical providers: Variation by race/ethnicity and type of CAM. Journal of the National Medical Association. 2008;100(11):1341-9.

51. Upchurch DM, Chyu L, Greendale GA, Utts J, Bair YA, Zhang G, et al. Complementary and alternative medicine use among American women: Findings from the National Health Interview Survey, 2002. Journal of women's health. 2007;16(1):102-13. doi: 10.1089/jwh.2006.M074.

52. Blalock et al. Factors Associated with Potential Medication-Herb/natural Product Interactions in a Rural Community. Alternative Therapies in Health \& Medicine. 2009;15(5):26-34.

53. Nguyen LT, Davis RB, Kaptchuk TJ, Phillips RS. Use of complementary and alternative medicine and self-rated health status: Results from a national survey. Journal of General Internal Medicine. 2011;26(4):399-404. doi: 10.1007/s11606-010-1542-3.

54. Nguyen HT, Grzywacz JG, Lang W, Walkup M, Arcury TA. Effects of Complementary Therapy on Health in a National U.S. Sample of Older Adults. Journal of Alternative \& Complementary Medicine. 2010;16(7):701-6. doi: 10.1089/acm.2009.0442.

55. Neiberg RH, Aickin M, Grzywacz JG, Lang W, Quandt SA, Bell RA, et al. Occurrence and cooccurrence of types of complementary and alternative medicine use by age, gender, ethnicity, and education among adults in the United States: The 2002 national health interview survey (NHIS).

Journal of Alternative and Complementary Medicine. 2011;17(4):363-70. doi: 10.1089/acm.2009.0157.

56. Nahin RL, Dahlhamer JM, Taylor BL, Barnes PM, Stussman BJ, Simile CM, et al. Health behaviors and risk factors in those who use complementary and alternative medicine. BMC Public Health. 2007;7:217. doi: 10.1186/1471-2458-7-217. PubMed PMID: 17723149; PubMed Central PMCID: PMC2031902.

57. Nahin RL, Dahlhamer JM, Stussman BJ. Health need and the use of alternative medicine among adults who do not use conventional medicine. BMC Health Serv Res. 2010;10:220. doi: 10.1186/1472-6963-10-220. PubMed PMID: 20670418; PubMed Central PMCID: PMC2919531.

58. Misra R, Balagopal P, Klatt M, Geraghty M. Complementary and alternative medicine use among Asian Indians in the United States: A national study. Journal of Alternative and Complementary Medicine. 2010;16(8):843-52. doi: 10.1089/acm.2009.0517.

59. Mehta DH, Phillips RS, Davis RB, McCarthy EP. Use of complementary and alternative therapies by Asian Americans. Results from the National Health Interview Survey. Journal of General Internal Medicine. 2007;22(6):762-7. doi: 10.1007/s11606-007-0166-8.

60. Kennedy J, Wang CC, Wu CH. Patient Disclosure about Herb and Supplement Use among Adults in the US. Evidence-based complementary and alternative medicine : eCAM. 2008;5(4):451-6. doi: 10.1093/ecam/nem045. PubMed PMID: 18955213; PubMed Central PMCID: PMC2586312. 61. Kannan VD, Gaydos LM, Atherly AJ, Druss BG. Medical utilization among wellness consumers. Med Care Res Rev. 2010;67(6):722-36. Epub 2010/06/04. doi: 10.1177/1077558710370706. PubMed PMID: 20519427. 
62. Jones L, Sciamanna C, Lehman E. Are those who use specific complementary and alternative medicine therapies less likely to be immunized? Preventive medicine. 2010;50(3):148-54. doi: 10.1016/j.ypmed.2009.12.001.

63. Barner JC, Bohman TM, Brown CM, Richards KM. Use of complementary and alternative medicine for treatment among African-Americans: A multivariate analysis. Research in Social and Administrative Pharmacy. 2010;6(3):196-208. doi: 10.1016/j.sapharm.2009.08.001.

64. Hildreth KD EC. Alternative worldviews and the utilization of conventional and complementary medicine. Sociological Inquiry. 2007;77(1):76-103.

65. Birdee GS, Phillips RS, Davis RB, Gardiner P. Factors associated with pediatric use of complementary and alternative medicine. Pediatrics. 2010;125(2):249-56. doi: 10.1542/peds.2009-1406.

66. Grzywacz JG, Lang W, Suerken C, Quandt SA, Bell RA, Arcury TA. Age, race, and ethnicity in the use of complementary and alternative medicine for health self-management: evidence from the 2002 National Health Interview Survey. Journal of aging and health. 2005;17(5):547-72. Epub 2005/09/24. doi: 10.1177/0898264305279821. PubMed PMID: 16177450.

67. Grzywacz JG, Suerken CK, Neiberg RH, Lang W, Bell RA, Quandt SA, et al. Age, ethnicity, and use of complementary and alternative medicine in health self-management. Journal of health and social behavior. 2007;48(1):84-98. PubMed PMID: 17476925 .

68. Gardiner et al. Factors Associated with Herbal Therapy use by Adults in the United States. Alternative Therapies in Health \& Medicine. 2007;13(2):22-9.

69. Elmer GW, Lafferty WE, Tyree PT, Lind BK. Potential interactions between complementary/alternative products and conventional medicines in a Medicare population. Ann Pharmacother. 2007;41(10):1617-24. Epub 2007/09/06. doi: 10.1345/aph.1K221. PubMed PMID: 17785609; PubMed Central PMCID: PMCPMC2864004.

70. Ellison CG, Bradshaw M, Roberts CA. Spiritual and religious identities predict the use of complementary and alternative medicine among US adults. Preventive medicine. 2012;54(1):912. doi: 10.1016/j.ypmed.2011.08.029.

71. Upchurch DM, Dye CE, Chyu L, Gold EB, Greendale GA. Demographic, behavioral, and health correlates of complementary and alternative medicine and prayer use among midlife women: 2002. Journal of women's health. 2010;19(1):23-30. doi: 10.1089/jwh.2008.1096. PubMed PMID: 20088655; PubMed Central PMCID: PMC2828262.

72. Davis MA, West AN, Weeks WB, Sirovich BE. Health Behaviors and Utilization among Users of Complementary and Alternative Medicine for Treatment versus Health Promotion. Health services research. 2011;46(5):1402-16. doi: 10.1111/j.1475-6773.2011.01270.x.

73. Barnes PM, Bloom B, Nahin RL. Complementary and alternative medicine use among adults and children: United States, 2007. National health statistics reports. 2008(12):1-23. Epub 2009/04/14. PubMed PMID: 19361005.

74. Bardia A, Nisly NL, Zimmerman MB, Gryzlak BM, Wallace RB. Use of herbs among adults based on evidence-based indications: findings from the National Health Interview Survey. Mayo Clin Proc. 2007;82(5):561-6. Epub 2007/05/12. doi: 10.4065/82.5.561. PubMed PMID: 17493422; PubMed Central PMCID: PMCPMC1964882.

75. Arcury TA, Grzywacz JG, Bell RA, Neiberg RJ, Lang W, Quandt SA. Herbal remedy use as health self-management among older adults. Journals of Gerontology: Series B: Psychological Sciences and Social Sciences. 2007;62B(2):S142-S9.

76. Tom Xu K, Farrell TW. The complementarity and substitution between unconventional and mainstream medicine among racial and ethnic groups in the United States. Health Serv Res. 2007;42(2):811-26. Epub 2007/03/17. doi: 10.1111/j.1475-6773.2006.00628.x. PubMed PMID: 17362219; PubMed Central PMCID: PMCPMC1955362. 
77. Brown CM, Barner JC, Richards KM, Bohman TM. Patterns of complementary and alternative medicine use in African Americans. Journal of Alternative and Complementary Medicine. 2007;13(7):751-8. doi: 10.1089/acm.2006.6392.

78. Sawni A, Thomas R. Pediatricians' attitudes, experience and referral patterns regarding Complementary/Alternative Medicine: a national survey. BMC Complement Altern Med. 2007;7:18. doi: 10.1186/1472-6882-7-18. PubMed PMID: 17547752; PubMed Central PMCID: PMC1894987.

79. Mehta DH, Gardiner PM, Phillips RS, McCarthy EP. Herbal and dietary supplement disclosure to health care providers by individuals with chronic conditions. J Altern Complement Med. 2008;14(10):1263-9. Epub 2008/11/27. doi: 10.1089/acm.2008.0290. PubMed PMID: 19032071; PubMed Central PMCID: PMCPMC2787410.

80. Organization WH. WHO Monographs on Slected Medicinal Plants. 2003.

81. Carle R GK. Chamomile: a pharmacological and clinical profile. Drugs of Today. 1992;28:55965.

82. Gould L RC, Gomprecht RF. Cardiac effect of chamomile tea. Journal of clinical pharmacology. 1973;13:475-9.

83. Mascolo N, Autore G, Capasso F, Menghini A, Fasulo MP. - Biological screening of Italian medicinal plants for anti-inflammatory activity. 1987;- 1(- 1):- 31 .

84. al. TBe. Taraxacum offi cinale W.: Pharmacological effect of an ethanol extract. Pharmacology Research. 1993;27(Suppl.1):23-4.

85. World Health Organization. WHO monographs on selected medicinal plants. Geneva: World Health Organization; 1999-2009. v. <1-4> p.

86. Yasukawa K, Yamaguchi A, Arita J, Sakurai S, Ikeda A, Takido M. - Inhibitory effect of edible plant extracts on 12-O-tetradecanoylphorbol-13-acetate-induced ear oedema in mice. 1993;- 7(2):- 189.

87. Delaveau P, Lallouette P, Tessier AM. [Stimulation of the phagocytic activity of R.E.S. by plant extracts (author's transl)]. Planta medica. 1980;40(1):49-54. doi: 10.1055/s-2008-1074941. PubMed PMID: 6999511.

88. Yesilada E, Ustun O, Sezik E, Takaishi Y, Ono Y, Honda G. Inhibitory effects of Turkish folk remedies on inflammatory cytokines: interleukin-1alpha, interleukin-1beta and tumor necrosis factor alpha. J Ethnopharmacol. 1997;58(1):59-73. PubMed PMID: 9324006.

89. al. WHe. In vitro inhibition of prostaglandin biosynthesis by essential oils and phenolic compounds. Plant Medica. 1986;3:184-7.

90. Yasukawa K, Takeuchi M, Takido M. Humulon, a bitter in the hop, inhibits tumor promotion by 12-O-tetradecanoylphorbol-13-acetate in two-stage carcinogenesis in mouse skin. Oncology. 1995;52(2):156-8. PubMed PMID: 7854777.

91. H. H. Economic and medicinal plant research. Wagner H HH, Farnsworth NR, editor. London: Academic Press; 1985.

92. Duwiejua M, Zeitlin IJ, Waterman PG, Gray AI. Anti-inflammatory activity of Polygonum bistorta, Guaiacum officinale and Hamamelis virginiana in rats. The Journal of pharmacy and pharmacology. 1994;46(4):286-90. PubMed PMID: 8051612.

93. Cuellar MJ, Giner RM, Recio MC, Manez S, Rios JL. Topical anti-inflammatory activity of some Asian medicinal plants used in dermatological disorders. Fitoterapia. 2001;72(3):221-9. PubMed PMID: 11295297.

94. Butenko IG, Gladtchenko SV, Galushko SV. Anti-inflammatory properties and inhibition of leukotriene $\mathrm{C} 4$ biosynthesis in vitro by flavonoid baicalein from Scutellaria baicalensis georgy roots. Agents and actions. 1993;39 Spec No:C49-51. PubMed PMID: 8273583.

95. Kimura Y, Yokoi K, Matsushita N, Okuda H. Effects of flavonoids isolated from scutellariae radix on the production of tissue-type plasminogen activator and plasminogen activator inhibitor1 
induced by thrombin and thrombin receptor agonist peptide in cultured human umbilical vein endothelial cells. The Journal of pharmacy and pharmacology. 1997;49(8):816-22. Epub 1997/08/01. PubMed PMID: 9379363.

96. al. URe. American herbal pharmacopeia. Santa Cruz, CA: American Herbal Pharmacopeia; 1999.

97. Wagner I, Greim C, Laufer S, Heide L, Gleiter CH. Influence of willow bark extract on cyclooxygenase activity and on tumor necrosis factor alpha or interleukin 1 beta release in vitro and ex vivo. Clin Pharmacol Ther. 2003;73(3):272-4. Epub 2003/03/07. doi: 10.1067/mcp.2003.32. PubMed PMID: 12621392.

98. Chung CP, Park JB, Bae KH. Pharmacological effects of methanolic extract from the root of Scutellaria baicalensis and its flavonoids on human gingival fibroblast. Planta medica. 1995;61(2):150-3. Epub 1995/04/01. doi: 10.1055/s-2006-958036. PubMed PMID: 7753922.

99. Cheng GF, Liu DP, Yang DX, He KQ, Bai JY, Zhu XY. Antiinflammatory effects of Tremulacin, a Salicin-related substance isolated from Populus tomentosa Carr. leaves. Phytomedicine. 1994;1(3):209-11. doi: 10.1016/S0944-7113(11)80067-X. PubMed PMID: 23195941.

100. Srivastava KC, Mustafa T. Ginger (Zingiber officinale) in rheumatism and musculoskeletal disorders. Medical hypotheses.39(4):342-8. doi: 10.1016/0306-9877(92)90059-L.

101. Srivastava KC. Aqueous extracts of onion, garlic and ginger inhibit platelet aggregation and alter arachidonic acid metabolism. Biomed Biochim Acta. 1984;43(8-9):S335-46. PubMed PMID: 6440548.

102. Mustafa T SK, Jensen KB. Drug development report 9. Pharmacology of ginger, Zingiber officinale. Journal of Drug Development. 1993;6:25-39.

103. Suekawa M, Yuasa K, Isono M, Sone H, Ikeya Y, Sakakibara I, et al. [Pharmacological studies on ginger. IV. Effect of (6)-shogaol on the arachidonic cascade]. Nihon yakurigaku zasshi Folia pharmacologica Japonica. 1986;88(4):263-9. Epub 1986/10/01. PubMed PMID: 3098654.

104. Sharma JN, Srivastava KC, Gan EK. Suppressive effects of eugenol and ginger oil on arthritic rats. Pharmacology. 1994;49(5):314-8. PubMed PMID: 7862743.

105. Shunro K, Morimitsu Y, Toshihiko O. Chemistry of Ginger Components and Inhibitory Factors of the Arachidonic Acid Cascade. Food Phytochemicals for Cancer Prevention II. ACS Symposium Series. 547: American Chemical Society; 1994. p. 244-50.

106. Wang M, Guilbert LJ, Ling L, Li J, Wu Y, Xu S, et al. Immunomodulating activity of CVTE002, a proprietary extract from North American ginseng (Panax quinquefolium). The Journal of pharmacy and pharmacology. 2001;53(11):1515-23. PubMed PMID: 11732754.

107. Assinewe VA, Amason JT, Aubry A, Mullin J, Lemaire I. Extractable polysaccharides of Panax quinquefolius L. (North American ginseng) root stimulate TNFalpha production by alveolar macrophages. Phytomedicine. 2002;9(5):398-404. PubMed PMID: 12222658.

108. Audette JF, Bailey A. Integrative pain medicine : the science and practice of complementary and alternative medicine in pain management. Totowa, N.J.: Humana; 2008. xiv, 579 p. p.

109. NIH. Thunder God Vine 2012 [cited 2015 May 23]. Available from: https://nccih.nih.gov/health/tgvine.

110. GB L. A pharmacometric evaluation of nine Bio-Strath herbal remedies. Medita. 1978;8:3-19.

111. al CBe. Pharmacological properties of saponin fractions obtained from domestic crude drugs: Saponaria officinalis, Primula officinalis and Aesculus hippocastanum. Herba Polonica. 1976;22:154-62.

112. Cabrera C. Fibromyalgia : a journey toward healing. Chicago: Contemporary Books; 2002. xiii, 318 p. p.

113. Shelton F. Pioneer comforts and kitchen remedies;: Oldtimey highland secrets from the Blue Ridge and Great Smoky Mountains: Hutcraft; 1965. 24 p.

114. Bolyard JL. Medicinal plants and home remedies of Appalachia. Springfield, Ill.: Thomas; 1981. xvii, 187 p. p. 
115. Crellin JK, Philpott J, Bass ALT. Herbal medicine past and present. Durham: Duke University Press; 1990.

116. Amjad H. Folk Medicine of Appalachia: A Vanishing Tradition: lulu.com (self-published); 2006. $340 \mathrm{p}$.

117. Krochmal A, Walters RS, Doughty RM. A guide to medicinal plants of Appalachia. Washington,: U.S. Forest Service; for sale by the Supt. of Docs.; 1971. 291 p. p.

118. Collins KC, Hunter L. Foxfire 11 : the old homeplace, wild plant uses, preserving and cooking food, hunting stories, fishing, and more affairs of plain living. 1st ed. New York: Anchor Books; 1999. xi, 313 p. p.

119. Stephenson SL. A natural history of the central Appalachians. 1st ed. Morgantown: West Virginia University Press; 2013. xi, 259 p. p.

120. Crellin JK, Bass ALT, Philpott J. Trying to give ease : Tommie Bass and the story of herbal medicine. Durham: Duke University Press; 1997. xii. 335 p. p.

121. Wilson CR, Ferris WR. Encyclopedia of Southern culture. Chapel Hill: University of North Carolina Press; 1989. xxi, 1634 p. p.

122. Goodwin JG. Going 'Senging. Goldenseal. 1985:16-21.

123. Bennett TGaA. Catfish: Portrait of an Herb Doctor. Goldenseal. 1977:46-51.

124. Brunvand JH. American folklore : an encyclopedia. New York: Garland Pub.; 1996. xviii, 794 p. p.

125. Braverman DL, Ericken JJ, Shah RV, Franklin DJ. Interventions in chronic pain management 3. New frontiers in pain management: complementary techniques. Arch Phys Med Rehabil. 2003;84(3 Suppl 1):S45-9.

126. O'Neil M, Hannah KL. Understanding the cultures of Prescription Drug Abuse, Misuse, Addiction, and Diversion. WV Medical Journal. 2010;106(4):64-70.

127. Fleming S RDPMMPFMF. CAM therapies among primary care patients using opioid therapy for chronic pain. BMC Complementary and Alternative Medicine. 2007;7(15).

128. Ernst E. Usage of complementary therapies in rheumatology: a systematic review. Clinical rheumatology. 1998;17(4):301-5. PubMed PMID: 9776112.

129. Pullar T, Capell HA, Millar A, Brooks RG. Alternative medicine: cost and subjective benefit in rheumatoid arthritis. British medical journal. 1982;285(6355):1629-31. PubMed PMID: 6814682; PubMed Central PMCID: PMC1500773.

130. Ernst E, White A. The BBC survey of complementary medicine use in the UK. Complementary therapies in medicine. 2000;8(1):32-6. doi: http://dx.doi.org/10.1016/S0965-2299(00)90833-1.

131. Higham C, Ashcroft C, Jayson MI. Non-prescribed treatments in rheumatic diseases. The Practitioner. 1983;227(1381):1201-5. PubMed PMID: 6604270.

132. Dimmock S, Troughton PR, Bird HA. Factors predisposing to the resort of complementary therapies in patients with fibromyalgia. Clinical rheumatology. 1996;15(5):478-82. PubMed PMID: 8894361.

133. Shaver JL, Wilbur J, Lee H, Robinson FP, Wang E. Self-reported medication and herb/supplement use by women with and without fibromyalgia. Journal of women's health. 2009;18(5):709-16. doi: 10.1089/jwh.2008.1194. PubMed PMID: 19445618.

134. Prevention CfDCa. NHIS Survey Description. In: Statistics DoHISNCfH, editor. Hyattsville, MDDecember 2003.

135. Centers for Disease Control and Prevention USDoHaHS. 2012 National Health Interview Survey (NHIS) Public Use Data Release. In: Statistics DoHI, Statistics NCfH, editors. Hyattsville, Maryland: Centers for Disease Control and Prevention

U.S. Department of Health and Human Services; 2013.

136. Ursini F, Naty S, Grembiale R. Fibromyalgia and obesity: the hidden link. Rheumatol Int. 2011;31(11):1403-8. doi: 10.1007/s00296-011-1885-z. 
137. NIH. Classification of Overweight and Obesity by BMI, Waist Circumference, and Associated Disease Risks: National Heart, Lung, and Blood Institute [cited 2015 February 7]. Available from:

https://www.nhlbi.nih.gov/health/educational/lose_wt/BMI/bmi_dis.htm.

138. FDA. FDA Approves First Drug for Treating Fibromyalgia: U.S. Department of Health and Human Services; 2007. Available from: http://www.fda.gov/NewsEvents/Newsroom/PressAnnouncements/2007/ucm108936.htm.

139. Choy E, Perrot S, Leon T, Kaplan J, Petersel D, Ginovker A, et al. A patient survey of the impact of fibromyalgia and the journey to diagnosis. BMC Health Services Research. 2010;10(1):1-9. doi: 10.1186/1472-6963-10-102.

\section{Chapter 4}

1. Cauffield JS. The Psychoscoial aspects of complementary and alternative medicine. Pharmacotherapy. 2000;20(11):1289-94.

2. Manchikanti L, Singh V, Datta S, Cohen SP, Hirsch JA. American Society of Interventional Pain Physicians. Comprehensive review of epidemiology, scope, and impact of spinal pain. Pain Physician. 2009;12:E35-70.

3. Andersson HI. The epidemiology of chronic pain in a Swedish rural area. Qual Life Res. 1994;3:s19-s26.

4. Classification of chronic pain. Descriptions of chronic pain syndromes and definitions of pain terms. Prepared by the International Association for the Study of Pain, Subcommittee on Taxonomy. Pain Supplement. 1986;3:S1-226. PubMed PMID: 3461421.

5. Brattberg G, Thorslund M, Wikman A. The prevalence of pain in a general population. The results of a postal survey in a county of Sweden. Pain. 1989;37:215-22.

6. Wijnhoven HA, de Vet HC, Picavet HS. Explaining sex differences in chronoc musculoskeletal pain in a general population. Pain. 2006;124:158-66.

7. Gentile DA, Woodhouse J, Lynch P, Maier J, McJunkin T. Reliability and Validity of the Global Pain Scale with Chronic Pain Sufferers. Pain Physician. 2011;14:61-70.

8. Tyson Sf BP. How to measure pain in neurological conditions? A systematic review of psychometric properties and clinical utility of measurement tools. Clinical Rehabilitation. 2013.

9. Singh JA, Luo R Fau - Landon GC, Landon Gc Fau - Suarez-Almazor M, Suarez-Almazor M. Reliability and Clinically Important Improvement Thresholds for Osteoarthritis Pain and Function Scales: A Multicenter Study.

10. Salaffi F, Sarzi-Puttini P, Ciapetti A, Atzeni F. Assessment instruments for patients with fibromyalgia: properties, applications and interpretation. Clinical and experimental rheumatology. 2009;27(5 Suppl 56):S92-105. PubMed PMID: 20074447.

11. Reed MD, Van Nostran W. Assessing pain intensity with the visual analogue scale: A plea for uniformity. The Journal of Clinical Pharmacology. 2013:n/a-n/a. doi: 10.1002/jcph.250.

12. Plan EL, Elshoff Jp Fau - Stockis A, Stockis A Fau - Sargentini-Maier ML, Sargentini-Maier Ml Fau - Karlsson MO, Karlsson MO. Likert pain score modeling: a Markov integer model and an autoregressive continuous model.

13. Lukas A, Barber Jb Fau - Johnson P, Johnson P Fau - Gibson SJ, Gibson SJ. Observer-rated pain assessment instruments improve both the detection of pain and the evaluation of pain intensity in people with dementia.

14. Liu JYW, Briggs M, Closs SJ. The Psychometric Qualities of Four Observational Pain Tools 
(OPTs) for the Assessment of Pain in Elderly People with Osteoarthritic Pain. Journal of pain and symptom management. 2010;40(4):582-98. doi:

http://dx.doi.org/10.1016/j.jpainsymman.2010.02.022.

15. Hawker GA, Mian S, Kendzerska T, French M. Measures of adult pain: Visual Analog Scale for Pain (VAS Pain), Numeric Rating Scale for Pain (NRS Pain), McGill Pain Questionnaire (MPQ), Short-Form McGill Pain Questionnaire (SF-MPQ), Chronic Pain Grade Scale (CPGS), Short Form-36 Bodily Pain Scale (SF-36 BPS), and Measure of Intermittent and Constant Osteoarthritis Pain (ICOAP). Arthritis Care \& Research. 2011;63(S11):S240-S52. doi: 10.1002/acr.20543.

16. Gelinas C, Puntillo Ka Fau - Joffe AM, Joffe Am Fau - Barr J, Barr J. A validated approach to evaluating psychometric properties of pain assessment tools for use in nonverbal critically ill adults.

17. Myers KA, Bello-Espinosa LE, Kherani A, Wei XC, Innes AM. TUBA1A Mutation Associated With Eye Abnormalities in Addition to Brain Malformation. Pediatr Neurol. 2015. doi: 10.1016/j.pediatrneurol.2015.07.004. PubMed PMID: 26294046.

18. Gendreau M, Hufford MR, Stone AA. Measuring clinical pain in chronic widespread pain: selected methodological issues. Chronic Generalised Musculoskeletal Pain. 2003;17(4):575-92. doi: http://dx.doi.org/10.1016/S1521-6942(03)00031-7.

19. Carter GT, Duong V, Ho S, Ngo KC, Greer CL, Weeks DL. Side effects of commonly prescribed analgesic medications. Physical medicine and rehabilitation clinics of North America. 2014;25(2):457-70. doi: 10.1016/j.pmr.2014.01.007. PubMed PMID: 24787343.

20. Devor M. - Neuropathic pain: what do we do with all these theories? 2001;- 45(- 9):- 1127.

21. Lipnik-Stangelj M. Mediators of inflammation as targets for chronic pain treatment. Mediators of inflammation. 2013;2013:783235. doi: 10.1155/2013/783235. PubMed PMID: 24347834; PubMed Central PMCID: PMC3848385.

22. Lanza FL, Chan FK, Quigley EM, Practice Parameters Committee of the American College of G. Guidelines for prevention of NSAID-related ulcer complications. The American journal of gastroenterology. 2009;104(3):728-38. doi: 10.1038/ajg.2009.115. PubMed PMID: 19240698.

23. Williams DA, Clauw DJ. Understanding fibromyalgia: lessons from the broader pain research community. The journal of pain : official journal of the American Pain Society. 2009;10(8):77791. doi: 10.1016/j.jpain.2009.06.001. PubMed PMID: 19638325; PubMed Central PMCID: PMC2741022.

24. Painter JT, Crofford LJ. Chronic opioid use in fibromyalgia syndrome: a clinical review. Journal of clinical rheumatology : practical reports on rheumatic \& musculoskeletal diseases.

2013;19(2):72-7. doi: 10.1097/RHU.0b013e3182863447. PubMed PMID: 23364665.

25. Gaskell H, Andrew MR, Derry S, Stannard C. : Oxycodone for neuropathic pain and fibromyalgia in adults. : Cochrane Database of Systematic Reviews.

26. Fok E, Sandeman SR, Guildford AL, Martin YH. The Use of an IL-1 Receptor Antagonist Peptide to Control Inflammation in the Treatment of Corneal Limbal Epithelial Stem Cell Deficiency. Biomed Res Int. 2015;2015:516318. doi: 10.1155/2015/516318. PubMed PMID: 25705668; PubMed Central PMCID: PMC4330955.

27. CDC. Drug-poisoning Deaths Involving Opioid Analgesics:

United States, 1999-2011: CDC; 2014 [cited 2015 4/17]. Available from:

http://www.cdc.gov/nchs/data/databriefs/db166.htm.

28. O'Neil M, Hannah KL. Understanding the cultures of Prescription Drug Abuse, Misuse, Addiction, and Diversion. WV Medical Journal. 2010;106(4):64-70.

29. Centers for Disease Control and Prevention USDoHaHS. Drug Overdose Deaths by State, US 2013 and 20142015 [updated Dec 18, 2015]. Available from:

http://www.cdc.gov/drugoverdose/data/statedeaths.html. 
30. Wagner PJ, Jester D, LeClair B, et al. Taking the edge off: why patients choose St. John's Wort. J Fam Pract. 1999;48(8):615-9.

31. Eisenberg DM, Davis RB, Ettner SL, Appel S, Wilkey S, Van Rompay M, et al. Trends in alternative medicine use in the United States, 1990-1997: results of a follow-up of a national survey. JAMA. 1998;280:1569-75.

32. Braverman DL, Ericken JJ, Shah RV, Franklin DJ. Interventions in chronic pain management 3. New frontiers in pain management: complementary techniques. Arch Phys Med Rehabil. 2003;84(3 Suppl 1):S45-9.

33. Rosenberg EI, Genao I, Chen I, Mechaber AJ, Wood JA, Faselis CJ, et al. Complementary and alternative medicine use by primary care patients with chronic pain. Pain Med. 2008;9(8):106572. doi: 10.1111/j.1526-4637.2008.00477.x. PubMed PMID: 18564996.

34. Fleming S RDPMMPFMF. CAM therapies among primary care patients using opioid therapy for chronic pain. BMC Complementary and Alternative Medicine. 2007;7(15).

35. Rosenberg Ei GICIMAJWJAFCJKJMMORJPMP. Complementary and Alternative Medicine Use by Primary Care Patients with Chronic pain. Pain medicine (Malden, Mass) JID 100894201. 2008;9(8):1065-72.

36. Eisenberg DM, Kessler RC, Foster C, Norlock FE, Calkins DR, Delbanco TL. Unconventional medicine in the United States: Prevalence, costs, and patterns of use. New England Journal of Medicine. 1993;328:246-52.

37. Druss BG, Rosenheck RA. Association between use of unconventional therapies and conventional medical services. JAMA. 1999;282(7):651-6. PubMed PMID: 10517718.

38. Rao JK, Mihaliak K, Kroenke K, et al. Use of complimentary therapies for arthrtitis among patients of rheumatologists. Ann Intern Med. 1999;131(6):409-16.

39. Brown CM. Use of Alternative therapies and their impact on compliance: perceptions of community pharmacists in Texas. J Am Pharm Assoc. 1998;38(5):603-8.

40. Nahin RL, Barnes PM, Stussman BJ, Bloom B. Costs of complementary and alternative medicine (CAM) and frequency of visits to CAM practitioners: United States, 2007. National health statistics reports. 2009(18):1-14. PubMed PMID: 19771719.

41. Herman PM, Poindexter BL, Witt CM, Eisenberg DM. Are complementary therapies and integrative care cost-effective? A systematic review of economic evaluations. BMJ open. 2012;2(5). doi: 10.1136/bmjopen-2012-001046. PubMed PMID: 22945962; PubMed Central PMCID: PMCPMC3437424.

42. Santaguida P, Gross A, United States. Agency for Healthcare Research and Quality., McMaster University. Evidence-based Practice Center. Complementary and alternative medicine in back pain utilization report. Rockville, MD: Agency for Healthcare Research and Quality; 2009. ix, 149 p. p.

43. NIH. Complementary, Alternative, or Integrative Health: What's In a Name? Bethesda, MD2015 [cited 2015 4/13]. Available from: https://nccih.nih.gov/health/integrative-health.

44. Cabrera C. Fibromyalgia : a journey toward healing. Chicago: Contemporary Books; 2002. xiii, 318 p. p.

45. Shelton F. Pioneer comforts and kitchen remedies;: Oldtimey highland secrets from the Blue Ridge and Great Smoky Mountains: Hutcraft; 1965. 24 p.

46. Bolyard JL. Medicinal plants and home remedies of Appalachia. Springfield, Ill.: Thomas; 1981. xvii, 187 p. p.

47. Crellin JK, Philpott J, Bass ALT. Herbal medicine past and present. Durham: Duke University Press; 1990.

48. Amjad H. Folk Medicine of Appalachia: A Vanishing Tradition: lulu.com (self-published); 2006. $340 \mathrm{p}$. 
49. Krochmal A, Walters RS, Doughty RM. A guide to medicinal plants of Appalachia. Washington,: U.S. Forest Service; for sale by the Supt. of Docs.; 1971. 291 p. p.

50. Collins KC, Hunter L. Foxfire 11 : the old homeplace, wild plant uses, preserving and cooking food, hunting stories, fishing, and more affairs of plain living. 1st ed. New York: Anchor Books; 1999. xi, 313 p. p.

51. Stephenson SL. A natural history of the central Appalachians. 1st ed. Morgantown: West Virginia University Press; 2013. xi, 259 p. p.

52. Audette JF, Bailey A. Integrative pain medicine : the science and practice of complementary and alternative medicine in pain management. Totowa, N.J.: Humana; 2008. xiv, 579 p. p.

53. Mason L, Moore RA, Derry S, Edwards JE, McQuay HJ. Systematic review of topical capsaicin for the treatment of chronic pain. BMJ. 2004;328(7446):991. doi:

10.1136/bmj.38042.506748.EE. PubMed PMID: 15033881; PubMed Central PMCID: PMC404499.

54. Pullar T, Capell HA, Millar A, Brooks RG. Alternative medicine: cost and subjective benefit in rheumatoid arthritis. British medical journal. 1982;285(6355):1629-31. PubMed PMID: 6814682; PubMed Central PMCID: PMC1500773.

55. Ernst E, White A. The BBC survey of complementary medicine use in the UK. Complementary therapies in medicine. 2000;8(1):32-6. doi: http://dx.doi.org/10.1016/S0965-2299(00)90833-1.

56. Higham C, Ashcroft C, Jayson MI. Non-prescribed treatments in rheumatic diseases. The Practitioner. 1983;227(1381):1201-5. PubMed PMID: 6604270.

57. Dimmock S, Troughton PR, Bird HA. Factors predisposing to the resort of complementary therapies in patients with fibromyalgia. Clinical rheumatology. 1996;15(5):478-82. PubMed PMID: 8894361.

58. Cavender A. Folk Medicine in Southern Appalachia: The University of North Carolina Press; 2003. $266 \mathrm{p}$.

59. Crellin JK, Bass ALT, Philpott J. Trying to give ease : Tommie Bass and the story of herbal medicine. Durham: Duke University Press; 1997. xii. 335 p. p.

60. Wilson CR, Ferris WR. Encyclopedia of Southern culture. Chapel Hill: University of North Carolina Press; 1989. xxi, 1634 p. p.

61. Goodwin JG. Going 'Senging. Goldenseal. 1985:16-21.

62. Bennett TGaA. Catfish: Portrait of an Herb Doctor. Goldenseal. 1977:46-51.

63. Brunvand JH. American folklore : an encyclopedia. New York: Garland Pub.; 1996. xviii, 794 p. p.

64. Weinstein ND, Rothman AJ, Sutton SR. Stage theories of health behavior: conceptual and methodological issues. Health Psychology. 1998;17(3):290-9.

65. Prochaska JO, DiClemente CC, Norcross JC. In search of how people changw: applications to addictive behaviors. American Psychologist. 1992;47(9):1102-14.

66. Prochaska JO, Norcross JC, DiClemente CC. Changing for good : the revolutionary program that explains the six stages of change and teaches you how to free yourself from bad habits. 1st ed. New York: W. Morrow; 1994. 304 p. p.

67. Prochaska JO. Decision making in the transtheoretical model for behavior change. . Medical Decision Making. 2008;28(6):845-9.

68. Di Noia J, Prochaska JO. Dietary stages of change and decisional balance: a meta-analytic review. American Journal of Health Behavior. 2010;34(5):618-32.

69. Plotnikoff GA, Quigley JM. Prevalence of Severe Hypovitaminosis D in Patients With Persistent, Nonspecific Musculoskeletal Pain. Mayo Clinic proceedings. 2003;78(12):1463-70. doi: http://dx.doi.org/10.4065/78.12.1463. 
70. Jezewski MA, Finnell DS, Wu Y-WB, Meeker MA, Sessanna L, Lee J. Psychometric testing of four transtheoretical model questionnaires for the behavior, completing health care proxies.

Research in nursing \& health. 2009;32(6):606-20. doi: 10.1002/nur.20352.

71. Henchoz Y, Zufferey P, So A. Stages of change, barriers, benefits, and preferences for exercise in RA patients: a cross-sectional study. Scand J Rheumatol. 2013;42(2):136-45. doi:

10.3109/03009742.2012.724707. PubMed PMID: 23244196.

72. Johnson P, Fallon Ea Fau - Harris BS, Harris Bs Fau - Burton B, Burton B. Body satisfaction is associated with Transtheoretical Model constructs for physical activity behavior change.

73. Choi JH, Chung Km Fau - Park K, Park K. Psychosocial predictors of four health-promoting behaviors for cancer prevention using the stage of change of Transtheoretical Model. LID 10.1002/pon.3278 [doi].

74. Glanz K, Rimer B, Viswanath K. Health Behavior and Health Education: Theory, Research, and Practice. San Francisco, CA: Jossey-Bass; 2008.

75. Plotnikoff RC, Lippke S, Johnson ST, Hotz SB, Birkett NJ, Rossi SR. Applying the stages of change to multiple low-fat dietary behavioral contexts. An examination of stage occupation and discontinuity. Appetite. 2009;53(3):345-53. doi: http://dx.doi.org.www.libproxy.wvu.edu/10.1016/j.appet.2009.07.016.

76. NIH. Classification of Overweight and Obesity by BMI, Waist Circumference, and Associated Disease Risks: National Heart, Lung, and Blood Institute [cited 2015 February 7]. Available from:

https://www.nhlbi.nih.gov/health/educational/lose wt/BMI/bmi dis.htm.

77. Neiberg RH, Aickin M, Grzywacz JG, Lang W, Quandt SA, Bell RA, et al. Occurrence and cooccurrence of types of complementary and alternative medicine use by age, gender, ethnicity, and education among adults in the United States: The 2002 national health interview survey (NHIS).

Journal of Alternative and Complementary Medicine. 2011;17(4):363-70. doi: 10.1089/acm.2009.0157.

78. Wu CH, Wang CC, Tsai MT, Huang WT, Kennedy J. Trend and pattern of herb and supplement use in the United States: results from the 2002, 2007, and 2012 national health interview surveys. Evidence-based complementary and alternative medicine : eCAM. 2014;2014:872320. doi: 10.1155/2014/872320. PubMed PMID: 25574184; PubMed Central PMCID: PMC4276694.

79. Shaver JL, Wilbur J, Lee H, Robinson FP, Wang E. Self-reported medication and herb/supplement use by women with and without fibromyalgia. Journal of women's health. 2009;18(5):709-16. doi: 10.1089/jwh.2008.1194. PubMed PMID: 19445618.

80. Mehta DH, Gardiner PM, Phillips RS, McCarthy EP. Herbal and dietary supplement disclosure to health care providers by individuals with chronic conditions. J Altern Complement Med. 2008;14(10):1263-9. Epub 2008/11/27. doi: 10.1089/acm.2008.0290. PubMed PMID: 19032071; PubMed Central PMCID: PMCPMC2787410.

81. Barnes PM, Bloom B, Nahin RL. Complementary and alternative medicine use among adults and children: United States, 2007. National health statistics reports. 2008(12):1-23. Epub 2009/04/14. PubMed PMID: 19361005.

82. Ellison CG, Bradshaw M, Roberts CA. Spiritual and religious identities predict the use of complementary and alternative medicine among US adults. Preventive medicine. 2012;54(1):912. doi: 10.1016/j.ypmed.2011.08.029.

83. Grzywacz JG, Suerken CK, Neiberg RH, Lang W, Bell RA, Quandt SA, et al. Age, ethnicity, and use of complementary and alternative medicine in health self-management. Journal of health and social behavior. 2007;48(1):84-98. PubMed PMID: 17476925.

84. Grzywacz JG, Lang W, Suerken C, Quandt SA, Bell RA, Arcury TA. Age, race, and ethnicity in the use of complementary and alternative medicine for health self-management: evidence from 
the 2002 National Health Interview Survey. Journal of aging and health. 2005;17(5):547-72. Epub 2005/09/24. doi: 10.1177/0898264305279821. PubMed PMID: 16177450.

85. Misra R, Balagopal P, Klatt M, Geraghty M. Complementary and alternative medicine use among Asian Indians in the United States: A national study. Journal of Alternative and Complementary Medicine. 2010;16(8):843-52. doi: 10.1089/acm.2009.0517.

86. Nahin RL, Dahlhamer JM, Stussman BJ. Health need and the use of alternative medicine among adults who do not use conventional medicine. BMC Health Serv Res. 2010;10:220. doi: 10.1186/1472-6963-10-220. PubMed PMID: 20670418; PubMed Central PMCID: PMC2919531.

87. Nguyen HT, Grzywacz JG, Lang W, Walkup M, Arcury TA. Effects of Complementary Therapy on Health in a National U.S. Sample of Older Adults. Journal of Alternative \& Complementary Medicine. 2010;16(7):701-6. doi: 10.1089/acm.2009.0442.

88. Picciano MF, Dwyer JT, Radimer KL, Wilson DH, Fisher KD, Thomas PR, et al. Dietary supplement use among infants, children, and adolescents in the United States, 1999-2002. Arch Pediatr Adolesc Med. 2007;161(10):978-85. doi: 10.1001/archpedi.161.10.978. PubMed PMID: 17909142.

89. Blalock SJGPJPRANLLCLFJJM. Factors Associated with Potential Medication-Herb/natural Product Interactions in a Rural Community. Alternative Therapies in Health \& Medicine. 2009;15(5):26-34.

90. Birdee GS, Phillips RS, Davis RB, Gardiner P. Factors associated with pediatric use of complementary and alternative medicine. Pediatrics. 2010;125(2):249-56. doi: 10.1542/peds.2009-1406.

91. Barner JC, Bohman TM, Brown CM, Richards KM. Use of complementary and alternative medicine for treatment among African-Americans: A multivariate analysis. Research in Social and Administrative Pharmacy. 2010;6(3):196-208. doi: 10.1016/j.sapharm.2009.08.001.

92. Nahin RL, Dahlhamer JM, Taylor BL, Barnes PM, Stussman BJ, Simile CM, et al. Health behaviors and risk factors in those who use complementary and alternative medicine. BMC Public Health. 2007;7:217. doi: 10.1186/1471-2458-7-217. PubMed PMID: 17723149; PubMed Central PMCID: PMC2031902.

93. Chao MT, Wade C, Kronenberg F. Disclosure of complementary and alternative medicine to conventional medical providers: Variation by race/ethnicity and type of CAM. Journal of the National Medical Association. 2008;100(11):1341-9.

94. Tom Xu K, Farrell TW. The complementarity and substitution between unconventional and mainstream medicine among racial and ethnic groups in the United States. Health Serv Res. 2007;42(2):811-26. Epub 2007/03/17. doi: 10.1111/j.1475-6773.2006.00628.x. PubMed PMID: 17362219; PubMed Central PMCID: PMCPMC1955362.

95. Bardia A, Nisly NL, Zimmerman MB, Gryzlak BM, Wallace RB. Use of herbs among adults based on evidence-based indications: findings from the National Health Interview Survey. Mayo Clin Proc. 2007;82(5):561-6. Epub 2007/05/12. doi: 10.4065/82.5.561. PubMed PMID: 17493422; PubMed Central PMCID: PMCPMC1964882.

96. Upchurch DM, Dye CE, Chyu L, Gold EB, Greendale GA. Demographic, behavioral, and health correlates of complementary and alternative medicine and prayer use among midlife women: 2002. Journal of women's health. 2010;19(1):23-30. doi: 10.1089/jwh.2008.1096. PubMed PMID: 20088655; PubMed Central PMCID: PMC2828262.

97. Gardiner et al. Factors Associated with Herbal Therapy use by Adults in the United States. Alternative Therapies in Health \& Medicine. 2007;13(2):22-9.

98. Zhang Y, Fein EB, Fein SB. Feeding of dietary botanical supplements and teas to infants in the United States. Pediatrics. 2011;127(6):1060-6. doi: 10.1542/peds.2010-2294.

99. Srivastava KC, Mustafa T. Ginger (Zingiber officinale) in rheumatism and musculoskeletal disorders. Medical hypotheses.39(4):342-8. doi: 10.1016/0306-9877(92)90059-L. 
100. Srivastava KC. Aqueous extracts of onion, garlic and ginger inhibit platelet aggregation and alter arachidonic acid metabolism. Biomed Biochim Acta. 1984;43(8-9):S335-46. PubMed PMID: 6440548.

101. Mustafa T SK, Jensen KB. Drug development report 9. Pharmacology of ginger, Zingiber officinale. Journal of Drug Development. 1993;6:25-39.

102. Suekawa M, Yuasa K, Isono M, Sone H, Ikeya Y, Sakakibara I, et al. [Pharmacological studies on ginger. IV. Effect of (6)-shogaol on the arachidonic cascade]. Nihon yakurigaku zasshi Folia pharmacologica Japonica. 1986;88(4):263-9. Epub 1986/10/01. PubMed PMID: 3098654.

103. Sharma JN, Srivastava KC, Gan EK. Suppressive effects of eugenol and ginger oil on arthritic rats. Pharmacology. 1994;49(5):314-8. PubMed PMID: 7862743.

104. Shunro K, Morimitsu Y, Toshihiko O. Chemistry of Ginger Components and Inhibitory Factors of the Arachidonic Acid Cascade. Food Phytochemicals for Cancer Prevention II. ACS Symposium Series. 547: American Chemical Society; 1994. p. 244-50.

105. Ghazanfar SA. Handbook of Arabian medicinal plants. Boca Raton: CRC Press; 1994. 265 p. p.

106. World Health Organization. WHO monographs on selected medicinal plants. Geneva: World Health Organization; 1999-2009. v. <1-4> p.

107. Yasukawa K, Yamaguchi A, Arita J, Sakurai S, Ikeda A, Takido M. - Inhibitory effect of edible plant extracts on 12-O-tetradecanoylphorbol-13-acetate-induced ear oedema in mice. 1993;- 7(2):- 189.

108. Gobel H, Schmidt G, Soyka D. Effect of peppermint and eucalyptus oil preparations on neurophysiological and experimental algesimetric headache parameters. Cephalalgia : an international journal of headache. 1994;14(3):228-34; discussion 182. PubMed PMID: 7954745.

109. Gobel H, Schmidt G, Dworschak M, Stolze H, Heuss D. Essential plant oils and headache mechanisms. Phytomedicine. 1995;2(2):93-102. doi: 10.1016/S0944-7113(11)80053-X. PubMed PMID: 23196150.

110. Peana AT, Marzocco S, Popolo A, Pinto A. (-)-Linalool inhibits in vitro NO formation: Probable involvement in the antinociceptive activity of this monoterpene compound. Life sciences. 2006;78(7):719-23. doi: 10.1016/j.lfs.2005.05.065. PubMed PMID: 16137709.

111. Peana AT, Rubattu P, Piga GG, Fumagalli S, Boatto G, Pippia P, et al. Involvement of adenosine A1 and A2A receptors in (-)-linalool-induced antinociception. Life sciences. 2006;78(21):2471-4. doi: 10.1016/j.lfs.2005.10.025. PubMed PMID: 16343551.

112. Re L, Barocci S, Sonnino S, Mencarelli A, Vivani C, Paolucci G, et al. Linalool modifies the nicotinic receptor-ion channel kinetics at the mouse neuromuscular junction. Pharmacol Res. 2000;42(2):177-82. doi: 10.1006/phrs.2000.0671. PubMed PMID: 10887049.

113. Peana AT, D'Aquila PS, Panin F, Serra G, Pippia P, Moretti MD. Anti-inflammatory activity of linalool and linalyl acetate constituents of essential oils. Phytomedicine. 2002;9(8):721-6. doi: 10.1078/094471102321621322. PubMed PMID: 12587692.

114. Barnes PM, Powell-Griner E, McFann K, Nahin RL. Complementary and alternative medicine use among adults: United States 2002. Hyattsville, MD: National Center for Health Statistics, 2004 Contract No.: Report.

115. Arcury Q, Bell \& Vitolins. Complementary and alternativemedicine use among rural adults. Complementary Health Practice Review. 2002;7(3):167-86.

116. Bausell RB, Lee WL, Berman BM. Demographic and health-related correlates to visits to complementary and alternative medical providers. Medical care. 2001;39(2):190-6. PubMed PMID: 11176556.

117. Graham RE, Ahn AC, Davis RB, O'Connor BB, Eisenberg DM, Phillips RS. Use of complementary and alternative medical therapies among racial and ethnic minority adults: results from the 2002 National Health Interview Survey. J Natl Med Assoc. 2005;97(4):535-45. PubMed PMID: 15868773; PubMed Central PMCID: PMC2568705. 
118. Montalto CP, Bhargava, V., \& Hong, G. S. Use of complementary and alternative medicineby older adults: An exploratory study. Complementary Health Practice Review. 2006;11(1):27-46.

119. Tindle HA, Davis RB, Phillips RS, Eisenberg DM. Trends in use of complementary and alternative medicine by US adults: 1997-2002. Altern Ther Health Med. 2005;11(1):42-9. PubMed PMID: 15712765.

120. Bhargava V, Hong GS, Montalto CP. Use of practitioner-based and self-care complementary and alternative medicine in the united states: A demand for health perspective. Family and Consumer Sciences Research Journal. 2012;41(1):18-35. doi: 10.1111/j.1552-3934.2012.02126.x.

121. Grossman M. The demand for health: a theoretical and empirical investigation. New York,: National Bureau of Economic Research; distributed by Columbia University Press; 1972. xvii, 115 p. p.

122. Reisfield GM, Wasan AD, Jamison RN. The Prevalence and Significance of Cannabis Use in Patients Prescribed Chronic Opioid Therapy: A Review of the Extant Literature. Pain Medicine. 2009;10(8):1434-41.

\section{Chapter 5}

1. Gentile DA, Woodhouse J, Lynch P, Maier J, McJunkin T. Reliability and Validity of the Global Pain Scale with Chronic Pain Sufferers. Pain Physician. 2011;14:61-70.

2. Giacomelli C, Talarico R, Bombardieri S, Bazzichi L. The interaction between autoimmune diseases and fibromyalgia: risk, disease course and management. Expert review of clinical immunology. 2013;9(11):1069-76. doi: 10.1586/1744666X.2013.849440. PubMed PMID: 24168413.

3. Organization WH. WHO Monographs on Slected Medicinal Plants. 2003.

4. Carle R GK. Chamomile: a pharmacological and clinical profile. Drugs of Today. 1992;28:55965.

5. Gould L RC, Gomprecht RF. Cardiac effect of chamomile tea. Journal of clinical pharmacology. 1973;13:475-9.

6. Mascolo N, Autore G, Capasso F, Menghini A, Fasulo MP. - Biological screening of Italian medicinal plants for anti-inflammatory activity. 1987;- 1(- 1):- 31.

7. Tita B, Bello U, Faccendini P, et al. Taraxacum officinale W.: Pharmacological effect of an ethanol extract. Pharmacology Research. 1993;27(Suppl.1):23-4.

8. World Health Organization. WHO monographs on selected medicinal plants. Geneva: World Health Organization; 1999-2009. v. <1-4> p.

9. Yasukawa K, Yamaguchi A, Arita J, Sakurai S, Ikeda A, Takido M. - Inhibitory effect of edible plant extracts on 12-O-tetradecanoylphorbol-13-acetate-induced ear oedema in mice. 1993;- 7(2):- 189.

10. Delaveau P, Lallouette P, Tessier AM. [Stimulation of the phagocytic activity of R.E.S. by plant extracts (author's transl)]. Planta medica. 1980;40(1):49-54. doi: 10.1055/s-2008-1074941. PubMed PMID: 6999511.

11. Yesilada E, Ustun O, Sezik E, Takaishi Y, Ono Y, Honda G. Inhibitory effects of Turkish folk remedies on inflammatory cytokines: interleukin-1alpha, interleukin-1beta and tumor necrosis factor alpha. J Ethnopharmacol. 1997;58(1):59-73. PubMed PMID: 9324006.

12. al. WHe. In vitro inhibition of prostaglandin biosynthesis by essential oils and phenolic compounds. Plant Medica. 1986;3:184-7.

13. Yasukawa K, Takeuchi M, Takido M. Humulon, a bitter in the hop, inhibits tumor promotion by 12-O-tetradecanoylphorbol-13-acetate in two-stage carcinogenesis in mouse skin. Oncology. 1995;52(2):156-8. PubMed PMID: 7854777. 
14. H. H. Economic and medicinal plant research. Wagner H HH, Farnsworth NR, editor. London: Academic Press; 1985.

15. Duwiejua M, Zeitlin IJ, Waterman PG, Gray AI. Anti-inflammatory activity of Polygonum bistorta, Guaiacum officinale and Hamamelis virginiana in rats. The Journal of pharmacy and pharmacology. 1994;46(4):286-90. PubMed PMID: 8051612.

16. Cuellar MJ, Giner RM, Recio MC, Manez S, Rios JL. Topical anti-inflammatory activity of some Asian medicinal plants used in dermatological disorders. Fitoterapia. 2001;72(3):221-9. PubMed PMID: 11295297.

17. Butenko IG, Gladtchenko SV, Galushko SV. Anti-inflammatory properties and inhibition of leukotriene $\mathrm{C} 4$ biosynthesis in vitro by flavonoid baicalein from Scutellaria baicalensis georgy roots. Agents and actions. 1993;39 Spec No:C49-51. PubMed PMID: 8273583.

18. Kimura Y, Yokoi K, Matsushita N, Okuda H. Effects of flavonoids isolated from scutellariae radix on the production of tissue-type plasminogen activator and plasminogen activator inhibitor 1 induced by thrombin and thrombin receptor agonist peptide in cultured human umbilical vein endothelial cells. The Journal of pharmacy and pharmacology. 1997;49(8):816-22. Epub 1997/08/01. PubMed PMID: 9379363.

19. al. URe. American herbal pharmacopeia. Santa Cruz, CA: American Herbal Pharmacopeia; 1999.

20. Wagner I, Greim C, Laufer S, Heide L, Gleiter CH. Influence of willow bark extract on cyclooxygenase activity and on tumor necrosis factor alpha or interleukin 1 beta release in vitro and ex vivo. Clin Pharmacol Ther. 2003;73(3):272-4. Epub 2003/03/07. doi: 10.1067/mcp.2003.32. PubMed PMID: 12621392.

21. Chung CP, Park JB, Bae KH. Pharmacological effects of methanolic extract from the root of Scutellaria baicalensis and its flavonoids on human gingival fibroblast. Planta medica. 1995;61(2):150-3. Epub 1995/04/01. doi: 10.1055/s-2006-958036. PubMed PMID: 7753922.

22. Cheng GF, Liu DP, Yang DX, He KQ, Bai JY, Zhu XY. Antiinflammatory effects of Tremulacin, a Salicin-related substance isolated from Populus tomentosa Carr. leaves. Phytomedicine. 1994;1(3):209-11. doi: 10.1016/S0944-7113(11)80067-X. PubMed PMID: 23195941.

23. Srivastava KC, Mustafa T. Ginger (Zingiber officinale) in rheumatism and musculoskeletal disorders. Medical hypotheses.39(4):342-8. doi: 10.1016/0306-9877(92)90059-L.

24. Srivastava KC. Aqueous extracts of onion, garlic and ginger inhibit platelet aggregation and alter arachidonic acid metabolism. Biomed Biochim Acta. 1984;43(8-9):S335-46. PubMed PMID: 6440548.

25. Mustafa T SK, Jensen KB. Drug development report 9. Pharmacology of ginger, Zingiber officinale. Journal of Drug Development. 1993;6:25-39.

26. Suekawa M, Yuasa K, Isono M, Sone H, Ikeya Y, Sakakibara I, et al. [Pharmacological studies on ginger. IV. Effect of (6)-shogaol on the arachidonic cascade]. Nihon yakurigaku zasshi Folia pharmacologica Japonica. 1986;88(4):263-9. Epub 1986/10/01. PubMed PMID: 3098654.

27. Sharma JN, Srivastava KC, Gan EK. Suppressive effects of eugenol and ginger oil on arthritic rats. Pharmacology. 1994;49(5):314-8. PubMed PMID: 7862743.

28. Shunro K, Morimitsu Y, Toshihiko O. Chemistry of Ginger Components and Inhibitory Factors of the Arachidonic Acid Cascade. Food Phytochemicals for Cancer Prevention II. ACS Symposium Series. 547: American Chemical Society; 1994. p. 244-50.

29. Wang M, Guilbert LJ, Ling L, Li J, Wu Y, Xu S, et al. Immunomodulating activity of CVTE002, a proprietary extract from North American ginseng (Panax quinquefolium). The Journal of pharmacy and pharmacology. 2001;53(11):1515-23. PubMed PMID: 11732754.

30. Assinewe VA, Amason JT, Aubry A, Mullin J, Lemaire I. Extractable polysaccharides of Panax quinquefolius L. (North American ginseng) root stimulate TNFalpha production by alveolar macrophages. Phytomedicine. 2002;9(5):398-404. PubMed PMID: 12222658.

31. GB L. A pharmacometric evaluation of nine Bio-Strath herbal remedies. Medita. 1978;8:3-19. 
32. al CBe. Pharmacological properties of saponin fractions obtained from domestic crude drugs: Saponaria officinalis, Primula officinalis and Aesculus hippocastanum. Herba Polonica. 1976;22:154-62.

33. J B. Pharmacognosy, phytochemistry, medicinal plants. Paris: Lavoisier; 1995.

34. NG B. Max Wichtl's herbal drugs \& phytopharmaceuticals. Boca Raton, FL: CRC Press; 1994.

35. Bauer R WH. Echinacea species as potential immunostimulatory drugs. Economic and medicinal plants research. London: Academic Press; 1991. p. 253-321.

36. Wang H, Guo Y, Zhao X, Li H, Fan G, Mao H, et al. An estrogen receptor dependent mechanism of Oroxylin A in the repression of inflammatory response. PLoS One. 2013;8(7):e69555. doi:

10.1371/journal.pone.0069555. PubMed PMID: 23922737; PubMed Central PMCID: PMC3726624.

37. Audette JF, Bailey A. Integrative pain medicine : the science and practice of complementary and alternative medicine in pain management. Totowa, N.J.: Humana; 2008. xiv, 579 p. p.

38. NIH. Thunder God Vine 2012 [cited 2015 May 23]. Available from: https://nccih.nih.gov/health/tgvine.

39. Peana AT, D'Aquila PS, Panin F, Serra G, Pippia P, Moretti MD. Anti-inflammatory activity of linalool and linalyl acetate constituents of essential oils. Phytomedicine. 2002;9(8):721-6. doi: 10.1078/094471102321621322. PubMed PMID: 12587692.

40. O'Neil M, Hannah KL. Understanding the cultures of Prescription Drug Abuse, Misuse, Addiction, and Diversion. WV Medical Journal. 2010;106(4):64-70.

41. Ghazanfar SA. Handbook of Arabian medicinal plants. Boca Raton: CRC Press; 1994. 265 p. p.

42. Gobel H, Schmidt G, Soyka D. Effect of peppermint and eucalyptus oil preparations on neurophysiological and experimental algesimetric headache parameters. Cephalalgia : an international journal of headache. 1994;14(3):228-34; discussion 182. PubMed PMID: 7954745.

43. Gobel H, Schmidt G, Dworschak M, Stolze H, Heuss D. Essential plant oils and headache mechanisms. Phytomedicine. 1995;2(2):93-102. doi: 10.1016/S0944-7113(11)80053-X. PubMed PMID: 23196150. 
Tables

Chapter 2

Table 1: Characteristics of an Appalachian adult population by Fibromyalgia (FMS) status, Adjusted for Demographic and Lifestyle factors, C8 Health Project, 2005-2006

\begin{tabular}{|c|c|c|c|c|}
\hline \multirow[t]{2}{*}{ Characteristic } & Total Population & FMS & Non-FMS & \multirow{2}{*}{$\frac{\text { Adjusted Odds Ratio }^{\mathbf{a}}}{\mathrm{OR}(\mathrm{Cl}), p \text {-value }}$} \\
\hline & \multicolumn{3}{|c|}{$\mathrm{N}(\%) /$ Mean $(\mathrm{SD})$} & \\
\hline Total & 52535 & $1125(2.1 \%)$ & $51410(97.9 \%)$ & --- \\
\hline Age (years) & $45.25(16.14)$ & $51.14(11.58)$ & $45.12(16.20)$ & $1.02(1.02-1.03),<.0001^{*}$ \\
\hline \multicolumn{5}{|l|}{ Age } \\
\hline $18-24$ (ref) & $6540(12.5 \%)$ & $16 \quad(1.4 \%)$ & $6524(12.7 \%)$ & --- \\
\hline $25-44$ & $20178(38.4 \%)$ & $300(26.7 \%)$ & $19879(38.7 \%)$ & $3.89(2.30-6.56),<.0001^{*}$ \\
\hline $45-64$ & $19098(36.4 \%)$ & $689(61.2 \%)$ & $18409(35.8 \%)$ & $8.28(4.91-13.95),<.0001^{\star}$ \\
\hline $65+$ & $6719(12.8 \%)$ & $120(10.7 \%)$ & $6599(12.8 \%)$ & $4.37(2.50-7.64),<.0001^{*}$ \\
\hline \multicolumn{5}{|l|}{ Gender } \\
\hline Male (ref) & $25002(47.6 \%)$ & $90(8.0 \%)$ & $24912(48.5 \%)$ & --- \\
\hline Female & 27533 (52.4\%) & $1035(92.0 \%)$ & $26498(51.5 \%)$ & $10.68(8.53-13.37),<.0001^{*}$ \\
\hline \multicolumn{5}{|l|}{ Race/Ethnicity } \\
\hline Non-Hispanic White & $51092(97.2 \%)$ & $1098(97.6 \%)$ & $49994(97.2 \%)$ & 1.09 (0.73-1.62), .683 \\
\hline Other Race (ref) & $1443(2.8 \%)$ & $27(2.4 \%)$ & $1416(2.8 \%)$ & --- \\
\hline \multicolumn{5}{|l|}{ Education } \\
\hline$<12^{\text {th }}$ grade (ref) & $6261(11.9 \%)$ & $106(9.4 \%)$ & $6155(12.0 \%)$ & --- \\
\hline HS/GED & $22353(42.6 \%)$ & $445(39.6 \%)$ & $21908(42.6 \%)$ & $1.57(1.25-1.96), .0001^{*}$ \\
\hline Some College & $17081(32.5 \%)$ & $442(39.3 \%)$ & $16639(32.4 \%)$ & $2.22(1.75-2.82),<.0001^{\star}$ \\
\hline Bachelor's degree+ & $6840(13.0 \%)$ & $132(11.7 \%)$ & $6708(13.1 \%)$ & $1.91(1.42-2.56),<.0001^{*}$ \\
\hline \multicolumn{5}{|l|}{ Employment } \\
\hline Employed/Student(ref) & $33682(64.1 \%)$ & $482(42.8 \%)$ & $33200(64.6 \%)$ & --- \\
\hline Retired/Unemployed & $9057(17.2 \%)$ & $144(12.8 \%)$ & $8913(17.3 \%)$ & 1.44 (1.16-1.79), .0008* \\
\hline Disabled & 3357 (6.4\%) & $252(22.4)$ & $3105(6.0 \%)$ & $5.70(4.73-6.87),<.0001^{\star}$ \\
\hline Homemaker & $5979(11.4 \%)$ & $233(20.7 \%)$ & $5746(11.2 \%)$ & $1.50(1.26-1.78),<.0001^{*}$ \\
\hline Other & $460(0.88 \%)$ & $14(1.2 \%)$ & $446(0.9 \%)$ & $2.23(1.28-3.88), .004^{*}$ \\
\hline \multicolumn{5}{|l|}{ Marital Status } \\
\hline Married/Cohabitating (ref) & $36198(68.9 \%)$ & $836(74.3 \%)$ & $35362(68.8 \%)$ & --- \\
\hline Single & $8729(16.6 \%)$ & $60 \quad(5.3 \%)$ & $8669(16.9 \%)$ & $0.63(0.47-0.84), .002^{\star}$ \\
\hline Divorced/Sep/Widow & $7608(14.5 \%)$ & $229(20.4 \%)$ & $7379(14.4 \%)$ & $0.85(0.71-1.01), .058$ \\
\hline \multicolumn{5}{|l|}{ Household Income } \\
\hline Don't know/Missing & $5335 \quad(10.2 \%)$ & $92(8.2 \%)$ & $5243(10.2 \%)$ & 0.85 (0.66-1.10), .217 \\
\hline$<\$ 20,000$ (ref) & $12308(23.4 \%)$ & $288(25.6 \%)$ & $12020(23.4 \%)$ & --- \\
\hline$\$ 20,000-40,000$ & $14326(27.3 \%)$ & $301(26.8 \%)$ & $14025(27.3 \%)$ & $1.03(0.86-1.24), .759$ \\
\hline$\$ 40,001-70,000$ & $13559(25.8 \%)$ & $291(25.9 \%)$ & $13268(25.8 \%)$ & $1.05(0.86-1.29), .619$ \\
\hline$\$ 70,000+$ & 7007 (13.3\%) & $153(13.6 \%)$ & $6854(13.3 \%)$ & 1.09 (0.85-1.39), .514 \\
\hline \multicolumn{5}{|l|}{ Alcohol } \\
\hline Don't drink (ref) & 26847 (51.1\%) & $722(64.2 \%)$ & $26125(50.8 \%)$ & --- \\
\hline Currently Drink & $25688(48.9 \%)$ & $403(35.8 \%)$ & $25285(49.2 \%)$ & $0.86(0.75-0.98), .023^{*}$ \\
\hline \multicolumn{5}{|l|}{ Tobacco User } \\
\hline Never (ref) & $22859(43.5 \%)$ & $567(50.4 \%)$ & $22292(43.4 \%)$ & --- \\
\hline
\end{tabular}




\begin{tabular}{|c|c|c|c|c|}
\hline Current & $16691(31.8 \%)$ & $274(24.4 \%)$ & $16417(31.9 \%)$ & $0.91(0.78-1.07), .243$ \\
\hline Former & 12985 (24.7\%) & $284(25.2 \%)$ & $12701(24.7 \%)$ & $1.06(0.91-1.23), .441$ \\
\hline \multicolumn{5}{|l|}{ Exercise Program } \\
\hline No regularity (ref) & $36113(68.7 \%)$ & $762(67.7 \%)$ & $35351(68.8 \%)$ & --- \\
\hline Regular exercise & $16422(31.3 \%)$ & $363(32.3 \%)$ & $16059(31.2 \%)$ & --- \\
\hline Menopause occurred & $10526(38.2 \%)$ & $631(61.0 \%)$ & 9895 (37.3\%) & $1.82(1.49,2.21),<.0001^{*}$ \\
\hline
\end{tabular}

Note: Column Percentages shown a Model adjusted for demographic covariates Age, Gender, Marital Status, Employment, Education level,

White Race, and Household Income; Also adjusted for lifestyle covariates Tobacco Use and Current Alcohol consumption

Table 2: Health Characteristics of an Appalachian adult population by Fibromyalgia (FMS) status, Adjusted for Demographic and Lifestyle factors, C8 Health Project, 2005-2006

\begin{tabular}{|c|c|c|c|c|}
\hline Characteristic & $\begin{array}{c}\text { Total } \\
\text { Population }\end{array}$ & FMS & Non-FMS & Adjusted Odds Ratio ${ }^{a}$ \\
\hline & \multicolumn{3}{|c|}{$\mathrm{N}(\%) /$ Mean (SD) } & OR (Cl),p-value \\
\hline $\mathrm{BMI+}$ & $28.53(6.31)$ & $30.47(7.22)$ & $28.49(6.28)$ & $1.02(1.01-1.03), .001^{*}$ \\
\hline$\leq 24.99$ (ref) & $16258(31.0 \%)$ & $279(24.8 \%)$ & 15979 (31.1\%) & --- \\
\hline $25.5-29.99$ & $18200(34.6 \%)$ & $320(28.4 \%)$ & 17880 (34.8\%) & $1.11(.932-1.31), .248$ \\
\hline $30-34.9$ & $10704(20.4 \%)$ & $251(22.3 \%)$ & $10453(20.3 \%)$ & $1.24(1.02-1.49), .028^{*}$ \\
\hline $35+$ & $7373(14.0 \%)$ & 275 (24.4\%) & $7098(13.8 \%)$ & $1.43(1.18-1.74), .0003^{*}$ \\
\hline $\begin{array}{l}\text { Number of } \\
\text { Comorbidities }\end{array}$ & $1.29(1.27)$ & $2.78(1.59)$ & $1.25(1.24)$ & $1.60(1.54-1.67),<.0001^{*}$ \\
\hline \multicolumn{5}{|l|}{ Comorbidity Index } \\
\hline None (ref) & $16778(31.9 \%)$ & 58 (5.2\%) & $16720(32.5 \%)$ & --- \\
\hline 1 & 16999 (32.4\%) & $177(15.7 \%)$ & $16822(32.7 \%)$ & $2.38(1.77-3.21),<.0001^{*}$ \\
\hline 2 & $10535(20.1 \%)$ & $301(26.8 \%)$ & $10234(19.9 \%)$ & $5.16(3.88-6.86),<.0001^{*}$ \\
\hline $3+$ & $8223(15.7 \%)$ & $589(52.4 \%)$ & 7634 (14.9\%) & $10.46(7.90-13.84),<.0001^{*}$ \\
\hline Autoimmune disorder & $2192(4.2 \%)$ & $220(19.6 \%)$ & $1972(3.8 \%)$ & $3.43(2.89-4.06),<.0001^{*}$ \\
\hline Osteoarthritis & $4093(7.7 \%)$ & $411(35.5 \%)$ & $3782(7.1 \%)$ & $3.86(3.34-4.45),<.0001^{*}$ \\
\hline $\begin{array}{l}\text { Allergies or Chronic } \\
\text { Sinusitis }\end{array}$ & $24470(46.6 \%)$ & $893(79.4 \%)$ & 23577 (45.9\%) & $2.91(2.51-3.38),<.0001^{*}$ \\
\hline Kidney disorder & $5202 \quad(9.9 \%)$ & $226(20.1 \%)$ & $4976(9.7 \%)$ & $1.72(1.47-2.01),<.0001^{*}$ \\
\hline Respiratory disease & $7385(14.1 \%)$ & $342(30.4 \%)$ & $7043(13.7 \%)$ & $1.89(1.64-2.17),<.0001^{*}$ \\
\hline $\begin{array}{l}\text { Cardiovascular } \\
\text { disease }\end{array}$ & $4661 \quad(8.9 \%)$ & $148(13.2 \%)$ & $4513(8.9 \%)$ & $1.25(1.03-1.51), .027^{*}$ \\
\hline Liver disease & $684 \quad(1.3 \%)$ & 38 (3.4\%) & $646(1.3 \%)$ & $1.51(1.06-2.16), .022$ \\
\hline Endocrine Disorder & $4083(7.8 \%)$ & $264(23.5 \%)$ & $3819(7.4 \%)$ & $1.82(1.57-2.12),<.0001^{*}$ \\
\hline Diabetes & $4778(9.1 \%)$ & $157(14.0 \%)$ & $4621(9.0 \%)$ & $.978(.813-1.18), .810$ \\
\hline Frequent Headache & $10105(19.2 \%)$ & $439(39.0 \%)$ & $9666(18.8 \%)$ & $2.03(1.78-2.31),<.0001^{*}$ \\
\hline $\begin{array}{l}\text { Sleep Impairment } \\
\text { Scores }^{\text {s }}\end{array}$ & $4.00(3.56)$ & $7.32(3.29)$ & $3.93(3.53)$ & $1.23(1.21-1.25),<.0001^{*}$ \\
\hline $\begin{array}{l}\text { Mood Disturbance } \\
\text { Score }^{m}\end{array}$ & $4.59(3.70)$ & $7.25(3.40)$ & $4.53(3.68)$ & $1.19(1.17-1.21),<.0001^{*}$ \\
\hline
\end{tabular}


Table 4: Model Statistics assessing the association between Fibromyalgia (FMS) and blood serum C-reactive Protein (CRP) quartile adjusting for Sleep impairment and Mood disturbance in an Appalachian adult population, 2005-2006 (N=52,535)

\begin{tabular}{|c|c|c|c|c|c|c|}
\hline & \multicolumn{5}{|c|}{ 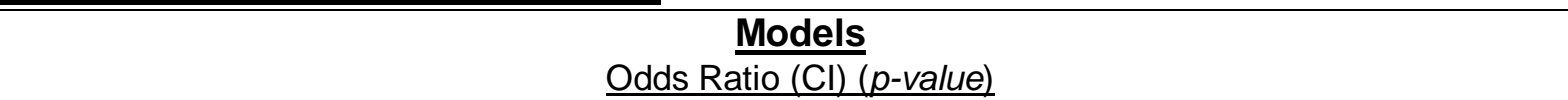 } \\
\hline & & $\underline{\text { Crude }}^{\mathrm{e}}$ & $\begin{array}{l}\frac{\text { Adjusted for }}{\text { Demographic and }} \\
\frac{\text { Lifestyle Factors }}{\text { (Model 1) }^{f}}\end{array}$ & 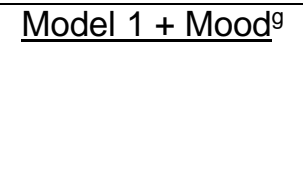 & ${\text { Model } 1+\text { Sleep }^{h}}^{h}$ & $\begin{array}{l}\text { Model 1+ } \\
\text { Mood \& Sleep }^{r}\end{array}$ \\
\hline \multicolumn{2}{|c|}{ CRP level (mg/L) } & $\begin{array}{l}1.02(1.01-1.02) \\
\left(<.0001^{*}\right)\end{array}$ & $1.01(1.00-1.01)\left(.004^{*}\right)$ & $1.01(1.00-1.01)\left(.021^{*}\right)$ & $1.01(1.00-1.01)\left(.035^{\star}\right)$ & $\begin{array}{l}1.01(1.00-1.01) \\
\left(.031^{*}\right)\end{array}$ \\
\hline \multirow{4}{*}{$\frac{\mathrm{CRP}}{\text { (ref) }}$} & Quartile $1^{a}$ & -- & --- & --- & --- & -- \\
\hline & Quartile $2^{b}$ & $1.33(1.09-1.62)\left(.004^{*}\right)$ & $1.13(0.92-1.38)(.243)$ & $1.12(0.91-1.36)(.287)$ & $1.10(0.90-1.35)(.357)$ & $\begin{array}{l}1.10(0.90-1.34) \\
(.363)\end{array}$ \\
\hline & Quartile $3^{c}$ & $1.78(1.48-2.14)\left(<.0001^{*}\right)$ & $1.23(1.02-1.48)\left(.032^{*}\right)$ & $1.20(1.00-1.45)(.056)$ & $1.18(0.98-1.43)(.083)$ & $\begin{array}{l}1.18(0.97-1.42) \\
(.093)\end{array}$ \\
\hline & Quartile $4^{d}$ & $2.50(2.10-2.97)\left(<.0001^{*}\right)$ & $1.35(1.13-1.62)\left(.0009^{*}\right)$ & $1.28(1.07-1.53)\left(.007^{*}\right)$ & $1.26(1.05-1.51)\left(.011^{*}\right)$ & $\begin{array}{l}1.24(1.03-1.48) \\
\left(.021^{*}\right)\end{array}$ \\
\hline \multicolumn{2}{|c|}{ Wald $X^{2}, p$ for trend ${ }^{t}$} & $118.42,\left(<.0001^{*}\right)$ & $17.48,\left(<.0001^{*}\right)$ & $11.06,\left(.0009^{\star}\right)$ & $10.40,\left(.001^{\star}\right)$ & $8.25,\left(.004^{\star}\right)$ \\
\hline
\end{tabular}

a C-reactive protein $.15-.80 \mathrm{mg} / \mathrm{Lb}^{\mathrm{b}}$

C-reactive protein $.81-1.80 \mathrm{mg} / \mathrm{L}$

C-reactive protein $1.81-4.20 \mathrm{mg} / \mathrm{L}$

${ }^{d}$ C-reactive protein $4.21-250.6 \mathrm{mg} / \mathrm{L}$

t Linear trend ${ }^{\mathrm{e}}\left(\mathrm{R}^{2=}=.003\right)^{\text {f }}$ Full Model adjusted for demographic covariates Age, Gender, Marital Status, Employment, Education level, White Race, and Household Income; Also adjusted for lifestyle covariates Tobacco Use and Current Alcohol consumption $\left(R^{2}=.031\right)$

9 Mood disturbance derived from responses to four individual questions regarding frequent mood swings, irritability, fatigue and/or inability to concentrate $\left(R^{2}=.035\right)$

${ }^{\mathrm{h}}$ Sleep impairment derived from responses to four individual questions regarding the frequency of short sleep, fitful sleep, insomnia and/or daytime somnolence

$\left(\mathrm{R}^{2}=.037\right)$

$r\left(R^{2}=.038\right)$

\section{Chapter 3}


Table 1a: Demographic and Lifestyle Characteristics of two National U.S. samples (NHIS 2007 \& 2012), stratified by Herbal use (previous or current use of herbal products)

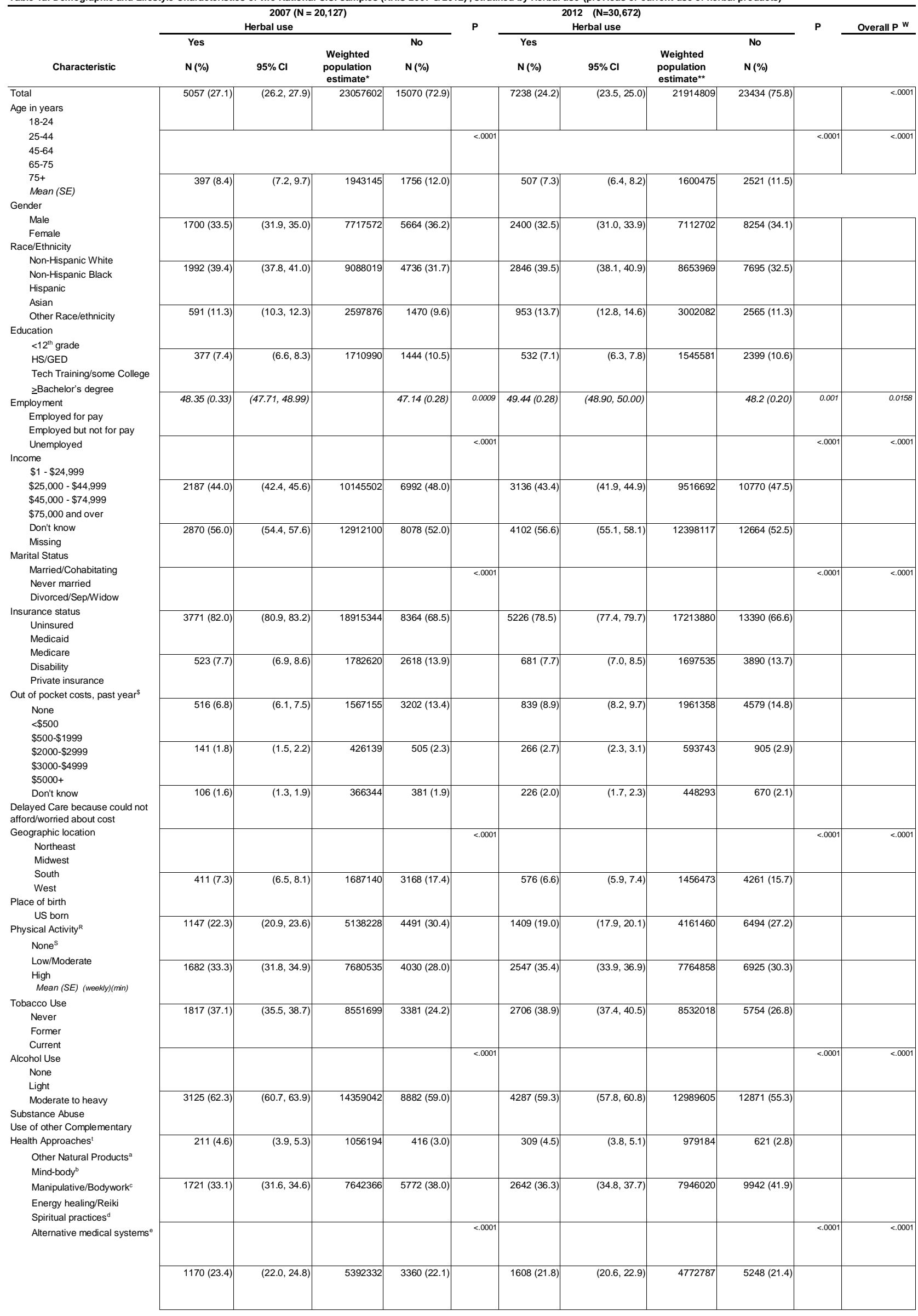




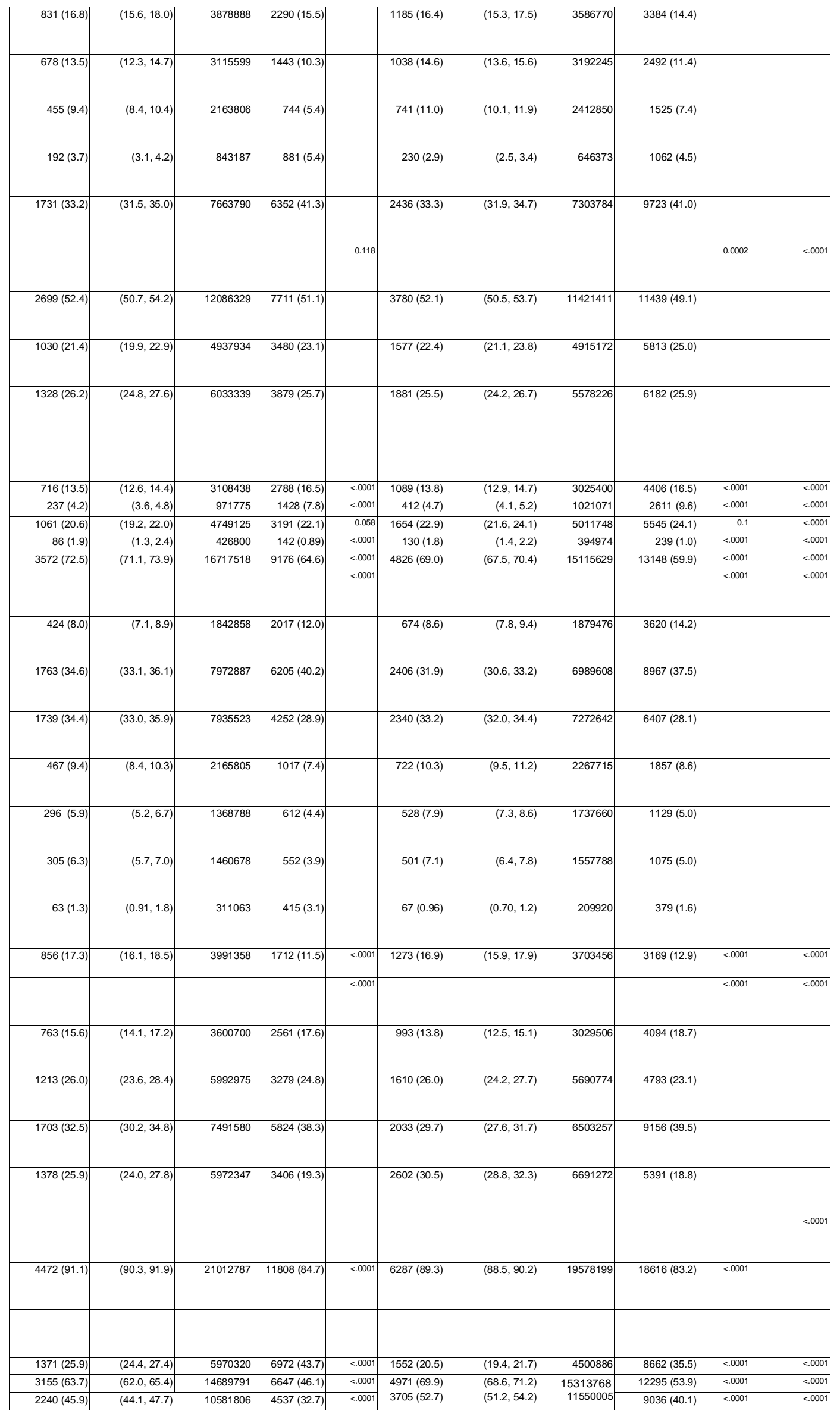




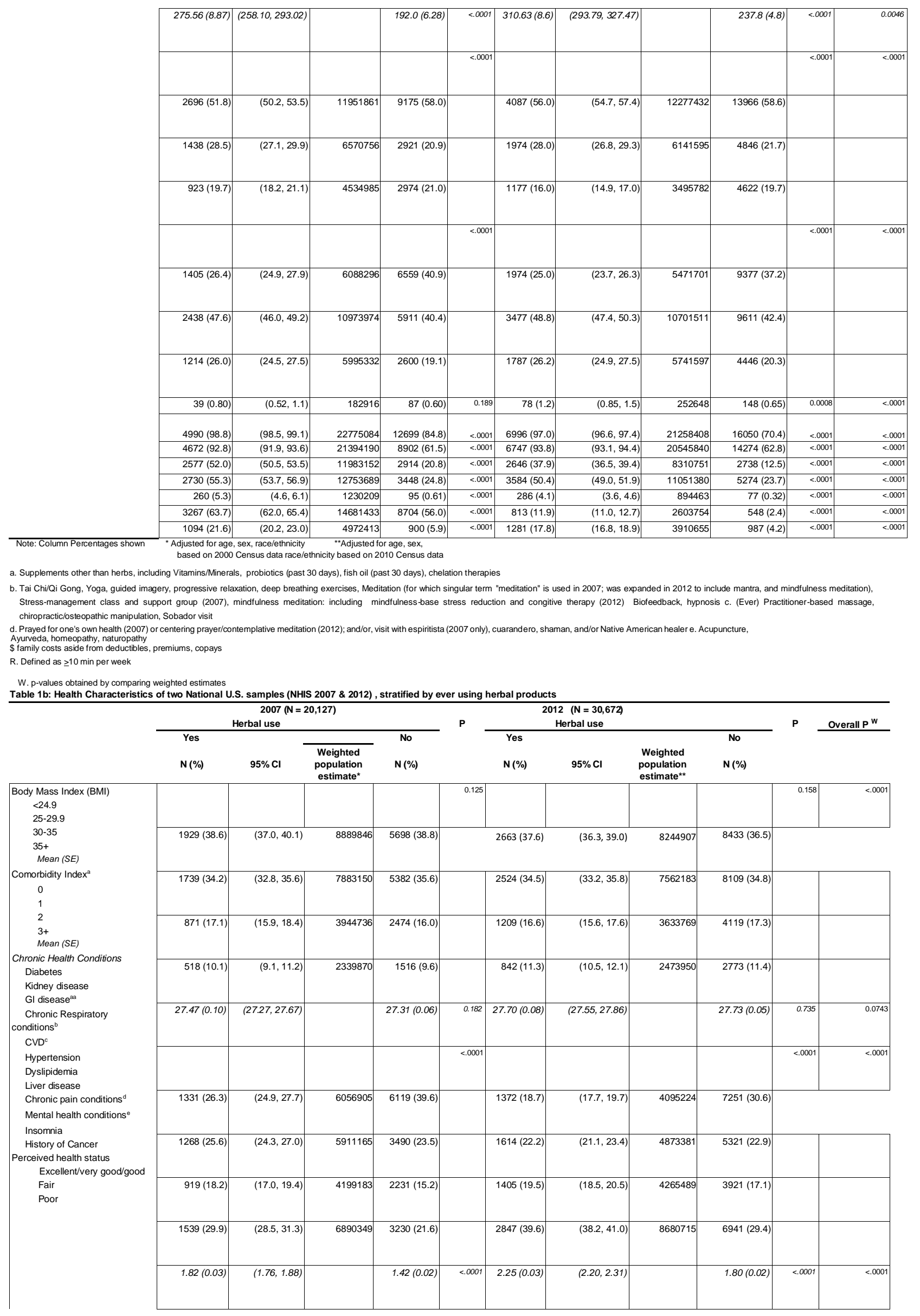




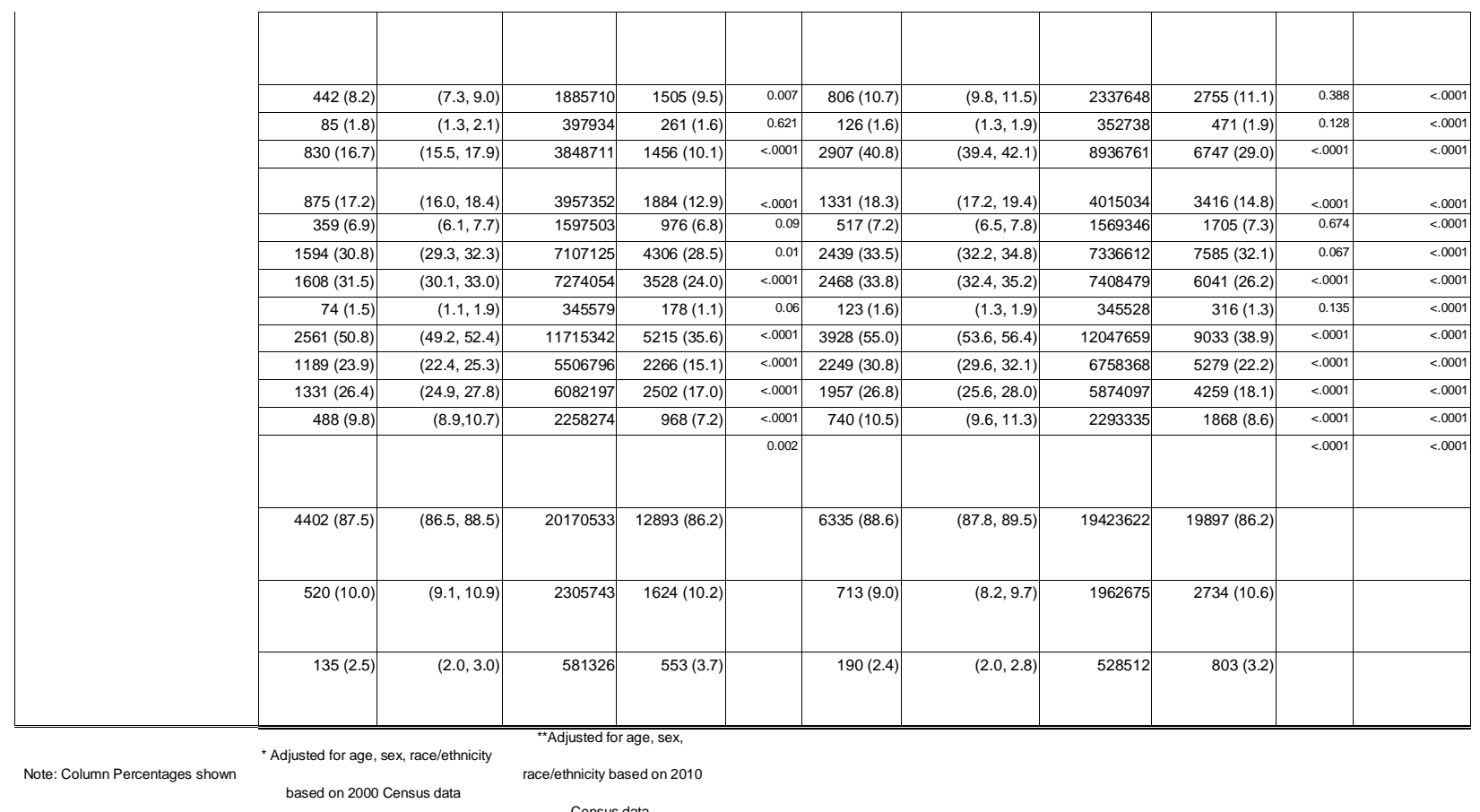

a. Includes diabetes, kidney disease, Gastrointestinal disorder (including ulcer and/or abdominal pain, digestive allergy, heartburn), respiratory conditions (Asthma, emphesema, and.or chronic bronchitis), dyslipidemia, liver condition, Rheumatoid Arthritis, CVD, hypertension, non-specific arthritis, gout, migraines, mental health condition

aa. Includes: bowel conditions and ulcers (2007); past 12 month abdominal pain, digestive allergy, heartburn, and ever having ulcer (2012) b. Asthma, emphysema, and/or chronic bronchitis

c. Myocardial Infarction, Heart Disease, Angina pectoris

d. Migraines (past 3 months), nonspecific arthritis, gout, non-specific joint pain for $3+$ months, Rheumatoid Arthritis

e. Depression, bipolar disorder, phobias, often anxious in past year

W. p-values obtained by comparing weighted estimates

Table 2a: Demographic and Lifestyle Characteristics of two National U.S. samples (NHIS 2007 \& 2012), stratified by herbal use in the last 12 months

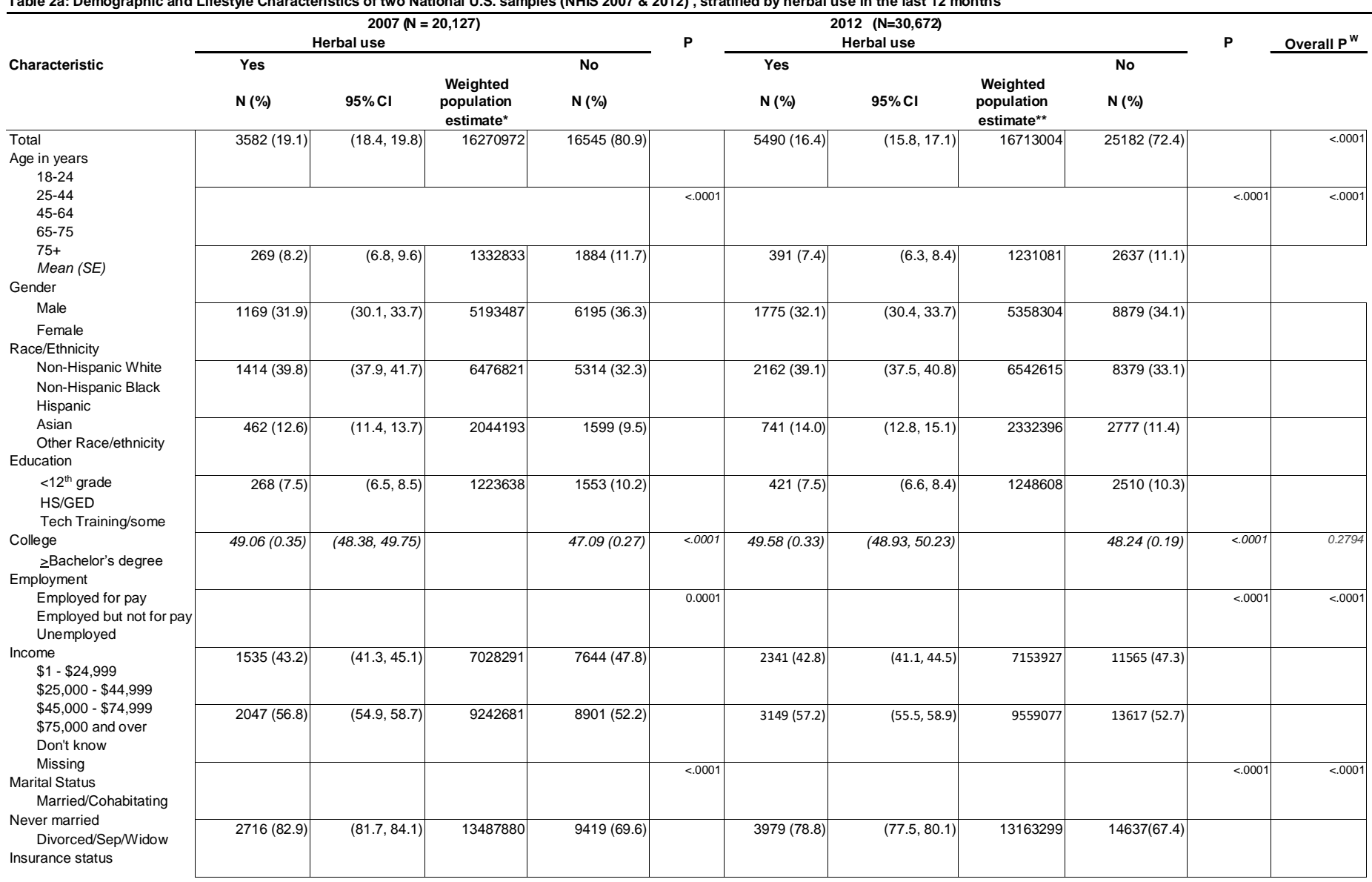




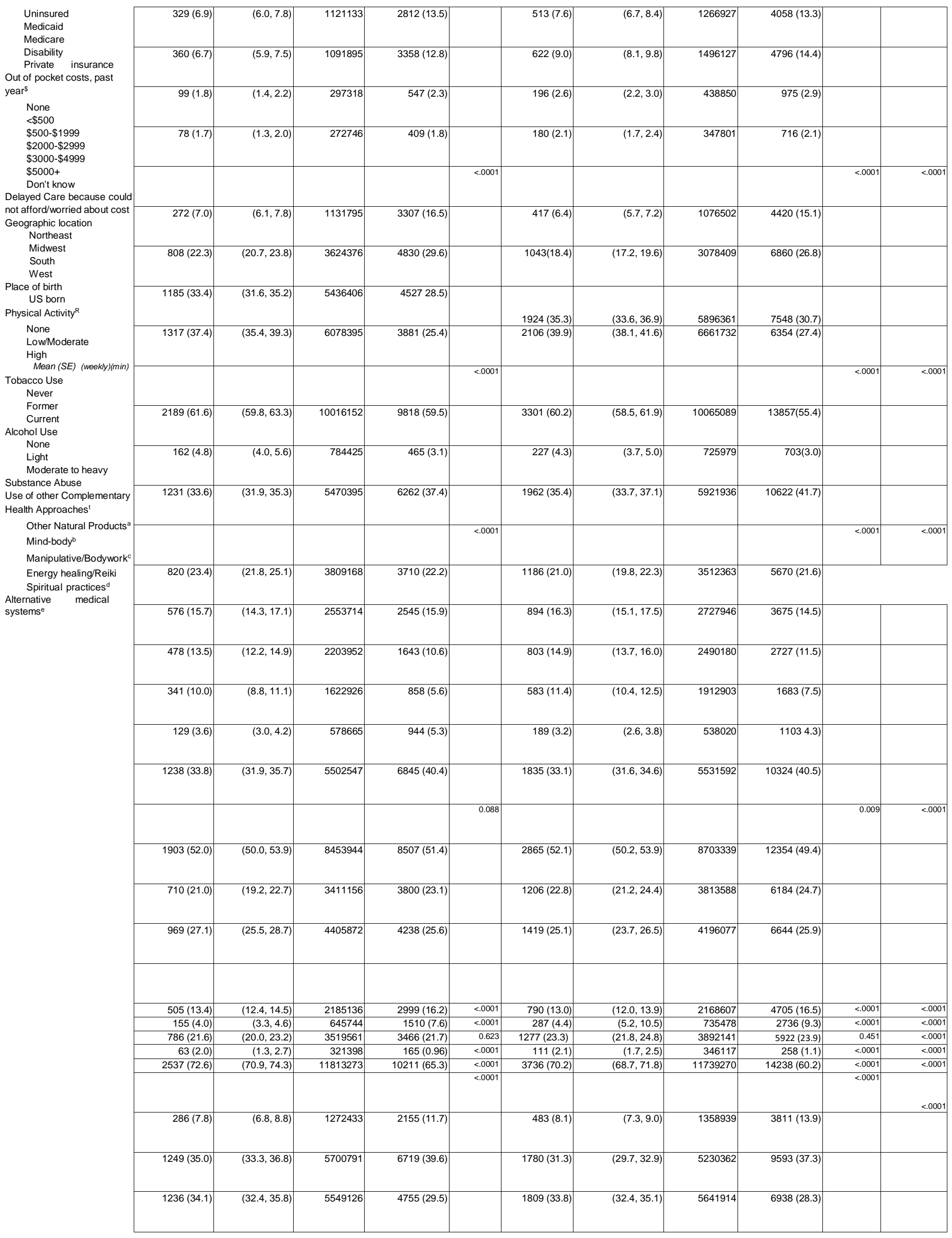




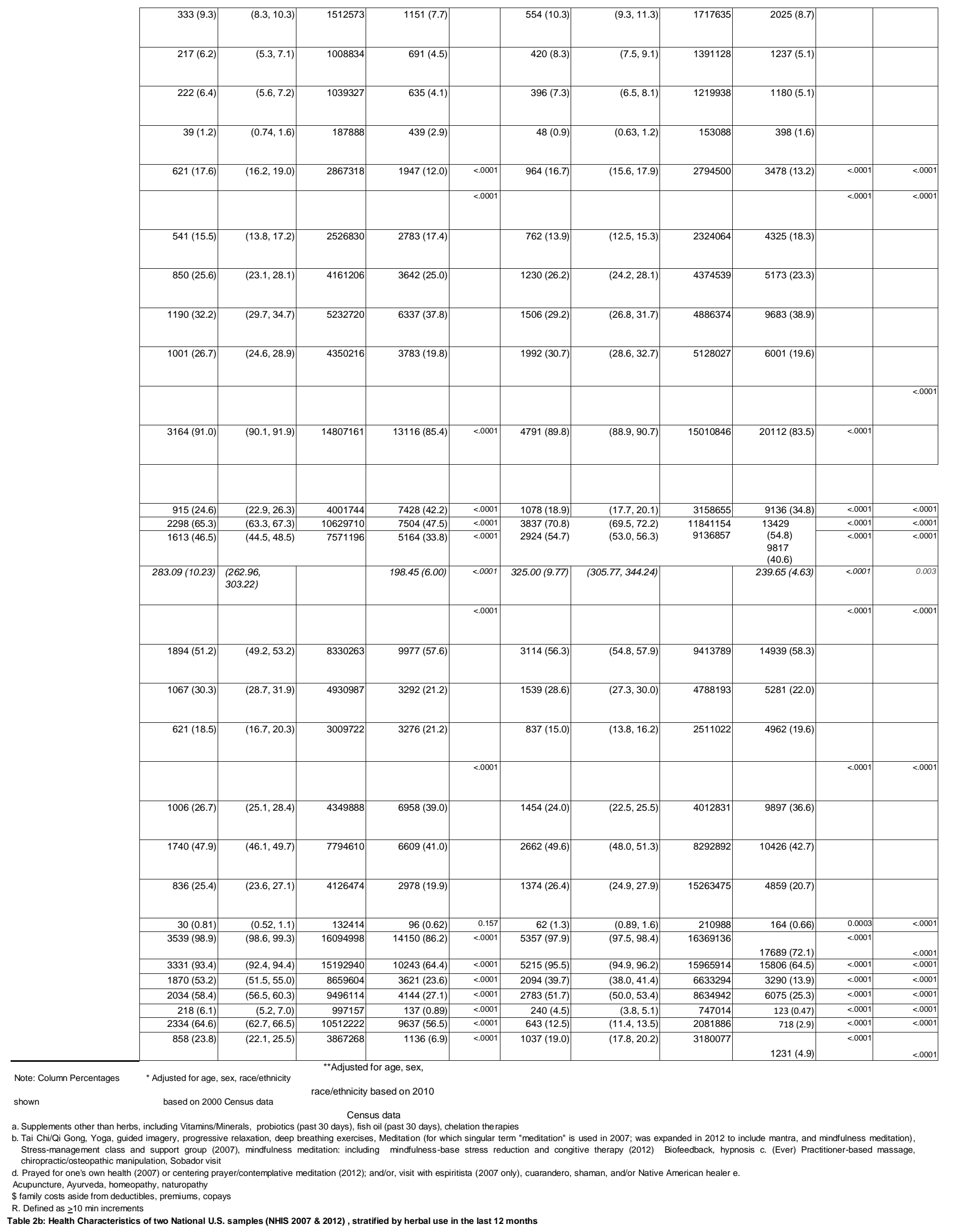




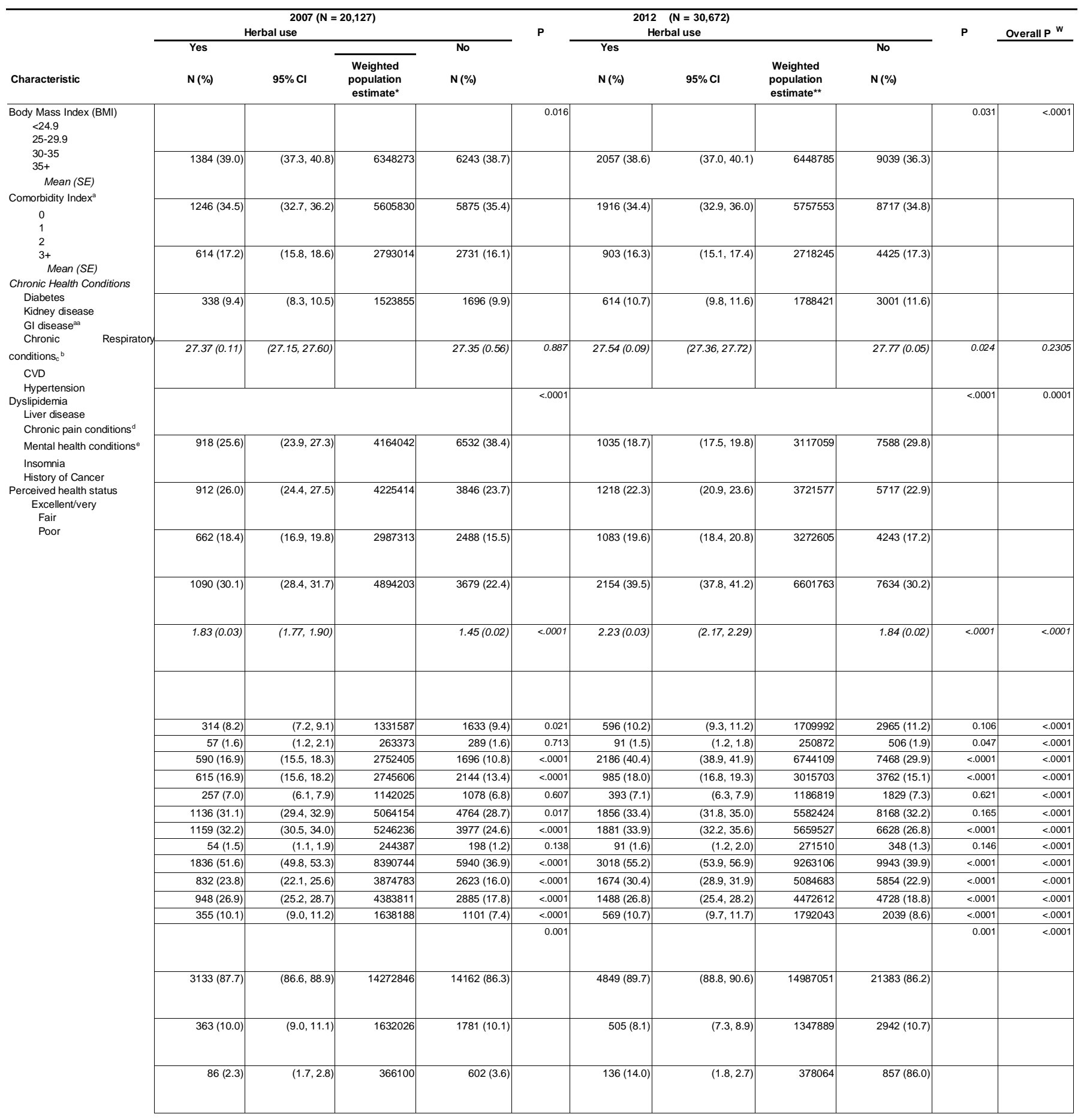

${ }^{*}$ Adjusted for age, sex, race/ethnicity ${ }^{* *}$ Adjusted for age, sex, race/ethnicily
Note: Column Percentages show based on 2000 Census data

a. Includes diabetes, kidney disease, Gastrointestinal disorder (including ulcer and/or abdominal pain, digestive allergy, heartburn), respiratory conditions (Asthma, emphesema, and.or chronic bronchitis), dyslipidemia, liver condition, Rheumatoid Arthritis, CVD, hypertension, non-specific arthritis, gout, migraines, mental health condition

aa. Includes: bowel conditions and ulcers (2007); past 12 month abdominal pain, digestive allergy, heartburn, and ever having ulcer (2012)

b. Asthma, emphysema, and/or chronic bronchitis

c. Myocardial Infarction, Heart Disease, Angina pectoris

d. Migraines (past 3 months), nonspecific arthritis, gout, non-specific joint pain for $3+$ months, Rheumatoid Arthritis

e. Depression, bipolar disorder, phobias, often anxious in past year

W. p-values obtained by comparing weighted estimates 
Table 3a: Demographic and Lifestyle Characteristics of two National U.S. samples (NHIS 2007 \& 2012), stratified by herbal use in past 30 days

\begin{tabular}{|c|c|c|c|c|c|c|c|c|c|c|c|}
\hline \multirow{4}{*}{ Characteristic } & \multirow{2}{*}{\multicolumn{4}{|c|}{$\begin{array}{l}\qquad 2007(\mathrm{~N}=20,127) \\
\text { Herbal use }\end{array}$}} & \multirow{2}{*}{\multicolumn{5}{|c|}{$\begin{array}{c}2012(\mathrm{~N}=30,672) \\
\text { Herbal use }\end{array}$}} & \multirow{4}{*}{$\mathbf{P}$} & \multirow{4}{*}{ Overall $\mathrm{P}^{\mathrm{W}}$} \\
\hline & & & & & & & & & & & \\
\hline & \multicolumn{2}{|l|}{ Yes } & \multirow[b]{2}{*}{$\begin{array}{l}\text { Weighted } \\
\text { population } \\
\text { estimate }^{\star}\end{array}$} & No & & Yes & & & No & & \\
\hline & N (\%) & $95 \% \mathrm{Cl}$ & & $\mathbf{N}(\%)$ & & $\mathrm{N}(\%)$ & $95 \% \mathrm{Cl}$ & $\begin{array}{l}\text { Weighted } \\
\text { population } \\
\text { estimate }^{\star \star}\end{array}$ & $\mathrm{N}(\%)$ & & \\
\hline Total & $2623(13.9)$ & $(13.3,14.6)$ & 11859568 & $17504(86.1)$ & & 4120 (13.9) & $(13.4,14.5)$ & 12603256 & $26552(86.1)$ & & $<.0001$ \\
\hline $\begin{array}{c}\text { Age in years } \\
18-24\end{array}$ & & & & & & & & & & & \\
\hline $\begin{array}{l}25-44 \\
45-64 \\
65-75\end{array}$ & & & & & $<.0001$ & & & & & $<<.0001$ & $<.0001$ \\
\hline $\begin{array}{l}\text { 75+ } \\
\text { Mean (SE) } \\
\text { Gender }\end{array}$ & $155(6.7)$ & \begin{tabular}{|c|}
$(5.2,8.1)$ \\
\end{tabular} & 790687 & 1998 (11.7) & & $208(5.2)$ & $\begin{array}{l}(4.2,6.2) \\
\end{array}$ & 658449 & $2820(11.3)$ & & \\
\hline $\begin{array}{l}\text { Male } \\
\text { Female } \\
\text { Race/Ethnicity }\end{array}$ & 754 (28.2) & $(26.1,30.2)$ & 3340364 & $6610(36.6)$ & & $1165(28.0)$ & $(26.2,29.8)$ & 3529186 & 9489 (34.6) & & \\
\hline $\begin{array}{l}\text { Non-Hispanic White } \\
\text { Non-Hispanic Black } \\
\text { Hispanic }\end{array}$ & $1100(42.3)$ & $(40.1,44.5)$ & 5014017 & $5628(32.4)$ & & 1717 (41.3) & $(39.5,43.1)$ & 5207028 & $8824(33.1)$ & & \\
\hline $\begin{array}{l}\text { Asian } \\
\text { Other Race/ethnicity } \\
\text { Education }\end{array}$ & $392(14.3)$ & $(12.9,15.8)$ & 1697704 & $1669(9.4)$ & & $661(16.8)$ & $(15.4,18.1)$ & 2111431 & $2857(11.1)$ & & \\
\hline $\begin{array}{l}<12^{\text {th }} \text { grade } \\
\text { HS/GED } \\
\text { Tech Training/some College }\end{array}$ & $222(8.6)$ & $\begin{array}{l}(7.4,9.8) \\
\end{array}$ & 1016796 & 1599 (9.9) & & $369(8.7)$ & $(7.6,9.8)$ & 1097162 & $2562(9.9)$ & & \\
\hline $\begin{array}{l}\geq \text { Bachelor's degree } \\
\text { Employment } \\
\text { Employed for pay }\end{array}$ & $51.05(0.39)$ & $(50.29,51.82)$ & & $46.90(0.26)$ & $<.0001$ & $52.06(0.36)$ & $(51.35,52.76)$ & & $47.91(0.19)$ & $<.0001$ & 0.0592 \\
\hline $\begin{array}{l}\text { Employed but not for pay } \\
\text { Unemployed } \\
\text { Income }\end{array}$ & & & & & 0.022 & & & & & $<.0001$ & $<.0001$ \\
\hline $\begin{array}{l}\$ 1-\$ 24,999 \\
\$ 25,000-\$ 44,999 \\
\$ 45,000-\$ 74,999\end{array}$ & $1154(44.6)$ & $(42.5,46.8)$ & 5291066 & 8025 (47.3) & & 1731 (42.1) & $(40.2,44.1)$ & 5311074 & $12175(47.2)$ & & \\
\hline $\begin{array}{l}\$ 75,000 \text { and over } \\
\text { Don't know } \\
\text { Missing }\end{array}$ & $1469(55.4)$ & $(53.2,57.5)$ & 6568502 & 9479 (52.7) & & 2389 (57.9) & $(55.9,59.8)$ & 7292182 & $14377(52.8)$ & & \\
\hline $\begin{array}{l}\text { Marital Status } \\
\text { Married/Cohabitating } \\
\text { Never married }\end{array}$ & & & & & $<.0001$ & & & & & $<.0001$ & $<.0001$ \\
\hline $\begin{array}{l}\text { Divorced/Sep/Widow } \\
\text { Insurance status } \\
\text { Uninsured }\end{array}$ & $2020(83.8)$ & $(82.4,85.3)$ & 9939278 & $10115(70.3)$ & & 3065 (80.4) & $(79.1,81.8)$ & 10135774 & $15551(67.7)$ & & \\
\hline $\begin{array}{l}\text { Medicaid } \\
\text { Medicare } \\
\text { Disability }\end{array}$ & $218(6.1)$ & $(5.1,7.1)$ & 725090 & $2923(13.2)$ & & $351(6.9)$ & $(6.0,7.8)$ & 871677 & $4220(13.1)$ & & \\
\hline $\begin{array}{l}\text { Private insurance } \\
\text { Out of pocket costs, past year } \\
\text { None }\end{array}$ & $258(6.6)$ & $(5.7,7.5)$ & 787286 & $3460(12.4)$ & & $419(8.1)$ & $(7.3,8.9)$ & 1017455 & $4999(14.2)$ & & \\
\hline $\begin{array}{l}<\$ 500 \\
\$ 500-\$ 1999 \\
\$ 2000-\$ 2999\end{array}$ & $76(1.9)$ & $(1.4,2.5)$ & 229743 & $570(2.3)$ & & $139(2.4)$ & $(1.9,2.8)$ & 302167 & $1032(2.9)$ & & \\
\hline $\begin{array}{l}\$ 3000-\$ 4999 \\
\$ 5000+ \\
\text { Don't know }\end{array}$ & $51(1.5)$ & $(1.1,1.9)$ & 178171 & $436(1.8)$ & & $146(2.2)$ & $(1.7,2.7)$ & 276183 & $750(2.0)$ & & \\
\hline $\begin{array}{l}\text { Delayed Care because could not } \\
\text { afford/worried about cost } \\
\text { Geographic location }\end{array}$ & & & & & $<.0001$ & & & & & $<.0001$ & $<.0001$ \\
\hline $\begin{array}{l}\text { Midwest } \\
\text { South } \\
\text { West }\end{array}$ & $193(6.6)$ & $(5.7,7.5)$ & 783942 & 3386 (16.0) & & $315(6.5)$ & $(5.6,7.4)$ & 814077 & $4522(14.7)$ & & \\
\hline $\begin{array}{l}\text { Place of birth } \\
\text { US born } \\
\text { Physical Activity }\end{array}$ & $602(22.8)$ & $(21.1,24.6)$ & 2706476 & $5036(29.1)$ & & $801(18.7)$ & $(17.3,20.2)$ & 2362123 & $7102(26.3)$ & & \\
\hline $\begin{array}{l}\text { None } \\
\text { Low/Moderate }\end{array}$ & $837(31.9)$ & $(29.8,34.0)$ & 3780874 & $4875(29.0)$ & & $1403(34.5)$ & $(32.6,36.4)$ & 4348552 & $8069(31.1)$ & & \\
\hline $\begin{array}{l}\text { Mean (SE) (weekly)(min) } \\
\text { Tobacco Use } \\
\text { Never }\end{array}$ & 991 (38.7) & $(36.4,41.0)$ & 4588276 & 4207 (25.9) & & 1601 (40.3) & $(38.4,42.2)$ & 5078504 & $6859(28.0)$ & & \\
\hline $\begin{array}{l}\text { Former } \\
\text { Current }\end{array}$ & & & & & 0.002 & & & & & 0.0005 & $<.0001$ \\
\hline $\begin{array}{l}\text { Alcohol Use } \\
\text { None } \\
\quad \text { Light } \\
\quad \text { Moderate to heavy }\end{array}$ & $1546(59.7)$ & $(57.6,61.8)$ & 7078796 & $10461(60.0)$ & & 2384 (57.9) & $(56.0,59.7)$ & 7291127 & $14774(56.0)$ & & \\
\hline $\begin{array}{l}\text { Substance Abuse } \\
\text { Use of other Complementary Healtt } \\
\text { Approache }\end{array}$ & $121(4.7)$ & $(3.8,5.6)$ & 553445 & $506(3.2)$ & & $168(4.1)$ & $(3.4,4.9)$ & 521755 & $762(3.1)$ & & \\
\hline $\begin{array}{l}\text { Other Natural Products }{ }^{\mathrm{a}} \\
\text { Mind-body }{ }^{\mathrm{b}}\end{array}$ & 956 (35.7) & $(33.6,37.7)$ & 4227327 & $6537(36.8)$ & & 1568 (38.0) & $(36.1,39.9)$ & 4790374 & $11016(40.9)$ & & \\
\hline $\begin{array}{l}\text { Manipulative/Bodywork } \\
\text { Energy healing/Reiki } \\
\text { Spiritual practices }\end{array}$ & & & & & $<.0001$ & & & & & $<.0001$ & $<.0001$ \\
\hline
\end{tabular}




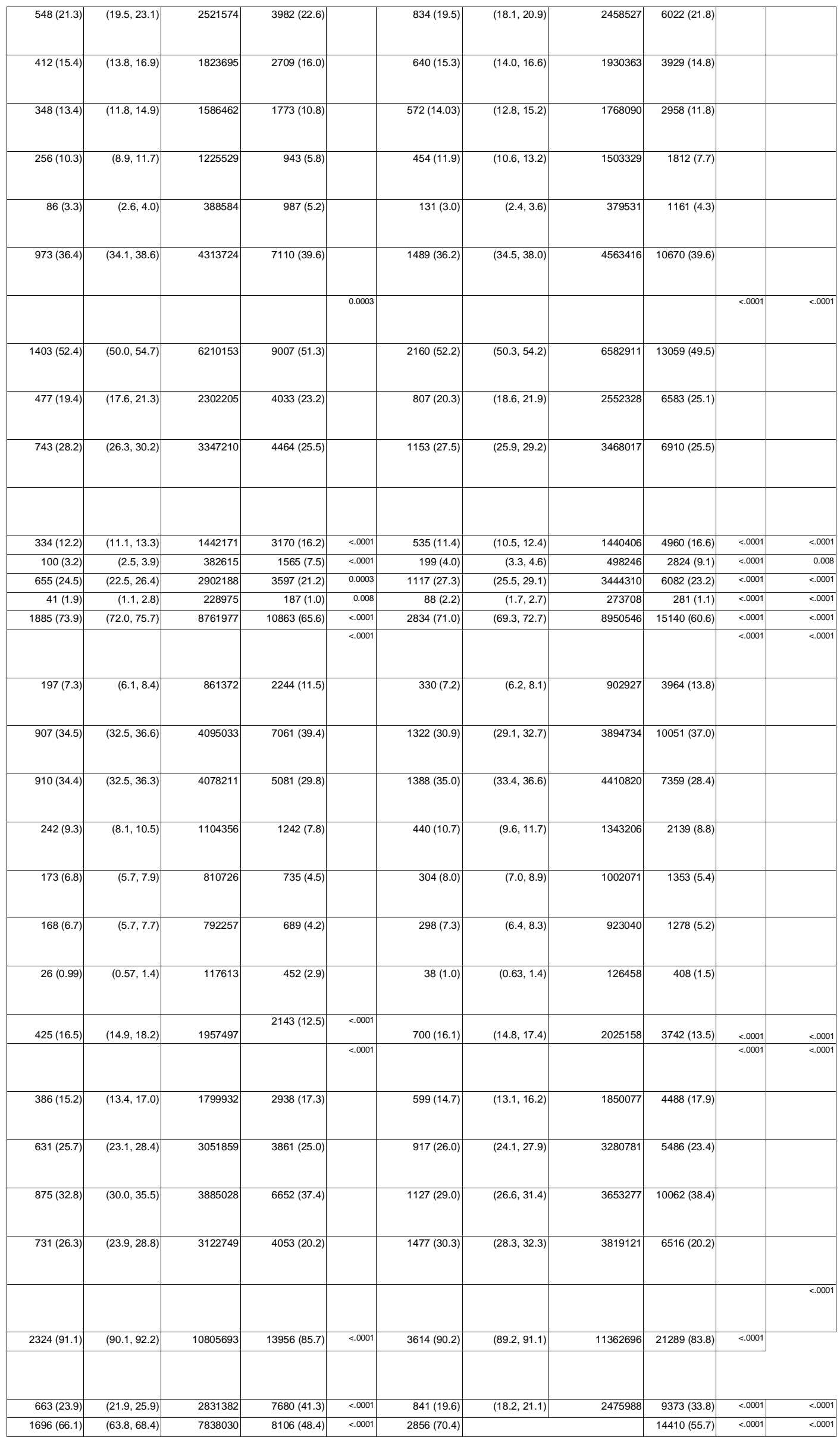




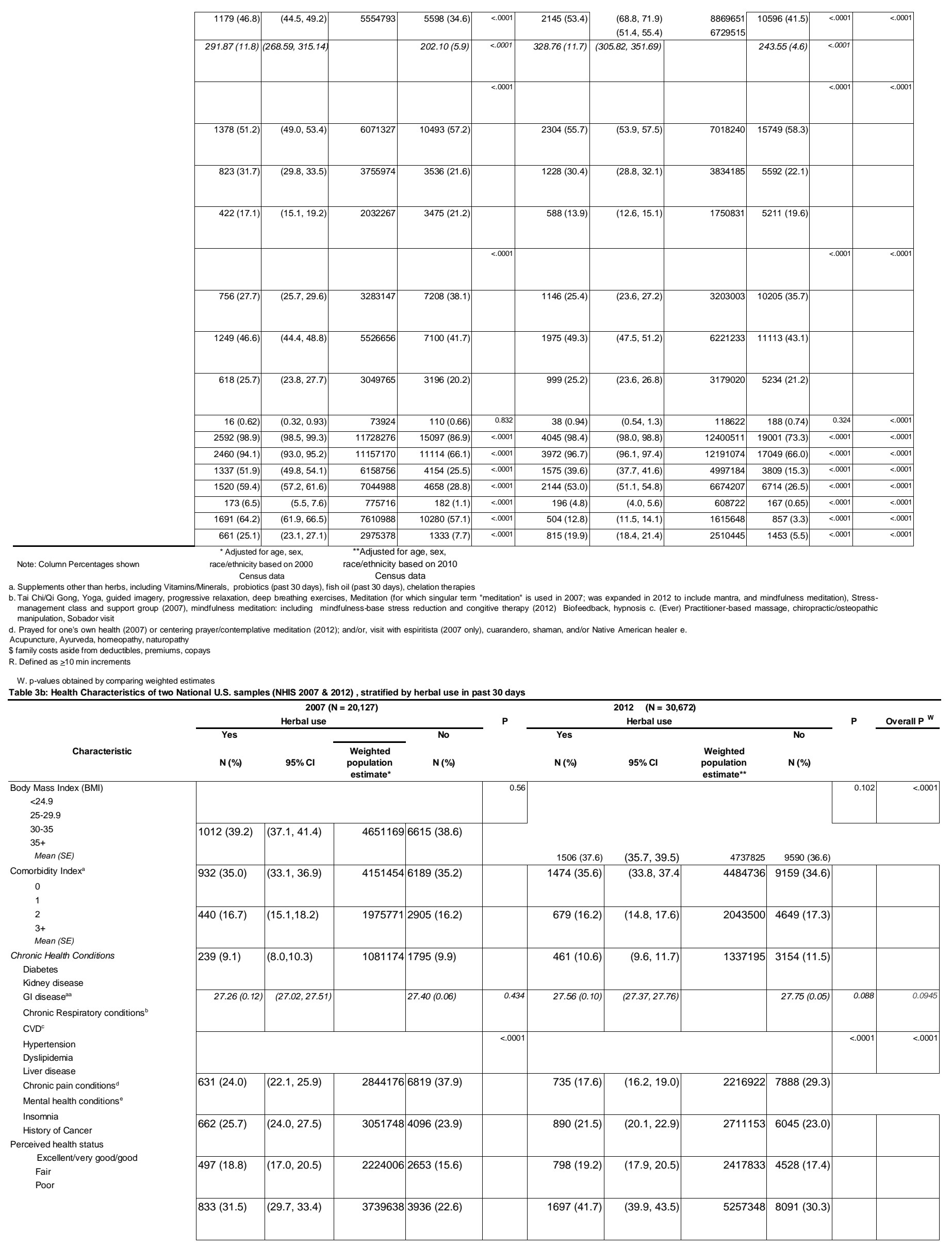




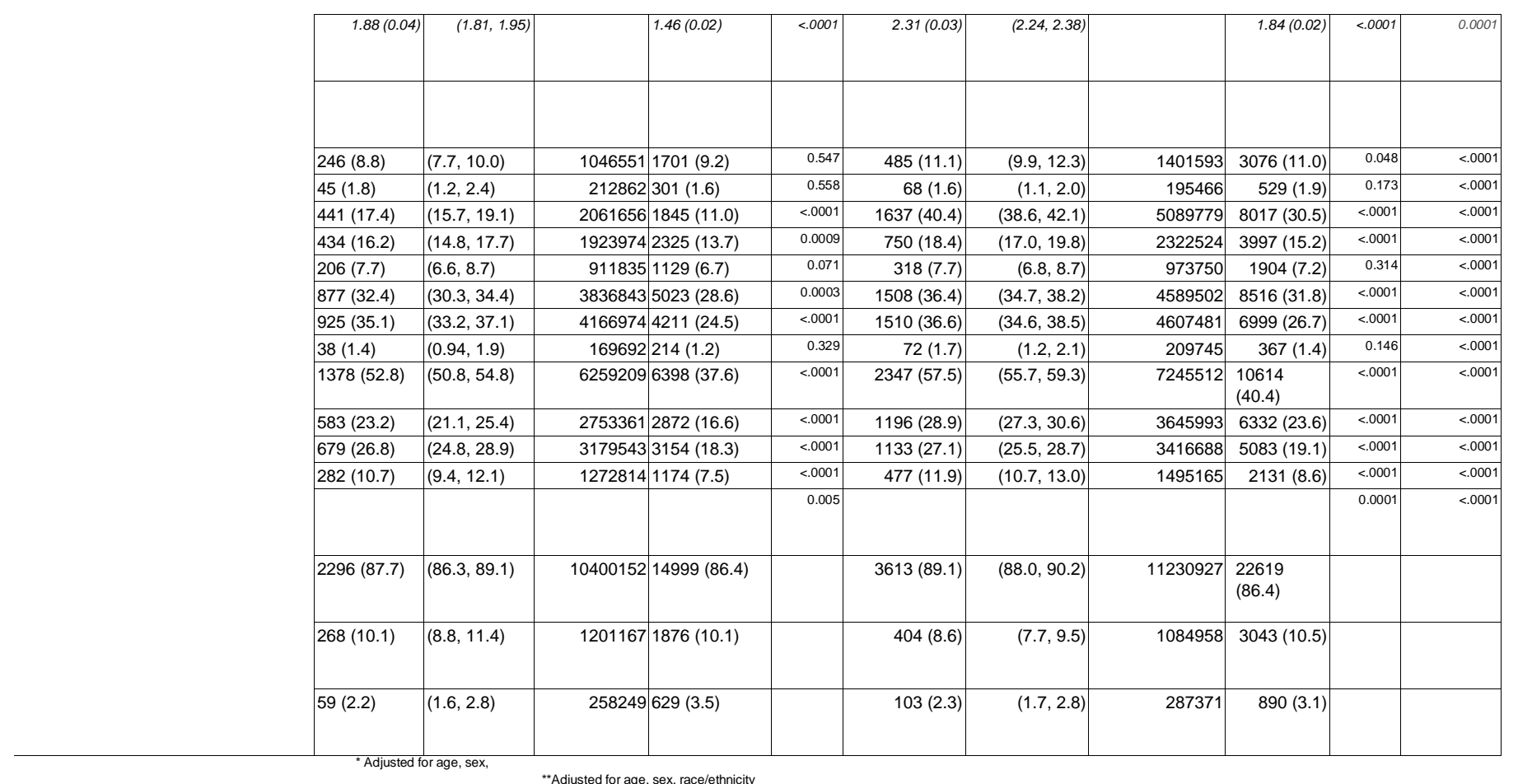

Note: Column Percentages shown race/ethnicity based on 2000 based on 2010 Census data Census data

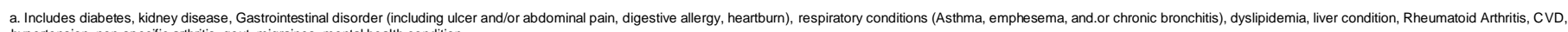
hypertension, non-specific arthritis, gout, migraines, mental health condition

aa. Includes: bowel conditions and ulcers (2007); past 12 month abdominal pain, digestive allergy, heartburn, and ever having ulcer (2012) b. Asthma,

emphysema, and/or chronic bronchitis

c. Myocardial Infarction, Heart Disease, Angina pectoris

d. Migraines (past 3 months), nonspecific arthritis, gout, non-specific joint pain for $3+$ months, Rheumatoid Arthritis

e. Depression, bipolar disorder, phobias, often anxious in past year

W. p-values obtained by comparing weighted estimates 


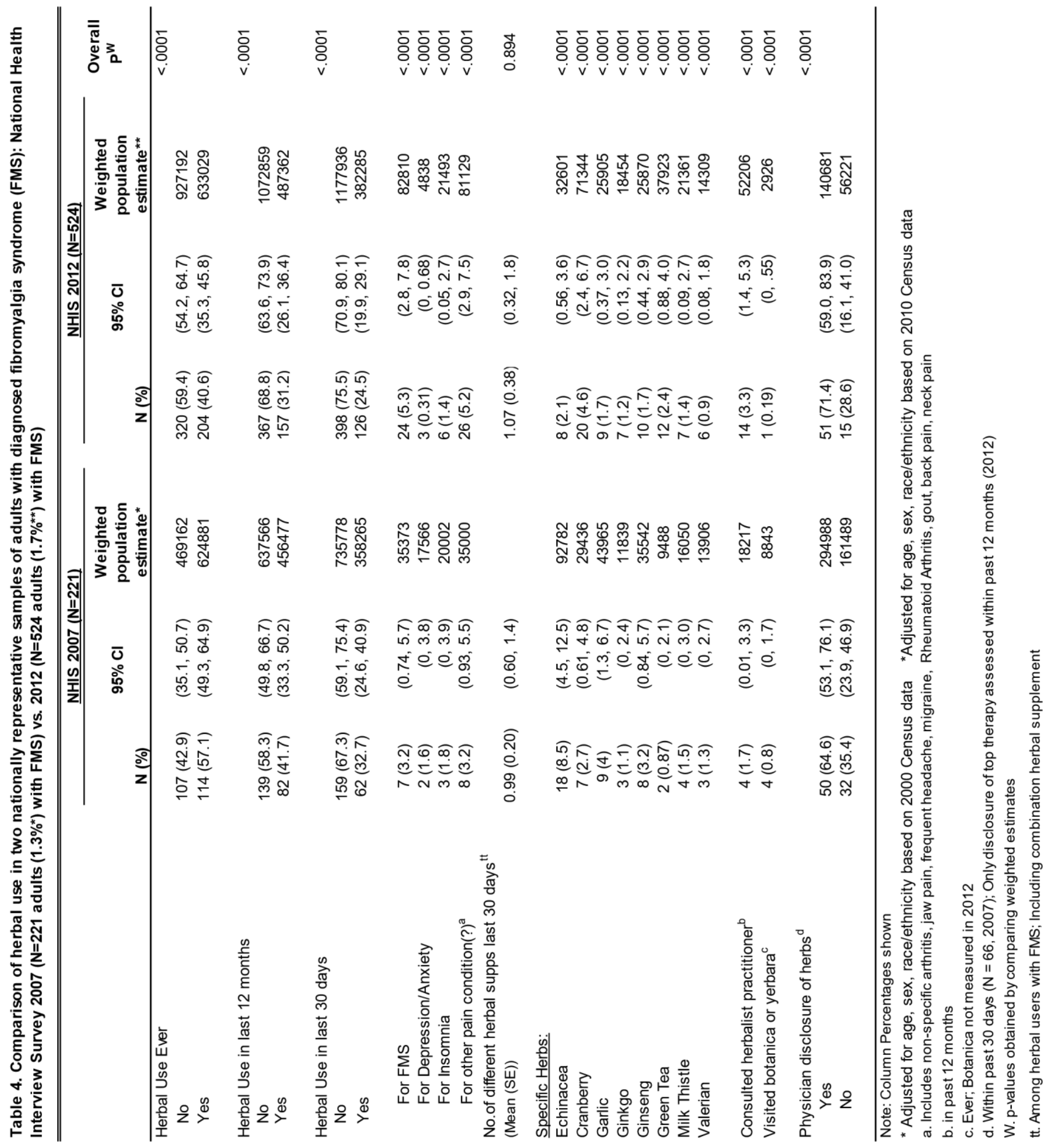




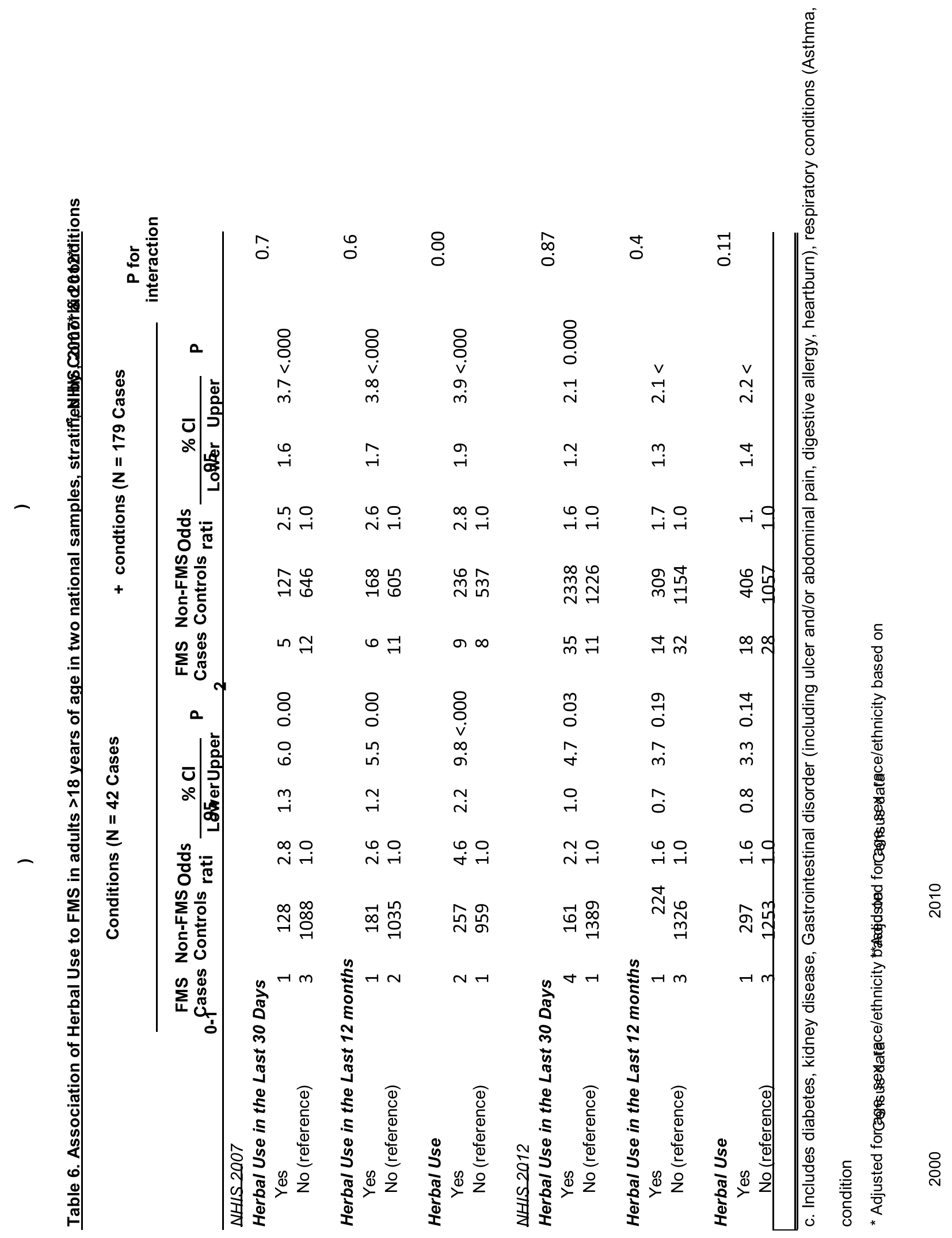




\section{Chapter 4}
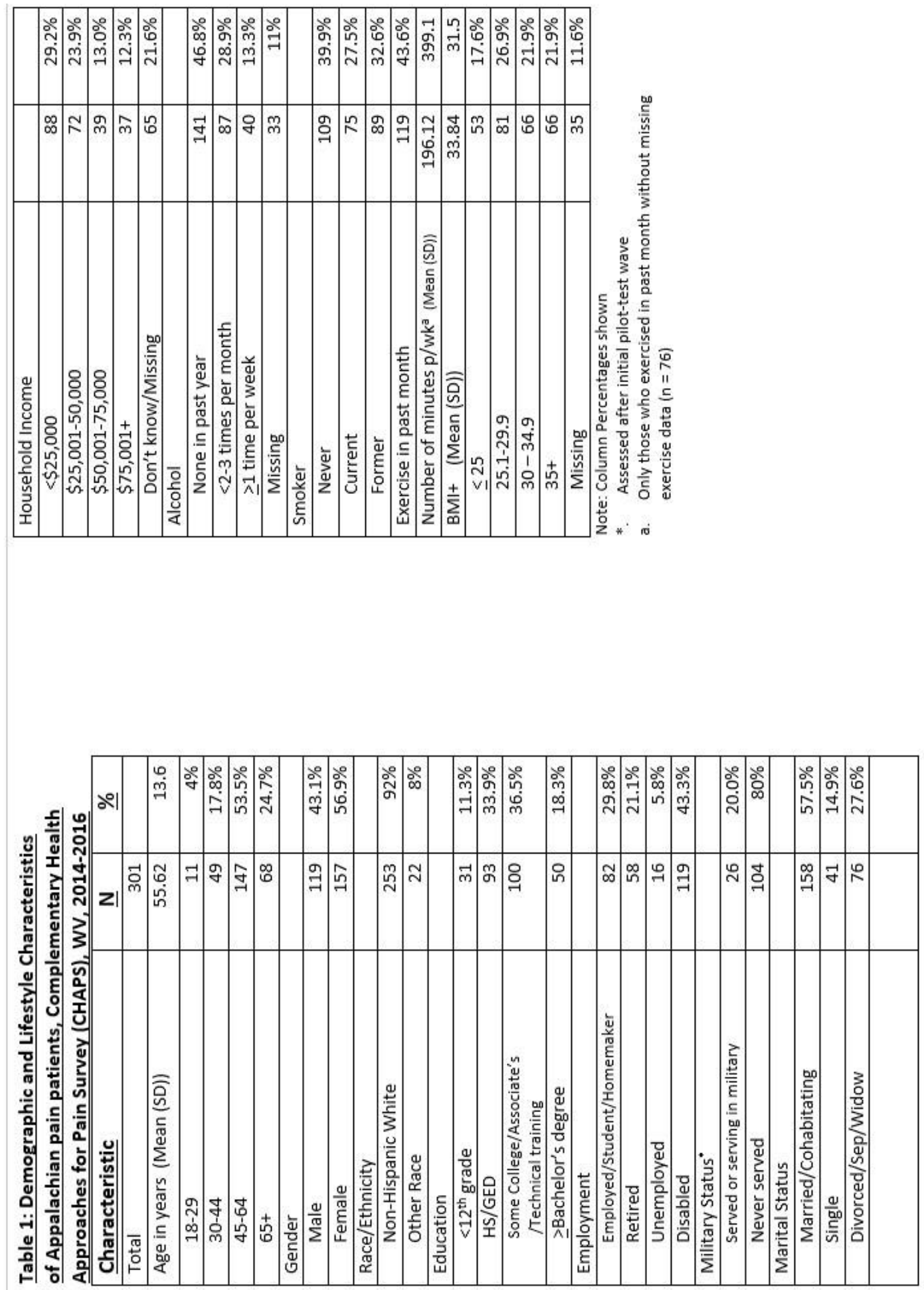
Table 2: Health Characteristics of Appalachian pain patients, Complementary Health Approaches for Pain Survey

(CHAPS), WV, 2014-2016 ( $\mathrm{N}=301$ )

\begin{tabular}{|c|c|c|}
\hline Characteristic & $\underline{\mathbf{N}}$ & $\%$ \\
\hline \multicolumn{3}{|l|}{ Chronic Pain } \\
\hline Currently experiencing & 270 & $93.4 \%$ \\
\hline Have experienced in the past & 283 & $97.6 \%$ \\
\hline \multicolumn{3}{|l|}{ Global Pain Scale $^{\mathrm{b}}$} \\
\hline Total (Mean (SD)) & 50.85 & $(20.6)$ \\
\hline Pain & 15.51 & (4.5) \\
\hline Feelings & 10.71 & (6.3) \\
\hline Clinicol Outcomes & 13.79 & (6.3) \\
\hline Activities & 12.18 & $(7.4)$ \\
\hline \multicolumn{3}{|l|}{$\begin{array}{l}\text { Prescription medication use for } \\
\text { pain management" }\end{array}$} \\
\hline None & 48 & $24.9 \%$ \\
\hline Opioids & 85 & $44.0 \%$ \\
\hline Other Rx & 60 & $31.1 \%$ \\
\hline \multicolumn{3}{|l|}{ Number of Health Conditions } \\
\hline $0-1$ & 20 & $7.2 \%$ \\
\hline 2 & 27 & $9.7 \%$ \\
\hline 3 & 39 & $14.0 \%$ \\
\hline 4 & 38 & $13.6 \%$ \\
\hline $5+$ & 155 & $55.6 \%$ \\
\hline Total ((Mean (SD)) (range) & $5.44(0-16)$ & (3.1) \\
\hline Pain syndromes ${ }^{\mathrm{c}}$ & $2.30(0-7)$ & 1.58 \\
\hline Mental Health conditions ${ }^{d}$ & $0.84(0-2)$ & 0.87 \\
\hline Injury" & $0.48(0-2)$ & 0.70 \\
\hline Other conditions $^{t}$ & $1.44(0-7)$ & 1.40 \\
\hline
\end{tabular}

Note: Column Percentages shown

- Assessed after initial pilot-test wave

b. Global Pain Scale score 0-100, with 100 indicative of greatest impact of pain upon life; Subscales Pain, feelings, clinical outcomes, and activities scores each $0-25$

c. Includes: Spine/Back/Neck pain, migraines, tension headaches, Rheumatoid Arthritis, Osteoarthritis, Temporomandibular Jaw Disorder, Knee Pain, Fibromyalgia, and/or Gout

d. Includes depression and/or anxiety

e. Includes broken bones and/or musculoskeletal injury/tissue trauma

f. Includes: Hypertension, heart disease, irritable bowel disorder, renal disorder, asthma, chronic bronchitis, diabetes, cancer, stroke, and/or chronic fatigue syndrome 


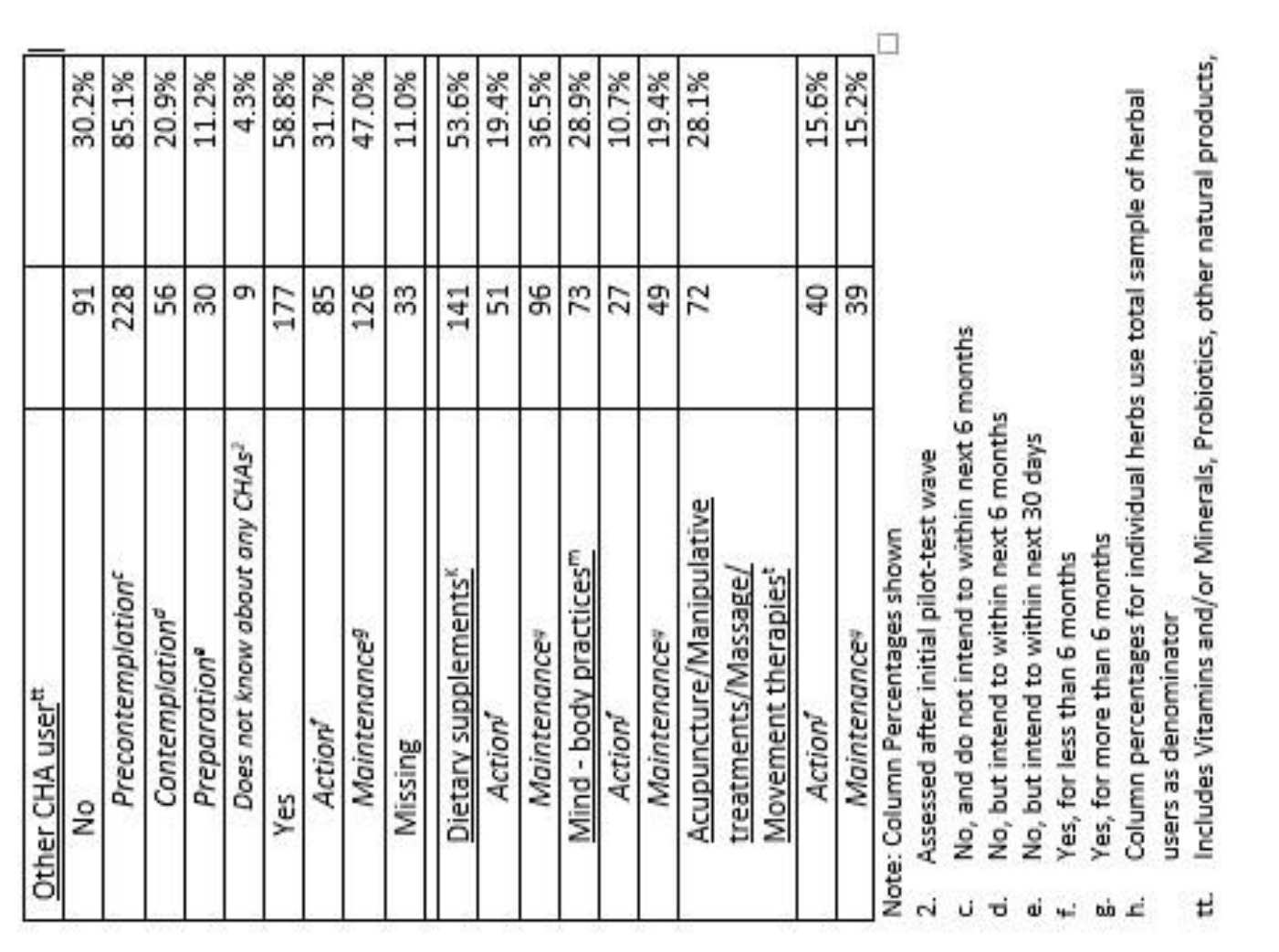

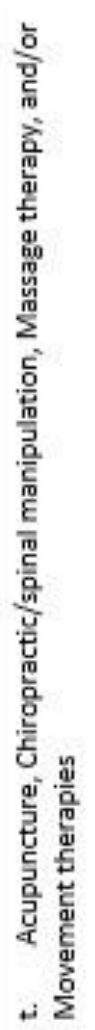

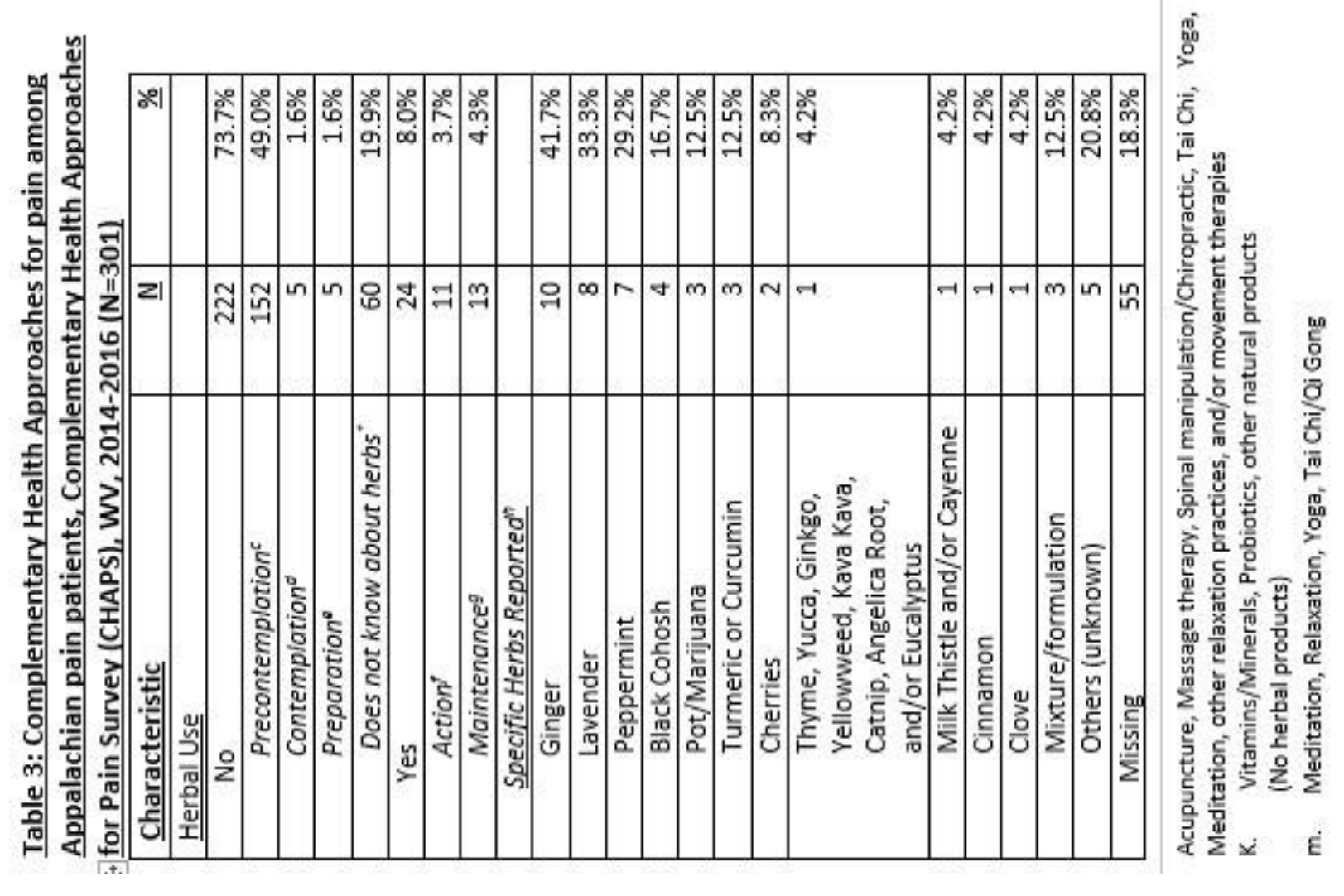




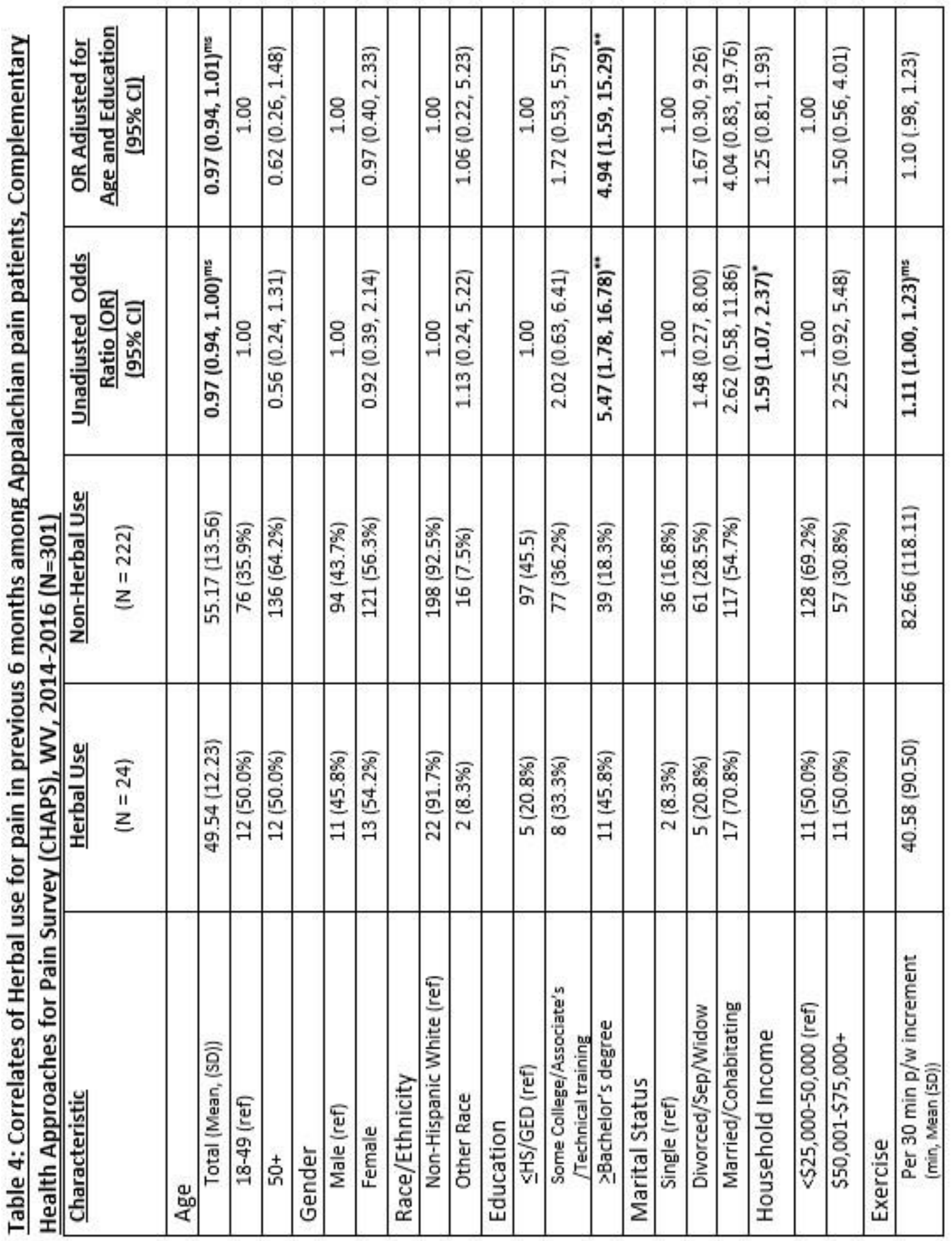




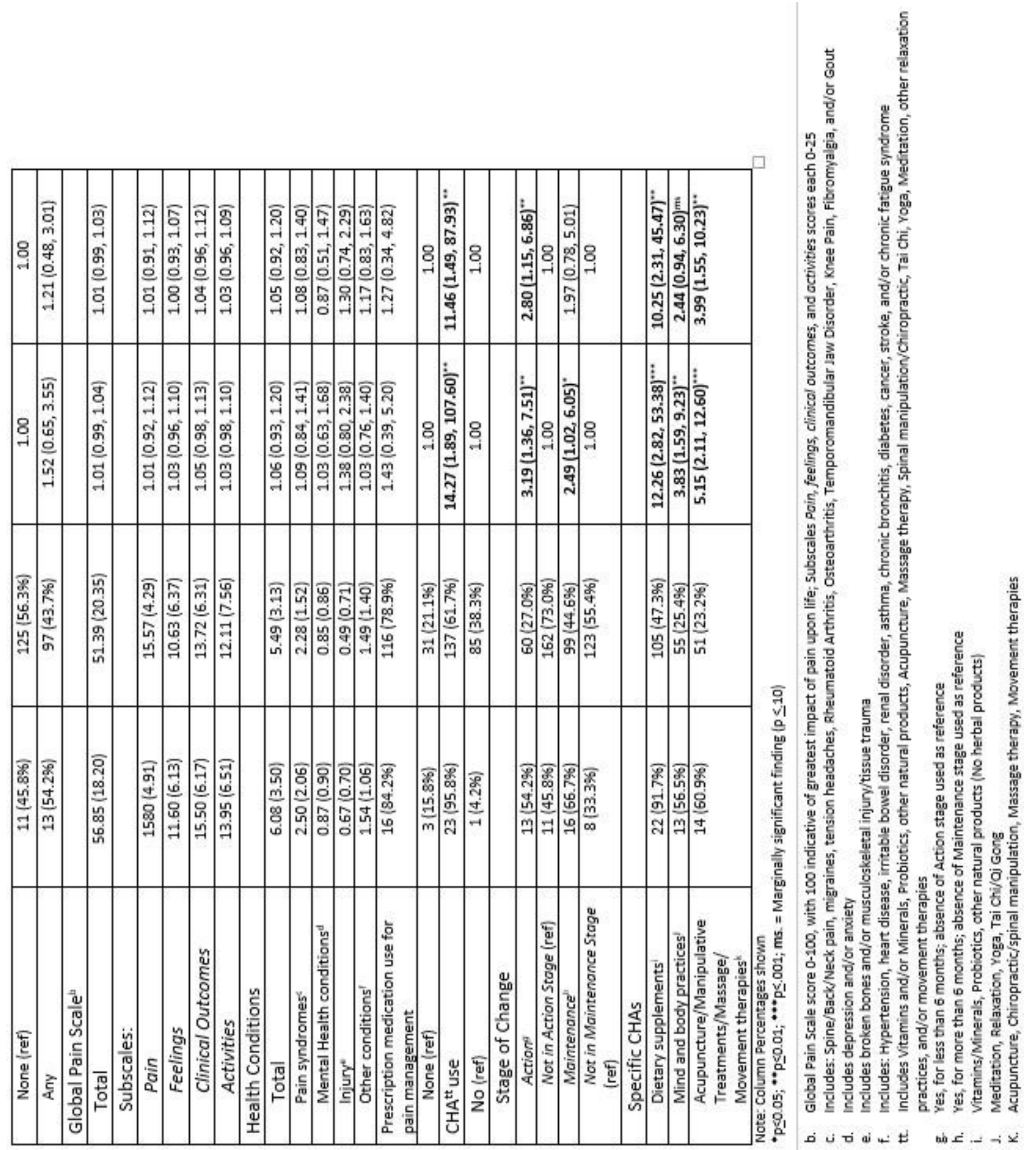




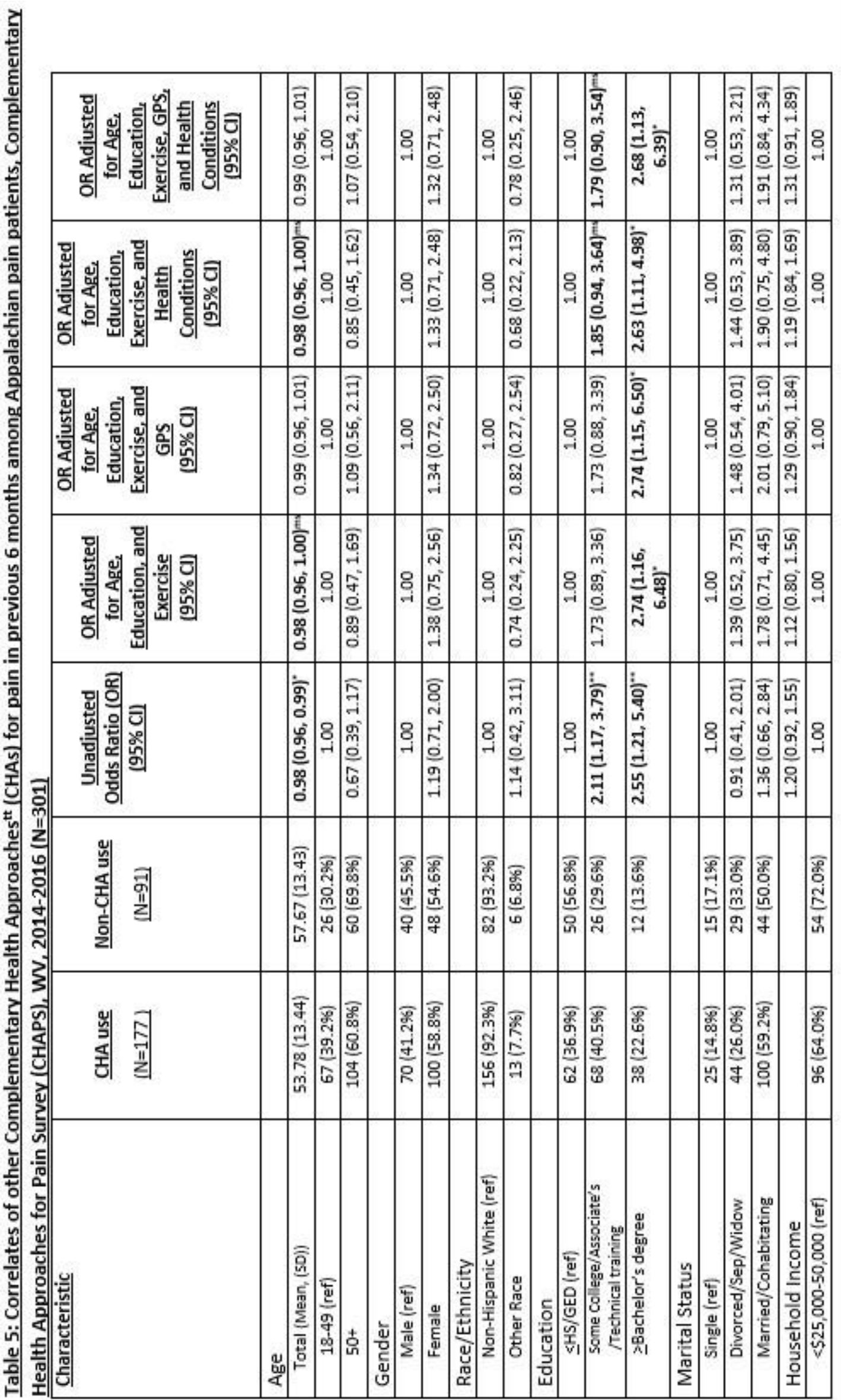




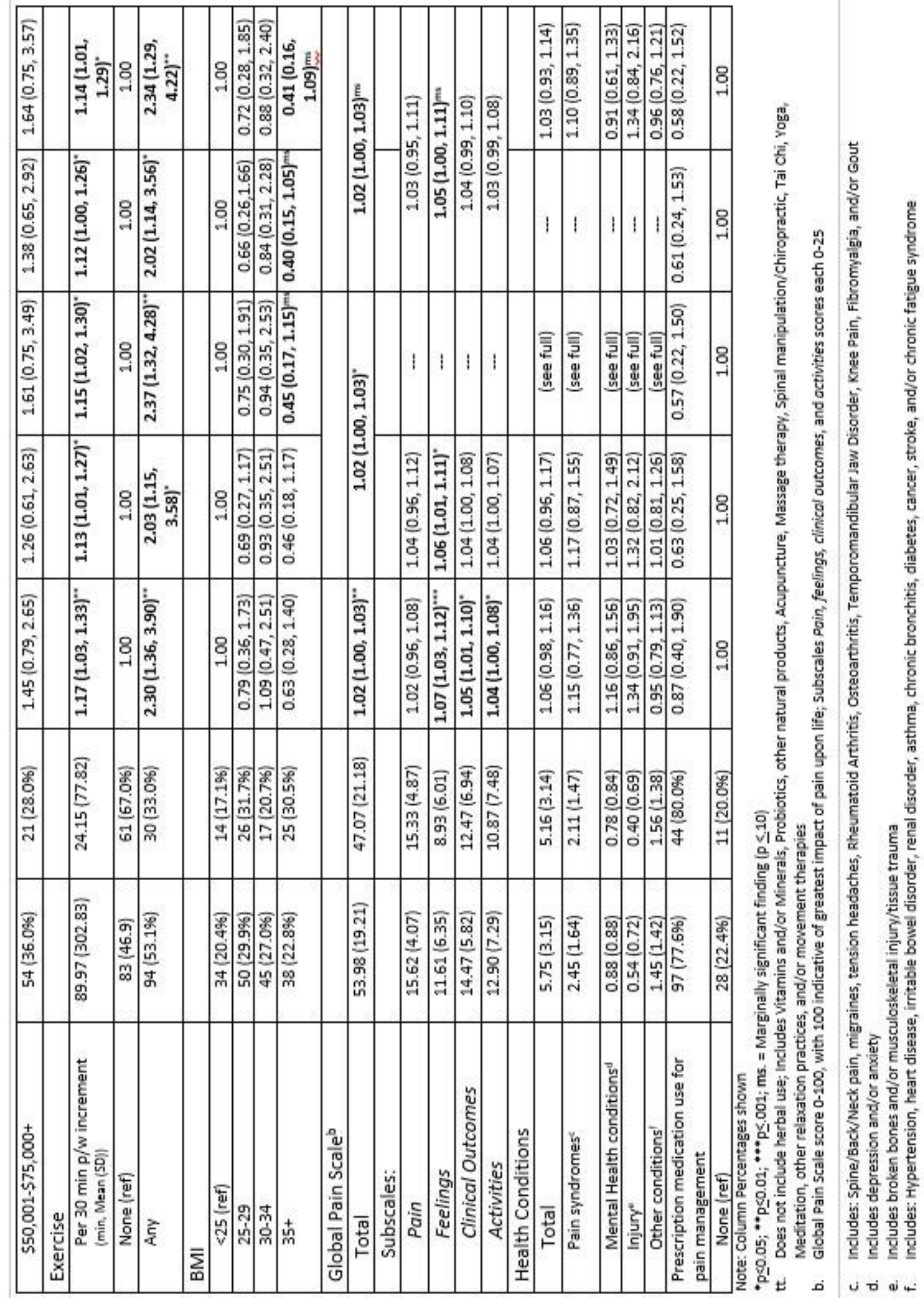




\begin{tabular}{|c|c|c|c|c|c|c|c|c|c|c|c|c|c|c|c|c|c|c|c|c|c|c|c|}
\hline & 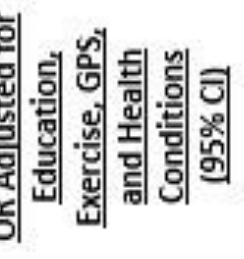 & 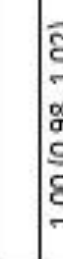 & 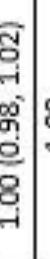 & 8 & 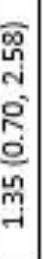 & & 8 & 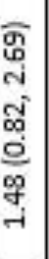 & & & 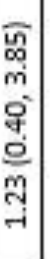 & & 8 & 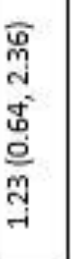 & 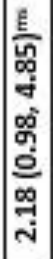 & & $\begin{array}{l}8 \\
-1\end{array}$ & 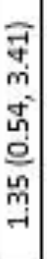 & 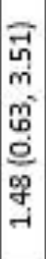 & 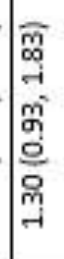 & 8 & 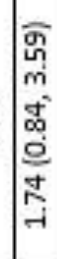 & \\
\hline & 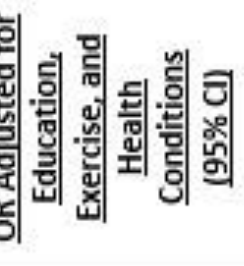 & 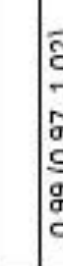 & 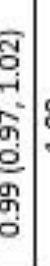 & 8 & 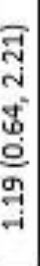 & & 8. & 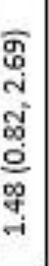 & & 8 & 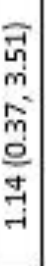 & & 8 & 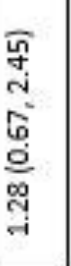 & 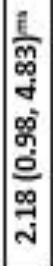 & & $\begin{array}{l}8 \\
-i \\
-i\end{array}$ & 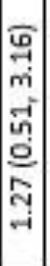 & 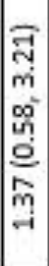 & 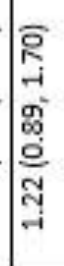 & $\underset{-i}{8}$ & 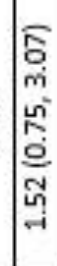 & \\
\hline & 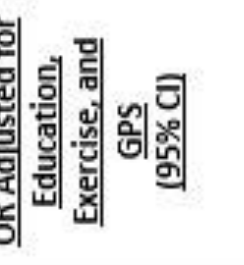 & 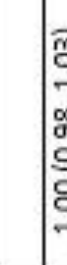 & 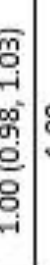 & 8. & 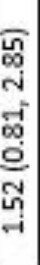 & & $\begin{array}{l} \\
8 \\
\text { - }\end{array}$ & 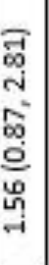 & & : & 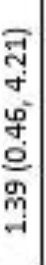 & & 8 & 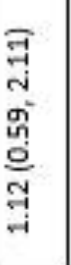 & 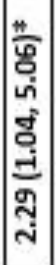 & & $\underset{-i}{8}$ & 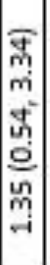 & 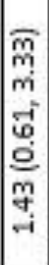 & 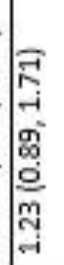 & $\stackrel{8}{\stackrel{-}{-}}$ & 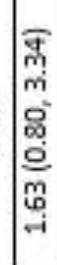 & \\
\hline & 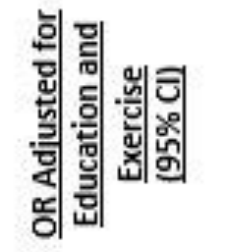 & 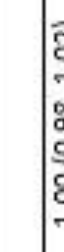 & 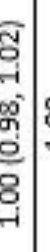 & 8 & 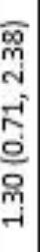 & & 8 & 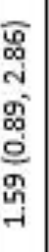 & & 8 & 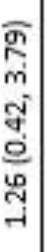 & & 8 & 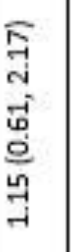 & 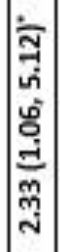 & & 8 & 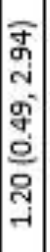 & 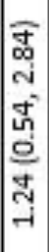 & 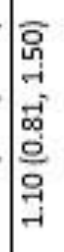 & 8 & 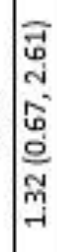 & \\
\hline & 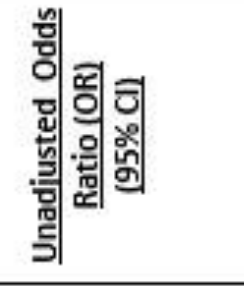 & 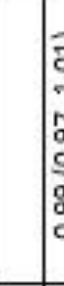 & 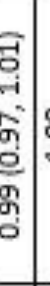 & 8 & 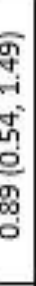 & & 8 & 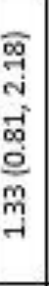 & & 8 & 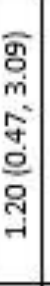 & & 8 & 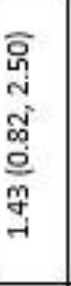 & 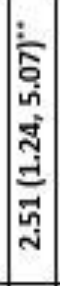 & & 8 & 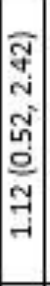 & 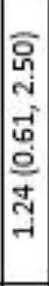 & 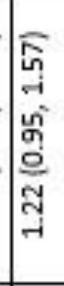 & & 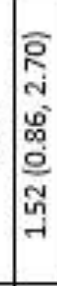 & \\
\hline & 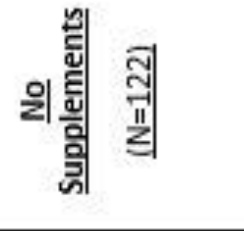 & 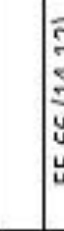 & 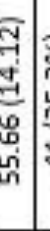 & 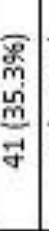 & 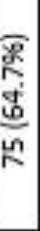 & & $\begin{array}{l}\text { 恕 } \\
\text { 守 } \\
\text { 出 } \\
\text { 员 }\end{array}$ & 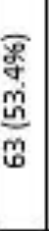 & & 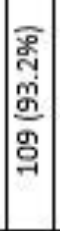 & 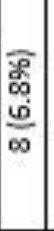 & & 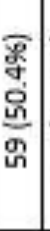 & 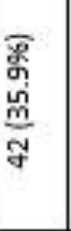 & 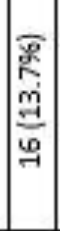 & & 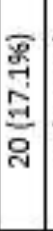 & 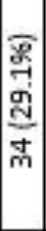 & 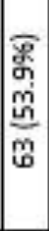 & & 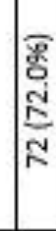 & $\begin{array}{l}\bar{s} \\
\text { o } \\
\text { d } \\
\text { s } \\
\text { s. }\end{array}$ & \\
\hline & 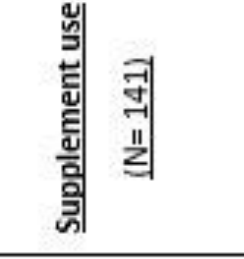 & مَ & 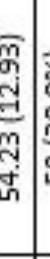 & 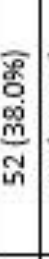 & 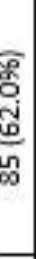 & & 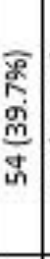 & 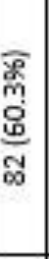 & & 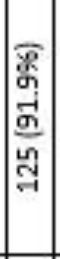 & 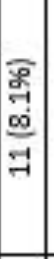 & & 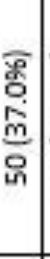 & 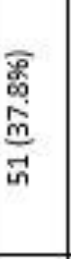 & 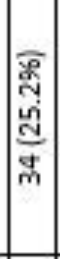 & & 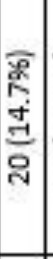 & 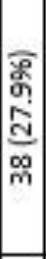 & 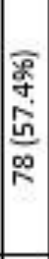 & & 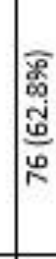 & 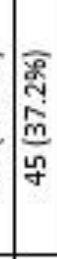 & \\
\hline & & 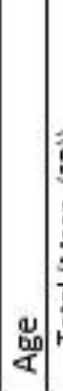 & 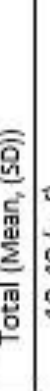 & 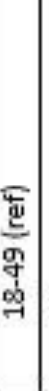 & 莒 & 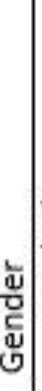 & $\frac{\frac{\omega}{\frac{u}{2}}}{\frac{m}{2}}$ & 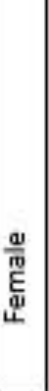 & 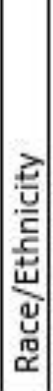 & 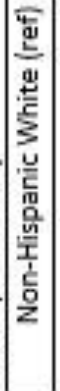 & 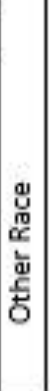 & 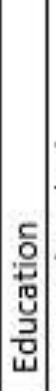 & 密 & 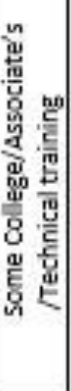 & 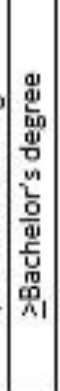 & 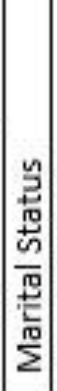 & 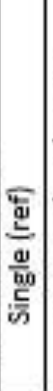 & 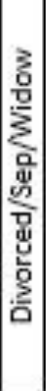 & 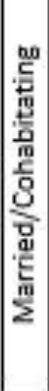 & 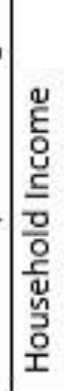 & 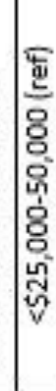 & 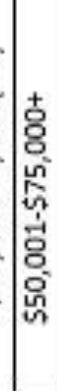 & 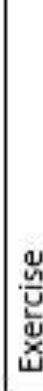 \\
\hline
\end{tabular}




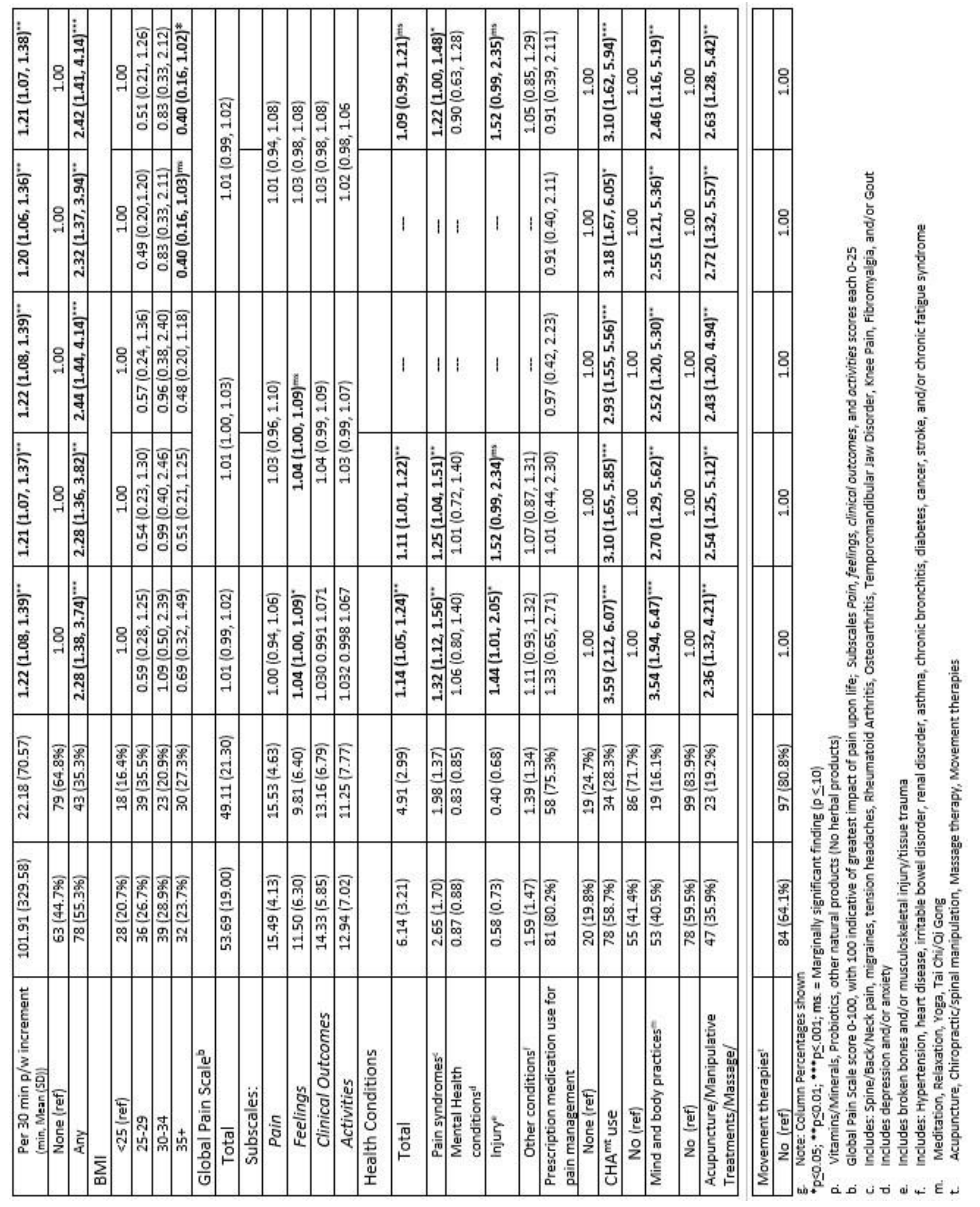




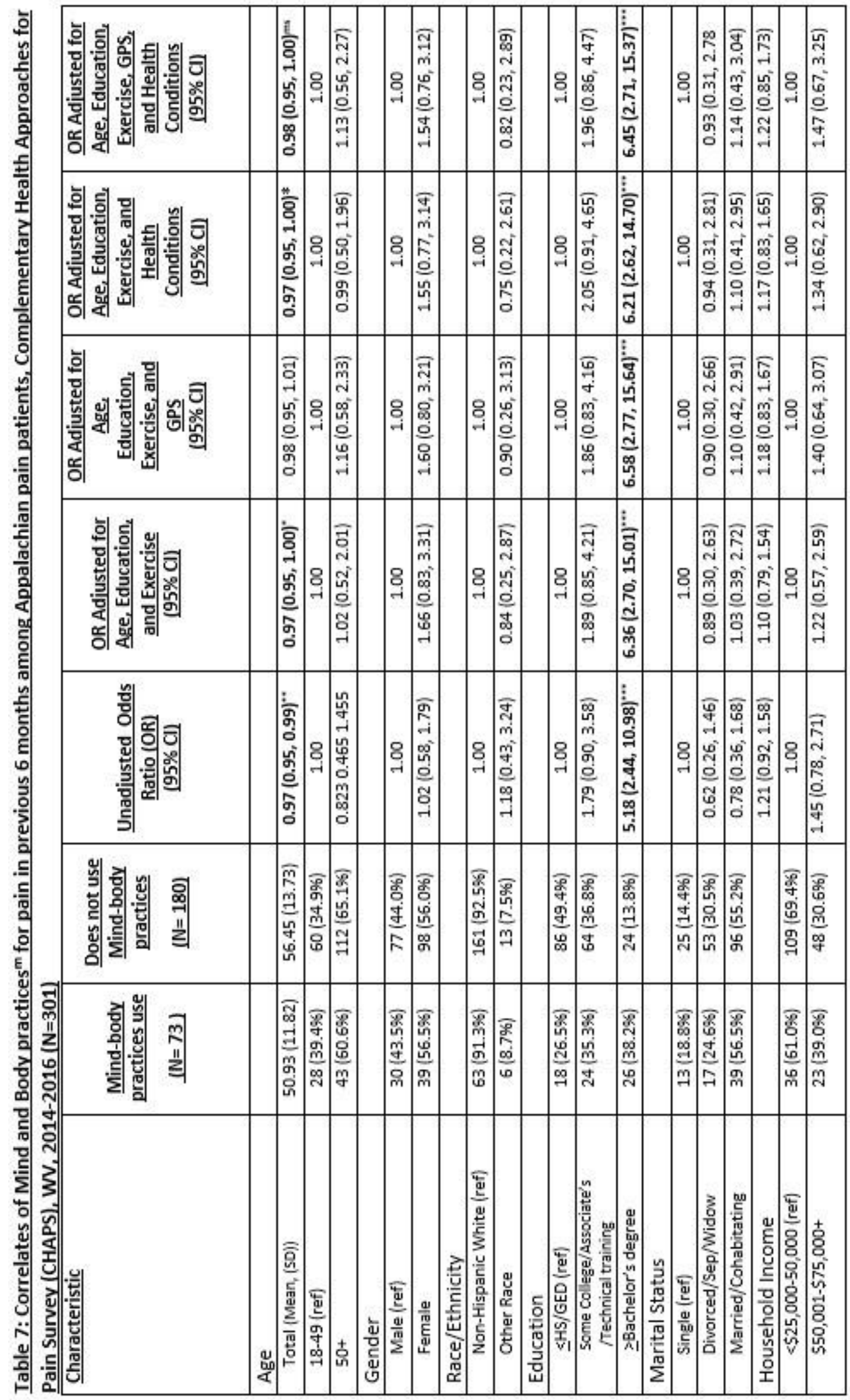




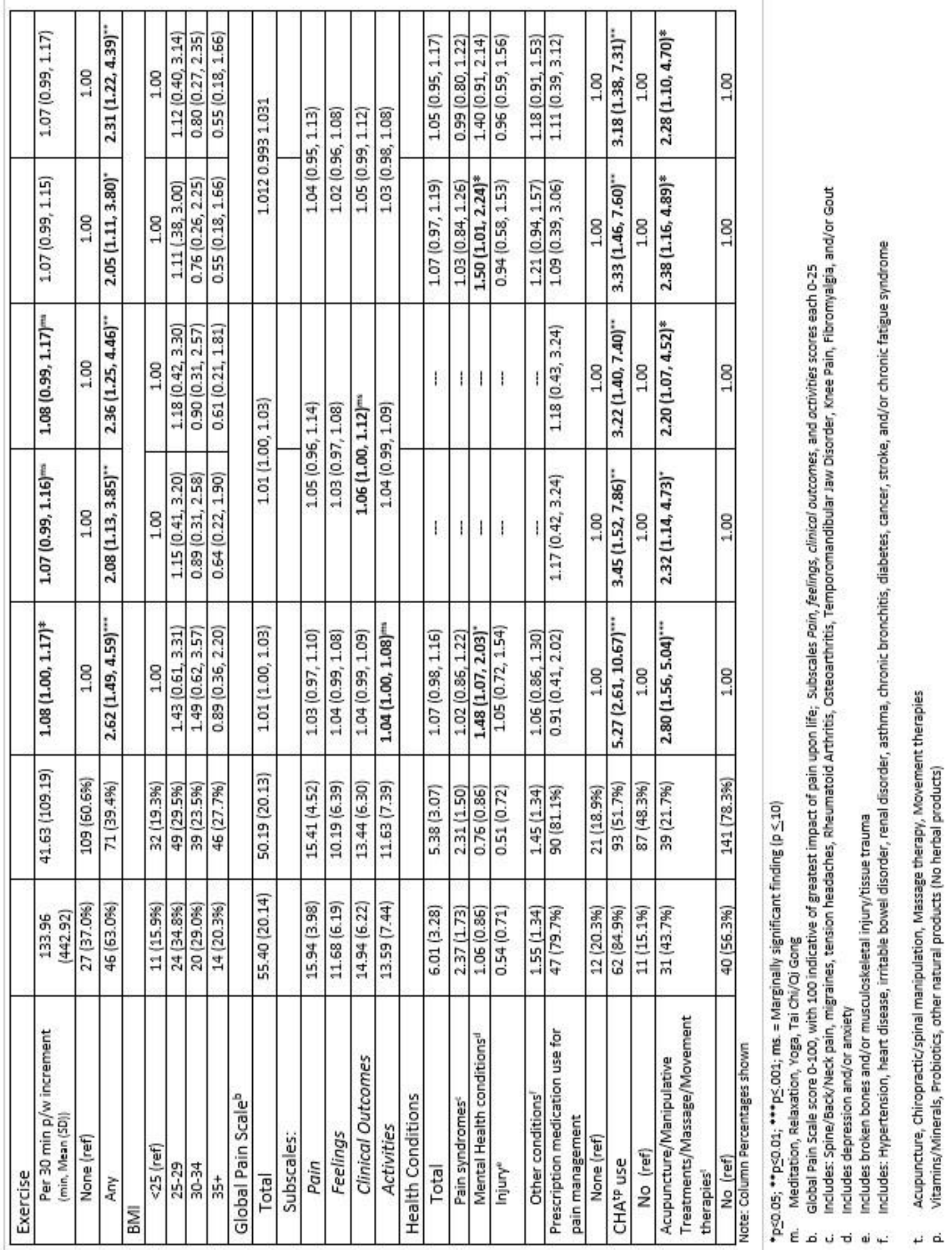




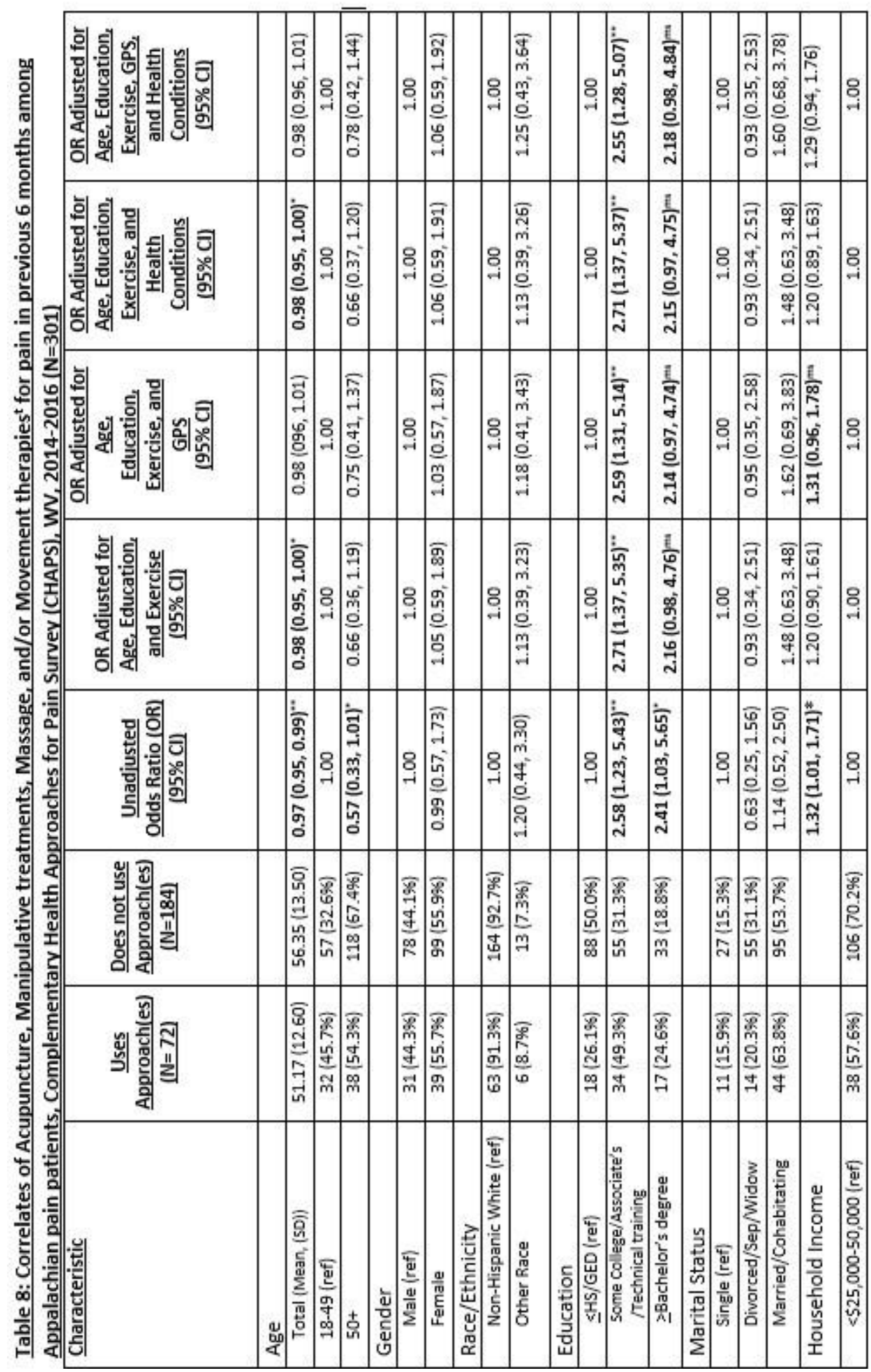




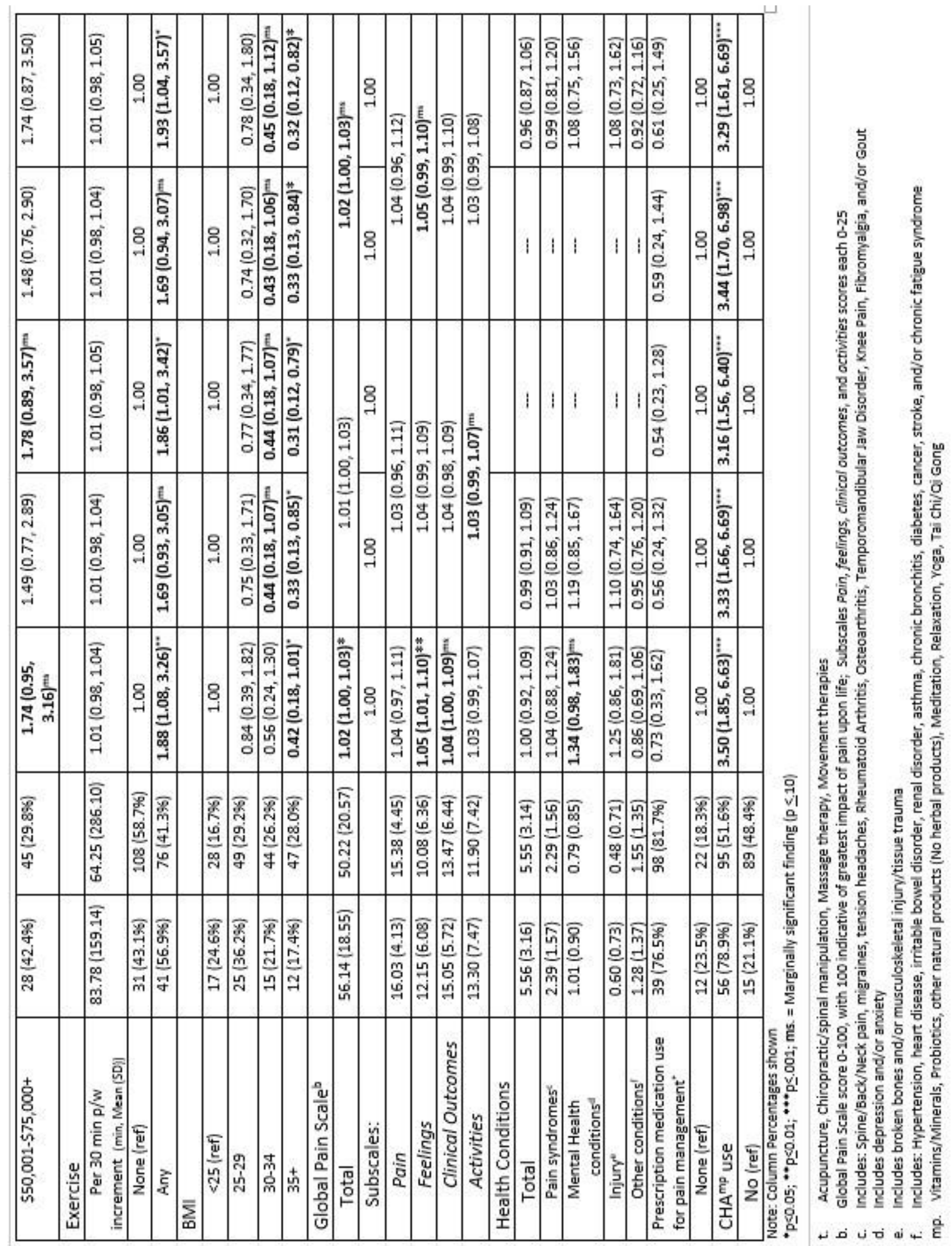




\section{Images}

\section{Chapter 1}

Image 1: Current harvesting of medicinal plants in West Virginia

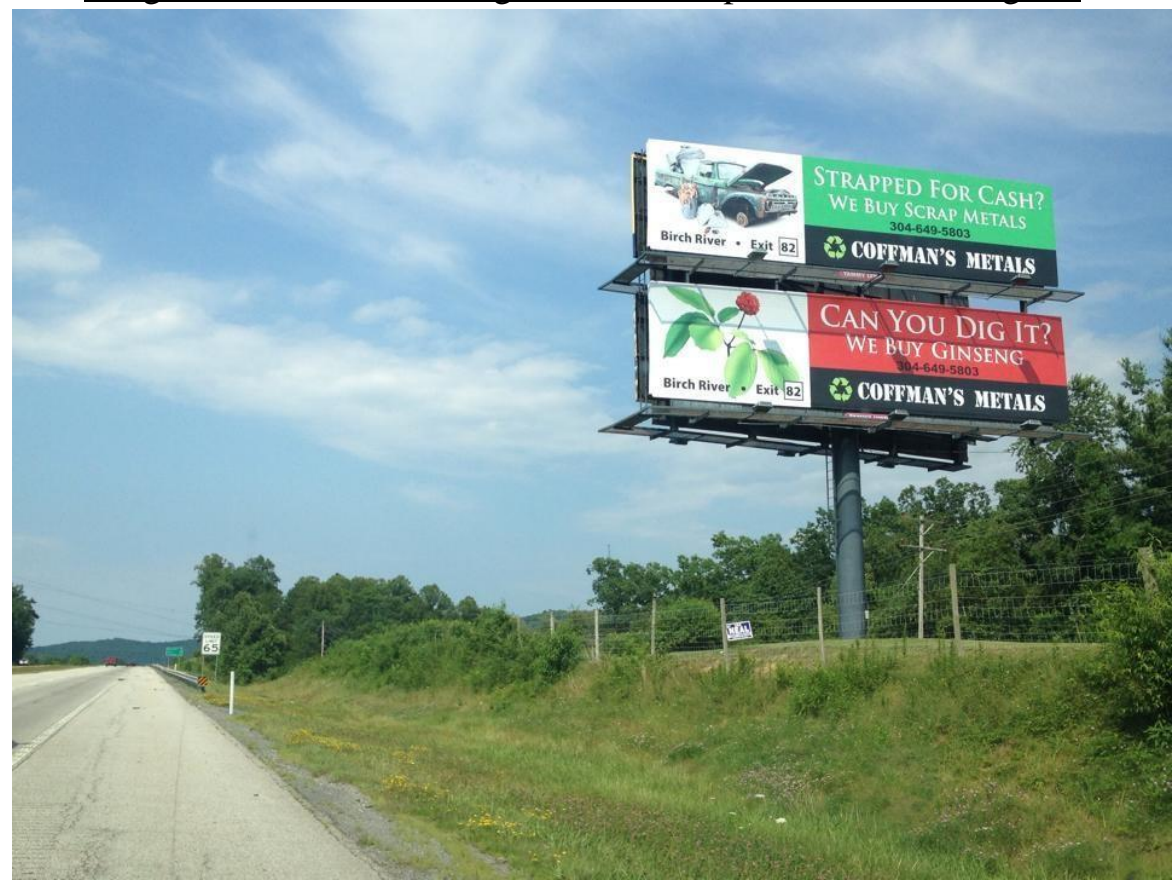

(Photo taken by author July 10, 2014 near Hookersville, WV)

\section{Chapter 3}

Image 1: Percentage of adults who used nonvitamin, nonmineral dietary supplements in the past 12 months, by region: United States, 2012

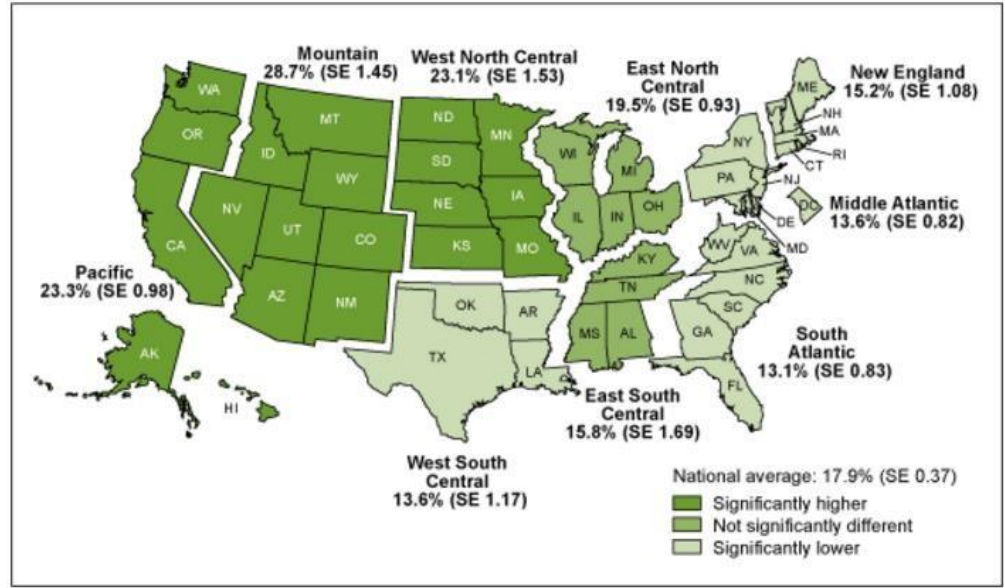

NOTES: SE is standard error. Significance is evaluated at the 0.05 level.

SOURCE: CDC/NCHS, National Health Interview Survey, 2012. 


\section{Figures}

\section{Chapter 1}

Figure 1: Taxonomy of Folk Illnesses in Appalachia

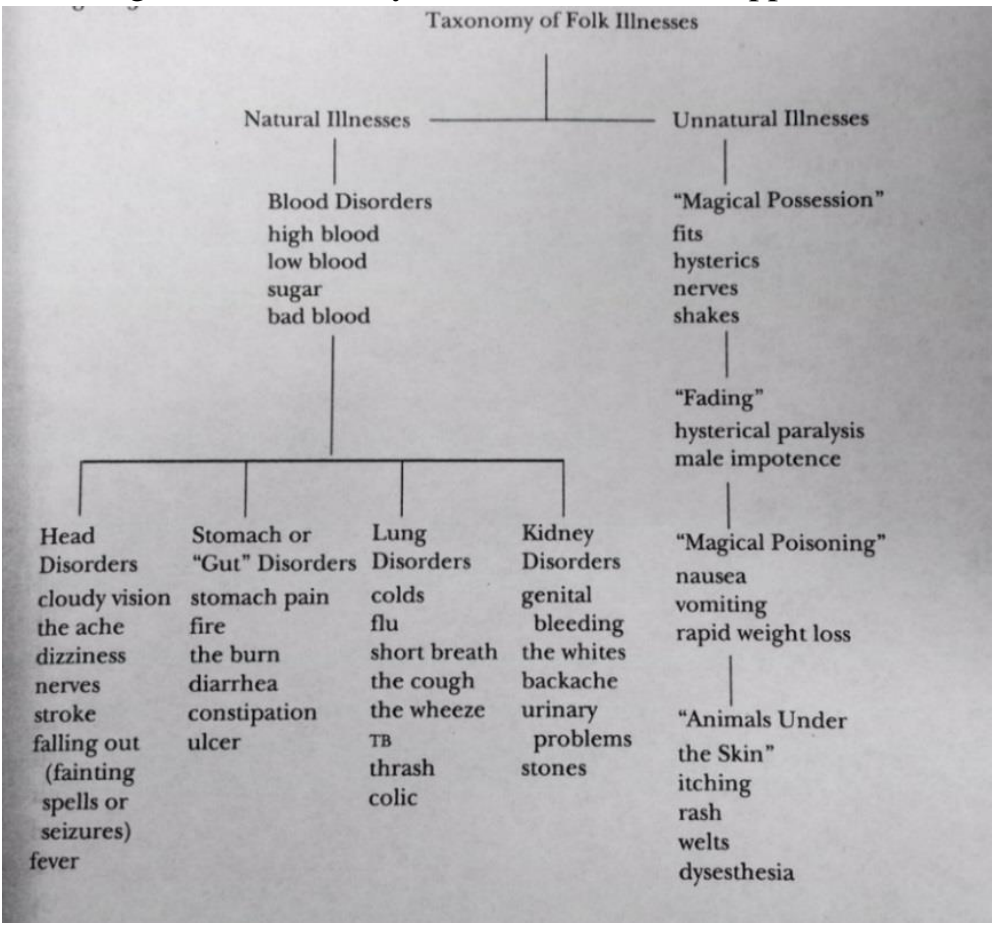

(Matthews et al 1992)

Figure 2: Evolution of Folk Medicine in Appalachia

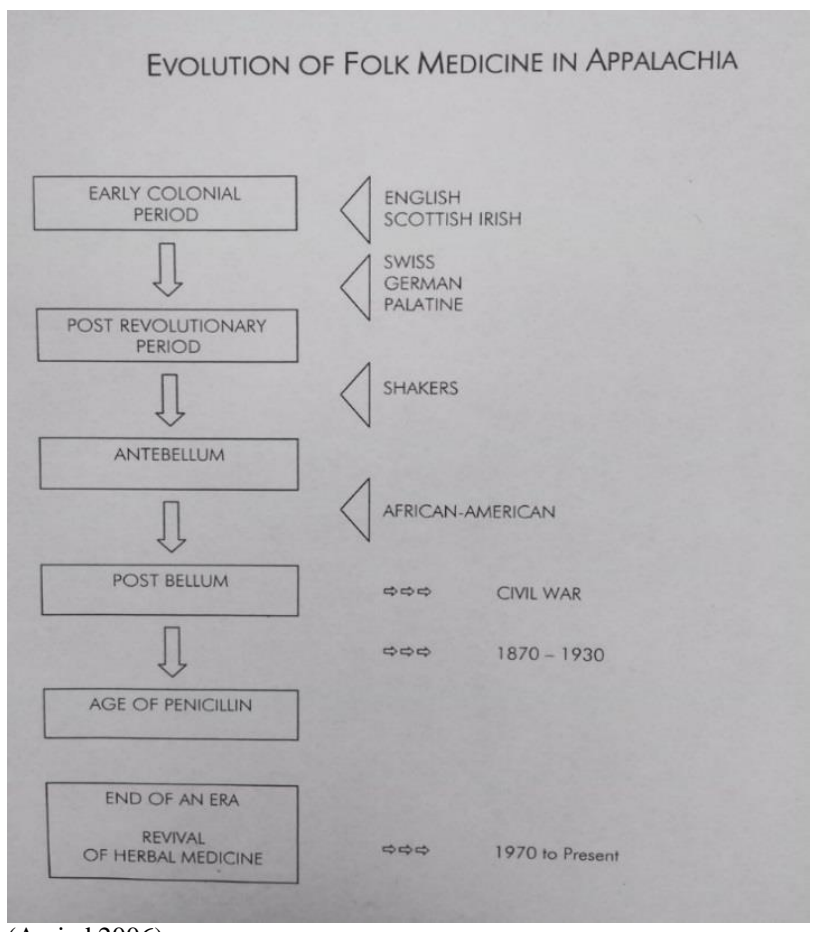

(Amjad 2006) 
Figure 3: Information Flow Pathways of Efficacy Research for use of Herbal Product vs. Prescription Drug

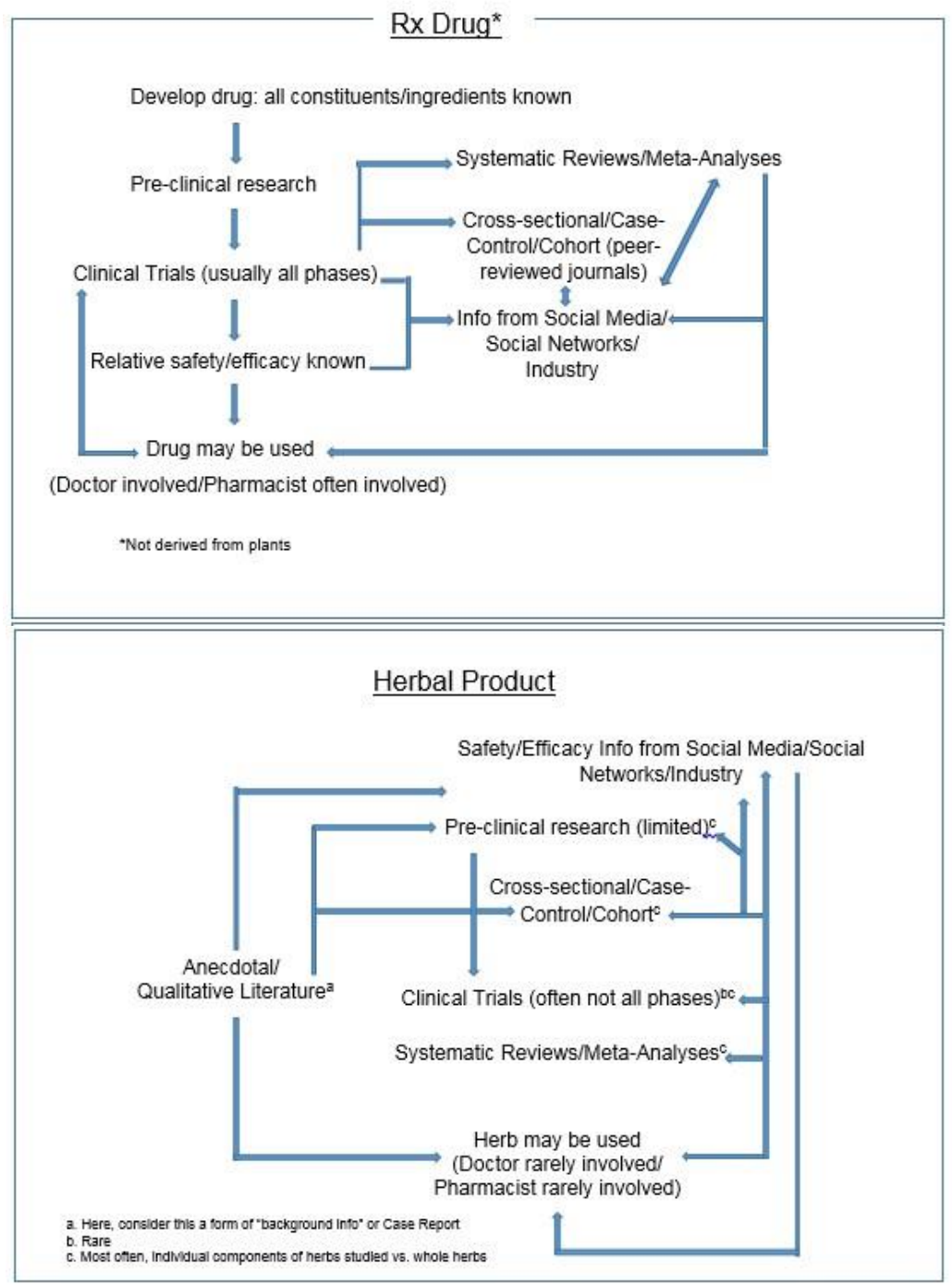


Figure 4. Conceptual Model of the Relationships between Chronic Pain, Inflammation, and Herbal Medicine

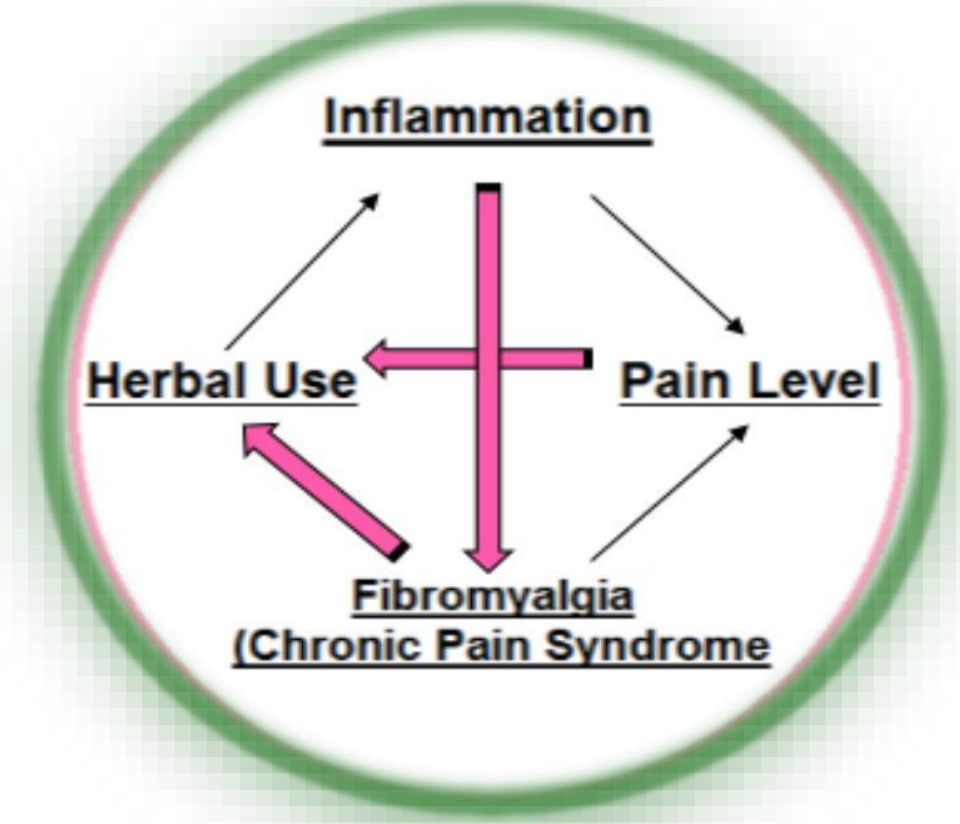




\section{Chapter 3}

Figure 1. Study Flow for two NHIS datasets
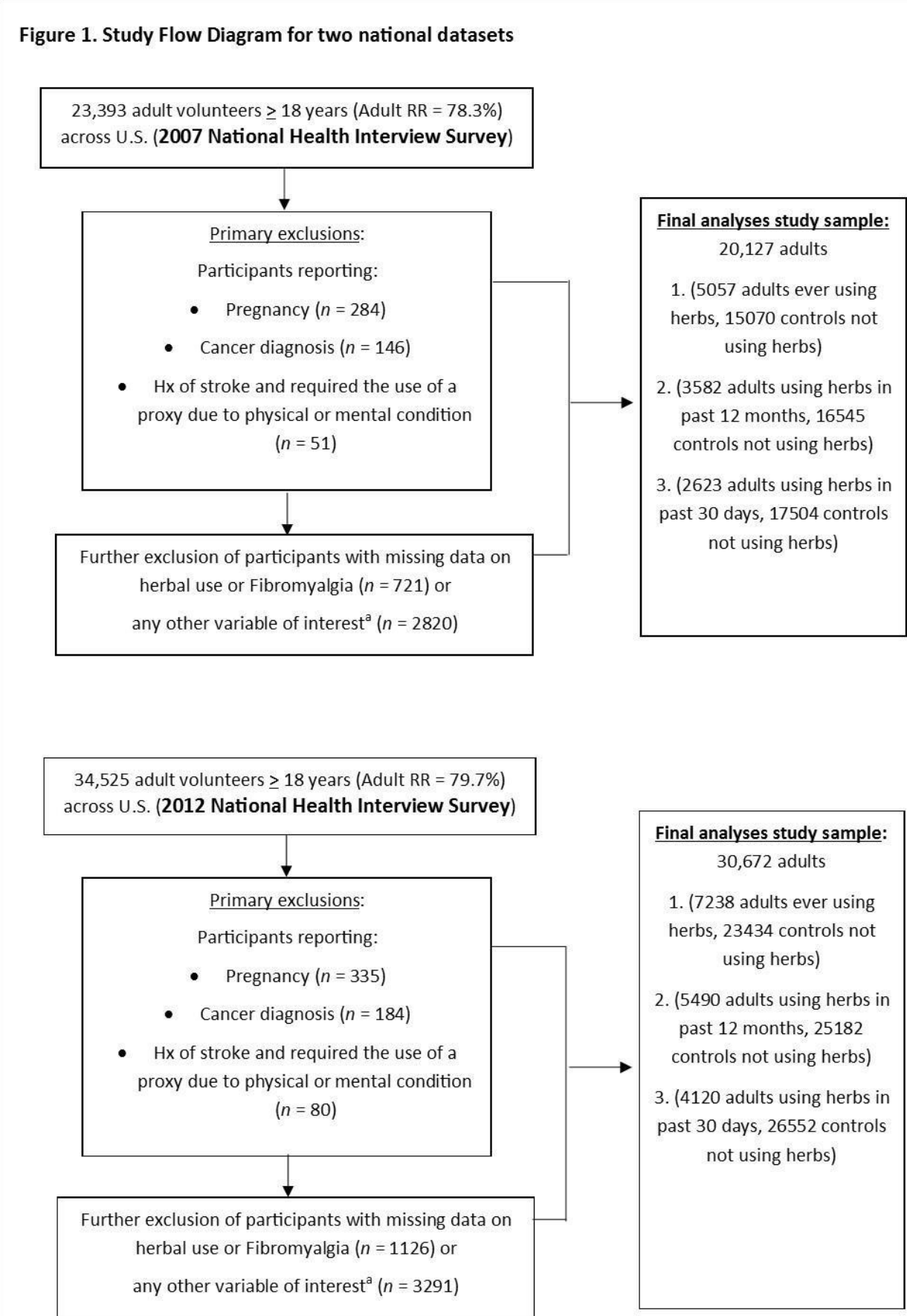

a: with exception of income, which contained $\geq 10 \%$ of missing values in both datasets; these were considered separate categories for analysis

\section{Chapter 4}


Figure 1. The Transtheoretical Model's Six Stages of Change

\section{Prochaska's Six Stages of Change}

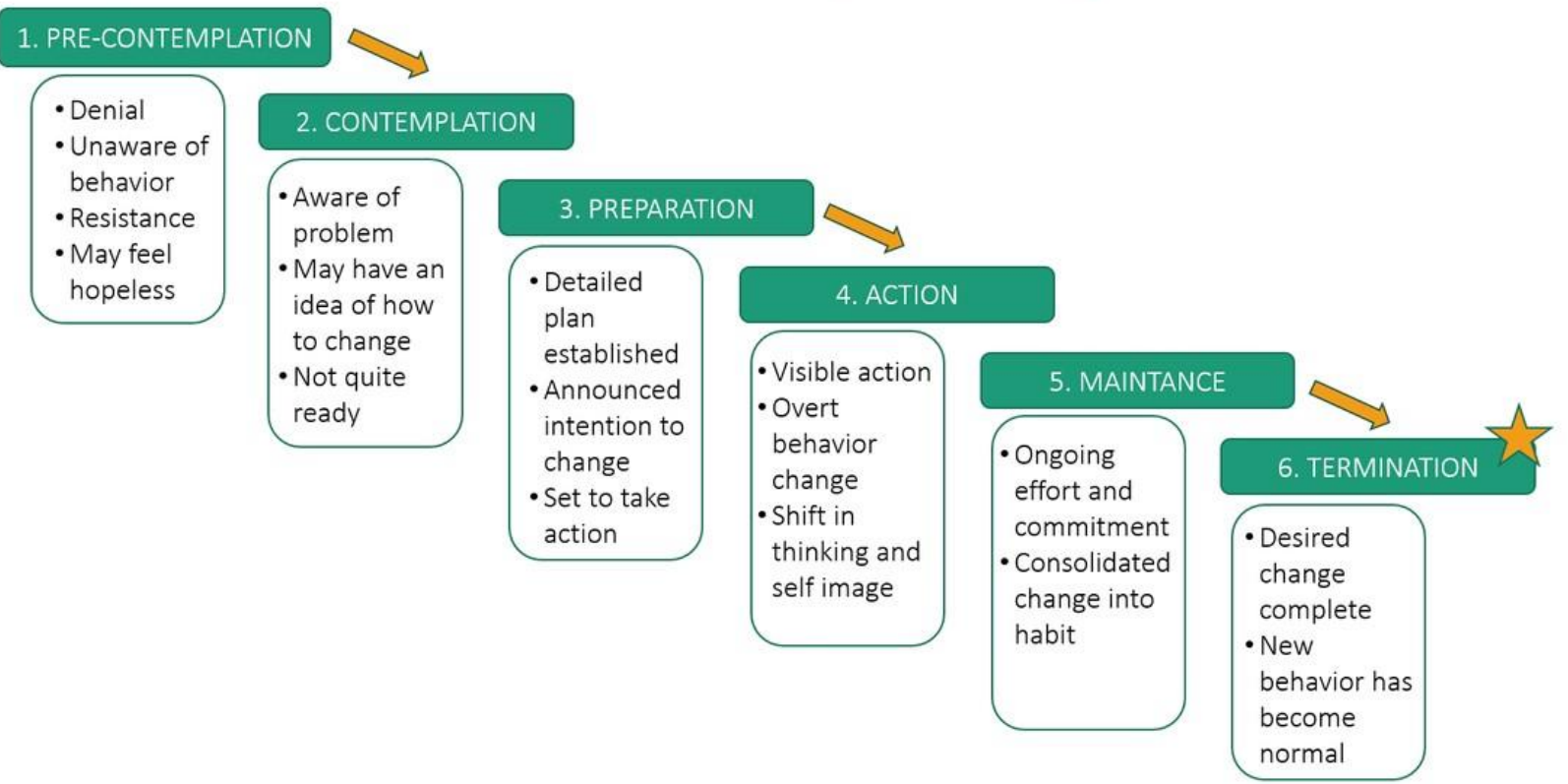


Figure 2. Flow diagram for the CHAPS 2014-2016

Figure 2. Flow diagram. CHAPS Survey Validation, selection of participant sampling sites, \& final sample

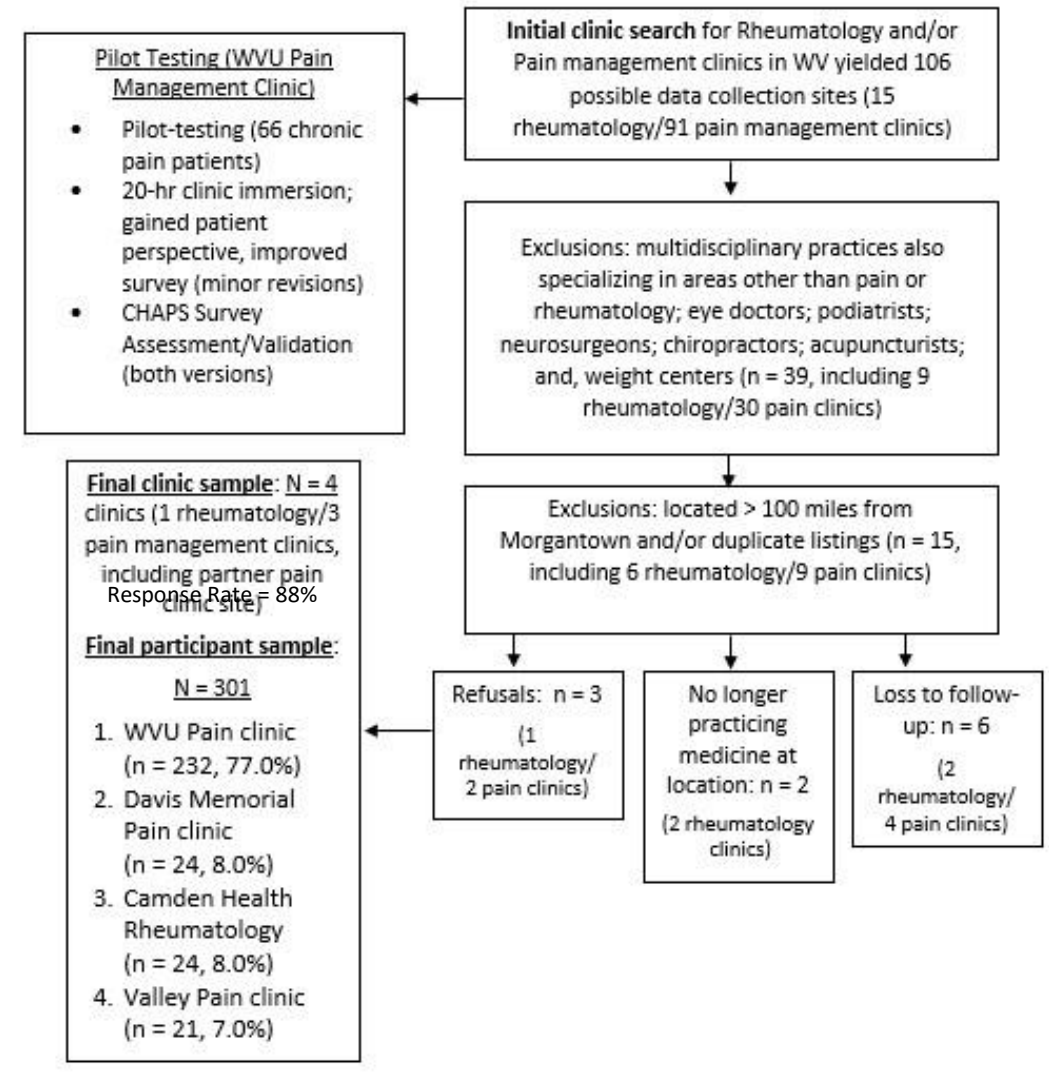




\section{Appendix A.}

\begin{tabular}{|c|c|c|c|}
\hline \multirow{2}{*}{\multicolumn{4}{|c|}{$\begin{array}{l}\text { Search Results for Co } \\
\text { FMS/CFS/Chronic Pain }\end{array}$}} \\
\hline & & & \\
\hline \multirow{2}{*}{\multicolumn{3}{|c|}{$\begin{array}{l}\text { Results: 206; search performed 10/10/14 using search terms "herb" "herbal" } \\
\text { "botanical" "fibromyalgia" in Cochrane Summaries }\end{array}$}} & GUIDE \\
\hline & & & \\
\hline \multicolumn{3}{|c|}{$\begin{array}{l}\text { Relevant studies to non-cancerous chronic pain relating to fibromyalgia or } \\
\text { chronic fatigue and herbs manually pulled: } 22\end{array}$} & $\begin{array}{l}\text { herbs mentioned } \\
\text { treatment }\end{array}$ \\
\hline & & & $\begin{array}{l}\text { non-herbal } \\
\text { therapies } \\
\text { as treatment }\end{array}$ \\
\hline Description & $\begin{array}{l}\text { Type of herb or } \\
\text { intervention }\end{array}$ & Results & $\mathbf{N}$ (if clear) \\
\hline $\begin{array}{l}\text { Traditional Chinese medicinal herbs } \\
\text { for the treatment of idiopathic } \\
\text { chronic fatigue and chronic fatigue } \\
\text { syndrome;"Traditional Chinese } \\
\text { medicinal herbs for chronic } \\
\text { fatigue";CD006348;http://su } \\
\text { mmaries.cochrane.org/CD006 } \\
\text { 348/DEPRESSN_traditionalchinese- } \\
\text { medicinal-herbs-forchronic-fatigue }\end{array}$ & $\begin{array}{l}\text { No studies met } \\
\text { inclusion criteria } \\
\text { and specific herbs } \\
\text { used were not } \\
\text { specified }\end{array}$ & & \\
\hline $\begin{array}{l}\text { Anticonvulsants for } \\
\text { fibromyalgia;"Anticonvulsant s for } \\
\text { fibromyalgia";CD010782; http } \\
\text { ://summaries.cochrane.org/C } \\
\text { D010782/MUSKEL_anticonv } \\
\text { ulsants-for-fibromyalgia }\end{array}$ & anti-epileptic drug & $\begin{array}{l}\text { reliable conclusions } \\
\text { for pregabalin only }\end{array}$ & $\begin{array}{l}\text { This is an original } \\
\text { review which was } \\
\text { withdrawn in } 2009 \\
\text { and replaced by the } \\
\text { same group's } \\
\text { "Antiepileptic } \\
\text { drugs for } \\
\text { neuropathic pain } \\
\text { and fibromyalgia" } \\
\text { in } \\
2013\end{array}$ \\
\hline $\begin{array}{l}\text { Exercise for treating fibromyalgia } \\
\text { syndrome;"Exercise for } \\
\text { fibromyalgia";CD003786;http } \\
\text { ://summaries.cochrane.org/C } \\
\text { D003786/MUSKEL_exercise } \\
\text {-for-fibromyalgia }\end{array}$ & exercise & $\begin{array}{l}\text { There is 'gold' level } \\
\text { evidence } \\
\text { (www.cochranemsk.o } \\
\text { rg) that supervised } \\
\text { aerobic exercise } \\
\text { training has beneficial } \\
\text { effects on physical } \\
\text { capacity and FM } \\
\text { symptoms. Strength } \\
\text { training may also }\end{array}$ & \\
\hline
\end{tabular}




\begin{tabular}{|c|c|c|}
\hline & & $\begin{array}{l}\text { have benefits on some FM } \\
\text { symptoms. } \\
\text { Further studies on muscle } \\
\text { strengthening and flexibility } \\
\text { are needed. Research on the } \\
\text { long-term benefit of exercise } \\
\text { for FM is needed. - See more } \\
\text { at: } \\
\text { http://summaries.coch } \\
\text { rane.org/CD003786/ } \\
\text { MUSKEL_exercise- } \\
\text { forfibromyalgia\#sthash. } \\
\text { BzwierwV.dpuf }\end{array}$ \\
\hline $\begin{array}{l}\text { Resistance exercise training } \\
\text { for fibromyalgia;"Resistance } \\
\text { training (such as } \\
\text { weightlifting) for } \\
\text { fibromyalgia";CD010884;http } \\
\text { ://summaries.cochrane.org/C } \\
\text { D010884/MUSKEL_resistanc } \\
\text { e-training-such-as- } \\
\text { weightlifting-for-fibromyalgia }\end{array}$ & resistance training & $\begin{array}{l}\text { For women with } \\
\text { fibromyalgia, resistance } \\
\text { training for } 16 \text { to } 21 \text { weeks } \\
\text { likely improves } \\
-\quad \text { ability to do normal } \\
\text { activities } \\
-\quad \text { pain, tenderness, } \\
\text { muscle strength and overall } \\
\text { well-being. Quality of the } \\
\text { evidence -Because so few } \\
\text { studies have been done so far, } \\
\text { more research is likely to } \\
\text { change these results. } \\
-\quad \text { See more at: } \\
\text { http://summaries.coch } \\
\text { rane.org/CD010884/ } \\
\text { MUSKEL_resistancetraining- } \\
\text { such-asweight-lifting- } \\
\text { forfibromyalgia\#sthash. } \\
\text { 76OJ4uGl.dpuf }\end{array}$ \\
\hline
\end{tabular}




\begin{tabular}{|c|c|c|}
\hline $\begin{array}{l}\text { Acupuncture for treating } \\
\text { fibromyalgia;"Acupuncture } \\
\text { for } \\
\text { fibromyalgia";CD007070;http } \\
\text { ://summaries.cochrane.org/C } \\
\text { D007070/MUSKEL_acupunc } \\
\text { ture-for-fibromyalgia }\end{array}$ & $\begin{array}{l}\text { electric acupuncture } \\
\text { and manual } \\
\text { acupuncture }\end{array}$ & $\begin{array}{l}\text { There is low to } \\
\text { moderate-level } \\
\text { evidence that } \\
\text { compared with no } \\
\text { treatment and } \\
\text { standard therapy, } \\
\text { acupuncture improves } \\
\text { pain and stiffness in } \\
\text { people with } \\
\text { fibromyalgia. There is } \\
\text { moderate-level } \\
\text { evidence that the } \\
\text { effect of acupuncture } \\
\text { does not differ from } \\
\text { sham acupuncture in } \\
\text { reducing pain or } \\
\text { fatigue, or improving } \\
\text { sleep or global } \\
\text { wellbeing. EA is } \\
\text { probably better than } \\
\text { MA for pain and } \\
\text { stiffness reduction } \\
\text { and improvement of } \\
\text { global well-being, } \\
\text { sleep and fatigue. The } \\
\text { effect lasts up to one } \\
\text { month, but is not } \\
\text { maintained at six } \\
\text { months follow-up. } \\
\text { MA probably does } \\
\text { not improve pain or } \\
\text { physical functioning. } \\
\text { Acupuncture appears } \\
\text { safe. People with } \\
\text { fibromyalgia may } \\
\text { consider using EA } \\
\text { alone or with exercise } \\
\text { and medication. The } \\
\text { small sample size, } \\
\text { scarcity of studies for } \\
\text { each comparison, lack } \\
\text { of an ideal sham } \\
\text { acupuncture weaken } \\
\text { the level of evidence } \\
\text { and its clinical } \\
\text { implications. Larger } \\
\text { studies are warranted. } \\
\text { - See more at: } \\
\text { http://summaries.coch } \\
\text { rane.org/CD007070/ } \\
\text { MUSKEL_acupunctu }\end{array}$ \\
\hline
\end{tabular}




\begin{tabular}{|c|c|c|c|}
\hline & & $\begin{array}{l}\text { re- } \\
\text { forfibromyalgia\#sthash. } \\
\text { ZhGfzRsC.dpuf }\end{array}$ & \\
\hline $\begin{array}{l}\text { Cognitive behavioural } \\
\text { therapies for } \\
\text { fibromyalgia;"Cognitive } \\
\text { behavioural therapies for } \\
\text { fibromyalgia } \\
\text { syndrome";CD009796;http://s } \\
\text { ummaries.cochrane.org/CD00 } \\
\text { 9796/cognitive- } \\
\text { behaviouraltherapies-for- } \\
\text { fibromyalgiasyndrome }\end{array}$ & CBT & $\begin{array}{l}\text { CBTs provided a small } \\
\text { incremental benefit } \\
\text { over control } \\
\text { interventions in } \\
\text { reducing pain, negative } \\
\text { mood and disability at } \\
\text { the end of treatment } \\
\text { and at long-term } \\
\text { follow-up. }\end{array}$ & $\begin{array}{l}23 \text { studies with up } \\
\text { to } 2031 \text { people }\end{array}$ \\
\hline $\begin{array}{l}\text { Phenytoin for neuropathic } \\
\text { pain and fibromyalgia in } \\
\text { adults;"Phenytoin for } \\
\text { neuropathic pain and } \\
\text { fibromyalgia in } \\
\text { adults";CD009485;http://sum } \\
\text { maries.cochrane.org/CD0094 } \\
\text { 85/SYMPT_phenytoin- } \\
\text { forneuropathic-pain- } \\
\text { andfibromyalgia-in-adults }\end{array}$ & $\begin{array}{l}\text { phenytoin is for } \\
\text { neuropathic pain and } \\
\text { fibromyalgia. - See } \\
\text { more at: } \\
\text { http://summaries.coc } \\
\text { hrane.org/CD00948 } \\
\text { 5/SYMPT_phenytoi } \\
\text { n-for- } \\
\text { neuropathicpain- } \\
\text { andfibromyalgia- } \\
\text { inadults\#sthash.BniKq } \\
\text { Zkm.dpuf }\end{array}$ & $\begin{array}{l}\text { No studies met } \\
\text { inclusion criteria }\end{array}$ & \\
\hline $\begin{array}{l}\text { Multidisciplinary } \\
\text { rehabilitation for } \\
\text { fibromyalgia and } \\
\text { musculoskeletal pain in } \\
\text { working age } \\
\text { adults;"Multidisciplinary } \\
\text { rehabilitation for } \\
\text { fibromyalgia and } \\
\text { musculoskeletal pain in } \\
\text { working age } \\
\text { adults";CD001984;http://sum } \\
\text { maries.cochrane.org/CD0019 } \\
\text { 84/MUSKEL_multidisciplina }\end{array}$ & $\begin{array}{l}\text { the combo of } \\
\text { psychological, } \\
\text { behavioral and } \\
\text { educational } \\
\text { interventions } \\
\text { practiced in pain } \\
\text { clinics and } \\
\text { rehabilitation centers }\end{array}$ & $\begin{array}{l}\text { little scientific evidence } \\
\text { for the effectiveness of } \\
\text { multidisciplinary } \\
\text { rehabilitation for these } \\
\text { musculoskeletal } \\
\text { disorders }\end{array}$ & $\begin{array}{l}1808 \text { abstracts, and } \\
\text { the references of } 65 \\
\text { reviews, we found } \\
\text { only seven relevant } \\
\text { studies ( } 1050 \\
\text { patients) }\end{array}$ \\
\hline
\end{tabular}




\begin{tabular}{|c|c|c|c|}
\hline $\begin{array}{l}\text { ry-rehabilitation- } \\
\text { forfibromyalgia- } \\
\text { andmusculoskeletal-pain- } \\
\text { inworking-age-adults }\end{array}$ & & & \\
\hline $\begin{array}{l}\text { Clonazepam for neuropathic } \\
\text { pain and fibromyalgia in } \\
\text { adults;"Clonazepam for } \\
\text { neuropathic pain and } \\
\text { fibromyalgia in } \\
\text { adults";CD009486;http://sum } \\
\text { maries.cochrane.org/CD0094 } \\
\text { 86/SYMPT_clonazepam- } \\
\text { forneuropathic-pain- } \\
\text { andfibromyalgia-in-adults }\end{array}$ & $\begin{array}{l}\text { Some antiepileptic } \\
\text { medications can help } \\
\text { neuropathic pain. } \\
\text { Clonazepam is an } \\
\text { antiepileptic } \\
\text { medication }\end{array}$ & $\begin{array}{l}\text { no good quality } \\
\text { studies }\end{array}$ & \\
\hline $\begin{array}{l}\text { Gabapentin for chronic } \\
\text { neuropathic pain and } \\
\text { fibromyalgia in } \\
\text { adults;"Gabapentin for chronic } \\
\text { neuropathic pain and } \\
\text { fibromyalgia in } \\
\text { adults";CD007938;http://sum } \\
\text { maries.cochrane.org/CD0079 } \\
\text { 38/SYMPT_gabapentin- } \\
\text { forchronic-neuropathic- } \\
\text { painand-fibromyalgia-in- } \\
\text { adults }\end{array}$ & $\begin{array}{l}\text { Medicines like } \\
\text { paracetamol or } \\
\text { ibuprofen are not } \\
\text { effective in } \\
\text { neuropathic pain, } \\
\text { while medicines that } \\
\text { are sometimes used } \\
\text { to treat depression or } \\
\text { epilepsy can be very } \\
\text { effective in some } \\
\text { people with } \\
\text { neuropathic pain. } \\
\text { Our understanding of } \\
\text { fibromyalgia (a } \\
\text { condition of } \\
\text { persistent, } \\
\text { widespread pain and } \\
\text { tenderness, sleep } \\
\text { problems, and } \\
\text { fatigue) is poor, but } \\
\text { fibromyalgia can } \\
\text { respond to the same } \\
\text { medicines as } \\
\text { neuropathic pain. } \\
\text { Gabapentin was } \\
\text { developed to treat } \\
\text { epilepsy, but it is } \\
\text { now used to treat } \\
\text { various forms of } \\
\text { chronic pain. }\end{array}$ & $\begin{array}{l}\text { There were } \\
\text { insufficient data for } \\
\text { direct comparisons } \\
\text { with other active } \\
\text { treatments, and only } \\
\text { third tier evidence for } \\
\text { other painful } \\
\text { conditions. }\end{array}$ & $\begin{array}{l}\text { Seven new studies } \\
\text { with } 1919 \text { participants } \\
\text { were added in this } \\
\text { third update. }\end{array}$ \\
\hline
\end{tabular}




\begin{tabular}{|c|c|c|c|}
\hline $\begin{array}{l}\text { Milnacipran for neuropathic } \\
\text { pain and fibromyalgia in } \\
\text { adults;"Milnacipran for } \\
\text { chronic neuropathic pain and } \\
\text { fibromyalgia in } \\
\text { adults";CD008244;http://sum } \\
\text { maries.cochrane.org/CD0082 } \\
\text { 44/SYMPT_milnacipran- } \\
\text { forchronic-neuropathic- } \\
\text { painand-fibromyalgia-in- } \\
\text { adults }\end{array}$ & $\begin{array}{l}\text { milnacipran is for } \\
\text { treating chronic } \\
\text { neuropathic pain or } \\
\text { fibromyalgia. We } \\
\text { identified no studies } \\
\text { using milnacipran in } \\
\text { neuropathic pain, but } \\
\text { five studies in } \\
\text { fibromyalgia satisfied } \\
\text { the inclusion criteria. } \\
\text { - See more at: } \\
\text { http://summaries.coc } \\
\text { hrane.org/CD00824 } \\
\text { 4/SYMPT_milnacipr } \\
\text { an-for- } \\
\text { chronicneuropathic- } \\
\text { painand-fibromyalgia- } \\
\text { inadults\#sthash.sszfV2 } \\
\text { Ug.dpuf }\end{array}$ & $\begin{array}{l}\text { Milnacipran has shown } \\
\text { modest effects in a } \\
\text { minority of participants } \\
\text { with fibromyalgia, and } \\
\text { several technical issues } \\
\text { indicate that even this } \\
\text { modest effect may } \\
\text { overstate effectiveness } \\
\text { in clinical practice The } \\
\text { drug may be a useful } \\
\text { option if first-line } \\
\text { treatments fail. - See } \\
\text { more at: } \\
\text { http://summaries.coch } \\
\text { rane.org/CD008244/S } \\
\text { YMPT_milnacipranfor- } \\
\text { chronicneuropathic- } \\
\text { pain-andfibromyalgia- } \\
\text { inadults\#sthash.sszfV2 } \\
\text { Ug.dpuf }\end{array}$ & $\begin{array}{l}4000 \text { participants } \\
\text { treated with } \\
\text { milnacipran } 100 \mathrm{mg} \text { or } \\
200 \mathrm{mg} \text {, or placebo, for } \\
\text { eight to } 24 \text { weeks at the } \\
\text { target dose. - See more } \\
\text { at: } \\
\text { http://summaries.coch } \\
\text { rane.org/CD008244/S } \\
\text { YMPT_milnacipranfor- } \\
\text { chronicneuropathic- } \\
\text { pain-andfibromyalgia- } \\
\text { inadults\#sthash.sszfV2 } \\
\text { Ug.dpuf }\end{array}$ \\
\hline $\begin{array}{l}\text { Amitriptyline for neuropathic } \\
\text { pain and fibromyalgia in } \\
\text { adults;"Amitriptyline for } \\
\text { neuropathic pain and } \\
\text { fibromyalgia in } \\
\text { adults";CD008242;http://sum } \\
\text { maries.cochrane.org/CD0082 } \\
\text { 42/SYMPT_amitriptyline- } \\
\text { forneuropathic-pain- } \\
\text { andfibromyalgia-in-adults }\end{array}$ & $\begin{array}{l}\text { Amitriptyline is a } \\
\text { tricyclic } \\
\text { antidepressant that is } \\
\text { widely used to treat } \\
\text { chronic neuropathic } \\
\text { pain (pain due to } \\
\text { nerve damage) and } \\
\text { fibromyalgia, and is } \\
\text { recommended in many } \\
\text { guidelines. }\end{array}$ & $\begin{array}{l}\text { There was no top-tier } \\
\text { evidence for } \\
\text { amitriptyline in treating } \\
\text { neuropathic pain or } \\
\text { fibromyalgia. Second- } \\
\text { tier evidence indicated } \\
\text { some evidence of effect } \\
\text { in painful diabetic } \\
\text { neuropathy (PDN), } \\
\text { mixed neuropathic } \\
\text { pain, and fibromyalgia. } \\
\text { Amitriptyline has been } \\
\text { a first-line treatment } \\
\text { for neuropathic pain for } \\
\text { many years. The fact } \\
\text { that there is no } \\
\text { supportive unbiased } \\
\text { evidence for a } \\
\text { beneficial effect is } \\
\text { disappointing, but has } \\
\text { to be balanced against } \\
\text { decades of successful } \\
\text { treatment in many } \\
\text { patients with } \\
\text { neuropathic pain or }\end{array}$ & $\begin{array}{l}\text { Twenty-one studies } \\
\text { (1437 participants) } \\
\text { were included; they } \\
\text { individually involved } \\
\text { between } 15 \text { and } 235 \\
\text { participants, only four } \\
\text { involved over } 100 \\
\text { participants, and the } \\
\text { median study size was } \\
44 \text { participants. The } \\
\text { median duration was } \\
\text { six weeks. Ten studies } \\
\text { had a crossover design. } \\
\text { Doses of amitriptyline } \\
\text { were generally } \\
\text { between } 25 \text { mg and } \\
125 \text { mg, and dose } \\
\text { escalation was } \\
\text { common. }\end{array}$ \\
\hline
\end{tabular}




\begin{tabular}{|c|c|c|c|}
\hline & & $\begin{array}{l}\text { fibromyalgia. There is } \\
\text { no good evidence of a } \\
\text { lack of effect; rather } \\
\text { our concern should be } \\
\text { of overestimation of } \\
\text { treatment effect. } \\
\text { Amitriptyline should } \\
\text { continue to be used as } \\
\text { part of the treatment } \\
\text { of neuropathic pain or } \\
\text { fibromyalgia, but } \\
\text { only a minority of } \\
\text { patients will achieve } \\
\text { satisfactory pain } \\
\text { relief. }\end{array}$ & \\
\hline $\begin{array}{l}\text { Duloxetine for treating painful } \\
\text { neuropathy, chronic pain or } \\
\text { fibromyalgia;"Duloxetine for } \\
\text { treating chronic pain or } \\
\text { fibromyalgia";CD007115;http } \\
\text { ://summaries.cochrane.org/C } \\
\text { D007115/NEUROMUSC_dul } \\
\text { oxetine-for-treating- } \\
\text { painfulneuropathy-chronic- } \\
\text { pain-orfibromyalgia painful } \\
\text { neuropathy }\end{array}$ & $\begin{array}{l}\text { Duloxetine is a } \\
\text { balanced serotonin } \\
\text { and noradrenaline } \\
\text { reuptake inhibitor } \\
\text { licensed for the } \\
\text { treatment of major } \\
\text { depressive disorders, } \\
\text { urinary stress } \\
\text { incontinence and the } \\
\text { management of } \\
\text { neuropathic pain } \\
\text { associated with } \\
\text { diabetic peripheral } \\
\text { neuropathy. }\end{array}$ & $\begin{array}{l}\text { There are adequate } \\
\text { amounts of moderate } \\
\text { quality evidence from } \\
\text { eight studies } \\
\text { performed by the } \\
\text { manufacturers of } \\
\text { duloxetine that doses } \\
\text { of } 60 \text { mg and } 120 \mathrm{mg} \\
\text { daily are efficacious } \\
\text { for treating pain in } \\
\text { diabetic peripheral } \\
\text { neuropathy but lower } \\
\text { daily doses are not. } \\
\text { Further trials are not } \\
\text { required. In } \\
\text { fibromyalgia, there is } \\
\text { lower quality } \\
\text { evidence that } \\
\text { duloxetine is effective } \\
\text { at similar doses to } \\
\text { those used in diabetic } \\
\text { peripheral neuropathy } \\
\text { and with a similar } \\
\text { magnitude of effect. } \\
\text { The effect in } \\
\text { fibromyalgia may be } \\
\text { achieved through a } \\
\text { greater improvement } \\
\text { in mental symptoms } \\
\text { than in somatic } \\
\text { physical pain. There is } \\
\text { low to moderate } \\
\text { quality evidence that } \\
\text { pain relief is also } \\
\text { achieved in pain }\end{array}$ & $\begin{array}{l}18 \text { trials, which } \\
\text { included } 6407 \\
\text { participants. We } \\
\text { found } 12 \text { of these } \\
\text { studies in the } \\
\text { literature search for } \\
\text { this update. Eight } \\
\text { studies included a } \\
\text { total of } 2728 \\
\text { participants with } \\
\text { painful diabetic } \\
\text { neuropathy and six } \\
\text { studies involved } 2249 \\
\text { participants with } \\
\text { fibromyalgia. Three } \\
\text { studies included } \\
\text { participants with } \\
\text { depression and painful } \\
\text { physical symptoms } \\
\text { and one included } \\
\text { participants with } \\
\text { central neuropathic } \\
\text { pain. }\end{array}$ \\
\hline
\end{tabular}




\begin{tabular}{|c|c|c|c|}
\hline & & $\begin{array}{l}\text { associated with } \\
\text { depressive symptoms, } \\
\text { but the NNTB of } 8 \text { in } \\
\text { fibromyalgia and } \\
\text { depression is not an } \\
\text { indication of } \\
\text { substantial efficacy. }\end{array}$ & \\
\hline $\begin{array}{l}\text { Lacosamide for neuropathic } \\
\text { pain and fibromyalgia in } \\
\text { adults;"Lacosamide for } \\
\text { neuropathic pain and } \\
\text { fibromyalgia in } \\
\text { adults";CD009318;http://sum } \\
\text { maries.cochrane.org/CD0093 } \\
\text { 18/NEUROMUSC_lacosamid } \\
\text { e-for-neuropathic-pain- } \\
\text { andfibromyalgia-in-adults }\end{array}$ & $\begin{array}{l}\text { Antiepileptic drugs } \\
\text { like lacosamide }\end{array}$ & $\begin{array}{l}\text { In people with painful } \\
\text { diabetic neuropathy, } \\
\text { lacosamide had only a } \\
\text { modest effect, with a } \\
\text { specific effect due to } \\
\text { its use in } 1 \text { person in } \\
\text { 10. This is a minor } \\
\text { effect and may be an } \\
\text { over-estimate due to } \\
\text { use of the last } \\
\text { observation carried } \\
\text { forward method for } \\
\text { analysis. There was } \\
\text { insufficient } \\
\text { information in } \\
\text { fibromyalgia to draw } \\
\text { any conclusions about } \\
\text { the effect of } \\
\text { lacosamide. - See more } \\
\text { at: } \\
\text { http://summaries.coch } \\
\text { rane.org/CD009318/ } \\
\text { NEUROMUSC_lacos } \\
\text { amide-for- } \\
\text { neuropathic-pain- } \\
\text { andfibromyalgia- } \\
\text { inadults\#sthash.6W9bli } \\
\text { Q5.dpuf }\end{array}$ & $\begin{array}{l}\text { five studies in painful } \\
\text { diabetic neuropathy } \\
\text { (1863 participants) and } \\
\text { one in fibromyalgia } \\
\text { (159 participants). - } \\
\text { See more at: } \\
\text { http://summaries.coch } \\
\text { rane.org/CD009318/ } \\
\text { NEUROMUSC_lacos } \\
\text { amide-forneuropathic- } \\
\text { pain-andfibromyalgia- } \\
\text { inadults\#sthash.6W9bIi } \\
\text { Q5.dpuf }\end{array}$ \\
\hline
\end{tabular}




\begin{tabular}{|c|c|c|c|}
\hline $\begin{array}{l}\text { Topiramate for neuropathic pain } \\
\text { and fibromyalgia in } \\
\text { adults;"Topiramate for treating } \\
\text { neuropathic pain or } \\
\text { fibromyalgia";CD008314;http } \\
\text { ://summaries.cochrane.org/C } \\
\text { D008314/NEUROMUSC_top } \\
\text { iramate-for-treatingneuropathic- } \\
\text { pain-orfibromyalgia }\end{array}$ & $\begin{array}{l}\text { Topiramate is a } \\
\text { medicine used to } \\
\text { treat epilepsy, } \\
\text { and so it might } \\
\text { be a useful } \\
\text { medicine for } \\
\text { neuropathic pain } \\
\text { or fibromyalgia. }\end{array}$ & $\begin{array}{l}\text { Topiramate did not help } \\
\text { the pain and was no } \\
\text { different from placebo } \\
\text { except in causing more } \\
\text { sideeffects, which made } \\
\text { many more people } \\
\text { withdraw from the } \\
\text { studies early. About } 3 \\
\text { people in } 10 \text { withdrew } \\
\text { because of side-effects } \\
\text { with topiramate } \\
\text { compared with } 1 \text { in } 10 \\
\text { with placebo. } \\
\text { Topiramate has not been } \\
\text { shown to work as a pain } \\
\text { medicine in diabetic } \\
\text { neuropathy. }\end{array}$ & $\begin{array}{l}\text { four studies of } \\
\text { reasonable quality that } \\
\text { tested topiramate } \\
\text { against placebo for a } \\
\text { number of weeks. } \\
\text { Almost all of the } 1684 \\
\text { people in the studies } \\
\text { had painful limbs } \\
\text { because of damaged } \\
\text { nerves caused by } \\
\text { diabetes. - See more } \\
\text { at: } \\
\text { http://summaries.coch } \\
\text { rane.org/CD008314/ } \\
\text { NEUROMUSC_topir } \\
\text { amate-for- } \\
\text { treatingneuropathic- } \\
\text { pain- } \\
\text { orfibromyalgia\#sthash. } \\
\text { 9txXjI9A.dpuf }\end{array}$ \\
\hline $\begin{array}{l}\text { Monoamine oxidase inhibitors } \\
\text { (MAOIs) for fibromyalgia } \\
\text { syndrome;"Monoamine oxidase } \\
\text { inhibitors (MAOIs) for } \\
\text { fibromyalgia";CD009807;http } \\
\text { ://summaries.cochrane.org/C } \\
\text { D009807/monoamine- } \\
\text { oxidase-inhibitors-maois- } \\
\text { forfibromyalgia }\end{array}$ & $\begin{array}{l}\text { MAOIs may } \\
\text { slightly improve } \\
\text { pain and tender } \\
\text { points in the } \\
\text { short term } \\
\text { compared to } \\
\text { placebo. Of the } \\
\text { MAOIs studied, } \\
\text { pirlindole seems } \\
\text { more effective } \\
\text { than } \\
\text { moclobemide. }\end{array}$ & $\begin{array}{l}\text { Pirlindole showed } \\
\text { statistically } \\
\text { significant results } \\
\text { compared with placebo } \\
\text { for several outcomes } \\
\text { (pain, tender points and } \\
\text { overall assessment by } \\
\text { the patient and the } \\
\text { physician), whereas } \\
\text { moclobemide did not } \\
\text { show statistically } \\
\text { significant differences } \\
\text { between groups. }\end{array}$ & $\begin{array}{l}\text { two studies of } \\
\text { inconsistent risk of } \\
\text { bias with a total of } 230 \\
\text { patients diagnosed } \\
\text { with FM. We } \\
\text { evaluated two MAOIs: } \\
\text { pirlindole and } \\
\text { moclobemide. }\end{array}$ \\
\hline $\begin{array}{l}\text { Valproic acid and sodium } \\
\text { valproate for neuropathic pain } \\
\text { and fibromyalgia in } \\
\text { adults;"Valproic acid and } \\
\text { sodium valproate for } \\
\text { neuropathic pain and } \\
\text { fibromyalgia";CD009183;http } \\
\text { ://summaries.cochrane.org/C } \\
\text { D009183/SYMPT_valproicacid- } \\
\text { and-sodium-valproatefor- } \\
\text { neuropathic-pain- } \\
\text { andfibromyalgia }\end{array}$ & $\begin{array}{l}\text { Valproic acid } \\
\text { and its sodium } \\
\text { salt (sodium } \\
\text { valproate) are } \\
\text { antiepileptic } \\
\text { drugs that are } \\
\text { sometimes used } \\
\text { to treat chronic } \\
\text { neuropathic pain } \\
\text { and } \\
\text { fibromyalgia, } \\
\text { although they } \\
\text { are not licensed } \\
\text { for this use. }\end{array}$ & $\begin{array}{l}\text { There is insufficient } \\
\text { evidence to support the } \\
\text { use of valproic acid or } \\
\text { sodium valproate as a } \\
\text { firstline treatment for } \\
\text { neuropathic pain. - See } \\
\text { more at: } \\
\text { http://summaries.coch } \\
\text { rane.org/CD009183/S } \\
\text { YMPT_valproic- } \\
\text { acidand- } \\
\text { sodiumvalproate- } \\
\text { forneuropathic-pain- } \\
\text { andfibromyalgia\#sthash. } \\
\text { xiAvcyI1.dpuf }\end{array}$ & $\begin{array}{l}\text { three studies, two in } \\
\text { diabetic neuropathy } \\
\text { ( } 42 \text { participants } \\
\text { treated with valproate, } \\
42 \text { with placebo), and } \\
\text { one in post-herpetic } \\
\text { neuralgia ( } 23 \text { treated } \\
\text { with divalproex } \\
\text { sodium, } 22 \text { with } \\
\text { placebo). Study } \\
\text { duration was eight or } \\
12 \text { weeks. No studies } \\
\text { were found in } \\
\text { fibromyalgia. }\end{array}$ \\
\hline
\end{tabular}




\begin{tabular}{|c|c|c|c|}
\hline $\begin{array}{l}\text { Oxycodone for neuropathic } \\
\text { pain and fibromyalgia in } \\
\text { adults;"Oxycodone for } \\
\text { neuropathic pain and } \\
\text { fibromyalgia in } \\
\text { adults";CD010692;http://sum } \\
\text { maries.cochrane.org/CD0106 } \\
\text { 92/SYMPT_oxycodone- } \\
\text { forneuropathic-pain- } \\
\text { andfibromyalgia-in-adults }\end{array}$ & $\begin{array}{l}\text { Oxycodone is used to } \\
\text { treat acute and some } \\
\text { forms of chronic pain, } \\
\text { and so might be a } \\
\text { useful medicine for } \\
\text { neuropathic pain or } \\
\text { fibromyalgia. - See } \\
\text { more at: } \\
\text { http://summaries.coc } \\
\text { hrane.org/CD01069 } \\
\text { 2/SYMPT_oxycodo } \\
\text { ne-for- } \\
\text { neuropathicpain- } \\
\text { andfibromyalgia- } \\
\text { inadults\#sthash.VtfS51 } \\
\text { 3z.dpuf }\end{array}$ & $\begin{array}{l}\text { Oxycodone was not } \\
\text { convincingly shown } \\
\text { to help relieve the } \\
\text { pain (very low quality } \\
\text { evidence). Compared } \\
\text { with placebo, fewer } \\
\text { people stopped taking } \\
\text { oxycodone because } \\
\text { they felt it was not } \\
\text { effective, but more } \\
\text { people experienced } \\
\text { adverse effects (very } \\
\text { low quality } \\
\text { evidence). } \\
\text { Oxycodone has not } \\
\text { been shown to work } \\
\text { as a pain medicine in } \\
\text { diabetic neuropathy } \\
\text { or postherpetic } \\
\text { neuralgia. }\end{array}$ & $\begin{array}{l}\text { three studies of } \\
\text { modest quality that } \\
\text { tested oxycodone } \\
\text { against placebo for } \\
\text { several weeks. } \\
\text { Almost all of the } 254 \\
\text { people in the studies } \\
\text { had painful limbs } \\
\text { because of damaged } \\
\text { nerves caused by } \\
\text { diabetes. No studies } \\
\text { have examined its use } \\
\text { in other types of } \\
\text { neuropathic pain, or } \\
\text { in fibromyalgia. }\end{array}$ \\
\hline
\end{tabular}




\begin{tabular}{|c|c|c|}
\hline $\begin{array}{l}\text { Antiepileptic drugs for } \\
\text { neuropathic pain and } \\
\text { fibromyalgia - an overview of } \\
\text { Cochrane } \\
\text { reviews;"Antiepileptic drugs to } \\
\text { treat neuropathic pain or } \\
\text { fibromyalgia- an overview of } \\
\text { Cochrane } \\
\text { reviews";CD010567;http://su } \\
\text { mmaries.cochrane.org/CD010 } \\
\text { 567/SYMPT_antiepilepticdrugs- } \\
\text { to-treat-neuropathicpain-or- } \\
\text { fibromyalgia-anoverview-of- } \\
\text { cochranereviews }\end{array}$ & $\begin{array}{l}\text { Antiepileptic drugs } \\
\text { (previously called } \\
\text { anticonvulsants) are } \\
\text { used for treating } \\
\text { epilepsy, but have } \\
\text { also been used for } \\
\text { treating neuropathic } \\
\text { pain and } \\
\text { fibromyalgia. Many } \\
\text { of the drugs have } \\
\text { been the subject of } \\
\text { individual Cochrane } \\
\text { reviews. In August } \\
\text { 2013 we collected } \\
\text { all these Cochrane } \\
\text { reviews on } \\
\text { antiepileptic drugs } \\
\text { together to provide } \\
\text { an overview. } \\
\text { Individual } \\
\text { antiepileptic drugs } \\
\text { work in different } \\
\text { ways, and there is no } \\
\text { expectation that they } \\
\text { are equally effective. } \\
\text { - See more at: } \\
\text { http://summaries.coc } \\
\text { hrane.org/CD01056 } \\
7 / S Y M P T \text { antiepile } \\
\text { ptic-drugs-to- } \\
\text { treatneuropathic- } \\
\text { pain-orfibromyalgia- } \\
\text { anoverview- } \\
\text { ofcochrane- } \\
\text { reviews\#sthash.xqm } \\
\text { OPsy4.dpuf }\end{array}$ & $\begin{array}{l}\text { No studies reported } \\
\text { top tier results. We } \\
\text { found that only for } \\
\text { gabapentin and } \\
\text { pregabalin was there } \\
\text { some evidence that } \\
\text { they worked in } \\
\text { longterm nerve pain } \\
\text { with diabetes (painful } \\
\text { diabetic neuropathy) } \\
\text { and pain after } \\
\text { shingles (postherpetic } \\
\text { neuralgia). } \\
\text { Pregabalin also had } \\
\text { evidence of efficacy } \\
\text { in central neuropathic } \\
\text { pain (typically pain } \\
\text { after stroke) and in } \\
\text { fibromyalgia. The } \\
\text { drugs work very well } \\
\text { in some people with } \\
\text { these painful } \\
\text { conditions, with pain } \\
\text { reduced by half. } \\
\text { However, only } \\
\text { between } 1 \text { in } 10 \text { and } 1 \\
\text { in } 4 \text { people will get } \\
\text { this level of benefit, } \\
\text { depending on the } \\
\text { pain condition and } \\
\text { the drug. Most people } \\
\text { will get no pain } \\
\text { relief. } \\
\text { The antiepileptic } \\
\text { drugs produced side } \\
\text { effects in most } \\
\text { people taking them, } \\
\text { and for about } 1 \text { in } 4 \\
\text { these could not be } \\
\text { tolerated so they } \\
\text { stopped taking the } \\
\text { drug. Serious side } \\
\text { effects were no more } \\
\text { common with } \\
\text { antiepileptic drugs } \\
\text { than with a harmless } \\
\text { placebo. } \\
\text { found did not meet } \\
\text { current best }\end{array}$ \\
\hline
\end{tabular}




\begin{tabular}{|c|c|c|c|}
\hline & & $\begin{array}{l}\text { standards, and as a } \\
\text { result it may } \\
\text { overestimate benefit. } \\
\text { The biggest concern is } \\
\text { a lack of any evidence } \\
\text { for most drugs in } \\
\text { most types of } \\
\text { neuropathic pain and } \\
\text { fibromyalgia. For } \\
\text { lacosamide and } \\
\text { lamotrigine there is } \\
\text { evidence of a lack of } \\
\text { effect; for other } \\
\text { antiepileptic drugs } \\
\text { (including } \\
\text { carbamazepine, } \\
\text { clonazepam, } \\
\text { phenytoin, valproate) } \\
\text { there is no evidence of } \\
\text { effect or insufficient } \\
\text { evidence of effect. }\end{array}$ & \\
\hline $\begin{array}{l}\text { Serotonin and noradrenaline } \\
\text { reuptake inhibitors (SNRIs) } \\
\text { for fibromyalgia } \\
\text { syndrome;"Serotonin and } \\
\text { noradrenaline reuptake } \\
\text { inhibitors for } \\
\text { fibromyalgia";CD010292;http } \\
\text { ://summaries.cochrane.org/C } \\
\text { D010292/serotonin- } \\
\text { andnoradrenaline- } \\
\text { reuptakeinhibitors-for- } \\
\text { fibromyalgia }\end{array}$ & $\begin{array}{l}\text { serotonin and } \\
\text { noradrenaline } \\
\text { reuptake inhibitors } \\
\text { (SNRIs) }\end{array}$ & $\begin{array}{l}\text { The SNRIs duloxetine } \\
\text { and milnacipran } \\
\text { provided a small } \\
\text { incremental benefit } \\
\text { over placebo in } \\
\text { reducing pain. The } \\
\text { superiority of } \\
\text { duloxetine and } \\
\text { milnacipran over } \\
\text { placebo in reducing } \\
\text { fatigue and limitations } \\
\text { of QOL was not } \\
\text { substantial. } \\
\text { Duloxetine and } \\
\text { milnacipran were not } \\
\text { superior to placebo in } \\
\text { reducing sleep } \\
\text { problems. The } \\
\text { dropout rates due to }\end{array}$ & $\begin{array}{l}\text { Ten studies were } \\
\text { included with a total } \\
\text { of } 6038 \text { participants. } \\
\text { Five studies } \\
\text { investigated } \\
\text { duloxetine against } \\
\text { placebo, and five } \\
\text { investigated } \\
\text { milnacipran against } \\
\text { placebo. A total of } \\
3611 \text { participants were } \\
\text { included into } \\
\text { duloxetine or } \\
\text { milnacipran groups } \\
\text { and } 2427 \text { participants } \\
\text { into placebo groups. } \\
\text { The studies had a low } \\
\text { risk of bias in general. }\end{array}$ \\
\hline
\end{tabular}




\begin{tabular}{|c|c|c|c|}
\hline & & $\begin{array}{l}\text { adverse events were } \\
\text { higher for duloxetine } \\
\text { and milnacipran than } \\
\text { for placebo. }\end{array}$ & \\
\hline $\begin{array}{l}\text { Lamotrigine for chronic } \\
\text { neuropathic pain and } \\
\text { fibromyalgia in } \\
\text { adults;"Lamotrigine (an } \\
\text { antiepileptic drug) for chronic } \\
\text { neuropathic pain or } \\
\text { fibromyalgia";CD006044;http } \\
\text { ://summaries.cochrane.org/C } \\
\text { D006044/SYMPT_lamotrigin } \\
\text { e-an-antiepileptic-drug- } \\
\text { forchronic-neuropathic-pain- } \\
\text { orfibromyalgia }\end{array}$ & $\begin{array}{l}\text { Lamotrigine is a } \\
\text { medicine used to } \\
\text { treat epilepsy, and so } \\
\text { might be a useful } \\
\text { medicine for } \\
\text { neuropathic pain or } \\
\text { fibromyalgia. }\end{array}$ & $\begin{array}{l}\text { Lamotrigine did not } \\
\text { help the pain, and } \\
\text { was no different from } \\
\text { placebo except in } \\
\text { causing more side } \\
\text { effects. Adverse } \\
\text { events were more } \\
\text { frequent with } \\
\text { lamotrigine than } \\
\text { placebo, with rash in } \\
1 \text { person in } 27 . \text { - }\end{array}$ & $\begin{array}{l}12 \text { studies of } \\
\text { reasonable quality } \\
\text { that tested lamotrigine } \\
\text { against placebo for a } \\
\text { number of weeks. } \\
\text { Almost half of the } \\
1511 \text { people in the } \\
\text { studies had painful } \\
\text { limbs because of } \\
\text { damaged nerves } \\
\text { caused by diabetes, } \\
\text { and seven different } \\
\text { painful neuropathic } \\
\text { conditions were } \\
\text { examined. No studies } \\
\text { looked at } \\
\text { fibromyalgia. - See } \\
\text { more at: } \\
\text { http://summaries.coch } \\
\text { rane.org/CD006044/S } \\
\text { YMPT_lamotrigine- } \\
\text { an-antiepileptic- } \\
\text { drugfor- } \\
\text { chronicneuropathic- } \\
\text { pain- } \\
\text { orfibromyalgia\#sthash. } \\
\text { VyD9Kv3y.dpuf }\end{array}$ \\
\hline
\end{tabular}




\begin{tabular}{|c|c|c|c|}
\hline $\begin{array}{l}\text { Carbamazepine for chronic } \\
\text { neuropathic pain and } \\
\text { fibromyalgia in } \\
\text { adults;"Carbamazepine for } \\
\text { chronic neuropathic pain and } \\
\text { fibromyalgia in } \\
\text { adults";CD005451;http://sum } \\
\text { maries.cochrane.org/CD0054 } \\
\text { 51/SYMPT_carbamazepine- } \\
\text { for-chronic-neuropathic- } \\
\text { painand-fibromyalgia-in- } \\
\text { adults }\end{array}$ & $\begin{array}{l}\text { Carbamazepine was } \\
\text { developed to treat } \\
\text { epilepsy, but it is now } \\
\text { used to treat various } \\
\text { forms of chronic pain. } \\
\text { - See more at: } \\
\text { http://summaries.coc } \\
\text { hrane.org/CD00545 } \\
\text { 1/SYMPT_carbamaz } \\
\text { epine-for- } \\
\text { chronicneuropathic- } \\
\text { painand- } \\
\text { fibromyalgia- } \\
\text { inadults\#sthash.vNNP } \\
\text { XVgz.dpuf }\end{array}$ & $\begin{array}{l}\text { There was not } \\
\text { enough good quality } \\
\text { evidence to say how } \\
\text { well carbamazepine } \\
\text { worked in any } \\
\text { neuropathic pain } \\
\text { condition. Pooling } \\
\text { four small studies } \\
\text { showed that it was } \\
\text { better than placebo, } \\
\text { but the result cannot } \\
\text { be relied upon. } \\
\text { There was not } \\
\text { enough information } \\
\text { from these studies to } \\
\text { make any reliable } \\
\text { comment on adverse } \\
\text { events or harm. } \\
\text { Carbamazepine is } \\
\text { probably helpful for } \\
\text { some people with } \\
\text { chronic neuropathic } \\
\text { pain. It is not } \\
\text { possible to know } \\
\text { beforehand who will } \\
\text { benefit and who will } \\
\text { not. }\end{array}$ & $\begin{array}{l}10 \text { studies involving } \\
418 \text { people involved } \\
\text { in testing } \\
\text { carbamazepine. Studies } \\
\text { were not generally of } \\
\text { very good quality. Most } \\
\text { were very small, as } \\
\text { well as of short } \\
\text { duration. Studies } \\
\text { lasting only one or two } \\
\text { weeks are unhelpful } \\
\text { when pain can last for } \\
\text { years. - See more at: } \\
\text { http://summaries.coch } \\
\text { rane.org/CD005451/S } \\
\text { YMPT_carbamazepin } \\
\text { e-for- } \\
\text { chronicneuropathic- } \\
\text { pain-andfibromyalgia- } \\
\text { inadults\#sthash.vNNPX } \\
\text { Vgz.dpuf }\end{array}$ \\
\hline
\end{tabular}




\begin{tabular}{|c|c|c|c|}
\hline $\begin{array}{l}\text { PycnogenolÂ® (extract of } \\
\text { French maritime pine bark) } \\
\text { for the treatment of chronic } \\
\text { disorders;"Use of the } \\
\text { antioxidant supplement } \\
\text { PycnogenolÂß to treat a } \\
\text { variety of chronic } \\
\text { disorders";CD008294;http://s } \\
\text { ummaries.cochrane.org/CD00 } \\
\text { 8294/CF_use-of- } \\
\text { theantioxidant- } \\
\text { supplementpycnogenolr-to- } \\
\text { treat-avariety-of-chronic- } \\
\text { disorders }\end{array}$ & $\begin{array}{l}\text { Pycnogenol® is a } \\
\text { herbal dietary } \\
\text { supplement extracted } \\
\text { from French } \\
\text { maritime pine bark } \\
\text { whose main } \\
\text { ingredient is } \\
\text { procyanidin. } \\
\text { Procyanidin is a } \\
\text { powerful antioxidant } \\
\text { also found in food } \\
\text { such as grapes, } \\
\text { berries, } \\
\text { pomegranates, red } \\
\text { wine and various } \\
\text { nuts. Supplements } \\
\text { containing } \\
\text { procyanidin are } \\
\text { widely marketed } \\
\text { worldwide for the } \\
\text { promotion of health, } \\
\text { and for the } \\
\text { prevention and } \\
\text { treatment of chronic } \\
\text { disorders. The } \\
\text { rationale for this is } \\
\text { that antioxidants } \\
\text { neutralise reactive } \\
\text { oxygen species } \\
\text { (ROS; frequently } \\
\text { referred to as "free } \\
\text { radicals") which, } \\
\text { apart from } \\
\text { performing } \\
\text { important bodily } \\
\text { functions, can cause } \\
\text { damage to cells and } \\
\text { tissues if present in } \\
\text { excessive amounts. } \\
\text { Available } \\
\text { supplements vary in } \\
\text { the source and } \\
\text { quantity of } \\
\text { procyanidin, as well } \\
\text { as in number and } \\
\text { type of other } \\
\text { ingredients they } \\
\text { contain. We chose to } \\
\text { focus on } \\
\text { syent is a }\end{array}$ & $\begin{array}{l}\text { Due to small sample } \\
\text { size, limited number } \\
\text { of trials per condition, } \\
\text { variation in outcomes } \\
\text { evaluated and } \\
\text { outcome measures } \\
\text { used, as well as the } \\
\text { risk of bias in the } \\
\text { included studies, no } \\
\text { definite conclusions } \\
\text { regarding the efficacy } \\
\text { and safety of } \\
\text { Pycnogenol® are } \\
\text { possible. }\end{array}$ & $\begin{array}{l}15 \text { eligible } \\
\text { randomised controlled } \\
\text { trials with a total of } \\
791 \text { participants } \\
\text { which addressed } \\
\text { seven different } \\
\text { chronic conditions: } \\
\text { asthma (two studies); } \\
\text { attention deficit } \\
\text { hyperactivity disorder } \\
\text { (one study), chronic } \\
\text { venous insufficiency } \\
\text { (two studies), diabetes } \\
\text { (four studies), erectile } \\
\text { dysfunction (one } \\
\text { study), hypertension } \\
\text { (two studies) and } \\
\text { osteoarthritis (three } \\
\text { studies). }\end{array}$ \\
\hline
\end{tabular}




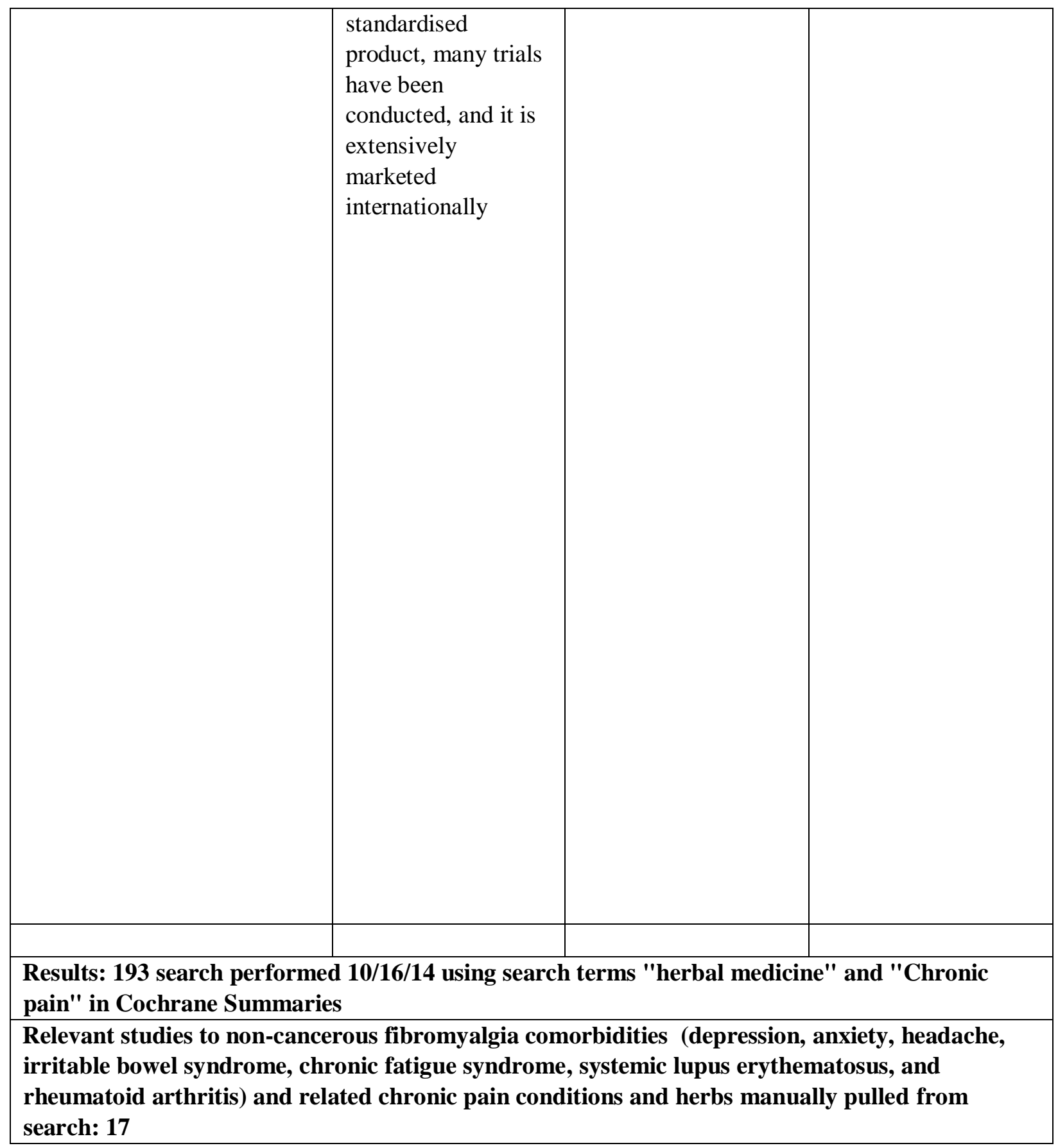




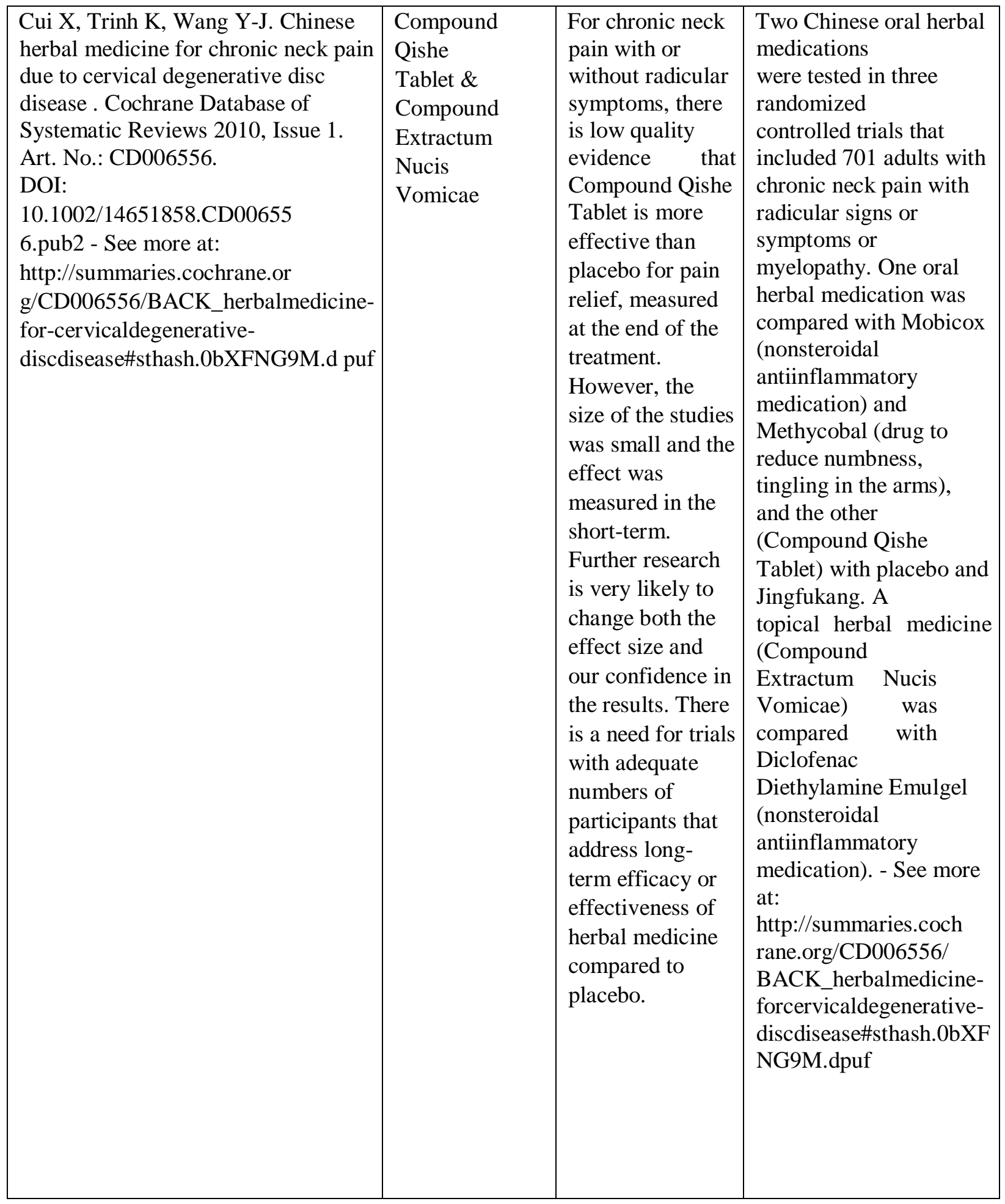




\begin{tabular}{|c|c|c|c|}
\hline $\begin{array}{l}\text { Gagnier JJ, van Tulder MW, Berman } \\
\text { BM, Bombardier C. Herbal medicine } \\
\text { for low back pain. Cochrane } \\
\text { Database of Systematic Reviews } \\
\text { 2006, Issue 2. Art. No.: CD004504. } \\
\text { DOI: } \\
\text { 10.1002/14651858.CD00450 4.pub3 } \\
\text { Assessed as up to date: } 15 \\
\text { December } 2005 \text { - See } \\
\text { more at: } \\
\text { http://summaries.cochrane.or } \\
\text { g/CD004504/BACK_herbalmedicine- } \\
\text { for-low- } \\
\text { backpain\#sthash.MDoTN5GZ.dpu f }\end{array}$ & $\begin{array}{l}\text { herbal medicine } \\
\text { for non-specific } \\
\text { lowback pain. }\end{array}$ & $\begin{array}{l}\text { Harpagophytum } \\
\text { Procumbens, Salix } \\
\text { Alba and Capsicum } \\
\text { Frutescens seem to } \\
\text { reduce pain more } \\
\text { than placebo. } \\
\text { Additional trials } \\
\text { testing these herbal } \\
\text { medicines against } \\
\text { standard treatments } \\
\text { are needed. The } \\
\text { quality of reporting } \\
\text { in these trials was } \\
\text { generally poor. Two } \\
\text { high quality trials } \\
\text { examining the } \\
\text { effects of } \\
\text { Harpagophytum } \\
\text { Procumbens } \\
\text { (Devil's Claw) } \\
\text { found strong } \\
\text { evidence that daily } \\
\text { doses standardized } \\
\text { to } 50 \text { mg or } 100 \text { mg } \\
\text { harpagoside were } \\
\text { better than placebo } \\
\text { for short-term } \\
\text { improvements in } \\
\text { pain and rescue } \\
\text { medication. } \\
\text { Another high } \\
\text { quality trial } \\
\text { demonstrated } \\
\text { relative equivalence } \\
\text { to } 12.5 \text { mg per day } \\
\text { of rofecoxib } \\
\text { (Vioxx). Two trials } \\
\text { examining the } \\
\text { effects of Salix } \\
\text { Alba (White Willow } \\
\text { Bark) found } \\
\text { moderate evidence } \\
\text { that daily doses } \\
\text { standardized to } 120 \\
\text { mg or } 240 \text { mg } \\
\text { salicin were better } \\
\text { than placebo for } \\
\text { short-term } \\
\text { improvements in } \\
\text { pain and rescue } \\
\text { medication. An } \\
\text { additional trial } \\
\text { demonstrated }\end{array}$ & $\begin{array}{l}\text { en randomized } \\
\text { controlled trials that } \\
\text { included } 1567 \\
\text { adults with non- } \\
\text { specific acute or } \\
\text { chronic lowback } \\
\text { pain. Two oral } \\
\text { herbal medications, } \\
\text { Harpagophytum } \\
\text { Procumbens } \\
\text { (Devil's } \\
\text { Claw) and Salix } \\
\text { Alba (White Willow } \\
\text { Bark), were } \\
\text { compared with } \\
\text { placebo (fake pills) } \\
\text { and with rofecoxib } \\
\text { (Vioxx). Topical } \\
\text { Capsicum } \\
\text { frutescens } \\
\text { (Cayenne) was } \\
\text { compared with } \\
\text { placebo and a } \\
\text { homeopathic gel. }\end{array}$ \\
\hline
\end{tabular}




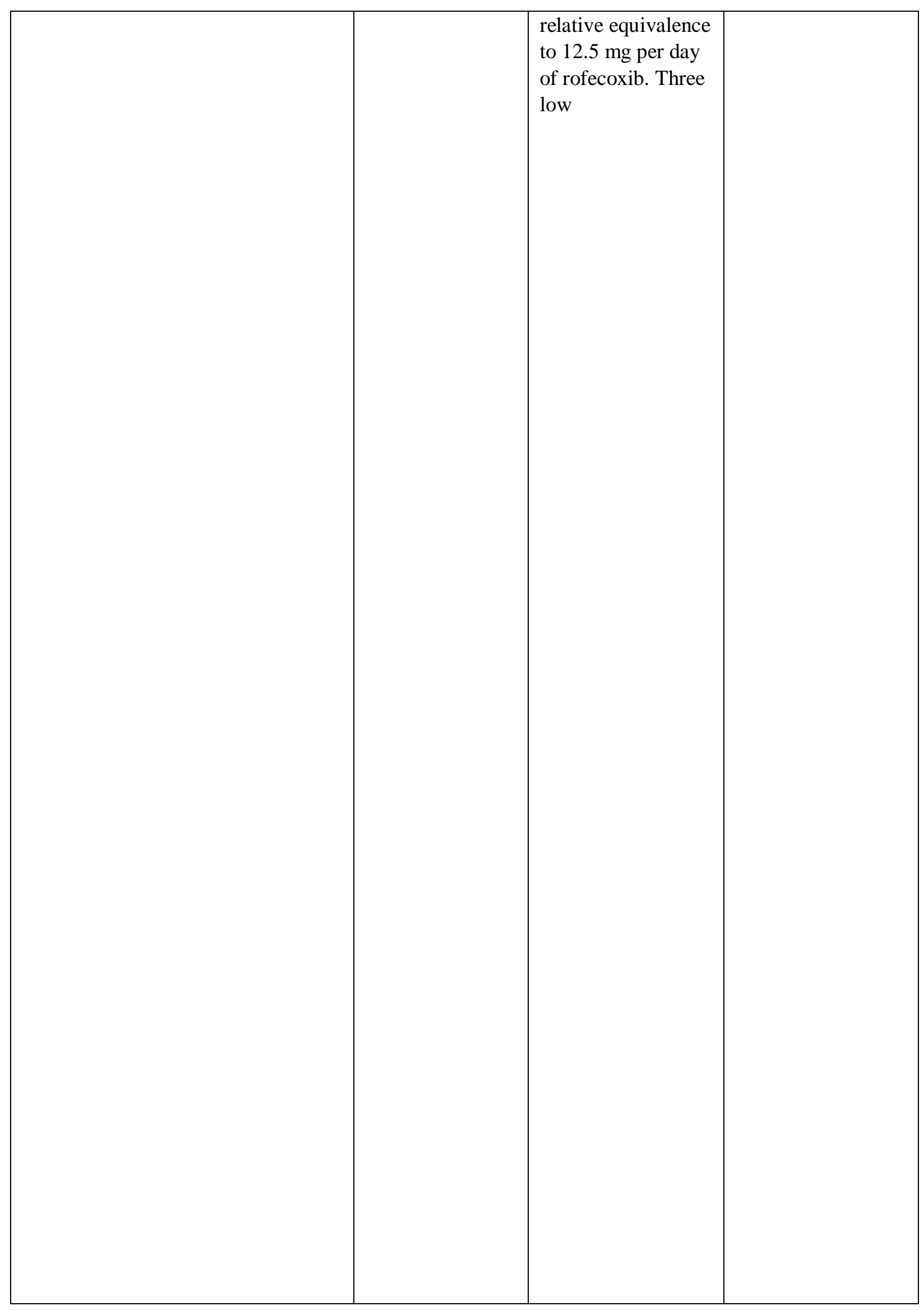




\begin{tabular}{|c|c|c|c|}
\hline & & $\begin{array}{l}\text { quality trials on } \\
\text { Capsicum } \\
\text { Frutescens } \\
\text { (Cayenne), } \\
\text { examining various } \\
\text { topical preparations, } \\
\text { found moderate } \\
\text { evidence that } \\
\text { Capsicum } \\
\text { Frutescens produced } \\
\text { more favourable } \\
\text { results than placebo } \\
\text { and one trial found } \\
\text { equivalence to a } \\
\text { homeopathic } \\
\text { ointment. }\end{array}$ & \\
\hline $\begin{array}{l}\text { Liu JP, Yang M, Liu Y, Wei ML, } \\
\text { Grimsgaard S. Herbal medicines for } \\
\text { treatment of irritable bowel } \\
\text { syndrome. Cochrane Database of } \\
\text { Systematic Reviews 2006, Issue } 1 . \\
\text { Art. No.: CD004116. } \\
\text { DOI: } \\
\text { 10.1002/14651858.CD00411 } \\
\text { 6.pub2 - See more at: } \\
\text { http://summaries.cochrane.or } \\
\text { g/CD004116/IBD_herbalmedicines- } \\
\text { for-treatment-ofirritable-bowel- } \\
\text { syndrome\#sthash.hU9phi70.d puf }\end{array}$ & $\begin{array}{l}\text { Various herbal } \\
\text { preparations for } \\
\text { IBS }\end{array}$ & $\begin{array}{l}\text { various herbal } \\
\text { preparations } \\
\text { (including single } \\
\text { herbs or mixtures of } \\
\text { different herbs) for } \\
\text { treating people with } \\
\text { irritable bowel } \\
\text { syndrome were } \\
\text { assessed. } \\
\text { individualised } \\
\text { Chinese herbal } \\
\text { medicine, STW } 5 \\
\text { and STW 5-II, } \\
\text { Tibetan herbal } \\
\text { medicine Padma } \\
\text { Lax, traditional } \\
\text { Chinese formula } \\
\text { Tongxie } \\
\text { Yaofang, and } \\
\text { Ayurvedic } \\
\text { preparation. some } \\
\text { herbal medicines } \\
\text { improve global } \\
\text { symptoms such as } \\
\text { abdominal pain, } \\
\text { diarrhoea and/or } \\
\text { constipation. } \\
\text { However, the } \\
\text { methodological } \\
\text { quality of the } \\
\text { majority of clinical }\end{array}$ & $\begin{array}{l}\text { Seventy-five } \\
\text { randomised trials, } \\
\text { involving } 7957 \\
\text { participants with } \\
\text { irritable bowel } \\
\text { syndrome, met the } \\
\text { inclusion criteria. } \\
\text { The methodological } \\
\text { quality of three } \\
\text { double-blind, } \\
\text { placebo-controlled } \\
\text { trials was high, but } \\
\text { the quality of } \\
\text { remaining trials was } \\
\text { generally low. } \\
\text { Seventy-one } \\
\text { different herbal } \\
\text { medicines were } \\
\text { tested in the } \\
\text { included trials, in } \\
\text { which herbal } \\
\text { medicines were } \\
\text { compared with } \\
\text { placebo or } \\
\text { conventional } \\
\text { pharmacologic } \\
\text { therapy. }\end{array}$ \\
\hline
\end{tabular}




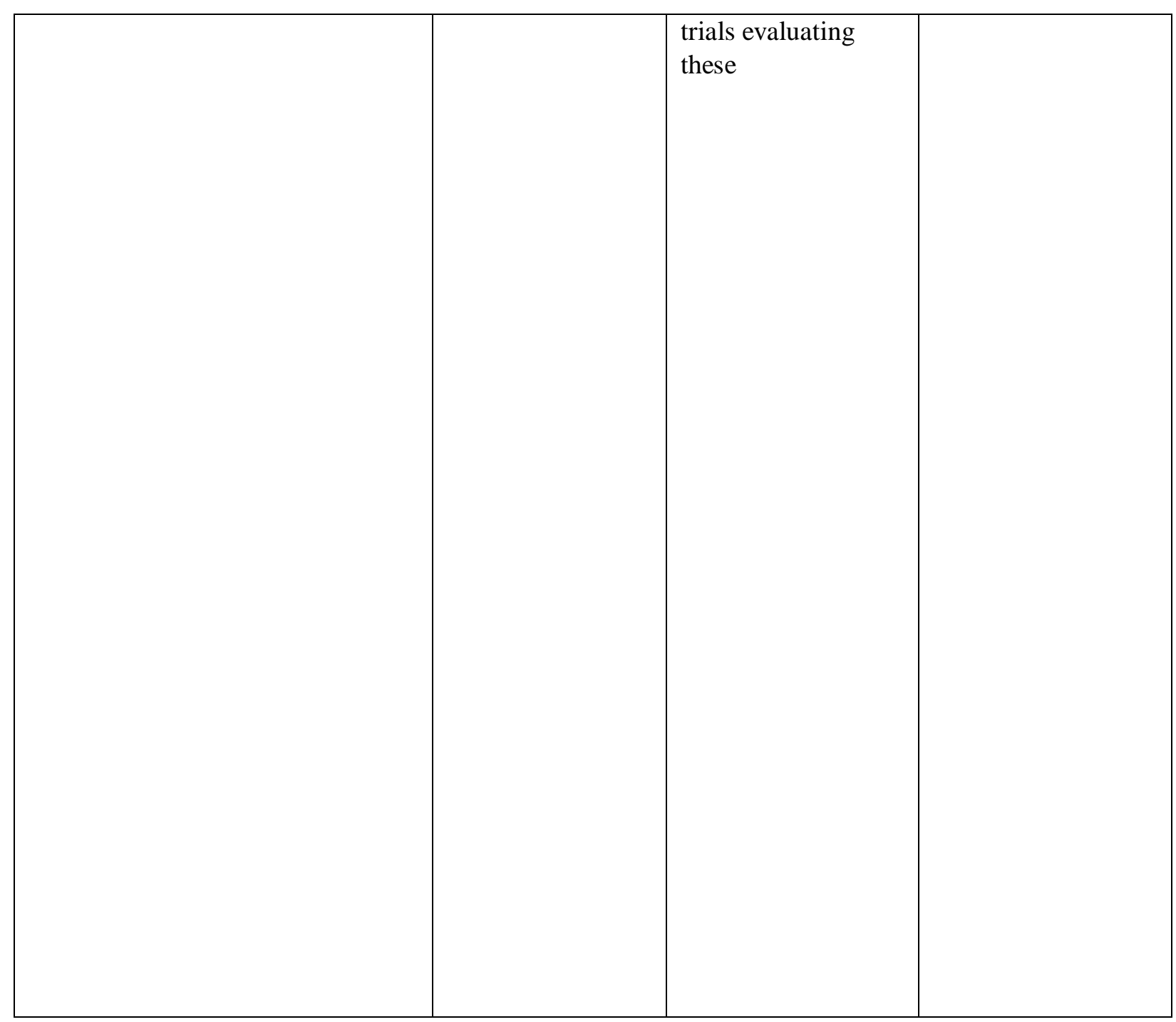




\begin{tabular}{|l|l|}
\hline & herbs was \\
generally poor. & There is evidence \\
indicating that & \\
small, poor quality & \\
trials with positive & findings are more \\
likely to be & associated with \\
exaggerated & effects. Although \\
the included trials \\
did not report \\
serious adverse \\
effects from using \\
herbal medicines \\
more research is \\
needed to \\
determine the \\
safety of herbal \\
medicines. In \\
conclusion, herbal \\
medicines might \\
be promising for \\
the treatment of \\
irritable bowel \\
syndrome. \\
\hline
\end{tabular}




\begin{tabular}{|c|c|c|c|}
\hline $\begin{array}{l}\text { Seidel S, Aigner M, Ossege } \\
\text { M, Pernicka E, Wildner B, Sycha T. } \\
\text { Antipsychotics for acute and chronic } \\
\text { pain in adults. Cochrane Database of } \\
\text { Systematic Reviews 2013, Issue } 8 . \\
\text { Art. No.: CD004844. } \\
\text { DOI: } \\
\text { 10.1002/14651858.CD00484 } \\
\text { 4.pub3 - See more at: } \\
\text { http://summaries.cochrane.or } \\
\text { g/CD004844/SYMPT_analge } \\
\text { sic-effects-of-antipsychoticsin- } \\
\text { acute-and-chronic- } \\
\text { painfulstates\#sthash.Xo1fwfPH.dpuf }\end{array}$ & antipsychotics & $\begin{array}{l}\text { Antipsychotics } \\
\text { might be used as } \\
\text { an add-on therapy } \\
\text { in the treatment of } \\
\text { painful conditions. } \\
\text { Nevertheless, } \\
\text { extrapyramidal } \\
\text { and sedating side } \\
\text { effects have to be } \\
\text { considered before } \\
\text { using } \\
\text { antipsychotics for } \\
\text { treating painful } \\
\text { conditions. } \\
\text { Results for } \\
\text { antipsychotics in } \\
\text { the treatment of } \\
\text { different painful } \\
\text { conditions are } \\
\text { mixed and most } \\
\text { sample sizes in } \\
\text { the reviewed } \\
\text { RCTs are small. } \\
\text { Further studies on } \\
\text { atypical } \\
\text { antipsychotics in } \\
\text { larger double- } \\
\text { blind placebo- } \\
\text { controlled studies } \\
\text { that include } \\
\text { standardised pain } \\
\text { assessment and }\end{array}$ & $\begin{array}{l}\text { A total of } 770 \\
\text { participants were } \\
\text { involved in the } 11 \\
\text { included studies. - } \\
\text { See more at: } \\
\text { http://summaries.coch } \\
\text { rane.org/CD004844/S } \\
\text { YMPT_analgesic- } \\
\text { effects- } \\
\text { ofantipsychotics- } \\
\text { inacute-and- } \\
\text { chronicpainful- } \\
\text { states\#sthash.Xo1fwf } \\
\text { PH.dpuf }\end{array}$ \\
\hline
\end{tabular}

\begin{tabular}{|l|l|l|l|}
\hline & & $\begin{array}{l}\text { documentation are } \\
\text { warranted. }\end{array}$ & \\
& & & \\
& & & \\
\hline
\end{tabular}




\begin{tabular}{|c|c|c|c|}
\hline $\begin{array}{l}\text { Moore RA, Straube S, Wiffen } \\
\text { PJ, Derry S, McQuay HJ. } \\
\text { Pregabalin for acute and } \\
\text { chronic pain in adults. } \\
\text { Cochrane Database of } \\
\text { Systematic Reviews 2009, } \\
\text { Issue 3. Art. No.: CD007076. } \\
\text { DOI: } \\
\text { 10.1002/14651858.CD00707 } \\
\text { 6.pub2 - See more at: } \\
\text { http://summaries.cochrane.or } \\
\text { g/CD007076/SYMPT_pregab } \\
\text { alin-for-acute-and- } \\
\text { chronicpain-in- } \\
\text { adults\#sthash.6lvmfRNw.dpu } \\
\text { f }\end{array}$ & $\begin{array}{l}\text { Antiepileptics (such } \\
\text { as pregabalin) are } \\
\text { medicines used for } \\
\text { treating epilepsy - } \\
\text { See more at: }\end{array}$ & $\begin{array}{l}\text { Pregabalin at doses of } \\
300 \text { mg, } 450 \text { mg, and } \\
600 \text { mg daily was } \\
\text { effective in patients } \\
\text { with postherpetic } \\
\text { neuralgia, painful } \\
\text { diabetic neuropathy, } \\
\text { central neuropathic } \\
\text { pain, and } \\
\text { fibromyalgia (19 } \\
\text { studies, } 7003 \\
\text { participants). } \\
\text { Pregabalin at } 150 \text { mg } \\
\text { daily was generally } \\
\text { ineffective. Efficacy } \\
\text { was demonstrated for } \\
\text { dichotomous } \\
\text { outcomes equating to } \\
\text { moderate or } \\
\text { substantial pain relief, } \\
\text { alongside lower rates } \\
\text { for lack of efficacy } \\
\text { discontinuations with } \\
\text { increasing dose. The } \\
\text { best (lowest) NNT for } \\
\text { each condition for at } \\
\text { least } 50 \% \text { pain relief } \\
\text { over baseline } \\
\text { (substantial benefit) } \\
\text { for } 600 \text { mg } \\
\text { pregabalin daily } \\
\text { compared with } \\
\text { placebo were } 3.9 \\
\text { (95\% confidence } \\
\text { interval } 3.1 \text { to } 5.1 \text { ) for } \\
\text { postherpetic } \\
\text { neuralgia, } 5.0 \text { (4.0 to } \\
6.6 \text { ) for painful } \\
\text { diabetic neuropathy, }\end{array}$ & not stated \\
\hline
\end{tabular}




\begin{tabular}{|c|c|c|c|}
\hline & & $\begin{array}{l}5.6 \text { ( } 3.5 \text { to } 14 \text { ) for } \\
\text { central neuropathic } \\
\text { pain, and } 11 \text { ( } 7.1 \text { to } \\
21 \text { ) for } \\
\text { fibromyalgia. There } \\
\text { was no clear } \\
\text { evidence of } \\
\text { beneficial effects of } \\
\text { pregabalin in } \\
\text { established acute } \\
\text { postoperative pain. } \\
\text { No studies } \\
\text { evaluated } \\
\text { pregabalin in } \\
\text { chronic nociceptive } \\
\text { pain, like arthritis. } \\
\text { Pregabalin has } \\
\text { proven efficacy in } \\
\text { neuropathic pain } \\
\text { conditions and } \\
\text { fibromyalgia. }\end{array}$ & \\
\hline $\begin{array}{l}\text { Williams AC de C, Eccleston C, } \\
\text { Morley S. Psychological } \\
\text { therapies for the management of } \\
\text { chronic pain (excluding } \\
\text { headache) in adults. Cochrane } \\
\text { Database of Systematic } \\
\text { Reviews 2012, Issue 11. Art. } \\
\text { No.: CD007407. DOI: } \\
\text { 10.1002/14651858.CD00740 } \\
\text { 7.pub3 - See more at: } \\
\text { http://summaries.cochrane.or } \\
\text { g/CD007407/SYMPT_psycho } \\
\text { logical-therapy-for-adultswith- } \\
\text { longstandingdistressing-pain- } \\
\text { anddisability\#sthash.B2SouCUv. } \\
\text { dpuf }\end{array}$ & $\begin{array}{l}\text { CBT and behavior } \\
\text { therapy }\end{array}$ & $\begin{array}{l}\text { Small to moderate } \\
\text { benefits, more for } \\
\text { disability, mood and } \\
\text { catastrophic } \\
\text { thinking than for } \\
\text { pain, were found in } \\
\text { trials which } \\
\text { compared CBT with } \\
\text { no treatment. Some } \\
\text { of these were still } \\
\text { positive six months } \\
\text { later. Behaviour } \\
\text { therapy showed few } \\
\text { and only brief } \\
\text { benefits. } \\
\text { Psychological } \\
\text { therapies can help } \\
\text { people with chronic } \\
\text { pain reduce negative } \\
\text { mood (depression } \\
\text { and anxiety), } \\
\text { disability, } \\
\text { catastrophic } \\
\text { thinking, and in } \\
\text { some cases, pain. } \\
\text { Although the overall } \\
\text { effect is positive, we } \\
\text { do not know enough } \\
\text { about exactly which }\end{array}$ & $\begin{array}{l}42 \text { trials of treatments } \\
\text { which met our criteria, } \\
\text { but only } 35 \text { provided } \\
\text { data in a form that could } \\
\text { be used. The two main } \\
\text { types of psychological } \\
\text { treatment are called } \\
\text { cognitive behavioural } \\
\text { therapy (CBT) and } \\
\text { behaviour therapy. - See } \\
\text { more at: } \\
\text { http://summaries.coch } \\
\text { rane.org/CD007407/S } \\
\text { YMPT_psychological } \\
\text {-therapy-for-adultswith- } \\
\text { longstandingdistressing- } \\
\text { pain- } \\
\text { anddisability\#sthash.B2S } \\
\text { ouCUv.dpuf }\end{array}$ \\
\hline
\end{tabular}




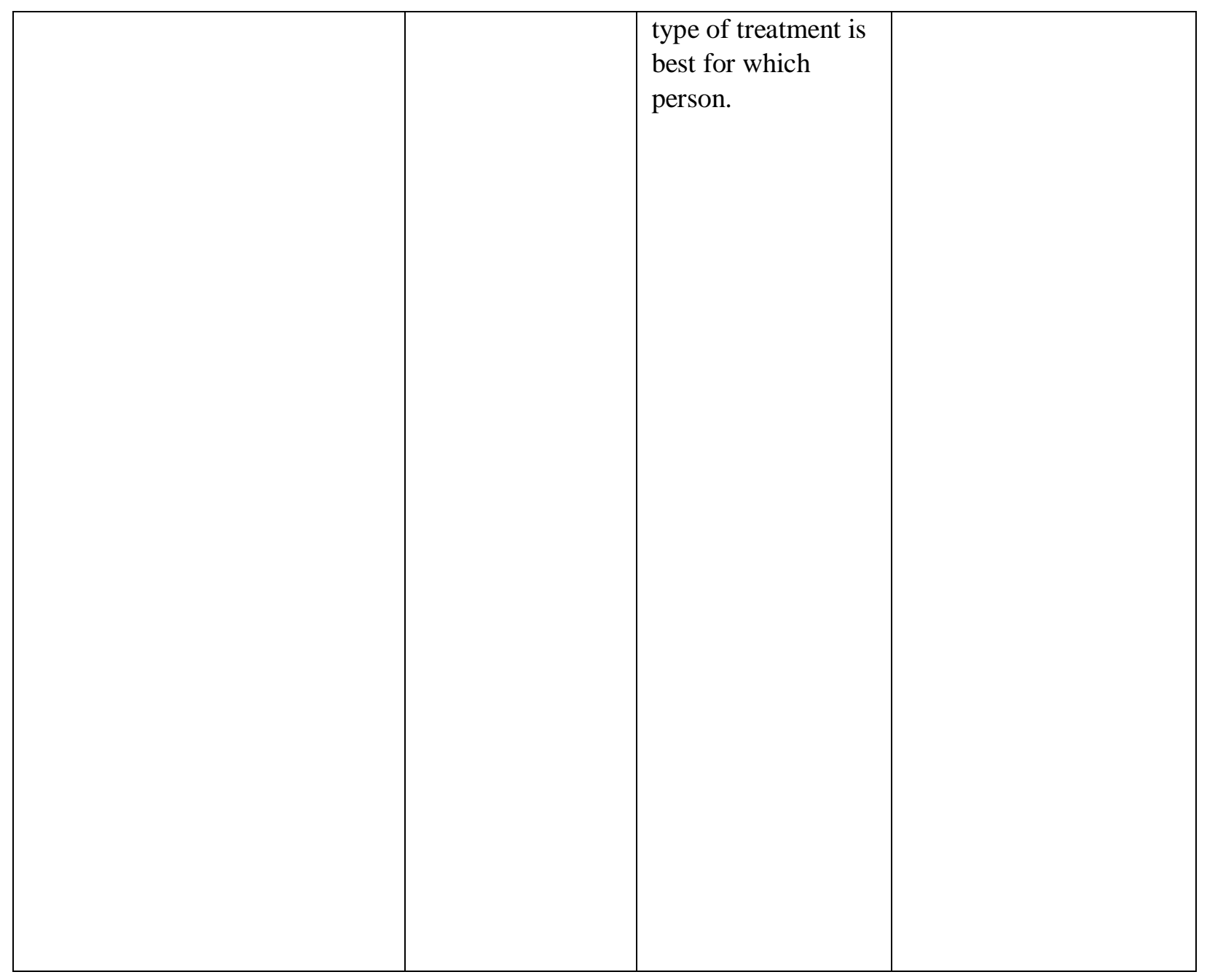

\begin{tabular}{|c|c|c|c|}
\hline $\begin{array}{l}\text { Matthews P, Derry S, Moore } \\
\text { RA, McQuay HJ. Topical } \\
\text { rubefacients for acute and } \\
\text { chronic pain in adults. } \\
\text { Cochrane Database of } \\
\text { Systematic Reviews 2009, Issue } \\
\text { 3. Art. No.: CD007403. } \\
\text { DOI: } \\
\text { 10.1002/14651858.CD00740 } \\
\text { 3.pub2 - See more at: } \\
\text { http://summaries.cochrane.or } \\
\text { g/CD007403/SYMPT_topical } \\
\text {-rubefacients-for-acute- } \\
\text { andchronic- } \\
\text { musculoskeletalpain-in- } \\
\text { adults\#sthash.TXMzmN3h.dp uf }\end{array}$ & topical salicylates & $\begin{array}{l}\text { The evidence does } \\
\text { not support the use of } \\
\text { topical rubefacients } \\
\text { containing salicylates } \\
\text { for acute injuries, and } \\
\text { suggests that in } \\
\text { chronic conditions } \\
\text { their efficacy } \\
\text { compares poorly with } \\
\text { topical non-steroidal } \\
\text { antiinflammatory } \\
\text { drugs (NSAIDs). } \\
\text { Topical salicylates } \\
\text { seem to be relatively } \\
\text { well tolerated in the } \\
\text { short-term, based on } \\
\text { limited data. }\end{array}$ & $\begin{array}{l}\text { Six placebo and one } \\
\text { active controlled } \\
\text { studies (560 and } 137 \\
\text { participants) in acute } \\
\text { pain, and seven } \\
\text { placebo and two } \\
\text { active controlled } \\
\text { studies ( } 489 \text { and } 90 \\
\text { participants) in } \\
\text { chronic pain were } \\
\text { included. }\end{array}$ \\
\hline
\end{tabular}




\begin{tabular}{|c|c|c|c|}
\hline $\begin{array}{l}\text { Eccleston C, Fisher E, Craig } \\
\text { L, Duggan GB, Rosser BA, } \\
\text { Keogh E. Psychological } \\
\text { therapies (Internet-delivered) for } \\
\text { the management of chronic pain } \\
\text { in adults. } \\
\text { Cochrane Database of } \\
\text { Systematic Reviews 2014, Issue } \\
\text { 2. Art. No.: CD010152. } \\
\text { DOI: } \\
\text { 10.1002/14651858.CD01015 } \\
\text { 2.pub2 - See more at: } \\
\text { http://summaries.cochrane.or } \\
\text { g/CD010152/SYMPT_psycho } \\
\text { logical-therapies-deliveredvia- } \\
\text { the-internet-for-adultswith- } \\
\text { longstandingdistressing-pain- } \\
\text { anddisability\#sthash.uFGoYr5V. } \\
\text { dpuf }\end{array}$ & $\begin{array}{l}\text { Psychological } \\
\text { therapies (e.g. } \\
\text { cognitive } \\
\text { behavioural therapy) } \\
\text { delivered via the } \\
\text { internet }\end{array}$ & $\begin{array}{l}\text { We conclude that } \\
\text { these findings are } \\
\text { promising for } \\
\text { psychological } \\
\text { treatments delivered } \\
\text { via the Internet for } \\
\text { the management of } \\
\text { chronic pain in } \\
\text { adults, but more trials } \\
\text { are needed to } \\
\text { determine the } \\
\text { efficacy of such } \\
\text { therapies. }\end{array}$ & $\begin{array}{l}15 \text { trials that met our } \\
\text { inclusion criteria. } \\
\text { Four trials included } \\
\text { individuals with } \\
\text { headache pain, } 10 \\
\text { trials included } \\
\text { individuals with } \\
\text { nonheadache pain, } \\
\text { and one trial included } \\
\text { individuals with both } \\
\text { headache and } \\
\text { nonheadache pain. }\end{array}$ \\
\hline
\end{tabular}

\begin{tabular}{|c|c|c|c|}
\hline $\begin{array}{l}\text { Miyasaka LS, Atallah ÁN, } \\
\text { Soares B. Passiflora for } \\
\text { anxiety disorder. Cochrane } \\
\text { Database of Systematic } \\
\text { Reviews 2007, Issue 1. Art. } \\
\text { No.: CD004518. DOI: } \\
\text { 10.1002/14651858.CD00451 } \\
\text { 8.pub2 - See more at: } \\
\text { http://summaries.cochrane.or } \\
\text { g/CD004518/DEPRESSN_pa } \\
\text { ssiflora-for-the-treatment- } \\
\text { ofanxiety-disorders- } \\
\text { inadults\#sthash.pA0IREr6.dpuf }\end{array}$ & $\begin{array}{l}\text { Passiflora, a herbal } \\
\text { medicine }\end{array}$ & $\begin{array}{l}\text { Given the lack of } \\
\text { studies, it is not } \\
\text { possible to draw any } \\
\text { conclusions on the } \\
\text { effectiveness or } \\
\text { safety of passiflora in } \\
\text { the treatment of } \\
\text { anxiety disorders. } \\
\text { Although the findings } \\
\text { from one study } \\
\text { suggested an } \\
\text { improvement in job } \\
\text { performance in } \\
\text { favour of passiflora } \\
\text { (post-hoc outcome) } \\
\text { and one study } \\
\text { showed a lower rate } \\
\text { of drowsiness as a } \\
\text { side effect with } \\
\text { passiflora as } \\
\text { compared with } \\
\text { mexazolam, neither } \\
\text { of these findings } \\
\text { reached statistical } \\
\text { significance. }\end{array}$ & $\begin{array}{l}\text { Two studies, with a } \\
\text { total of } 198 \\
\text { participants, were } \\
\text { eligible for inclusion } \\
\text { in this review. Used } \\
\text { Relevant randomised } \\
\text { and quasi-randomised } \\
\text { controlled trials of } \\
\text { passiflora using any } \\
\text { dose, regime, or } \\
\text { method of } \\
\text { administration for } \\
\text { people with any } \\
\text { primary diagnosis of } \\
\text { general anxiety } \\
\text { disorder, anxiety } \\
\text { neurosis, chronic } \\
\text { anxiety status or any } \\
\text { other mental health } \\
\text { disorder in which } \\
\text { anxiety is a core } \\
\text { symptom (panic } \\
\text { disorder, obsessive } \\
\text { compulsive disorder, } \\
\text { social phobia, } \\
\text { agoraphobia, other } \\
\text { types of phobia, } \\
\text { postraumatic stress } \\
\text { disorder). }\end{array}$ \\
\hline
\end{tabular}




\begin{tabular}{|c|c|c|c|}
\hline $\begin{array}{l}\text { Miyasaka LS, Atallah ÁN, Soares B. } \\
\text { Valerian for anxiety disorders. } \\
\text { Cochrane Database of Systematic } \\
\text { Reviews 2006, Issue 4. Art. } \\
\text { No.: CD004515. DOI: } \\
\text { 10.1002/14651858.CD00451 } \\
\text { 5.pub2 - See more at: } \\
\text { http://summaries.cochrane.or } \\
\text { g/CD004515/DEPRESSN_va lerian- } \\
\text { for- } \\
\text { anxietydisorders\#sthash.3dwXF2Hx. } \\
\text { dpuf }\end{array}$ & $\begin{array}{l}\text { Valerian, an } \\
\text { herbal remedy }\end{array}$ & $\begin{array}{l}\text { Since only one } \\
\text { small study is } \\
\text { currently available, } \\
\text { there is insufficient } \\
\text { evidence to draw } \\
\text { any conclusions } \\
\text { about the efficacy or } \\
\text { safety of valerian } \\
\text { compared with } \\
\text { placebo or } \\
\text { diazepam for } \\
\text { anxiety disorders. } \\
\text { RCTs involving } \\
\text { larger samples and } \\
\text { comparing valerian } \\
\text { with placebo or } \\
\text { other interventions } \\
\text { used to treat of } \\
\text { anxiety disorders, } \\
\text { such as } \\
\text { antidepressants, are } \\
\text { needed. }\end{array}$ & $\begin{array}{l}\text { One RCT involving } \\
36 \text { patients with } \\
\text { generalised anxiety } \\
\text { disorder was eligible } \\
\text { for inclusion. This } \\
\text { was a } 4 \text { week pilot } \\
\text { study of valerian, } \\
\text { diazepam and } \\
\text { placebo. search was } \\
\text { Randomised } \\
\text { controlled trials } \\
\text { (RCTs) and } \\
\text { quasirandomised } \\
\text { trials of valerian } \\
\text { extract of any dose, } \\
\text { regime, or method } \\
\text { of administration, } \\
\text { for people with any } \\
\text { primary diagnosis of } \\
\text { general anxiety } \\
\text { disorder, anxiety } \\
\text { neurosis, chronic } \\
\text { anxiety status, or } \\
\text { any other disorder in } \\
\text { which anxiety is the } \\
\text { primary symptom } \\
\text { (panic disorder, } \\
\text { obsessive } \\
\text { compulsive } \\
\text { disorder, social } \\
\text { phobia, } \\
\text { agoraphobia, other } \\
\text { types of phobia, } \\
\text { postraumatic stress } \\
\text { disorder). } \\
\text { Effectiveness was } \\
\text { measured using } \\
\text { clinical outcome } \\
\text { measures and other } \\
\text { scales for anxiety } \\
\text { symptoms. }\end{array}$ \\
\hline
\end{tabular}




\begin{tabular}{|c|c|c|c|}
\hline $\begin{array}{l}\text { Cameron M, Gagnier JJ, Chrubasik S. } \\
\text { Herbal therapy for treating rheumatoid } \\
\text { arthritis. Cochrane Database of } \\
\text { Systematic Reviews 2011, Issue 2. Art. } \\
\text { No.: CD002948. } \\
\text { DOI: } \\
\text { 10.1002/14651858.CD00294 } \\
\text { 8.pub2 - See more at: } \\
\text { http://summaries.cochrane.or } \\
\text { g/CD002948/MUSKEL_herb } \\
\text { al-therapy-for- } \\
\text { rheumatoidarthritis\#sthash.dFv5Qoew.dp } \\
\text { uf }\end{array}$ & $\begin{array}{l}\text { Herbal } \\
\text { interventions are } \\
\text { defined as any } \\
\text { plant preparation } \\
\text { (whole, powder, } \\
\text { extract, } \\
\text { standardised } \\
\text { mixture) used } \\
\text { for medicinal } \\
\text { purposes. . RA, } \\
\text { your immune } \\
\text { system, which } \\
\text { normally fights } \\
\text { infection, attacks } \\
\text { the lining of } \\
\text { your joints. This } \\
\text { makes your } \\
\text { joints swollen, } \\
\text { stiff and painful. } \\
\text { The small joints } \\
\text { of your hands } \\
\text { and feet are } \\
\text { usually affected } \\
\text { first. There is no } \\
\text { cure for } \\
\text { rheumatoid } \\
\text { arthritis at } \\
\text { present, so } \\
\text { treatments are } \\
\text { used to relieve } \\
\text { pain and } \\
\text { stiffness and } \\
\text { improve your } \\
\text { ability to move. }\end{array}$ & $\begin{array}{l}\text { Several herbal } \\
\text { interventions are } \\
\text { inadequately } \\
\text { justified by single } \\
\text { studies or } \\
\text { non-comparable } \\
\text { studies in the } \\
\text { treatment of } \\
\text { rheumatoid arthritis. } \\
\text { There is moderate } \\
\text { evidence that oils } \\
\text { containing GLA } \\
\text { (evening primrose, } \\
\text { borage, or } \\
\text { blackcurrant seed oil) } \\
\text { afford some benefit in } \\
\text { relieving symptoms } \\
\text { for RA, while } \\
\text { evidence for } \\
\text { Phytodolor® N is } \\
\text { less } \\
\text { convincing.Tripterygi } \\
\text { um wilfordii products } \\
\text { may reduce some RA } \\
\text { symptoms, however, } \\
\text { oral use may be } \\
\text { associated with } \\
\text { several side effects. } \\
\text { Many trials of herbal } \\
\text { therapies are } \\
\text { hampered by research } \\
\text { design flaws and } \\
\text { inadequate reporting. } \\
\text { Further investigation } \\
\text { of each herbal } \\
\text { therapy is warranted, } \\
\text { particularly via well } \\
\text { designed, fully } \\
\text { powered, } \\
\text { confirmatory clinical } \\
\text { trials that use } \\
\text { American College of } \\
\text { Rheumatology } \\
\text { improvement criteria } \\
\text { to measure outcomes } \\
\text { and report results } \\
\text { according to } \\
\text { CONSORT } \\
\text { guidelines. }\end{array}$ & $\begin{array}{l}\text { Twelve new } \\
\text { studies were } \\
\text { added to the } \\
\text { update, a total } \\
\text { of } 22 \text { studies } \\
\text { were included. }\end{array}$ \\
\hline
\end{tabular}




\begin{tabular}{|c|c|c|c|}
\hline $\begin{array}{l}\text { Cramp F, Hewlett S, Almeida } \\
\text { C, Kirwan JR, Choy EHS, } \\
\text { Chalder T, Pollock J, Christensen R. Nonpharmacological } \\
\text { interventions for fatigue in rheumatoid arthritis. } \\
\text { Cochrane Database of } \\
\text { Systematic Reviews 2013, Issue 8. Art. No.: CD008322. } \\
\text { DOI: } \\
\text { 10.1002/14651858.CD00832 } \\
\text { 2.pub2 - See more at: } \\
\text { http://summaries.cochrane.or } \\
\text { g/CD008322/MUSKEL_nonpharmacologicalinterventions- } \\
\text { for-themanagement-of-patientreported-fatigue- } \\
\text { inrheumatoid- } \\
\text { arthritis\#sthash.iSsGqRK9.dp uf }\end{array}$ & $\begin{array}{l}\text { Physical } \\
\text { activity and } \\
\text { psychosocial } \\
\text { therapy } \\
\text { for Fatigue } \\
\text { is a common } \\
\text { and } \\
\text { potentially } \\
\text { distressing } \\
\text { symptom for } \\
\text { people with } \\
\text { rheumatoid } \\
\text { arthritis with } \\
\text { no accepted } \\
\text { evidence } \\
\text { based } \\
\text { management } \\
\text { guidelines. }\end{array}$ & $\begin{array}{l}\text { This review provides } \\
\text { some evidence that } \\
\text { physical activity and } \\
\text { psychosocial } \\
\text { interventions provide } \\
\text { benefit in relation to } \\
\text { self-reported fatigue } \\
\text { in adults with } \\
\text { rheumatoid arthritis. } \\
\text { There is currently } \\
\text { insufficient evidence } \\
\text { of the effectiveness } \\
\text { of } \\
\text { other } \\
\text { nonpharmacological } \\
\text { interventions. } \\
\text { Physical activity } \\
\text { has a small benefit } \\
\text { for managing } \\
\text { fatigue in people } \\
\text { with rheumatoid } \\
\text { arthritis. } \\
\text { specifically: - } \\
\text { Psychosocial } \\
\text { therapy has a small } \\
\text { benefit for } \\
\text { managing fatigue in } \\
\text { people with } \\
\text { rheumatoid } \\
\text { arthritis. } \\
\text { - No other } \\
\text { interventions showed } \\
\text { a difference in } \\
\text { managing fatigue in } \\
\text { people with } \\
\text { rheumatoid arthritis. } \\
\text { This may have } \\
\text { happened by chance. }\end{array}$ & $\begin{array}{l}24 \text { were } \\
\text { identified for } \\
\text { inclusion in the } \\
\text { review with a } \\
\text { total of } 2882 \\
\text { people. } \\
\text { Specifically, } \\
\text { physical } \\
\text { activity } \\
\text { interventions (n } \\
=6 \text { studies; } 388 \\
\text { participants), } \\
\text { psychosocial } \\
\text { interventions (n } \\
=13 \text { studies; } \\
1579 \\
\text { participants), } \\
\text { herbal medicine } \\
\text { (n = } 1 \text { study; } 58 \\
\text { participants), } \\
\text { omega- } \\
3 \text { fatty acid } \\
\text { supplementation } \\
\text { (n = } \\
1 \text { study; } 81 \\
\text { participants), } \\
\text { Mediterranean } \\
\text { diet (n } \\
=1 \text { study; } 51 \\
\text { participants), } \\
\text { reflexology (n } \\
=1 \text { study; } 11 \\
\text { participants) } \\
\text { and the } \\
\text { provision of } \\
\text { Health Tracker } \\
\text { information (n } \\
=1 \text { study; } 714 \\
\text { participants). }\end{array}$ \\
\hline
\end{tabular}




\begin{tabular}{|c|c|c|c|}
\hline $\begin{array}{l}\text { Pittler MH, Ernst E. Feverfew for preventing migraine. } \\
\text { Cochrane Database of Systematic Reviews 2004, Issue } 1 . \\
\text { Art. No.: CD002286. } \\
\text { DOI: } \\
\text { 10.1002/14651858.CD00228 } \\
\text { 6.pub2 - See more at: } \\
\text { http://summaries.cochrane.or } \\
\text { g/CD002286/SYMPT_feverfe w-for- } \\
\text { preventingmigraine\#sthash.UolN9Eue.d puf }\end{array}$ & Feverfew & $\begin{array}{l}\text { Results from these } \\
\text { trials were mixed } \\
\text { and did not } \\
\text { convincingly } \\
\text { establish that } \\
\text { feverfew is } \\
\text { efficacious for } \\
\text { preventing migraine. } \\
\text { There is insufficient } \\
\text { evidence from } \\
\text { randomised, } \\
\text { doubleblind trials to } \\
\text { suggest an effect of } \\
\text { feverfew over and } \\
\text { above placebo for } \\
\text { preventing migraine. }\end{array}$ & $\begin{array}{l}\text { Five trials ( } 343 \\
\text { patients) met } \\
\text { the inclusion } \\
\text { criteria. } \\
\text { Randomised, } \\
\text { placebo- } \\
\text { controlled, } \\
\text { double-blind } \\
\text { trials assessing } \\
\text { the efficacy of } \\
\text { feverfew for } \\
\text { preventing } \\
\text { migraine were } \\
\text { included. }\end{array}$ \\
\hline
\end{tabular}

\begin{tabular}{|c|c|c|c|}
\hline $\begin{array}{l}\text { Pittler MH, Ernst E. Kava extract } \\
\text { versus placebo for treating anxiety. } \\
\text { Cochrane Database of Systematic } \\
\text { Reviews 2003, Issue 1. Art. } \\
\text { No.: CD003383. DOI: } \\
\text { 10.1002/14651858.CD00338 } \\
\text { 3- See more at: } \\
\text { http://summaries.cochrane.or } \\
\text { g/CD003383/DEPRESSN_ka va- } \\
\text { extract-for- } \\
\text { treatinganxiety\#sthash.vk8QIdpU.dp } \\
\text { uf }\end{array}$ & kava for anxiety & $\begin{array}{l}\text { The meta-analysis } \\
\text { of seven trials } \\
\text { suggests a } \\
\text { significant treatment } \\
\text { effect for the total } \\
\text { score on the } \\
\text { Hamilton Anxiety } \\
\text { Scale in favour of } \\
\text { kava extract. Few } \\
\text { adverse events were } \\
\text { reported in the } \\
\text { reviewed trials, } \\
\text { which were all mild, } \\
\text { transient and } \\
\text { infrequent. These } \\
\text { data imply that, } \\
\text { compared with } \\
\text { placebo, kava } \\
\text { extract might be an } \\
\text { effective } \\
\text { symptomatic } \\
\text { treatment for } \\
\text { anxiety although, at } \\
\text { present, the size of } \\
\text { the effect seems to } \\
\text { be small. - }\end{array}$ & $\begin{array}{l}\text { Twenty-two } \\
\text { potentially relevant } \\
\text { double-blind, } \\
\text { placebo-controlled } \\
\text { RCTs were } \\
\text { identified. Twelve } \\
\text { trials met the } \\
\text { inclusion criteria } \\
((\mathrm{n}=700)) \text {. }\end{array}$ \\
\hline
\end{tabular}




\begin{tabular}{|c|c|c|c|}
\hline $\begin{array}{l}\text { Richards BL, Whittle SL, Buchbinder R. } \\
\text { Neuromodulators for pain management } \\
\text { in rheumatoid arthritis. Cochrane } \\
\text { Database of Systematic Reviews 2012, } \\
\text { Issue 1. Art. No.: CD008921. } \\
\text { DOI: } \\
\text { 10.1002/14651858.CD00892 } \\
\text { 1.pub2 - See more at: } \\
\text { http://summaries.cochrane.or } \\
\text { g/CD008921/MUSKEL_neur } \\
\text { omodulators-for-painmanagement-in- } \\
\text { rheumatoidarthritis\#sthash.NqAtL4XY.d } \\
\text { puf }\end{array}$ & $\begin{array}{l}\text { neuromodulators } \\
\text { (broadly defined } \\
\text { as substances } \\
\text { which alter the } \\
\text { way nerves } \\
\text { communicate with } \\
\text { each other and, } \\
\text { consequently, the } \\
\text { overall activity } \\
\text { level of the brain. } \\
\text { By acting on these } \\
\text { nerve signals it is } \\
\text { thought that these } \\
\text { drugs can reduce } \\
\text { the amount of } \\
\text { pain felt by an } \\
\text { individual. } \\
\text { Neuromodulators } \\
\text { sometimes used in } \\
\text { pain management } \\
\text { and assessed here } \\
\text { include } \\
\text { anticonvulsants } \\
\text { (gabapentin, } \\
\text { pregabalin, } \\
\text { phenytoin, sodium } \\
\text { valproate, } \\
\text { lamotrigine, } \\
\text { carbamazepine, } \\
\text { levetiracetam, } \\
\text { oxcarbazepine, } \\
\text { tiagabine and } \\
\text { topiramate), } \\
\text { ketamine, } \\
\text { bupropion, } \\
\text { methylphenidate, } \\
\text { nefopam, } \\
\text { capsaicin and the } \\
\text { cannabinoids.) for } \\
\text { rhuematoid } \\
\text { arthritis }\end{array}$ & $\begin{array}{l}\text { There is } \\
\text { currently weak } \\
\text { evidence that } \\
\text { oral nefopam, } \\
\text { topical } \\
\text { capsaicin and } \\
\text { oromucosal } \\
\text { cannabis are all } \\
\text { superior to } \\
\text { placebo in } \\
\text { reducing pain } \\
\text { in patients with } \\
\text { RA. However, } \\
\text { each agent is } \\
\text { associated with } \\
\text { a significant } \\
\text { side effect } \\
\text { profile. The } \\
\text { confidence in } \\
\text { our estimates is } \\
\text { not strong } \\
\text { given the } \\
\text { difficulties } \\
\text { with blinding, } \\
\text { the small } \\
\text { numbers of } \\
\text { participants } \\
\text { evaluated and } \\
\text { the lack of } \\
\text { adverse event } \\
\text { data. In some } \\
\text { patients, } \\
\text { however, even } \\
\text { a small degree } \\
\text { of pain relief } \\
\text { may be } \\
\text { considered } \\
\text { worthwhile. } \\
\text { Until further } \\
\text { research is } \\
\text { available, } \\
\text { given the } \\
\text { relatively mild } \\
\text { nature of the } \\
\text { adverse events, } \\
\text { capsaicin could } \\
\text { be considered } \\
\text { as an addon } \\
\text { therapy for } \\
\text { patients with } \\
\text { persistent local }\end{array}$ & $\begin{array}{l}\text { We included } \\
\text { randomised } \\
\text { controlled trials } \\
\text { which compared any } \\
\text { neuromodulator to } \\
\text { another therapy } \\
\text { (active or placebo, } \\
\text { including } \\
\text { nonpharmacological } \\
\text { therapies) in adult } \\
\text { patients with RA } \\
\text { that had at least one } \\
\text { clinically relevant } \\
\text { outcome } \\
\text { measure.Four trials } \\
\text { with high risk of bias } \\
\text { were included in this } \\
\text { review. Two trials } \\
\text { evaluated oral } \\
\text { nefopam (52 } \\
\text { participants) and one } \\
\text { trial each evaluated } \\
\text { topical capsaicin ( } 31 \\
\text { participants) and } \\
\text { oromucosal cannabis } \\
\text { (58 participants). }\end{array}$ \\
\hline
\end{tabular}




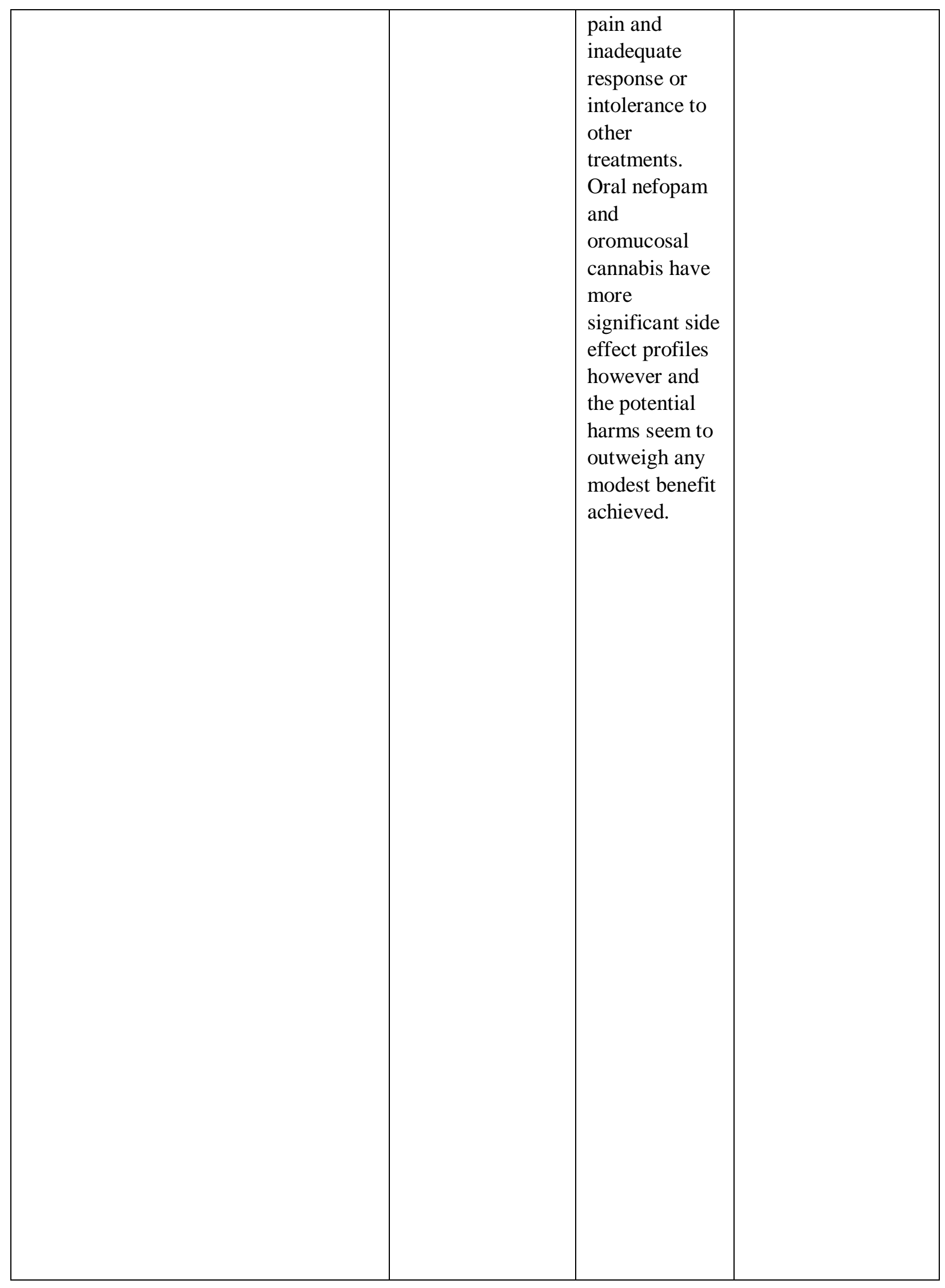




\begin{tabular}{|c|c|c|c|}
\hline $\begin{array}{l}\text { Linde K, Berner MM, Kriston L. St } \\
\text { John's wort for major depression. } \\
\text { Cochrane } \\
\text { Database of Systematic } \\
\text { Reviews 2008, Issue 4. Art. } \\
\text { No.: CD000448. DOI: } \\
\text { 10.1002/14651858.CD00044 } \\
\text { 8.pub3 - See more at: } \\
\text { http://summaries.cochrane.or } \\
\text { g/CD000448/DEPRESSN_st. } \\
\text {-johns-wort-for- } \\
\text { treatingdepression.\#sthash.cpTrZv0Y } \\
\text {.dpuf }\end{array}$ & $\begin{array}{l}\text { St JOHNS Wort } \\
\text { for depression }\end{array}$ & $\begin{array}{l}\text { The available } \\
\text { evidence suggests } \\
\text { that the hypericum } \\
\text { extracts tested in } \\
\text { the included trials } \\
\text { a) are superior to } \\
\text { placebo in patients } \\
\text { with major } \\
\text { depression; b) are } \\
\text { similarly effective } \\
\text { as standard } \\
\text { antidepressants; c) } \\
\text { and have fewer side } \\
\text { effects than } \\
\text { standard } \\
\text { antidepressants. The } \\
\text { association of } \\
\text { country of origin } \\
\text { and precision with } \\
\text { effects sizes } \\
\text { complicates the } \\
\text { interpretation. }\end{array}$ & $\begin{array}{l}29 \text { studies in } 5489 \\
\text { patients with } \\
\text { depression that } \\
\text { compared treatment } \\
\text { with extracts of St. } \\
\text { John's wort for } 4 \text { to } \\
12 \text { weeks with } \\
\text { placebo treatment or } \\
\text { standard } \\
\text { antidepressants. The } \\
\text { studies came from a } \\
\text { variety of countries, } \\
\text { tested several } \\
\text { different St. John's } \\
\text { wort extracts, and } \\
\text { mostly included } \\
\text { patients suffering } \\
\text { from mild to } \\
\text { moderately severe } \\
\text { symptoms. Trials } \\
\text { were included if } \\
\text { they: (1) were } \\
\text { randomised and } \\
\text { double-blind; (2) } \\
\text { included patients } \\
\text { with major } \\
\text { depression; } \\
\text { compared extracts } \\
\text { of St. John's wort } \\
\text { with placebo or } \\
\text { standard } \\
\text { antidepressants; (4) } \\
\text { included clinical } \\
\text { outcomes assessing } \\
\text { depressive } \\
\text { symptoms. }\end{array}$ \\
\hline
\end{tabular}




\begin{tabular}{|c|c|c|c|}
\hline $\begin{array}{l}\text { Price JR, Mitchell E, Tidy E, Hunot } \\
\text { V. Cognitive behaviour therapy for } \\
\text { chronic fatigue syndrome in adults. } \\
\text { Cochrane Database of } \\
\text { Systematic Reviews } 2008 \text {, Issue } 3 \text {. } \\
\text { Art. No.: CD001027. } \\
\text { DOI: } \\
\text { 10.1002/14651858.CD00102 } \\
\text { 7.pub2 - See more at: } \\
\text { http://summaries.cochrane.or } \\
\text { g/CD001027/DEPRESSN_co } \\
\text { gnitive-behaviour-therapyfor- } \\
\text { chronic- } \\
\text { fatiguesyndrome\#sthash.eLgdVIPR. } \\
\text { dpuf }\end{array}$ & $\begin{array}{l}\text { CBT for chronic } \\
\text { fatigue }\end{array}$ & $\begin{array}{l}\text { CBT is effective in } \\
\text { reducing the } \\
\text { symptoms of fatigue } \\
\text { at post-treatment } \\
\text { compared with } \\
\text { usual care, and may } \\
\text { be more effective in } \\
\text { reducing fatigue } \\
\text { symptoms compared } \\
\text { with other } \\
\text { psychological } \\
\text { therapies. The } \\
\text { evidence base at } \\
\text { follow-up is limited } \\
\text { to a small group of } \\
\text { studies with } \\
\text { inconsistent } \\
\text { findings. There is a } \\
\text { lack of evidence on } \\
\text { the }\end{array}$ & $\begin{array}{l}\text { Fifteen studies } \\
\text { (1043 CFS } \\
\text { participants) were } \\
\text { included in the } \\
\text { review. Randomised } \\
\text { controlled trials } \\
\text { involving adults } \\
\text { with a primary } \\
\text { diagnosis of CFS, } \\
\text { assigned to a CBT } \\
\text { condition compared } \\
\text { with usual care or } \\
\text { another } \\
\text { intervention, alone } \\
\text { or in combination. }\end{array}$ \\
\hline & & $\begin{array}{l}\text { comparative } \\
\text { effectiveness of } \\
\text { CBT alone or in } \\
\text { combination with } \\
\text { other treatments, } \\
\text { and further studies } \\
\text { are required to } \\
\text { inform the } \\
\text { development of } \\
\text { effective treatment } \\
\text { programmes for } \\
\text { people with CFS. }\end{array}$ & \\
\hline
\end{tabular}


Appendix B.

\section{Herbs used for Pain and Related Conditions in Appalachia: Anecdotal Sources/Herbal Texts}

Sources: Manual Search conducted March-April 2015 through West Virginia University Library Service (including databases PubMed, AltmedWatch, Library of Congress), WV Public Library search,

Google Search (with additional cross-referencing in all materials yielding items of interest to Appalachian herbs, botanicals, folk remedies, folk traditions, flora, non-timber plant products) with no publishing time range; Physical sources manually searched for 'Rheumatism;' 'Analgesia;' 'Pain;'

'Arthritis;' 'Inflammation;' 'Gout;' and, 'Headache'

\begin{tabular}{|c|c|c|c|}
\hline & \multicolumn{3}{|c|}{$* * * *$ Potential or known Narcotic - exercise caution } \\
\hline & \multicolumn{2}{|c|}{$* * *$ potential toxicity } & \\
\hline & \multicolumn{2}{|c|}{$\begin{array}{l}* * \text { known toxicity but not mentioned in this } \\
\text { literature }\end{array}$} & $\begin{array}{l}\text { Red lettering indicates use for } \\
\text { Inflammation }\end{array}$ \\
\hline & \multicolumn{2}{|c|}{ *known toxicity } & $\begin{array}{l}\text { YELLOW indicates a replicate } \\
\text { herb }\end{array}$ \\
\hline$\frac{\text { Herb identified in }}{\underline{\text { Appalachian Text }}}$ & $\frac{\frac{\text { Parts }}{\text { Used/Prepation }}}{\underline{\text { Method(s) }}}$ & Known for: & Source \\
\hline $\begin{array}{l}\text { Alfalfa (Medicago } \\
\text { sativa) }\end{array}$ & tops & Rheumatism & $\begin{array}{l}\text { Crellin, J. K., et al. (1990). } \\
\text { Herbal medicine past and } \\
\text { present. Durham, Duke } \\
\text { University Press. }\end{array}$ \\
\hline $\begin{array}{l}\text { Angelico (a. } \\
\text { atropurpurea) }\end{array}$ & $\begin{array}{l}\text { Tonic, roots and } \\
\text { leaves }\end{array}$ & $\begin{array}{l}\text { Chronic } \\
\text { Rheumatism }\end{array}$ & $\begin{array}{l}\text { Crellin, J. K., et al. (1997). } \\
\text { Trying to give ease : } \\
\text { Tommie Bass and the } \\
\text { story of herbal medicine. } \\
\text { \& Crellin, J. K., et al. } \\
\text { (1990). Herbal medicine } \\
\text { past and present. } \\
\text { Durham, Duke } \\
\text { University Press. } \\
\text { Durham, Duke University } \\
\text { Press. }\end{array}$ \\
\hline Apple & $\begin{array}{l}\text { Tea, syrup, and } \\
\text { Tonic of Bark or } \\
\text { fresh leaves }\end{array}$ & $\begin{array}{l}\text { Rheumatism; } \\
\text { arthritis }\end{array}$ & Crellin, J. K., et al. (1997). \\
\hline
\end{tabular}




\begin{tabular}{|c|c|c|c|}
\hline \multirow[b]{2}{*}{$\begin{array}{l}\text { Balm of Gilead } \\
\text { Balm of Gilead sap }\end{array}$} & $\begin{array}{l}\text { (not given) } \\
\text { (contains } \\
\text { crystalline } \\
\text { populus, closely } \\
\text { resembling } \\
\text { salicin) }\end{array}$ & Rheumatism & $\begin{array}{l}\text { Amjad, H. (2006). Folk } \\
\text { Medicine of } \\
\text { Appalachia: A Vanishing } \\
\text { Tradition. , lulu.com } \\
\text { (selfpublished). }\end{array}$ \\
\hline & Buds boiled in oil & Pain; salves & $\begin{array}{l}\text { Shelton, F. (1965). } \\
\text { Pioneer comforts and } \\
\text { kitchen remedies;: } \\
\text { Oldtimey highland } \\
\text { secrets from the Blue } \\
\text { Ridge and Great Smoky } \\
\text { Mountains, Hutcraft. }\end{array}$ \\
\hline $\begin{array}{l}\text { Balm of Gilead/Balsam } \\
\text { poplar/balm } \\
\text { buds/Carolina } \\
\text { poplar/cottonwood/hac } \\
\text { kmatack/poplar } \\
\text { balsam/tacamahac } \\
\text { poplar/tackamahac } \\
\text { (Salicaea) }\end{array}$ & $\begin{array}{l}\text { Tincture from } \\
\text { Winter buds in } \\
\text { February and } \\
\text { March, before } \\
\text { opening }\end{array}$ & Rheumatism;Gout & $\begin{array}{l}\text { Krochmal, A., et al. } \\
\text { (1971). A guide to } \\
\text { medicinal plants of } \\
\text { Appalachia. Washington,, } \\
\text { U.S. Forest Service; for } \\
\text { sale by the Supt. of Docs. }\end{array}$ \\
\hline $\begin{array}{l}\text { Balm of Gilead/Bam } \\
\text { a Gilly (Populus } \\
\text { balsamifera, } P \text {. } \\
\text { candicans) }\end{array}$ & $\begin{array}{l}\text { Salve, from } \\
\text { buds;infusion }\end{array}$ & $\begin{array}{l}\text { Rheumatism; } \\
\text { Strengthen nerves }\end{array}$ & $\begin{array}{l}\text { Bolyard, J. L. (1981). } \\
\text { Medicinal plants and } \\
\text { home remedies of } \\
\text { Appalachia. Springfield, } \\
\text { IIl., Thomas. }\end{array}$ \\
\hline
\end{tabular}




\begin{tabular}{|c|c|c|c|}
\hline $\begin{array}{l}\text { Balmony/White } \\
\text { turtlehead/Bitter } \\
\text { herb/Fishmouth/Saltrheum } \\
\text { weed/Shellflower/smo } \\
\text { oth } \\
\text { snakehead/snakehead/ } \\
\text { snakemouth/true } \\
\text { snakehead/turle } \\
\text { bloom/turle head } \\
\text { (Scrophulariaceae) }\end{array}$ & $\begin{array}{l} \\
\text { Herb, gathered } \\
\text { when flowering; } \\
\text { leaves, Gathered } \\
\text { in Spring }\end{array}$ & $\begin{array}{l}\text { Reducing } \\
\text { inflammation }\end{array}$ & $\begin{array}{l}\text { Krochmal, A., et al. } \\
\text { (1971). A guide to } \\
\text { medicinal plants of } \\
\text { Appalachia. } \\
\text { Washington,, U.S. } \\
\text { Forest Service; for sale } \\
\text { by the Supt. of Docs. }\end{array}$ \\
\hline Bay (magnolia) & Tonic & Rheumatism, sleep & $\begin{array}{l}\text { Crellin, J. K., et al. } \\
\text { (1997). Trying to give } \\
\text { ease: }\end{array}$ \\
\hline Bearsfoot & $\begin{array}{l}\text { Salve, Root and } \\
\text { leaves }\end{array}$ & $\begin{array}{l}\text { Rheumatism; } \\
\text { arthritis }\end{array}$ & $\begin{array}{l}\text { Crellin, J. K., et al. } \\
\text { (1990). H }\end{array}$ \\
\hline Beech & Tonic & Rheumatism & $\begin{array}{l}\text { Crellin, J. K., et al. (1997) } \\
T \text { T }\end{array}$ \\
\hline Bergamot/White horsem & $\begin{array}{l}\text { infusion, from } \\
\text { leav }\end{array}$ & Rheumatism & $\begin{array}{l}\text { Bolyard, J. L. (1981). } \\
\text { Medi }\end{array}$ \\
\hline Bethroot & Tea, Rhizome & $\begin{array}{l}\text { Rheumatism; } \\
\text { headache }\end{array}$ & $\begin{array}{l}\text { Crellin, J. K., et al. } \\
\text { (1990). H }\end{array}$ \\
\hline Birch (B. lenta) & leaves, & (Rheumatism; Gout & $\begin{array}{l}\text { Crellin, J. K., et al. } \\
(1990) . \text { H }\end{array}$ \\
\hline Black Birch & Sap (in salve) & Rheu; massage & $\begin{array}{l}\text { Shelton, F. (1965). } \\
\text { Pionee }\end{array}$ \\
\hline $\begin{array}{l}\text { Black CohoshBlack } \\
\text { Cohosh/Black }\end{array}$ & Tonic, roots & Rheumatism & $\begin{array}{l}\text { Crellin, J. K., et al. (1997) } \\
T\end{array}$ \\
\hline $\begin{array}{l}\text { snakeroot/Rattleweed/ } \\
\text { Squawweed/Cocash } \\
\text { (Indian name)/Bugbane }\end{array}$ & $\begin{array}{l}\text { Tincture, } \\
\text { Poultice or tea, } \\
\text { of boiled root }\end{array}$ & $\begin{array}{l}\text { Rheumatism; } \\
\text { soothe pain }\end{array}$ & $\begin{array}{l}\text { Bolyard, J. L. (1981). } \\
\text { Medi }\end{array}$ \\
\hline Black Haw/Crampbark & Bark & Rheumatism & $\begin{array}{l}\text { Crellin, J. K., et al. } \\
\text { (1990). H }\end{array}$ \\
\hline Black Snakeroot & Root & Rheumatism & $\begin{array}{l}\text { Crellin, J. K., et al. } \\
\text { (1990). H }\end{array}$ \\
\hline $\begin{array}{l}\text { Blue cohosh/Blueberry } \\
\text { cohosh/blueberry } \\
\text { root/papoose root/ Sqaw } \\
\text { root/ Yellow ginseng } \\
\text { (Caulophyllum } \\
\text { thalictroides L.) }\end{array}$ & $\begin{array}{l}\text { Rhizomes and } \\
\text { roots, gathered } \\
\text { in fall }\end{array}$ & $\begin{array}{l}\text { Chronic } \\
\text { rheumatism }\end{array}$ & $\begin{array}{l}\text { Krochmal, A., et al. } \\
\text { (1971). }\end{array}$ \\
\hline
\end{tabular}


Blue vervain/blue

verbena/ironweed/

simpler's joy/verbain/wild

hyssop (Verbenaceae)

Herb and root Antirheumatic

Krochmal, A., et al.

(1971). 


\begin{tabular}{|c|c|c|c|}
\hline Boneset & $\begin{array}{l}\text { Tea, Leaves and } \\
\text { roots }\end{array}$ & $\begin{array}{l}\text { Rheumatism; } \\
\text { headache }\end{array}$ & Crellin, J. K., et al. (1997). \\
\hline $\begin{array}{l}\text { Boneset/Thoroughwort } \\
\text { /Feverwort/Indian } \\
\text { Sage/Agueweed } \\
\text { (Eupatorium } \\
\text { perfoliatum) }\end{array}$ & $\begin{array}{l}\text { Decoction of roots } \\
\text { and leaves }\end{array}$ & Rheumatism & Bolyard, J. L. (1981). Medi \\
\hline $\begin{array}{l}\text { Bowman's } \\
\text { Root/ipecac/Indian } \\
\text { physic }\end{array}$ & Tonic, bark & Rheumatism & Crellin, J. K., et al. (1997). \\
\hline Buckeye & Fruit & Rheumatism & $\begin{array}{l}\text { Wilson, C. R. and W. R } \\
\text { Ferr }\end{array}$ \\
\hline Buckeye & $\begin{array}{l}\text { Salve, Seeds } \\
\text { (nonpoisonous } \\
\text { half) and bark }\end{array}$ & Rheumatism & $\begin{array}{l}\text { Crellin, J. K., et al. (1990). } \\
\text { H }\end{array}$ \\
\hline $\begin{array}{l}\text { Buckeye* (Aesculus } \\
\text { glabra, A. octandra) }\end{array}$ & Fruit & $\begin{array}{l}\text { Rheumatism; used } \\
\text { in place of opium } \\
\text { ( } 10 \text { grains } \\
\text { powdered buckeye } \\
\text { fruit rind = } 3 \text { grains } \\
\text { opium) }\end{array}$ & Bolyard, J. L. (1981). Medi \\
\hline Burdock & Roots, seeds & Rheumatism & $\begin{array}{l}\text { Crellin, J. K., et al. (1990). } \\
\text { H }\end{array}$ \\
\hline Burdock & Tea, roots \& seeds & Rheumatism & Shelton, F. (1965). Pionee \\
\hline $\begin{array}{l}\text { Burdock/Great Burdock } \\
\text { (Arctium lappa) }\end{array}$ & seed and roots & $\begin{array}{l}\text { Rheumatism;Gout; } \\
\text { Inflammations }\end{array}$ & Bolyard, J. L. (1981). Medi \\
\hline Butterfly Weed & Tea, Root & $\begin{array}{l}\text { Rheumatism; } \\
\text { Anodyne }\end{array}$ & $\begin{array}{l}\text { Crellin, J. K., et al. (1990). } \\
\text { H }\end{array}$ \\
\hline Button snakeroot & Root & Rheumatism; Gout & Crellin, J. K., et al. (1997). \\
\hline Catnip (Nepeta cataria) & Tea & $\begin{array}{l}\text { Sleep Aid, Nervous } \\
\text { Depression, } \\
\text { Debility }\end{array}$ & $\begin{array}{l}\text { Amjad, H. (2006). Foll } \\
\text { Med }\end{array}$ \\
\hline Cedar & Oil, tops, "apples" & $\begin{array}{l}\text { Chronic } \\
\text { Rheumatism }\end{array}$ & $\begin{array}{l}\text { Crellin, J. K., et al. (1990). } \\
H\end{array}$ \\
\hline Celery; Smallage (wild) & fruits, roots, tops & $\begin{array}{l}\text { Rheumatism; } \\
\text { Inflammation; } \\
\text { migraine }\end{array}$ & $\begin{array}{l}\text { Crellin, J. K., et al. (1990). } \\
\text { H }\end{array}$ \\
\hline Chamomile & flowers & $\begin{array}{l}\text { Aches, pains, } \\
\text { bruises }\end{array}$ & $\begin{array}{l}\text { Crellin, J. K., et al. (1990). } \\
\text { H }\end{array}$ \\
\hline
\end{tabular}




\begin{tabular}{|c|c|c|c|}
\hline $\begin{array}{l}\text { Chaparral/Creosote } \\
\text { bush*** }\end{array}$ & $\begin{array}{l}\text { Tea and external, } \\
\text { leaves }\end{array}$ & Rheumatism & Crellin, J. K., et al. (1990). - \\
\hline $\begin{array}{l}\text { Chestnut/American } \\
\text { Chestnut (Fagaceae) }\end{array}$ & $\begin{array}{l}\text { tea from leaves } \\
\text { and inner bark }\end{array}$ & $\begin{array}{l}\text { Rheumatism; } \\
\text { arthritis }\end{array}$ & Bolyard, J. L. (1981). Medi \\
\hline Chicory & Tea, of leaves & Rheu; stomach & Shelton, F. (1965). Pionee \\
\hline $\begin{array}{l}\text { Club } \\
\text { Moss/Moss/Running } \\
\text { Cedar }\end{array}$ & Tops & Rheumatism & Crellin, J. K., et al. (1990). - \\
\hline $\begin{array}{l}\text { Cohoshes (black, blue, } \\
\text { white) }\end{array}$ & $\begin{array}{l}\text { Rhizome, roots } \\
\text { (beat up the root) }\end{array}$ & $\begin{array}{l}\text { Rheumatism, } \\
\text { soothe pain, allay } \\
\text { irritability, possible } \\
\text { anti-inflammatory }\end{array}$ & Crellin, J. K., et al. (1990). I \\
\hline Colic Root (Aletris) & $\begin{array}{l}\text { Roots gathered in } \\
\text { fall }\end{array}$ & Rheumatism & Collins, K. C. and L. Hunter \\
\hline Collards & $\begin{array}{l}\text { Roots, applied } \\
\text { externally }\end{array}$ & $\begin{array}{l}\text { Arthrtitic Joints for } \\
\text { pain }\end{array}$ & $\begin{array}{l}\text { Brunvand, J. H. (1996). } \\
\text { American folklore : an } \\
\text { encyclopedia. New York, } \\
\text { Garland Pub. }\end{array}$ \\
\hline Corn Silk & Styles, stigmas & Rheumatism; Gout & Crellin, J. K., et al. (1990). I \\
\hline Cottonwood Tree & $\begin{array}{l}\text { in liniment with } \\
\text { turpentine, Bark, } \\
\text { leaves, green fruit }\end{array}$ & Rheumatism & Crellin, J. K., et al. (1990). $\vdash$ \\
\hline $\begin{array}{l}\text { Cucumber tree } \\
\text { (magnolia) }\end{array}$ & Bark & $\begin{array}{l}\text { Rheumatism } \\
\text { (claimed "the best") }\end{array}$ & Crellin, J. K., et al. (1997). T \\
\hline $\begin{array}{l}\text { Cucumber } \\
\text { tree/Wahoo/Cucumber } \\
\text { magnolia } \\
\text { (Magnoliaceae) }\end{array}$ & Bark & Rheumatism & Bolyard, J. L. (1981). Medi \\
\hline $\begin{array}{l}\text { Dandelion (Taraxacum } \\
\text { officinale) }\end{array}$ & $\begin{array}{l}\text { decoction, roots } \\
\text { and leaves }\end{array}$ & $\begin{array}{l}\text { Rheumatism; } \\
\text { bursitis;arthritis }\end{array}$ & Bolyard, J. L. (1981). Medi \\
\hline $\begin{array}{l}\text { Devil's } \\
\text { shoestring/Goat's Rue }\end{array}$ & Tea, Root & Rheumatism & Crellin, J. K., et al. (1997). T \\
\hline
\end{tabular}




\begin{tabular}{|l|l|l|l|}
\hline $\begin{array}{l}\text { Echinacea/Purple } \\
\text { Coneflower (Echinacea) }\end{array}$ & $\begin{array}{l}\text { Extracts, of roots } \\
\text { gathered in the }\end{array}$ & $\begin{array}{l}\text { Chronic } \\
\text { fall }\end{array}$ & Collins, K. C. and L. Hunter \\
\hline $\begin{array}{l}\text { Elderberry (Sambucus Flowers and juice } \\
\text { canadensis) } \\
\text { of berries }\end{array}$ & Rheumatism & Amjad, H. (2006). Folk Mec \\
$\begin{array}{l}\text { Elderberry/Common } \\
\text { elder (Sambucus } \\
\text { canadensis) }\end{array}$ & Berries & Rheumatismic gout & Bolyard, J. L. (1981). Medi \\
\hline & & & \\
\hline
\end{tabular}




\begin{tabular}{|c|c|c|c|}
\hline Garlic & Tea, fresh roots & Rheumatism & Shelton, F. (1965). Pionee \\
\hline Gentian & Root & Rheumatism & Crellin, J. K., et al. (1997). 1 \\
\hline $\begin{array}{l}\text { Ginger (Zingiber } \\
\text { officinale) }\end{array}$ & (not given) & Rheumatic pain & $\begin{array}{l}\text { Amjad, H. (2006). Foll } \\
\text { Med }\end{array}$ \\
\hline Ginseng & Roots & Rheumatism? & $\begin{array}{l}\text { Crellin, J. K., et al. (1990). } \\
\text { H }\end{array}$ \\
\hline Ginseng & Roots, chewed & $\begin{array}{l}\text { Arthritis/ } \\
\text { Rheumatism }\end{array}$ & $\begin{array}{l}\text { Goodwin, J. G. (1985) } \\
\text { Goin }\end{array}$ \\
\hline $\begin{array}{l}\text { Ginseng } \\
\text { quinquefolia ) }\end{array}$ & $\begin{array}{l}\text { Tea, of roots } \\
\text { gathered in fall }\end{array}$ & $\begin{array}{l}\text { Arthritis; calming } \\
\text { effect on nerves }\end{array}$ & $\begin{array}{l}\text { Amjad, H. (2006). Foll } \\
\text { Med }\end{array}$ \\
\hline $\begin{array}{l}\text { Ginseng/Seng/Sang/Tar } \\
\text { tar } \\
\text { root/Fivefinger/Redber } \\
\text { ry/Garantoqueen (Panax } \\
\text { quinquefolium) }\end{array}$ & Tea, from root & $\begin{array}{l}\text { Rheumatism; } \\
\text { arthritis;bursitis }\end{array}$ & Bolyard, J. L. (1981). Medi \\
\hline $\begin{array}{l}\text { Goldenseal/Yellowroot } \\
\text { (Hydrastis canadensis ) }\end{array}$ & Tea, from root & $\begin{array}{l}\text { Rheumatism; } \\
\text { arthritis;bursitis }\end{array}$ & Bolyard, J. L. (1981). Medi \\
\hline Gotu Kola & Leaves, whole herb & Rheumatism & $\begin{array}{l}\text { Crellin, J. K., et al. (1990). } \\
\text { H }\end{array}$ \\
\hline Ground Ivy & $\begin{array}{l}\text { Tea, from Leaves } \\
\text { (green or dry) }\end{array}$ & Headache & $\begin{array}{l}\text { Crellin, J. K., et al. (1990). } \\
\text { H }\end{array}$ \\
\hline $\begin{array}{l}\text { Holly/American } \\
\text { Holly/Huver Bush } \\
\text { (Aquifoliaceae) }\end{array}$ & Decoction of bark & In place of opium & Bolyard, J. L. (1981). Medi \\
\hline Hops (Humulus lupulus ) & Poultice & $\begin{array}{l}\text { Inflammatory } \\
\text { conditions }\end{array}$ & $\begin{array}{l}\text { Amjad, H. (2006). Foll } \\
\text { Med }\end{array}$ \\
\hline $\begin{array}{l}\text { Horehound } \\
\text { (Marrubium vulgare) }\end{array}$ & (not given) & Rheumatism & $\begin{array}{l}\text { Amjad, H. (2006). Foll } \\
\text { Med }\end{array}$ \\
\hline $\begin{array}{l}\text { Horse } \\
\text { Chestnut/Buckeye } \\
\text { Tree/Common } \\
\text { horsechestnut (Aesculus } \\
\text { hippocastanum L.) }\end{array}$ & $\begin{array}{l}\text { Bark and fruit, } \\
\text { gathered in fall } \\
\text { (carried around) }\end{array}$ & Rheumatism & $\begin{array}{l}\text { Krochmal, A., et al. } \\
\text { (1971). }\end{array}$ \\
\hline
\end{tabular}




\begin{tabular}{|c|c|c|c|}
\hline $\begin{array}{l}\text { Horseradish/Scurvy } \\
\text { Grass }\end{array}$ & $\begin{array}{l}\text { Poultice, Roots and } \\
\text { Leaves (used } \\
\text { as } \\
\text { counterirritant)bark, } \\
\text { leaves, and }\end{array}$ & $\begin{array}{l}\text { Rheumatism and } \\
\text { paralytic complaints }\end{array}$ & $\begin{array}{l}\text { Crellin, J. K., et al. (1990). } \\
\text { H }\end{array}$ \\
\hline Hydrangea & root & Rheumatism & Crellin, J. K., et al. (1997). 7 \\
\hline Indian hemp/Dogbane & Root & Rheumatism & Crellin, J. K., et al. (1997). 7 \\
\hline $\begin{array}{l}\text { Indian hemp/North- } \\
\text { andsouth root } \\
\text { (Apocynum cannabinum) }\end{array}$ & $\begin{array}{l}\text { Decoction of Root, } \\
\text { gathered in fall }\end{array}$ & $\begin{array}{l}\text { Rheumatism; } \\
\text { arthritis;bursitis; } \\
\text { migraine }\end{array}$ & Bolyard, J. L. (1981). Medi \\
\hline & Poultice, of dried & & \\
\hline
\end{tabular}


Jimsonweed*/apple of peru/apple peru/devil's trumpet/Jamestown weed/jimsonweed datura/mad apple/stink apple/stinkweed/stink wort/stramonium/thor $\mathrm{n}$ apple (Solanaceae)

Joe-Pye-Weed

(Eupatorium sp. )

Lady-slipper/Moccasin

flower/Nerve root

(Cypripedium spp.)

\begin{tabular}{|c|c|c|c|}
\hline & & & \\
\hline Lemon & $\begin{array}{l}\text { fruit, peel, volatile } \\
\text { oil }\end{array}$ & $\begin{array}{l}\text { Rheumatism Anti- } \\
\text { inflammatory; }\end{array}$ & $\begin{array}{l}\text { Crellin, J. K., et al. (1990). } \\
\text { H }\end{array}$ \\
\hline Licorice & Rhizome, roots & Rheumatism & $\begin{array}{l}\text { Crellin, J. K., et al. (1990). } \\
\text { H }\end{array}$ \\
\hline $\begin{array}{l}\text { Maidenhair Fern } \\
\text { (Adiantum pedatum ) }\end{array}$ & Roots & Rheumatism & Collins, K. C. and L. Hunter \\
\hline $\begin{array}{l}\text { Mayapple****/ Mandrake } \\
\text { (Berberidaceae) }\end{array}$ & Decotion, of Root & $\begin{array}{l}\text { Rheumatism; } \\
\text { bursitis;arthritis }\end{array}$ & Bolyard, J. L. (1981). Medi \\
\hline $\begin{array}{l}\text { Milkweed/Common } \\
\text { milkweed/Common } \\
\text { silkweed/cottonweed/s } \\
\text { ilkweed/swallowwort/wild } \\
\text { cotton (Asclepiadaceae) }\end{array}$ & $\begin{array}{l}\text { Roots, gathered in } \\
\text { fall }\end{array}$ & Rheumatism & $\begin{array}{l}\text { Krochmal, A., et al. } \\
\text { (1971). }\end{array}$ \\
\hline $\begin{array}{l}\text { Milkweed/cottonweed/ } \\
\text { silkweed/wild cotton } \\
\text { (Asclepiadaceae) }\end{array}$ & & $\begin{array}{l}\text { Inflammatory } \\
\text { Rheumatism }\end{array}$ & Bolyard, J. L. (1981). Medi \\
\hline
\end{tabular}

Leaves and tops when flowering, Anodyne seeds when sedative/Antispasm Krochmal, A., et al. mature odic (1971).

Crellin, J. K., et al. (1997). 1

Sedative;

Antispasmodic;

Allays pain

Bolyard, J. L. (1981). Medi

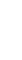


Moonseed/common moonseed/canada

moonseed/maple

vine/Texas

sarsaparilla/vine

maple/yellow

Rhizomes and

parilla/yellow sarsaparilla roots, gathered in

(Menispermaceae)

fall

Arthritic conditions

Krochmal, A., et al.

Mountain

Tea/Checkerberry/Teab

erry (Gautheria

Muscle

ache/Rheumatism

(1971). 
Pennyroyal/pennyrile/

Pudding grass/Squaw

mint/Stinking

balm/Tickweed

(Hedeoma pulegioides )

fresh or dried

leaves

(Hedeoma pulegioides)

Peppermint (Mentha fresh leaves

piperita )

Pine

Pine
Pipsissewa/Common
pipsissewa/Bitter
wintergreen/fragrant
wintergreen/ground
holly/king's cure/lovein-
winter/noble pine/Pine
tulip/prince's
pine/princess
pine/pyrole/rheumatis
m weed/

waxflower/wintergreen

(Pyrolaceae)

Pipsissewa/Spidercheck

ered

wintergreen/Striped

wintergreen

Pleurisy Root (Asclepias

tuberosa)

Pleurisy Root/Butterfly

Weed (Asclepias

tuberosa)

Poke

Poke

americana )*

Poke*/Pokeweed/Poke root/Poke salat/Poke

\begin{tabular}{|c|c|c|}
\hline $\begin{array}{l}\text { fresh or dried } \\
\text { leaves }\end{array}$ & $\begin{array}{l}\text { Headache; } \\
\text { Rheumatism }\end{array}$ & Bolyard, J. L. (1981). Medi \\
\hline $\begin{array}{l}\text { fresh leaves } \\
\text { applied externally }\end{array}$ & $\begin{array}{l}\text { headache; slight } \\
\text { anesthetic; local } \\
\text { pain }\end{array}$ & Bolyard, J. L. (1981). Medi \\
\hline Sap and bark oil & Massage; stiffness & Shelton, F. (1965). Pionee \\
\hline $\begin{array}{l}\text { tops, bark, sap, } \\
\text { tar, turpentine, } \\
\text { rosin }\end{array}$ & Rheumatism & Crellin, J. K., et al. (1990). \\
\hline $\begin{array}{l}\text { Leaves and herbs, } \\
\text { gathered in late } \\
\text { summer or early } \\
\text { fall }\end{array}$ & Anti-rheumatic & Krochmal, A., et al. (1971). \\
\hline (not given) & $\begin{array}{l}\text { Arthritis/Rheumatis } \\
\mathrm{m} / \text { Bursitis }\end{array}$ & Bennett, Allen and Green, \\
\hline Tea, of roots & Rheumatism & Amjad, H. (2006). Folk Mec \\
\hline $\begin{array}{l}\text { Tea, of roots } \\
\text { gathered in fall }\end{array}$ & Aching muscles & Collins, K. C. and L. Hunter \\
\hline $\begin{array}{l}\text { Internal and } \\
\text { external, Root } \\
\text { and berries }\end{array}$ & Rheumatism & Crellin, J. K., et al. (1997). T \\
\hline $\begin{array}{l}\text { Ash-roasted root } \\
\text { applied externally }\end{array}$ & Inflamed joints & Collins, K. C. and L. Hunter \\
\hline
\end{tabular}


beryy/Jalap/Cancerroot

/Scoke/Garget

Tea or tincture of

berries; decoction Rheumatism;arthrit 


\begin{tabular}{|c|c|c|c|}
\hline Pokeweed* & $\begin{array}{l}\text { Berries (small } \\
\text { quantity) }\end{array}$ & Rheumatism & $\begin{array}{l}\text { Amjad, H. (2006). Foll } \\
\text { Med }\end{array}$ \\
\hline $\begin{array}{l}\text { Pokeweed*/American } \\
\text { nightshade/cancer } \\
\text { jalap/cancerroot/chong } \\
\text { ras/coakum/cocum/cok } \\
\text { an/common } \\
\text { pokeberry/crowberry/g } \\
\text { arget/ } \\
\text { inkberry/jalap/pigeonb } \\
\text { erry/ pocan/pocan } \\
\text { bush/poke/pokeberry/ } \\
\text { pokeroot/red-ink } \\
\text { plant/red } \\
\text { wood/scoke/skoke/Virg } \\
\text { inia poke }\end{array}$ & $\begin{array}{l}\text { le } \\
\\
\\
\text { Root, gathered } \\
\text { in fall; ripe } \\
\text { berries made } \\
\text { into wine }\end{array}$ & Rheumatism & $\begin{array}{l}\text { Krochmal, A., et al. } \\
\text { (1971). }\end{array}$ \\
\hline $\begin{array}{l}\text { Poplars (including } \\
\text { Yellow) }\end{array}$ & $\begin{array}{l}\text { Tonic, from bark, } \\
\text { root bark, and } \\
\text { salves from buds }\end{array}$ & Rheumatism & $\begin{array}{l}\text { Crellin, J. K., et al. (1990). } \\
\text { H }\end{array}$ \\
\hline Prickly Ash Prickly Ash & $\begin{array}{l}\text { Chewed tree bark } \\
\text { (not root) and tea } \\
\text { from berries }\end{array}$ & Rheumatism & Crellin, J. K., et al. (1997). \\
\hline $\begin{array}{l}\text { (Xanthoxylum } \\
\text { americanum) }\end{array}$ & $\begin{array}{l}\text { Syrup of berries } \\
\text { and bark }\end{array}$ & Rheumatism & $\begin{array}{l}\text { Amjad, H. (2006). Foll } \\
\text { Med }\end{array}$ \\
\hline Prickly Pear & fruit, fleshy leaves & Acute rheumatism & $\begin{array}{l}\text { Crellin, J. K., et al. (1990). } \\
\text { H }\end{array}$ \\
\hline $\begin{array}{l}\text { Pussy Willow/Possum } \\
\text { bush/Swamp willow } \\
\text { (Salix spp.) }\end{array}$ & $\begin{array}{l}\text { tea, from limbs or } \\
\text { leaves }\end{array}$ & $\begin{array}{l}\text { Headache; Pain; } \\
\text { Gout }\end{array}$ & Bolyard, J. L. (1981). Medi \\
\hline Quaking Aspen & Tea, of bark & Muscle pain & Shelton, F. (1965). Pionee \\
\hline Queen Anne's Lace & Tops and roots & Rheumatism & Crellin, J. K., et al. (1997). \\
\hline 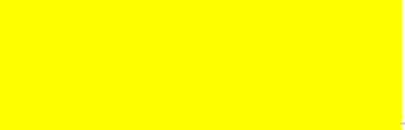 & minute quantities & Rheumatism; gout & $\begin{array}{l}\text { Amjad, H. (2006). Foll } \\
\text { Med }\end{array}$ \\
\hline $\begin{array}{l}\text { Ratsbane/Quaker's } \\
\text { buttons (Nux vomica)* } \\
\text { Ratsvane/Pipsissewa }\end{array}$ & $\begin{array}{l}\text { Leaves, roots, } \\
\text { whole plant }\end{array}$ & Rheumatism & Crellin, J. K., et al. (1997). \\
\hline
\end{tabular}




\begin{tabular}{|c|c|c|c|}
\hline $\begin{array}{l}\text { Redroot****/Blodroot/ } \\
\text { puccoon } \\
\text { (Papaveraceae) }\end{array}$ & tonic or bitters & $\begin{array}{l}\text { Rheumatism;Gout; } \\
\text { Arthritis }\end{array}$ & $\begin{array}{l}\text { Krochmal, A., et al. } \\
\text { (1971). }\end{array}$ \\
\hline $\begin{array}{l}\text { Redroot*/Blodroot/coo } \\
\text { nroot/pauson/puccoon/ } \\
\text { puccoon root/red } \\
\text { Indian paint/red } \\
\text { puccoon/snakebite/sw } \\
\text { eet } \\
\text { slumber/tetterwort/tur } \\
\text { meric/white puccoon } \\
\text { (Papaveraceae) }\end{array}$ & $\begin{array}{l}\text { Rootstock when } \\
\text { flowering, in late } \\
\text { summer or early } \\
\text { fall }\end{array}$ & $\begin{array}{l}\text { pain } \\
\text { reliever;sedative }\end{array}$ & $\begin{array}{l}\text { Krochmal, A., et al. } \\
\text { (1971). }\end{array}$ \\
\hline Sampson snakeroot & $\begin{array}{l}\text { Tea, Tonic and } \\
\text { root }\end{array}$ & Rheumatism & $\begin{array}{l}\text { Crellin, J. K., et al. (1997) } \\
T \text { T }\end{array}$ \\
\hline $\begin{array}{l}\text { Sampson's } \\
\text { snakeroot/marsh } \\
\text { gentian/straw-colored } \\
\text { gentian/striped gentian } \\
\text { (Gentianaceae) }\end{array}$ & $\begin{array}{l}\text { Tonic of Roots, } \\
\text { gathered in fall }\end{array}$ & Rheumatism/Gout & $\begin{array}{l}\text { Krochmal, A., et al. } \\
\text { (1971). }\end{array}$ \\
\hline Sarsparilla & Rhizome, roots & Rheumatism & $\begin{array}{l}\text { Crellin, J. K., et al. (1990). } \\
\text { H }\end{array}$ \\
\hline $\begin{array}{l}\text { Sasparilla/Sasparillo/Sa } \\
\text { safarilla/Safarilla/Sasfril } \\
\text { ler/Sarsparilla/Moonse } \\
\text { ed (Menispermum } \\
\text { canadense) }\end{array}$ & $\begin{array}{l}\text { Tea or tonic, from } \\
\text { root }\end{array}$ & Rheumatism/Gout & Bolyard, J. L. (1981). Medi \\
\hline Sassafras & $\begin{array}{l}\text { Root bark, wood, } \\
\text { pith, leaves }\end{array}$ & Rheumatism & $\begin{array}{l}\text { Crellin, J. K., et al. (1997) } \\
\text { T }\end{array}$ \\
\hline Sassafras & $\begin{array}{l}\text { Tea (roots } \\
\text { and bark), oil } \\
\text { of sassafras }\end{array}$ & $\begin{array}{l}\text { Rheu; general } \\
\text { tonic/massage; pain }\end{array}$ & Shelton, F. (1965). Pionee \\
\hline
\end{tabular}


Sassafras/Sassafac/Sass

afrax (Sassafras

albidum)

Decoction from

root

Rheumatism

Bolyard, J. L. (1981). Medi

\section{Seneca}

snakeroot/Senneker

snakeroot/Sinnaker

snakeroot/Milkwort/Ra

ttlesnake Root (Polygala

senega )

Root

Rheumatism; gout Bolyard, J. L. (1981). Medi

Senega

Root

Crellin, J. K., et al. (1990).

Rheumatism H

bark

Arthritis/Rheumatis Crellin, J. K., et al. (1990).

Sevenbark

bark

m H

Shepherd's Purse

(Capsella bursapastoris)

Poultice

(n)

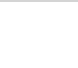

$\mathrm{H}$

Amjad, H. (2006). Foll

Tonic, Leaves, Rheumatism,

Skullcap

stem, whole plant nervous conditions

Crellin, J. K., et al. (1997).

Smartweed/Celery/Pars Salves and tea,

Crellin, J. K., et al. (1990).

ley

from Whole plant Rheumatism

$\mathrm{H}$

\begin{tabular}{|c|c|c|}
\hline Soapwort & $\begin{array}{l}\text { Tea, of } \\
\text { roots/external }\end{array}$ & $\begin{array}{l}\text { Rheumatism; } \\
\text { gout/Boils;skin } \\
\text { sores }\end{array}$ \\
\hline
\end{tabular}

\begin{tabular}{|c|c|c|c|}
\hline soapwort & roots/external & sores & Shelton, F. (1965). Pionee \\
\hline Solomon's Seal & Boiled root & Inflammation & $\begin{array}{l}\text { Amjad, H. (2006). Foll } \\
\text { Med }\end{array}$ \\
\hline $\begin{array}{l}\text { Spanish Moss } \\
\text { (Tillandsia usneiodes ) }\end{array}$ & (not given) & Rheumatism & $\begin{array}{l}\text { Amjad, H. (2006). Foll } \\
\text { Med }\end{array}$ \\
\hline $\begin{array}{l}\text { Spicebush } \quad \text { (Linderia } \\
\text { benzoin ) }\end{array}$ & Berries & Rheumatism & $\begin{array}{l}\text { Amjad, H. (2006). Foll } \\
\text { Med }\end{array}$ \\
\hline $\begin{array}{l}\text { Spicewood/Spicebush/ } \\
\text { Wild allspice (Linderia } \\
\text { benzoin) }\end{array}$ & $\begin{array}{l}\text { oil from fruit } \\
\text { applied externally }\end{array}$ & Rheumatism & Bolyard, J. L. (1981). Medi \\
\hline
\end{tabular}


Rheumatism/Rheu

m accompanied by Amjad, H. (2006)

Root

back pain/Arthritis Folk Med

Tea from roots,

gathered in

Spikenard (Aralia racemosa)

Summer and

Spikenard/American

fall Rheumatism

Krochmal, A., et al.

spikenard/American

sarsaparilla/Indian root/life-ofman/old man's root/petty morel/pigeon weed/spignet

(Araliaceae)

Spikenard/Spignet/Spik

enard/Goutwort/Petty

morrell/Indian root (Aralia

racemosa)

Squaw Root/Black Cohosh

Squaw root/cohosh

bugbane/battle weed/black

cohosh/black

snakeroot/blueberry/bl ue

ginseng/bugbane/coho sh/heart-

leaved rattletop/heart-leaved

snakeroot/meadow rue

leontice/papoose root/rattle

root/rattlesnake

Rhizomes and

root/rattletop/rattleweed/richwe roots,

ed/yellow ginseng

gathered

in Chronic

the fall

rheumatism

Krochmal, A., et al.

(Ranunculaceae)

\begin{tabular}{l|l|l|}
\hline Decoction & arthritis;backaches & Medi \\
\hline & & Amjad, H. (2006) \\
(not given) & Rheumatism & Folk Med
\end{tabular}

(1971).

Folk Med

(1971). 


\begin{tabular}{|c|c|c|c|}
\hline \multirow[b]{2}{*}{$\begin{array}{l}\text { Star Grass } \\
\text { Star-root/Stargrass } * * * *\end{array}$} & $\begin{array}{l}\text { sip (in alcohol), } \\
\text { roots }\end{array}$ & Rheumatism & Shelton, F. (1965). Pionee \\
\hline & Root & Rheumatism & $\begin{array}{l}\text { Crellin, J. K., et al. (1990). } \\
\text { H }\end{array}$ \\
\hline Swamp Root & (not given) & Rheumatism & $\begin{array}{l}\text { Amjad, H. (2006). Foll } \\
\text { Med }\end{array}$ \\
\hline Sweet Birch & $\begin{array}{l}\text { Roots, bark } \\
\text { (distilled) }\end{array}$ & Massage & Shelton, F. (1965). Pionee \\
\hline $\begin{array}{l}\text { Sweet } \\
\text { Birch/Black } \\
\text { birch/cherry } \\
\text { birch/mountain } \\
\text { mahogony/River } \\
\text { birch/Spice birch } \\
\text { (Corylaceae) }\end{array}$ & $\begin{array}{l}\text { Bark and twigs, } \\
\text { gathered May to } \\
\text { September }\end{array}$ & Antirheumatic & $\begin{array}{l}\text { Krochmal, A., et al. } \\
\text { (1971). }\end{array}$ \\
\hline $\begin{array}{l}\text { Sweet Birch/Black } \\
\text { Birch/Cherry } \\
\text { Birch/Spice } \\
\text { Birch/Mountain } \\
\text { Mahogony (Betulaceae) }\end{array}$ & $\begin{array}{l}\text { Twigs, cones, } \\
\text { bark, leaves, and } \\
\text { juice }\end{array}$ & $\begin{array}{l}\text { Pain relief; } \\
\text { antirheumatic }\end{array}$ & Bolyard, J. L. (1981). Medi \\
\hline $\begin{array}{l}\text { Sweet Fern (Comptonia } \\
\text { peregrina) }\end{array}$ & $\begin{array}{l}\text { Tea, of leaves and } \\
\text { tops }\end{array}$ & Rheumatism & Collins, K. C. and L. Hunter \\
\hline Tansy & Tea & Aches and Pains & \\
\hline $\begin{array}{l}\text { Tobacco (Nicotania } \\
\text { tabacum) }\end{array}$ & $\begin{array}{l}\text { Juice or ointment, } \\
\text { applied externally }\end{array}$ & Rheumatic pains & Bolyard, J. L. (1981). Medi \\
\hline $\begin{array}{l}\text { Veratrum/hellebore } \\
* * *\end{array}$ & $\begin{array}{l}\text { Salve, from } \\
\text { rhizome and } \\
\text { roots (much attn } \\
\text { given for many } \\
\text { alkoloids present } \\
\text { and their role in } \\
\text { muscle-relaxant } \\
\text { and } \\
\text { antihypertensive } \\
\text { actions -JF } \\
\text { Morton (1977, } \\
\text { pp.58-64) }\end{array}$ & Rheumatism; gout & $\begin{array}{l}\text { Crellin, J. K., et al. (1990). } \\
\text { H }\end{array}$ \\
\hline
\end{tabular}


Tea, or in wine or

whiskey, Rhizome

Virginia Snakeroot atercress/nasturtium/

true watercress

(Brassicaceae)

and roots

Arthritis

Crellin, J. K., et al. (1990).

Waxflower

(Ratsbane)**

White ash/American

Ash/American white

ash/biltmore

Inner bark of

ash/biltmore white trunk and roots,

ash/cane ash/smallseed stem, both in

white ash (Oleaceae)

Spring

Antiarthritic

Krochmal, A., et al.

White Ash/Ash

Rheumatism; gout;

(Fraxinus americana?) Bark, and leaves arthritis Bolyard, J. L. (1981). Medi

White Pine (Pinus Resin from inner

strobus)

bark, sprigs Rheumatism

Bolyard, J. L. (1981). Medi

\begin{tabular}{|l|l|l|}
\hline White Walnut Bark & Boiled, crushed & $\begin{array}{l}\text { Applied to sore } \\
\text { joints }\end{array}$ \\
\hline Wild Black Cherry & Bark, Inner Bark, & Rheumatism; gout;
\end{tabular}

(Prunus serotina) Leaves, Twigs arthritis Bolyard, J. L. (1981). Medi

\begin{tabular}{l|l|l} 
Wild Yam & Tuber & Rheumatism
\end{tabular}

\begin{tabular}{|c|c|c|c|}
\hline Willow & Twigs, chewed on & Body pain & $\begin{array}{l}\text { Stephenson, S. L. (2013). } \\
\text { A }\end{array}$ \\
\hline \multirow[b]{2}{*}{$\begin{array}{l}\text { Willow (Salix sp) } \\
\text { Willow/Red Root }\end{array}$} & Bark & Rheumatism & $\begin{array}{l}\text { Amjad, H. (2006). Foll } \\
\text { Med }\end{array}$ \\
\hline & Bark & Rheumatism & $\begin{array}{l}\text { Wilson, C. R. and W. R } \\
\text { Ferr }\end{array}$ \\
\hline Wintergreen & $\begin{array}{l}\text { salves, of leaves } \\
\text { and oil }\end{array}$ & Rheumatism & $\begin{array}{l}\text { Crellin, J. K., et al. (1990). } \\
\text { H }\end{array}$ \\
\hline Witch Grass ("Quack") & Tea, of roots & Rheumatism & Shelton, F. (1965). Pionee \\
\hline
\end{tabular}




\section{Appendix C.}

\section{Literature Review - Prevalence of Herbal Use in General US population (2002-2012)}

3,985 Records identified Dec 2012 through AgeLine, PsycArticles, Psyclnfo, PubMed Medline, Scopus, AltHealthWatch using terms "CAM Survey," "CAM Use," "CAM Usage," "Botanical," "Herb,", "Biological Supplement," "Herbal Remedy," or "Herbal Remedies," and NOT "Intervention," or "Trial;" Option 'Health Professions' and Geographic Location 'United States' selected when available (Publication range 2001-2012; samples $>500$ ) --> Total studies reviewed $=34$ (including 11 retrieved

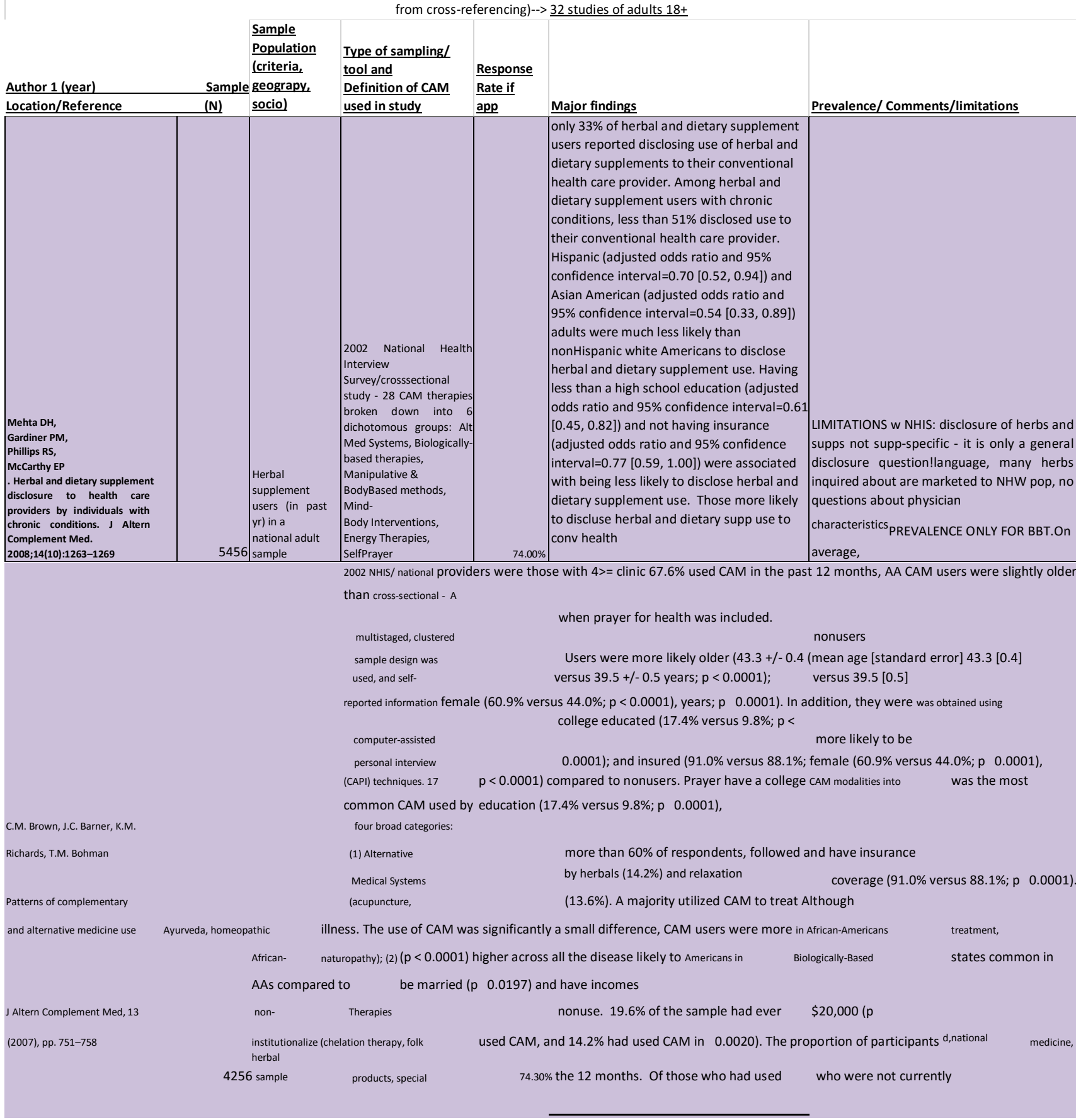




\begin{tabular}{|c|c|c|c|c|c|c|}
\hline $\begin{array}{l}\text { K.T. Xu, T.W. Farrell } \\
\text { Determinants of utilization: } \\
\text { the complementary and } \\
\text { substitution between } \\
\text { unconventional and mainstream } \\
\text { medicine among racial and ethnic } \\
\text { groups }\end{array}$ & 46,673 & 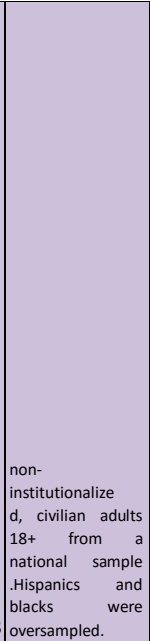 & $\begin{array}{l}\text { The Medical } \\
\text { Expenditure Panel Survey in } \\
1996 \text { and } 1998-12 \text { CAM } \\
\text { modalities. sampling frame } \\
\text { for the household survey } \\
\text { component was drawn from } \\
\text { respondents in the National } \\
\text { Health Interview Survey. } \\
\text { chiropractics massage } \\
\text { therapy, herbal remedies, } \\
\text { spiritual healings or } \\
\text { prayers, nutritional } \\
\text { advice, acupuncture, } \\
\text { meditation/imagery/ } \\
\text { relaxation, homeopathy, } \\
\text { traditional medicine, } \\
\text { biofeedback training, } \\
\text { hypnosis, and others. }\end{array}$ & & $\begin{array}{l}\text { CAM - herbals - in the last year, } 48.9 \% \\
\text { Multivariate analyses were conducted to } \\
\text { test whether each type of CAM and MSM } \\
\text { (mainstream med) were complements or } \\
\text { substitutes within a racial and ethnic } \\
\text { group, controlling for respondents' } \\
\text { sociodemographics and health. More } \\
\text { complementary relationships between } \\
\text { CAM and physician visits were found in } \\
\text { Nonhispanic Whites and Asians than in } \\
\text { other groups. All significant relationships } \\
\text { betweenCAMtypes } \\
\text { and physician visits amongHispanics and } \\
\text { other races (predominantlyNative } \\
\text { American } \\
\text { Indians) were substitution. }\end{array}$ & $\begin{array}{l}\text { Herbal CAM was used by } 34.35 \% \text { nonhispanic } \\
\text { whites, } 33.31 \% \text { of Hipanics, } 18.83 \% \text { of Blacks, } \\
34.68 \% \text { of Asians, and by } 18.67 \% \text { of } \\
\text { respondents who stated they were a race of } \\
\text { 'other' ( } 90 \% \text { of which were Native American } \\
\text { Indians). Herbal use was top choice of CAM } \\
\text { for conditions cancer ( } 31 \%) \text {, emphysema } \\
(36 \%) \text {, arthritis ( } 38 \%) \text {, asthma ( } 35 \%) \text {, } \\
\text { depression and anxiety disorders ( } 38 \%) \text {. } \\
\text { Herbal medicine was found to be a } \\
\text { complement to MSM among Asians (marginal } \\
\text { effects=.984) and the substitutions of herbal } \\
\text { remedies for MSM within the Other group } \\
\text { was the strongest of all sig relationships } \\
\text { found in the study (marginal effects = -3.23). } \\
\text { for each racial/etnic group, there are one or } \\
\text { two rpedominant CAM types and the } \\
\text { preveenct types differ by race. } \\
\text { LIMITATION: ghome or over-the-counter } \\
\text { CAM, such as herbs, was not specified so } \\
\text { underestimates are likley! additionally }\end{array}$ \\
\hline
\end{tabular}

\begin{tabular}{|c|c|c|c|c|c|}
\hline 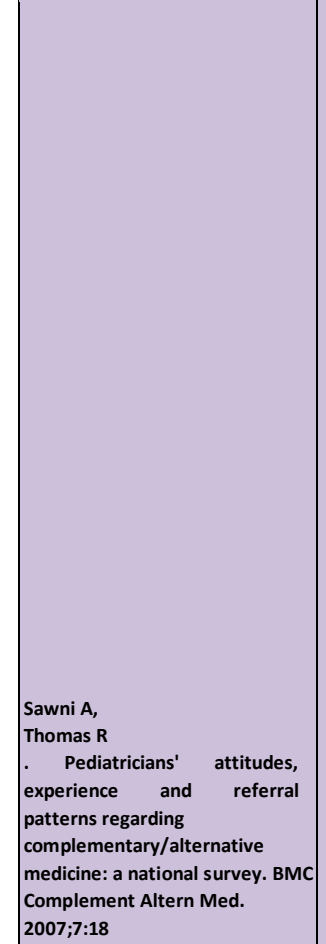 & $648 \mid \begin{array}{l}\text { fellows of the } \\
\text { American } \\
\text { Academy of } \\
\text { Pediatrics in } \\
\text { July } 2004 .\end{array}$ & 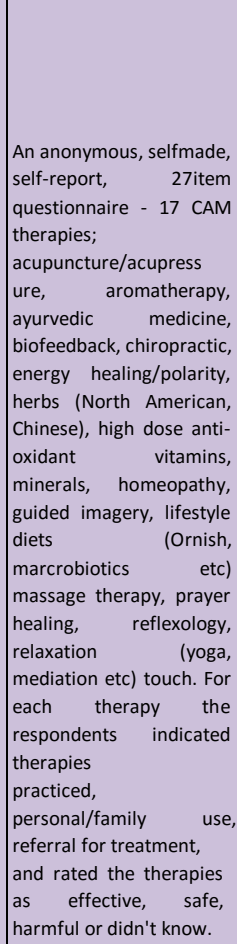 & & $\begin{array}{l}\text { The median age ranged from } 46-59 \text { yrs; } \\
52 \% \text { female, } 81 \% \text { Caucasian, } 71 \% \\
\text { generalists, \& } 85 \% \text { trained in the US. Over } \\
96 \% \text { of pediatricians' responding believed } \\
\text { their patients were using CAM. } \\
\text { Discussions of CAM use were initiated by } \\
\text { the family (70\%) \& only } 37 \% \text { of } \\
\text { pediatricians asked about CAM use as } \\
\text { part of routine medical history. Majority } \\
\text { (84\%) said more CME courses should be } \\
\text { offered on CAM and } 71 \% \text { said they would } \\
\text { consider referring patients to CAM } \\
\text { practitioners. Medical conditions referred } \\
\text { for CAM included; chronic problems } \\
\text { (headaches, pain } \\
\text { management, asthma, backaches) (86\%), } \\
\text { diseases with no known cure ( } 55.5 \% \text { ) or } \\
\text { failure of conventional therapies (56\%), } \\
\text { behavioral problems (49\%), \& psychiatric } \\
\text { disorders ( } 47 \% \text { ). American born, US } \\
\text { medical school graduates, general } \\
\text { pediatricians, \& pediatricians who } \\
\text { ask/talk about CAM were most likely to } \\
\text { believe their patients used CAM (P< } \\
0.01 \text { ). } 6 / 3 / 2013 \text { Pediatricians who talk to } \\
\text { patients about CAM are generalists rather } \\
\text { than a subspecialists and in }\end{array}$ & $\begin{array}{l}\text { lith } \\
\text { herbs and megavitamins were used } \\
\text { personally by } 17 \% \text { of the pediatricians } \\
\text { although herbs was the therapy believed } \\
\text { to be the most harmful (61\%). } 5 \% \text { practice } \\
\text { using herbs, } 17 \% \text { use on self or within their } \\
\text { families, } 10 \% \text { refer for herbs, } 52 \% \text { believe } \\
\text { herbs may be effective, and } 15 \% \text { believe } \\
\text { they are safe. LIMITATION: they didn't } \\
\text { include geographic location in their } \\
\text { analusis! also, this survey could have } \\
\text { assessed nurse practitioners and family } \\
\text { physicians but did not, and could have } \\
\text { gathered more info about CAM use for } \\
\text { specific therapies. }\end{array}$ \\
\hline
\end{tabular}




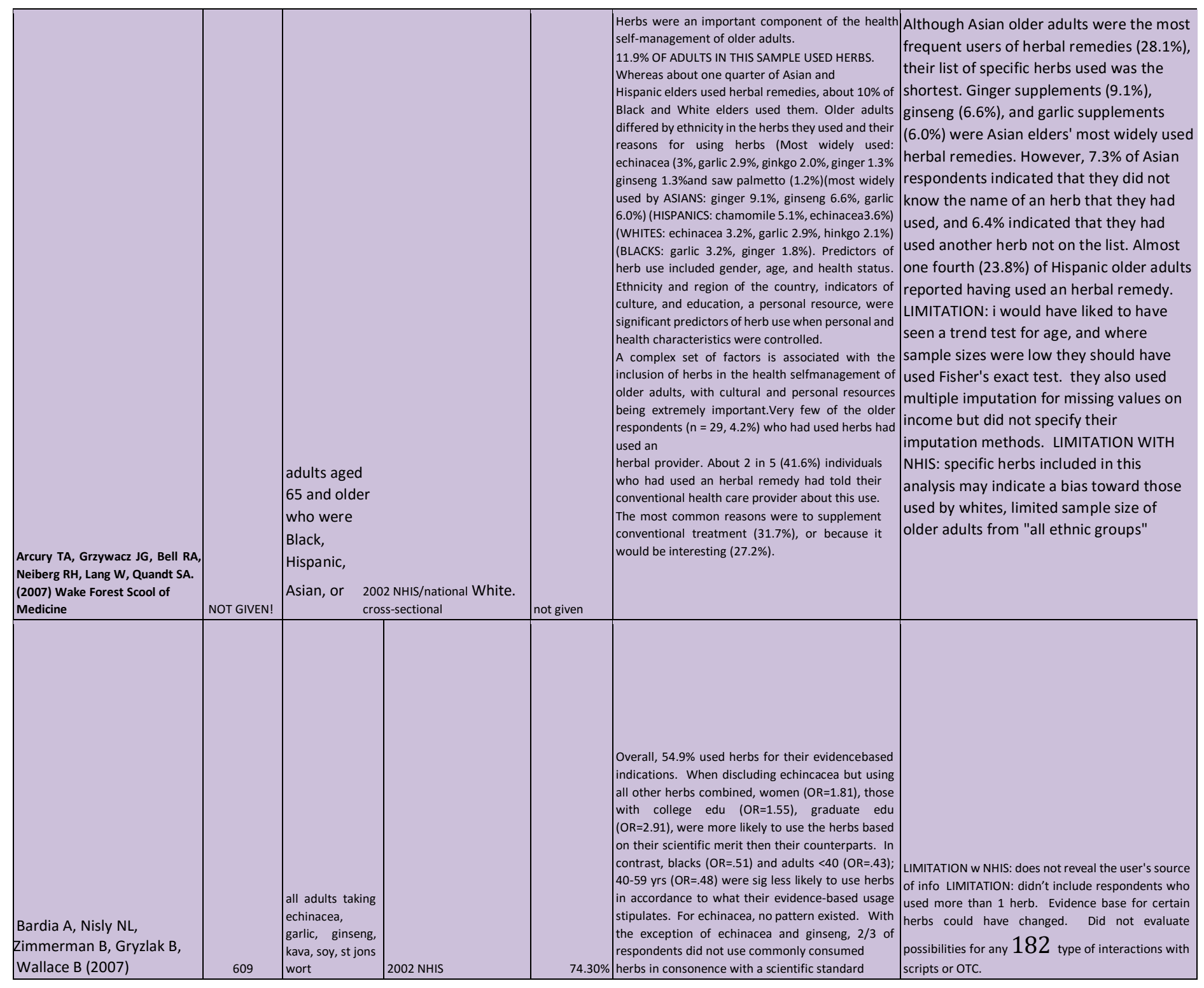

\begin{tabular}{|c|c|c|c|c|c|c|}
\hline $\begin{array}{l}\text { Barnes, PM, and Bloom, B } \\
\text { (2008) }\end{array}$ & $\begin{array}{c}75,764+ \\
31044\end{array}$ & $\begin{array}{l}\text { all children } \\
\text { and adults in } \\
\text { the US }\end{array}$ & $\begin{array}{l}2002 \text { NHIS (adults only) } \\
\text { and } 2007 \text { NHIS (adults and } \\
\text { children) }\end{array}$ & $\begin{array}{l}87.1 \% \text { in } \\
2007 \text { and } \\
74.3 \% \text { in } \\
2002\end{array}$ & $\begin{array}{l}\text { In } 2007 \text {, the CAM therapies most commonly used by } \\
\text { US adults in the past year were nonvitamin, } \\
\text { nomineral BBT (17.7\%). These were also the most } \\
\text { common CAM therapies used by children in the } \\
\text { rpevious year (3.9\%). Children whose parents used } \\
\text { CAM were about } \\
2 x \text { as likely as all US children to have used BBT ( } 9.2 \% \\
\text { and } 3.9 \%) \text {. In } 2007 \text {, one of the most commonly } \\
\text { used BBT by adults was echinacea }(19.8 \%) \text {, and by } \\
\text { children for health reasons was echinacea (37.2\%) } \\
\text { and a combination herb pill (17.9\%). For adults } \\
\text { younger than } 65 \text { yrs, in both } 2002 \text { and } 2007 \text {, those } \\
\text { with provate health insurance were more likely than } \\
\text { those with public health insurance or uninsured } \\
\text { adults to use biologically-based therapies. }\end{array}$ & $\begin{array}{l}\text { The NHIS CAM supplement changed from } 2002 \text { to } \\
\text { 2007. traditional healing traditions were added in, } \\
\text { while the \# of diseases treated with CAM was } \\
\text { expanded by } 8 \text {. The section on BBT was expanded by } \\
\text { 10 and an additional component of 'use in the last } 30 \\
\text { days' was added. Lastly, questions on CAM use on } \\
\text { children were added. }\end{array}$ \\
\hline
\end{tabular}




\begin{tabular}{|c|c|c|c|c|c|c|}
\hline $\begin{array}{l}\text { Bhargava, Vibha, hong Gong- } \\
\text { Soon, Montalto, C.P. (2012) } \\
\text { University of Georgia, lowa } \\
\text { State University, \& Ohio State } \\
\text { University }\end{array}$ & 23,393 & \begin{tabular}{|l} 
nationally \\
representative \\
sample adults
\end{tabular} & $\begin{array}{l}2007 \text { National } \\
\text { Health Interview } \\
\text { Survey/cross-sectional } \\
\text { study }\end{array}$ & $\begin{array}{l}\text { not } \\
\text { quantitative ly } \\
\text { stated in } \\
\text { article }\end{array}$ & $\begin{array}{l}\text { Total Respondents more likely to live in the } \\
\text { South }(36.7 \%) \text {, make } \$ 65 \mathrm{k} \text { more per year }(37.9 \%) \text {, } \\
82.95 \% \text { had health insurance, more likely to have } \\
\text { an education less than college }(73.3 \%) \text {. This study } \\
\text { highlights the need for more data with information } \\
\text { on factors relevant to explaining CAM use such as } \\
\text { price of self-care and practitioner-based CAM and } \\
\text { health insurance coverage for practitionerbased } \\
\text { CAM for application of a health economic model. }\end{array}$ & $\begin{array}{l}\text { nearly } 17 \% \text { of respondents had used herbs } \\
\text { within rpevious } 12 \text { months. those who } \\
\text { worked less than full time were more likely } \\
\text { to use herbs compared with those who } \\
\text { worked full time or more. individuals } 65 \\
\text { and older were more likely to use herbs } \\
\text { than those aged } 35-44 \text {. The predicted odds } \\
\text { of use of herbs among those who exercised } \\
\text { were almost } 77 \% \text { higher than those who } \\
\text { did not exercise. The predicted odds of use } \\
\text { of chiropractic and herbs were lower } \\
\text { among smokers than nonsmokers. The rate } \\
\text { of herbal use among the entire sample was } \\
17 \% \text {. Most prevalent herbal usage was in } \\
\text { the South (32.3\%), an income of } \$ 65 \mathrm{k}+ \\
\text { (45.5\%), had health insurance (86.1\%), } \\
\text { worked full tim or more (47.2\%), had } 1-2 \\
\text { chronic health conditions (45.2\%). } \\
\text { Although } 87.3 \% \text { of those who used herbs } \\
\text { self-described their health status as } \\
\text { 'Excellent', 'Very good' or 'Good', } 21.7 \% \\
\text { said they were depressed. }\end{array}$ \\
\hline $\begin{array}{l}\text { Davis MA, West AN, } \\
\text { Weeks WB, Sirovich BE } \\
\text { (2011) }\end{array}$ & 23393 & $\begin{array}{l}\text { national adult } \\
\text { sample 18+ }\end{array}$ & 2007 NHIS & & \multicolumn{2}{|c|}{$\begin{array}{l}11.7 \% \text { of adults used herbals and } \\
\text { nonvitamin supps for helath promotion } \\
\text { purposes, which was in the top two } \\
\text { most common modalities used for } \\
\text { promotion.stat sig diffs between LIMITATION w NHIS: missing data for treatment } \\
\text { and health promotion users, } \\
\text { obese, active ANALYSIS. health status measures are selfsmoker, any func limitation. } \\
\text { reported and so susceptible to medical or therefore, there are two distinct types } \\
\text { psychological conds. They also stated of CAM users. Health promotion users that } \\
\text { health promotion users have rates of have sig better self-reported health conv } \\
\text { health services use comparable to status and have healthier behaviors those of non- } \\
\text { users but was a poisson reg overall when compared } w \text { tx users. poss given the } \\
\text { dataset? Not sure. }\end{array}$} \\
\hline
\end{tabular}




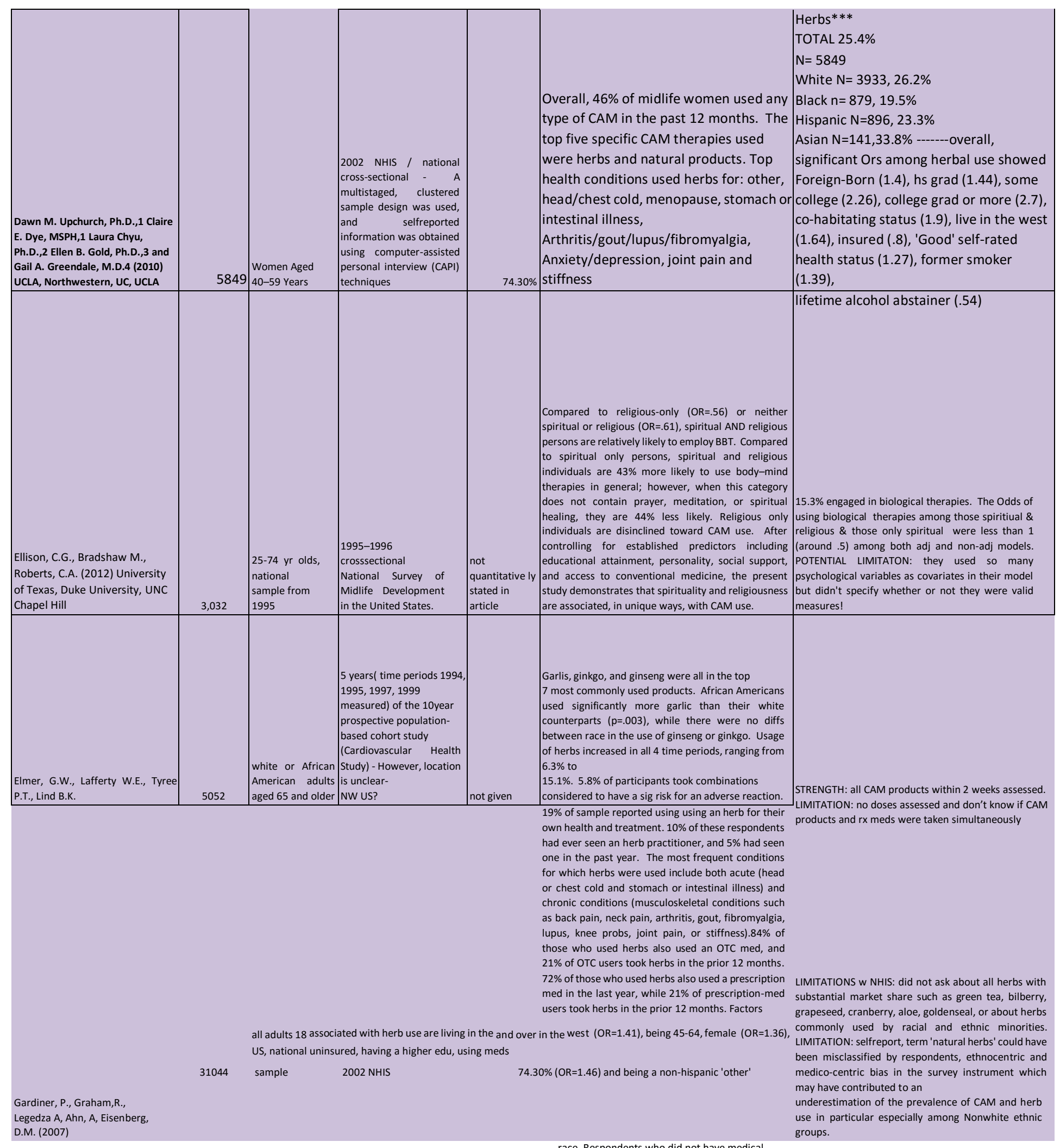




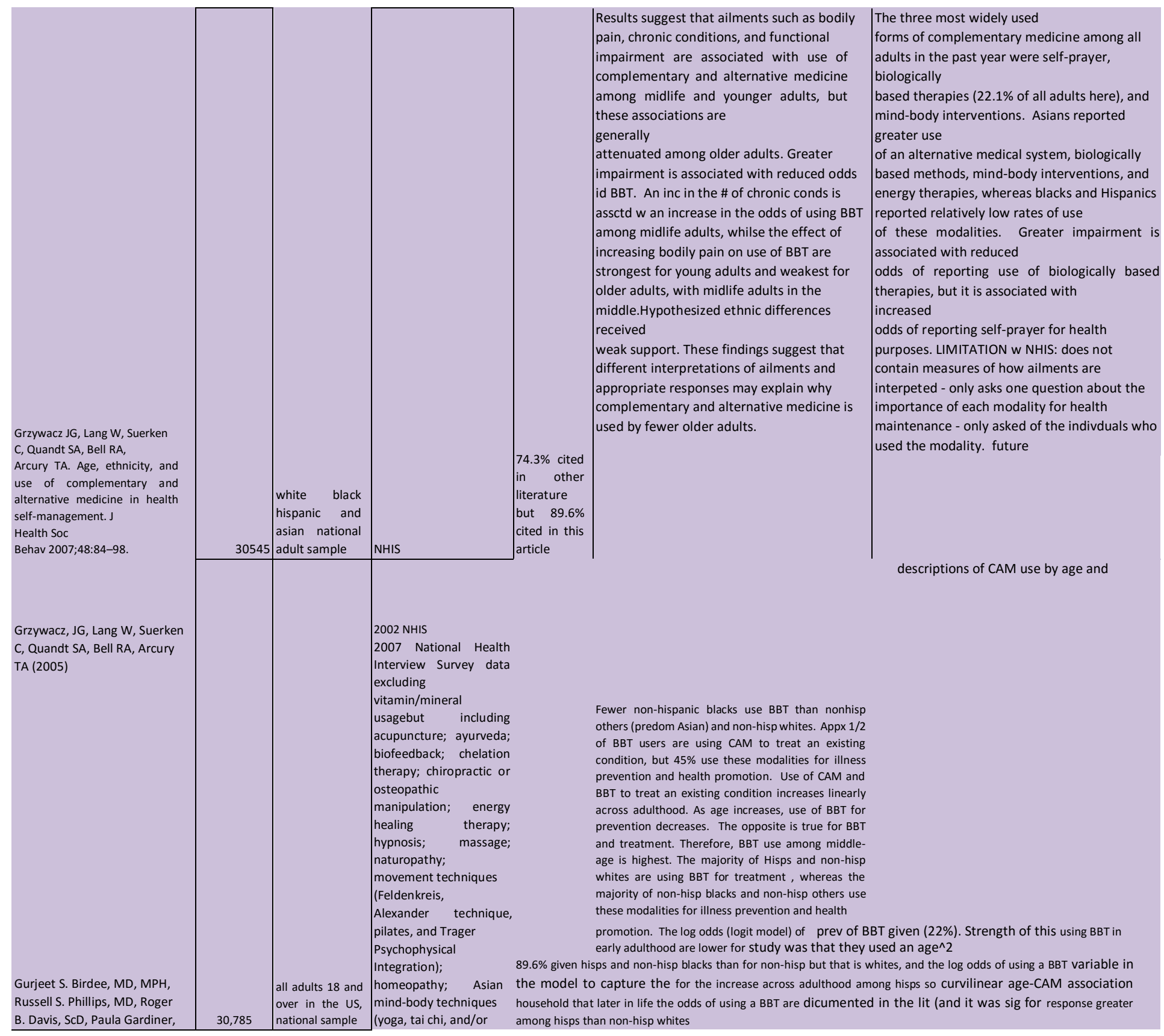


MD, MPHc (2010) Harvard,

Beth Israel, Boston University
BBT!) and they addressed missing data! ratehere (and blacks). after midlife, the log odds of they used using BBT decrease for both non-hisp whites Limitation potential retrospective recall other and blacks by age, gender, and edu.
In 2007, pediatric CAM users were more likely to take prescription medications, have a parent who used CAM, and have chronic

conditions such as anxiety or stress, biologically-based therapies were used by $4.7 \%$ musculoskeletal conditions, dermatologic of the sample. Use of biologically based conditions, or sinusitis (significant therapies correlated with being an adolescent confounding factors were controlled for). CAM versus being an infant or toddler, white versus use was least common in the South and most non-Hispanic black or Hispanic, living in the common in the West. Research is required to West versus the South, and parents being guide pediatricians in making college educated versus parents completing recommendations on CAM modalities for less than high school. Biologically based children including potential risks and/or therapies were also associated with delays in benefits and interactions with conventional health care because of difficulties with access, therapies. There was nearly equal CAM use and medical conditions including insomnia, between boys and girls, higher use among non-fever, reflux, and sinusitis. LIMITATIONS: data Hispanic white patients, higher use among collected from an adult proxy which is educated families, and regional variations susceptible to recall bias. \# of children with chronic conds small, limiting power,

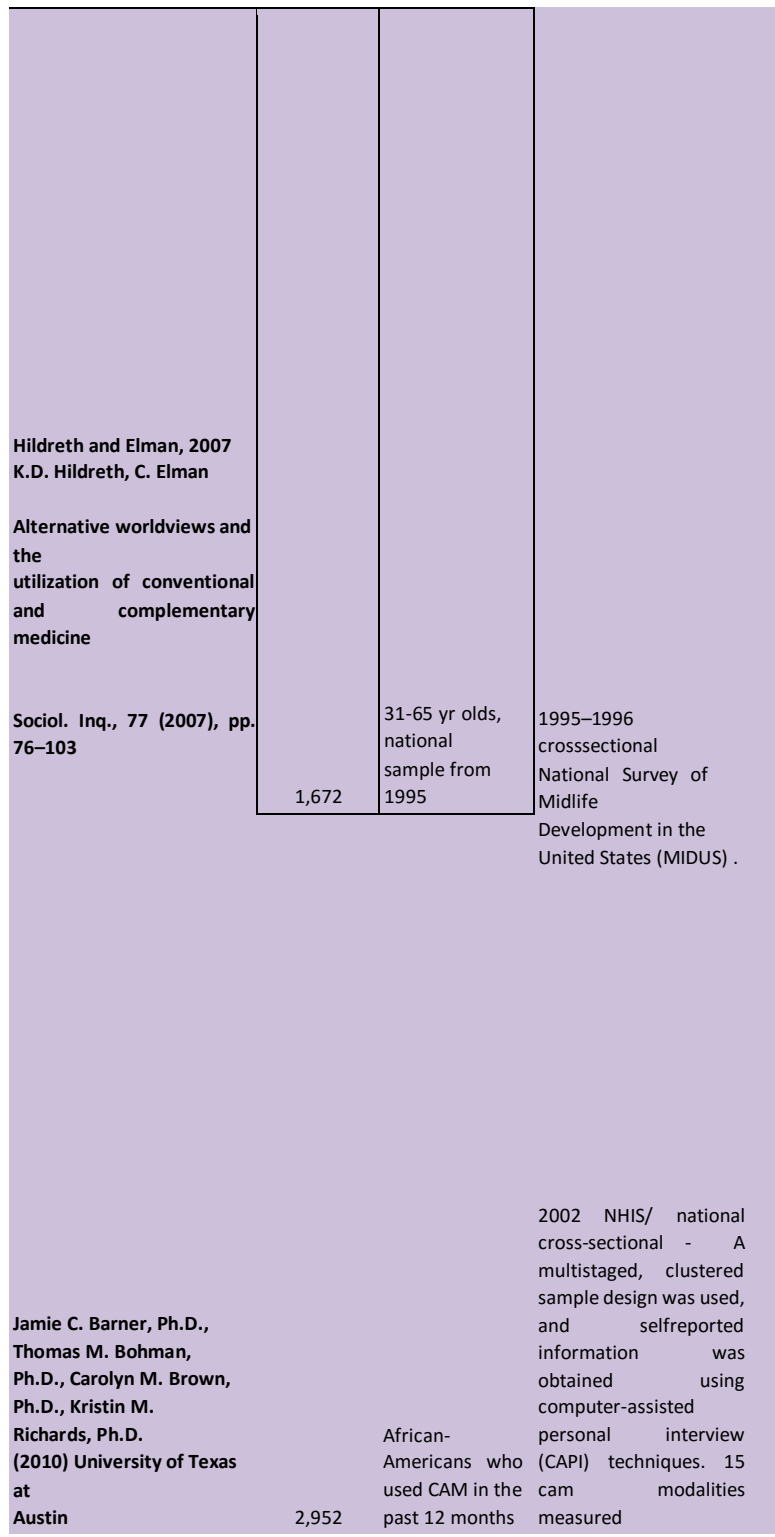

Higher self-rated spirituality is associated with a greater probability of CAM use and the use of more techniques. Although higher self-rated religiosity does not distinguish CAM users from nonusers, it is associated with adopting significantly fewer techniques. Assuming that the religious versus spiritual worldviews are differentiated by religious individuals' greater embeddedness in community and institutional "orthodoxies" (e.g., adherence to a set of prescribed beliefs), a religious worldview appears to carry over to constrain

the use of nonconventional practices and providers. It may also be that CAM's tendency to secularize otherwis sacred techniques makes this type of care less attractive to these users (Goldstein 1999). Openness shows itself to be a

$60.80 \%$

Limitation: interpretation of tables confusing: means were given for categorical variables, the diffs between model 1 and 2 (and 3!) were never explained, and OR's werent given Herbal WEIGHTED:

past 12 month $\mathrm{N}=3,394,228$ cam for treatment: $n=1,660,353$ (48.9\%) cam for

Approximately 1 in $5(20.2 \%)$ who used CAM in the prevention: past 12 months used CAM to treat a $n=1,709,464(50.4 \%)$ specific condition. Less than $50 \%$ (48.9\%) of AA Folk medicine exception of folk medicine. Ten of the 15 CAM WEIGHTED: exception of folk medicine. Ten of the 15 CAM
modalities were used primarily for treatment by past 12 month African-Americans. CAM for treatment was $\mathrm{N}=27,141$ cam for significantly $(P<.05)$ associated with the following treatment: $n=21,259$ factors: graduate education, smaller family size, treatment: $n=21,259$
higher income, region (northeast, midwest, west $(78.3 \%)$ cam for higher income, region (northeast, midwest, west $(78.3 \%)$ physician visits, less likely to engage in preventive $n=5,882(21.7 \%) \quad$ LIMITATION: didnt care, more frequent exercise behavior, more break down CAM modality by table activities of daily living (ADL) limitations, and neck break down CAM LIMATION w NHIS: CAM for treatment used CAM in the past year were treating a specific answer 'no' does not necessarily imply condition. Alternative medical systems, prevention, does it? wording of these manipulative and body-based therapies, and folk prevention, does it? wording of these medicine, prayer, biofeedback, and energy/Reiki questions "home remedies" and culturally
were used most often. 


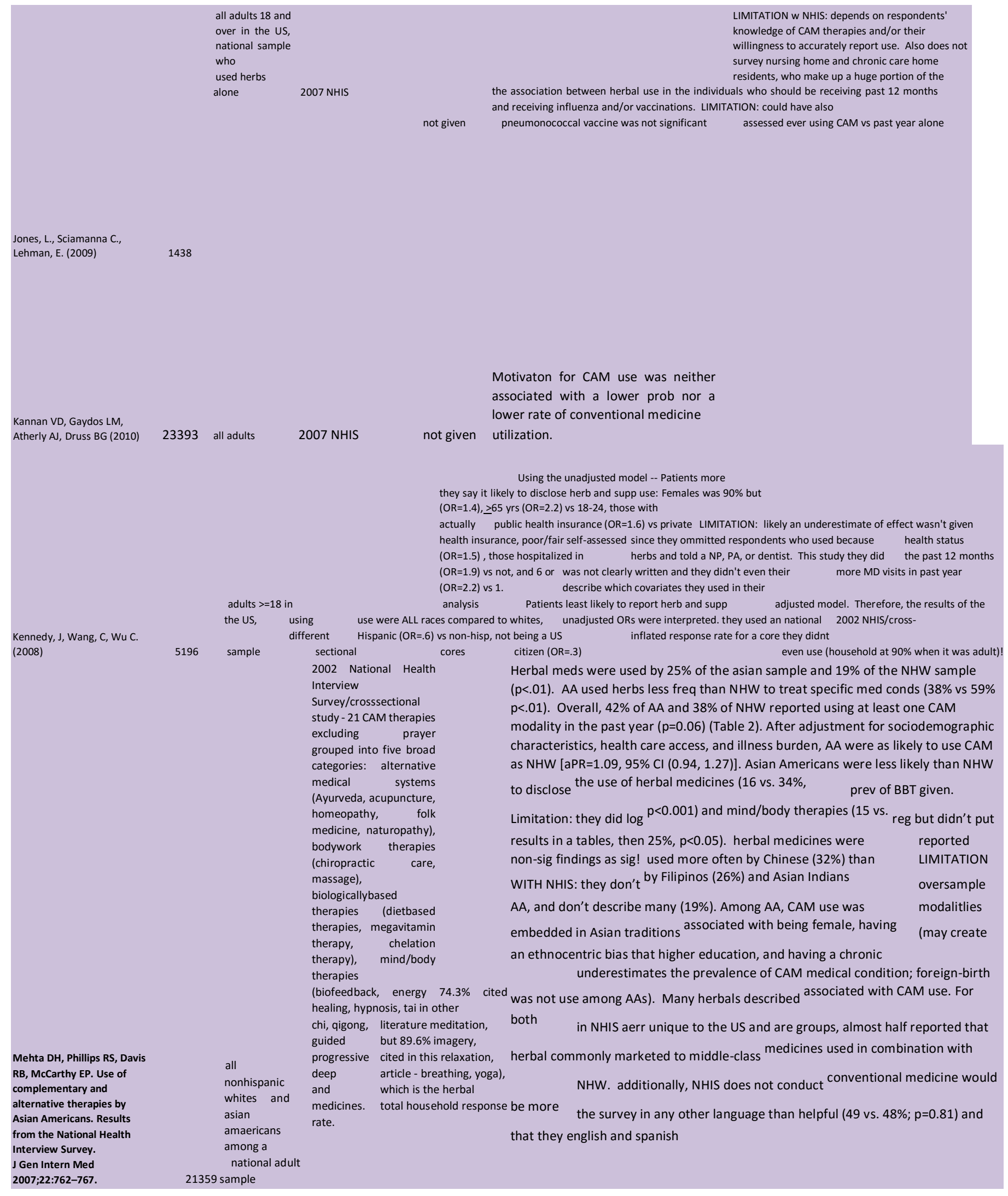


self-made survey used herbal medicines because they based on the

revised

Health Promotion

Lifestyle, Profile II.

CAM use was

derived from

five questions

(three close-ended

and two open-

American ended) that

Older age, female gender, unmarried, Indian

adults assessed and higher income was associated with in seven U.S.

respondents' use of dietary and herbal cities with vegetarian status:

supplements. $30.4 \%$ used dietary high "Are you a and herbal

supplements. use of

concentration vegetarian?" "Are herbal and dietary of Als: you currently taking

supplements among males indicated

Houston, TX; dietary and herbal they were older, moreeducated, had higher English

Phoenix, AZ; supplements?" and proficiency, and were tobacco Washington, "Do you use

any users compared to AI females.

DC; Boston, alternative medical However, females were 1.8 times

MA; San systems (AMS, also more likely to Alt med systems and

Misra, Ranjita :

Diego, $C A$; referred to as

herbal supplements than males (table

Balagopal,

Padmini ; Klatt,

Edison, NJ;

Whole Medical

Maryanna ;

and Systems) such as

Geraghty, Maureen

(2010)

Texas A\&M

Parsippany, Ayurveda,

NJ.

homeopathic

4). married respondents

were 2.8 times more likely to use

strength: they did cronbachs a on

dietary and herbal supplements than

subscales!, assessed for inter-rater $37 \%$ non-married. reliability,

Nahin RL, Barnes PM,

Stussman BJ, Bloom B.

Costs of complementary and alternative medicine (CAM) and frequency of visits to CAM

practitioners: United

States, 2007. Natl Health

Stat Rep. 2009;

(18): 1-14.

treatment, and

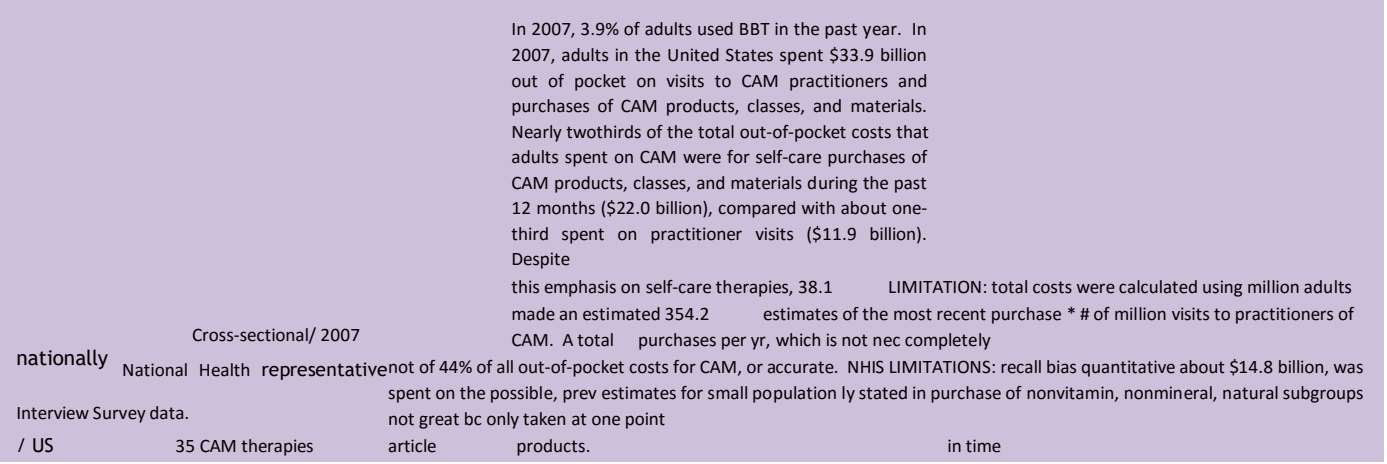


Nahin, R, Dalhammer, JM, Stussman BJ (2010)

Nahin, RL, Dahlhamer JD, Taylor BL, Barnes PM, Stussman BJ, Simile CM, Blackman MR, Chesney MA, Jackson M, Miller $\mathrm{H}$, and McFann KK (2007) all adults 18 and over in the US, national 31044 sample 2002 NHIS OR's only given for general CAM use and not $74.30 \%$ BBT

Engaging in liesure time physical activity, having consumed alcohol in one's life but not being a current heavy drinker, and being a former smoker are independently associated with the use of CAM. Obese are slightly less likely to use CAM than individuals with a healthy BMI. No sig $74 \%$ bw flu vaccine and CAM use. national adult 31044 sample 18+ 2002 NHIS
Neiberg, Rebecca H. ; Aickin, Mikel ; Grzywacz, Joseph G. Lang, Wei ; Quandt, Sara A. ; Bell, Ronny A. ; Arcury, Thomas A. (2011) Division of Public Health Sciences, Department of Biostatistical Sciences, , Winston-Salem, NC.

College of Medicine, , Tucson, AZ.

Department of Family and Community Medicine, Winston-Salem, NC.

Division of Public Health

Sciences, Department of Epidemiology and Prevention, , Winston-Salem, NC. national adult 27,862 sample $18+\quad 2002$ NHIS
19.3\% of adults did not use conventional care within the past year despite the fact that $38.4 \%$ of these individuals had one or mroe health needs, with almost $25 \%$ having a serious chronic or acute medical condition. Instead of conventional care, $24.8 \%$ of these individuals used CAM. $68.5 \%$ of those only using CAM used BBT. $24.8 \%$ of those not using conventional care used some form of CAM, with $12 \%$ reporting one or more health needs and using CAM. Of all individuals using CAM only, $21.6 \%$ thought conventional med treatments wouldn't help, and $20.4 \%$ said conv med treatments were too expensive. The majority $(54.1 \%)$ said they thought it would be interesting to try. strengths: assessed colinearity to compare models

Biologically-based therapies were observed in $5.5 \%$ of those who used a single modality with a significan probablity that biologically based and other CAM types are linked. Among the $20.6 \%$ of linkset users, singificant associations were seen between biologically-based users and $10.6 \%$ of those who used mind-body therapies, among other combinations of CAM use (less than $10.6 \%$ ). There was a significant difference by genderbetween those who used both biologicallybased therapies and mindbody therapies (male $5.6 \%$, fem $7.6 \%$ ), those who used biologically-based therapies + alt health systems + mindbody therapies (males $.4 \%$ fem $.8 \%$ ), and those who used all 4 CAM modalities (biologically-based, alt health systems, manipulative methods, and mindbody therapies) (males .5\%, fem $.8 \%)$. Among those who used both alt health systems and biologically-based therapies, significant diffs Linkset analysis, a method of estimating cooccurrence occurred for whites $>$ blacks \& whites $>$ hispanics. For

beyond chance to identify possible sets of CAM use. Results: Most adults use CAM therapies from a single category. Approximately $20 \%$ of adults combined two CAM categories, with the combination of mind-body therapies and biologically based therapies estimated to be most common. Only $5 \%$ of adults use therapies representing three or more CAM categories. Combining therapies across multiple CAM categories Combining therapies across multiple CAM categories was more common among those 46-64, women, whites, and those with a college education. 作 researchers to refine descriptions of CAM use in the be $45-54$ yrs old.

adult population. Most adults do not use a wide assortment of CAM; most use therapies within a single CAM category. Sets of CAM use were found to differ by age, gender, ethnicity, and education in ways consistent with previous

\begin{tabular}{|c|c|c|c|}
\hline & & 2002 NHIS + 2003 MEPS & $\begin{array}{l}\text { interaction effects not possible due to low } \\
\text { sample sizes of other forms of CAM measured. RCTS mremain nec to evaluate the saftey and efficacy } \\
\text { of CAM in older adults STRENGTHS: dealth w missing income issue by using a dichotomous measure } \\
\text { which may have helped BBT was used by } 20.4 \% \text { of this sample. The use a little \& reliability (a) of the } \\
\text { functional score of Biologically based therapies predicted assessed, LIMITATION: but not for the } \\
\text { physical better functional status (using a validated HRQoL and mental HRQoL. analysis reports scale } \\
\text { and adjusting for many factors) ( }-1.14 \text { short-term } 1 \text { year effects, interaction effects lowered func } \\
\text { status score between those were not included due to low rates of some using BBT vs no BBT) ( } p<.01 \text { ) } \\
\text { in } 1 \text { year modalities }\end{array}$ \\
\hline Nguyen, H.T., Grzywacz, JG & & $\begin{array}{l}\text { longitudinal } 89.6 \% \text { for component on } \\
\text { the NHIS; not }\end{array}$ & \\
\hline Lang, W, Walkup M, Arcury, T & & adults aged $55+$ same sample from given for & \\
\hline 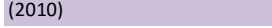 & 1683 & nationally 2002 NHIS & \\
\hline
\end{tabular}


Nguyen, Long T., Davis, Roger B., Kaptchuk, Ted J., \& Phillips, Russell S. (2011) National Center for Health Statistics

cciano, F., Dwyer, J.T.,

Radimer, K.L., Wilson,

D.H., Fisher, K.D., Thomas,

P.R., Yetley, E.A.,

Moshfegh A.J., Levy P.S.,

Nielsen, S.J., Marriott,

B.M. (2007)
2007 National

Health Interview

Survey/cross- not

nationally

representative

23,393 sample

qua and health status article

General CAM usage ly stated in

$37 \%$ of U.S. adults used complementary and alternative medicine and $63 \%$ did not use any CAM. Compared to those who did not use CAM, CAM users were more likely to rate their health as 'Excellent' (adjusted-odds ratio $(A O R)=1.14$, $95 \% \mathrm{Cl}=[1.03,1.26])$. Conclusion

We found a significant association between CAM use and self-rated excellent health and health improvement over the prior year. Prospective trials are required to determine whether CAM use is causally related to excellent health status and better health than in the prior year.

\section{NOT ASSESSED, ABSTRACT ONLY AVAIL}

These data the first to include info on the use of botanicals in addition to nutrient-containing detary supps among children. I wish they had $.8 \%$ prevalence for any botanical supplements, made a table of the botanicals, however - they with no sig diffs by sex. The most commonly used were only available via reporting through the botanical supplements were ginseng, echinacea, write-up. STRENGTH of NHANES is info from ginkgo, and grape seed extract (they also inc citrus label recorded directly by trained pros in bioflavenoids which are not a natrual herb or interviewers homes, LIMITATIONS of NHANES extract). Few children

99-2000 and 20012002 NHANES national All kids $<=18$ yrs survey (some pops 10136 age not given

younger than 3 had used botanical supplements, and the maximum prevalence was only $1 \%$ in teenagers.

$19.3(n=488)$ of participants used at least herb/natural product and there were stat sig diffs by gender (women), race (whites), and edu (better eductated) in bivariate analyses. Among those who used both conventional medication and herbs/natural products, more than 1 in 5 (97 [21.9\%]) were using a combination of products associated with a

Population-based epidemiological study Johnston County Osteoarthritis Project (1991-2004) survey TWO COHORTS COLLAPSED TOGETHER - which contained a list of 51 products including herbs and botanical products,

Philip J. Gregory,

PharmD; Rajul A. Patel, PharmD, PhD; Linda L. Norton, PharmD;

Leigh F. Callahan, PhD;

Joanne M. Jordan, MD, MPH (2009) UNC Chapel Hill, Creighton University, Thomas J Long School of Pharm and Health Sciences, University of the Pacific, Thurston Arthritis Research Center natural products

civilian, obtained from animal noninstitutiona sources natural lized, white or hormones (eg,

African phytoestrogen

American pills), and foods that residents, aged are sometimes 45 years$$
\text { or }
$$

consumed for older, of

Johnston medicinal purposes as

County, North query. vitamins and

Carolina (AA's minerals not assessed

oversampled) potential interaction. Odds of exposure to a potential interaction'

were lower among people who had health insurance and increased

with the number of products used. A total of 168 potential interactions were identified. As shown in Table 4, 115 ofthe 168 potential interactions (68.5\%)

involved garlic and cytochrome P450 3A4 substrates (eg, amlodipine, atorvastatin, simvastatin, verapamil). Although the

severity of most ofthe 168 potential interactions was classified

as "moderate," 27 (16.1\%) were classified as

"high" in severity. The bivariate analyses indicate that the

odds of exposure to a potential interaction

increase as fatigue,

number of comorbidities, number of herbs used, number of

excluded all missing data so sample size for full OTC medications used, and number of prescription model (herb users who used at least one med as medications well) went down to 419. STRENGTH: extensive used increase. In addition, individuals with health array of natural herbs/products LIMITATION: insurance interaction data utilized as basis were caseare cross-sx data, use of proxies under 16 may , diffs in methodology and defs over time may limit the comparison of prevalence data
Upchurch DM , Chyu L, Greendale GA, Utts J, Bair YA, Zhang G, Gold E (2007)

$\begin{array}{ll}\text { national } & \text { adult } \\ \text { women sample } & \\ 18+ & 2002 \text { NHIS }\end{array}$

STRENGTHS: addressed income nonresponse and compared missing vs no missing: no diff in effects LIMITATION: missing values for smoking alcohol and BMI imputed. No Cis reported

BBT the 2nd most freq reported (23.8\%) after prayer. Foreign-born women were more likely to use BBT than US-born women (but remember they didnt control for confounders!) menopause in the top 10 conditions $74 \%$ only for BBT
LIMITATION w NHIS:underestimates likely in ethnic communities of women since traditional healers not yet inc in dataset. many trad practices maybe not considered "alternative" since they may be perceived as common in some communities, resulting in potential misclassification 


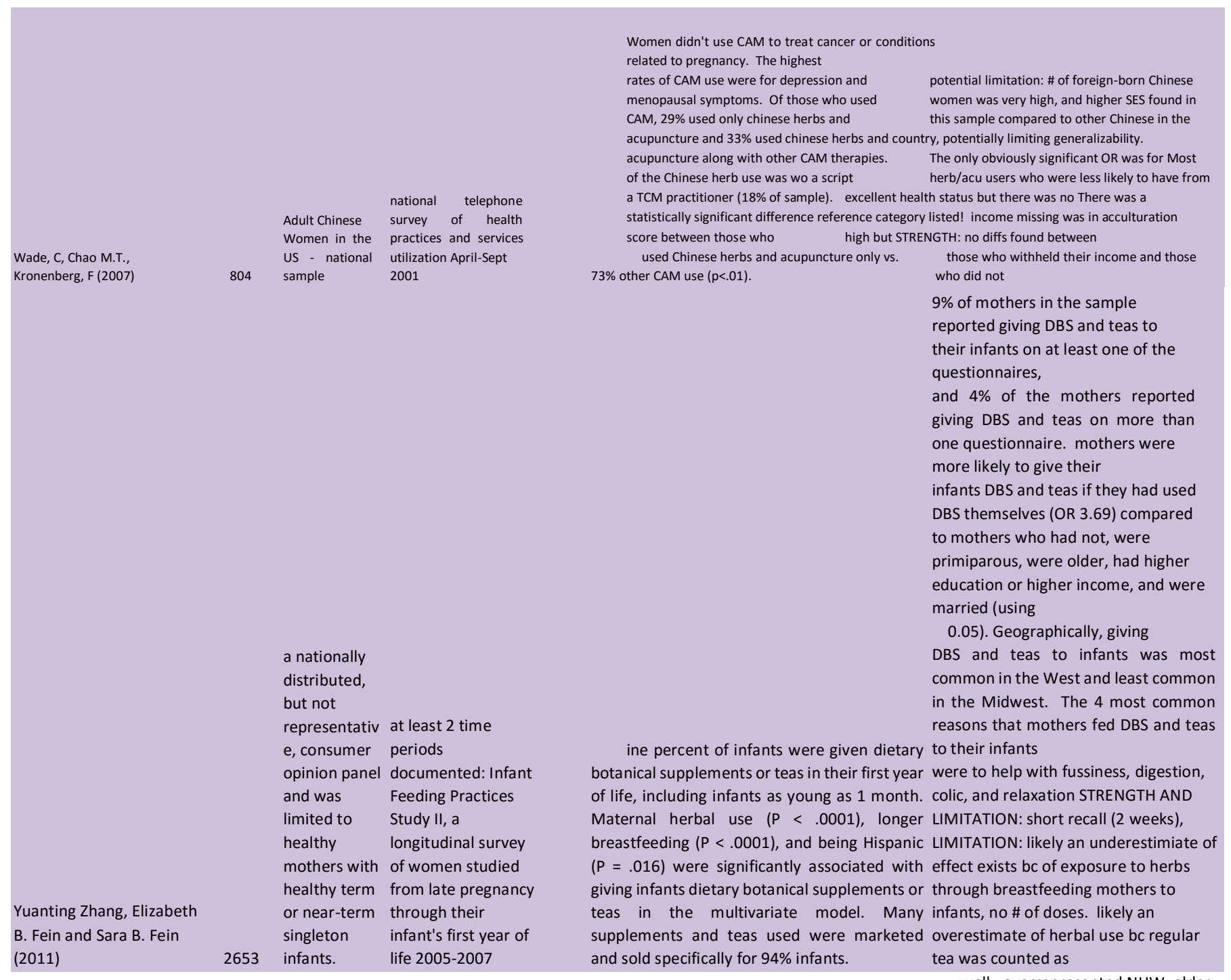

well. overrepresented NHW, older

\section{Appendix D.}




\section{WestlinginiaUniversity. \\ SCHOOC OF PUEUC HEALTH}

\section{Dear Patient,}

The WVU School of Public Health is conducting a study to learn more about the experience of people who suffer from pain. This research project is being conducted in partial fulfillment of the requirements for a doctoral dissertation.

The purpose of this survey is to understand which, if any, types of complementary health approaches (such as acupuncture, massage, yoga, supplements, etc.) you may use for pain. Please complete this survey only if you are a patient in this clinic and are at least 18 years of age.

Attached is a voluntary, anonymous survey which should take $10-12$ minutes to complete. Your status as a patient in this clinic will not be affected in any way by refusal to participate in this study. When you are finished, please drop the survey in the box located in the patient waiting room. You do not have to answer every question; if you only have the time to complete some of the survey, please do not hesitate to complete as much as you can.

This study has been reviewed and acknowledged by the West Virginia University Institutional Review Board.

We appreciate your taking the time to complete this survey so that we can better understand the ways in which people manage their pain.

Thank you for your help!

Sincerely,

Termeh Feinberg, MPH

Epidemiology Doctoral Student, WVU School of Public Health tmkuklinski@mix.wvu.edu

$304.293 .0659 / 267.444 .3775$ 
Chronic pain is often defined as any pain lasting more than 12 weeks. Chronic pain may arise from an initial injury, such as a back sprain, or there may be an ongoing cause, such as illness. Have you ever experienced pain lasting 12 weeks or longer?

口Yes

№

Are you currently experiencing chronic pain as defined in the previous question (pain lasting 12 weeks or longer)?

口Yes

No

I have experienced chronic pain before but am not experiencing chronic pain presently

FOR EACH QUESTION, PLEASE INDICATE YOUR LEVEL OF PAIN BY CIRCLING A NUMBER FROM 0 TO 10:

YOUR PAIN:

My current pain is

No pain: $\begin{array}{lllllllllll}0 & 1 & 2 & 3 & 4 & 5 & 6 & 7 & 8 & 9 & 10 \text { :Extreme pain }\end{array}$

During the past week, the best my pain has been is

No pain: $\begin{array}{lllllllllll}0 & 1 & 2 & 3 & 4 & 5 & 6 & 7 & 8 & 9 & 10 \text { :Extreme pain }\end{array}$

During the past week, the worst my pain has been is

No pain: $\begin{array}{lllllllllll}0 & 1 & 2 & 3 & 4 & 5 & 6 & 7 & 8 & 9 & 10 \text { :Extreme pain }\end{array}$

During the past week, my average pain has been

No pain: $\begin{array}{lllllllllll}0 & 1 & 2 & 3 & 4 & 5 & 6 & 7 & 8 & 9 & 10 \text { :Extreme pain }\end{array}$

During the past 3 months, my average pain has been

No pain: $\begin{array}{llllllllllll}0 & 1 & 2 & 3 & 4 & 5 & 6 & 7 & 8 & 9 & 10 \text { :Extreme pain }\end{array}$

YOUR FEELINGS: During the past week I have felt:

Afraid

Strongly Disagree: $\begin{array}{lllllllllll}0 & 1 & 2 & 3 & 4 & 5 & 6 & 7 & 8 & 9 & 10\end{array}$ :Strongly Agree

Depressed

Strongly Disagree: $\begin{array}{lllllllllll}0 & 1 & 2 & 3 & 4 & 5 & 6 & 7 & 8 & 9 & 10 \\ \text { :Strongly Agree }\end{array}$

Tired

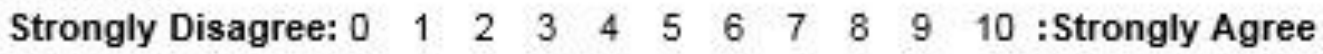
Anxious

Strongly Disagree: $\begin{array}{lllllllllll}0 & 1 & 2 & 3 & 4 & 5 & 6 & 7 & 8 & 9 & 10\end{array}$ :Strongly Agree

Stressed

Strongly Disagree: $\begin{array}{lllllllllll}0 & 1 & 2 & 3 & 4 & 5 & 6 & 7 & 8 & 9 & 10\end{array}$ :Strongly Agree 
YOUR CLINICAL OUTCOMES DURING THE PAST WEEK:

I had trouble sleeping

Strongly Disagree: $\begin{array}{lllllllllll}0 & 1 & 2 & 3 & 4 & 5 & 6 & 7 & 8 & 9 & 10\end{array}$ :Strongly Agree

I had trouble feeling comfortable

Strongly Disagree: $\begin{array}{lllllllllll}0 & 1 & 2 & 3 & 4 & 5 & 6 & 7 & 8 & 9 & 10\end{array}$ :Strongly Agree

I was less independent

Strongly Disagree: $\begin{array}{lllllllllll}0 & 1 & 2 & 3 & 4 & 5 & 6 & 7 & 8 & 9 & 10\end{array}$ :Strongly Agree I was unable to work (or perform normal tasks)

Strongly Disagree: $\begin{array}{lllllllllll}0 & 1 & 2 & 3 & 4 & 5 & 6 & 7 & 8 & 9 & 10\end{array}$ :Strongly Agree I needed to take more medication

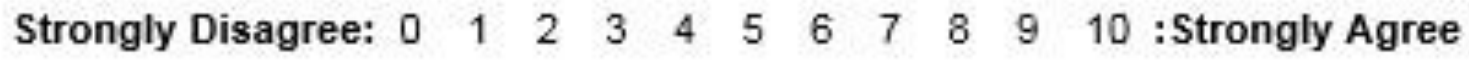

YOUR ACTIVITIES: During the past week I was NOT able to:

Go to the store

Strongly Disagree: $\begin{array}{lllllllllll}0 & 1 & 2 & 3 & 4 & 5 & 6 & 7 & 8 & 9 & 10\end{array}$ :Strongly Agree

Do chores in my home

Strongly Disagree: $\begin{array}{lllllllllll}0 & 1 & 2 & 3 & 4 & 5 & 6 & 7 & 8 & 9 & 10\end{array}$ :Strongly Agree

Enjoy my friends and family

Strongly Disagree: $\begin{array}{lllllllllll}0 & 1 & 2 & 3 & 4 & 5 & 6 & 7 & 8 & 9 & 10 \text { :Strongly Agree }\end{array}$ Exercise (including walking)

Strongly Disagree: $\begin{array}{lllllllllll}0 & 1 & 2 & 3 & 4 & 5 & 6 & 7 & 8 & 9 & 10\end{array}$ :Strongly Agree Participate in my favorite hobbies

Strongly Disagree: $\begin{array}{lllllllllll}0 & 1 & 2 & 3 & 4 & 5 & 6 & 7 & 8 & 9 & 10 \text { :Strongly Agree }\end{array}$ 


\section{PLEASE CHECK THE BOX IN EACH ROW TO DESCRIBE YOUR USAGE OF COMPLEMENTARY HEALTH APPROACHES FOR PAIN:}

\begin{tabular}{|c|c|c|c|c|c|c|c|}
\hline $\begin{array}{l}\text { Treatment/ } \\
\text { Prevention used } \\
\text { for pain? }\end{array}$ & $\begin{array}{l}\text { d do not } \\
\text { know } \\
\text { what } \\
\text { this is }\end{array}$ & $\begin{array}{l}\text { No, and I } \\
\text { do not } \\
\text { intend to } \\
\text { within } \\
\text { the next } \\
6 \\
\text { months }\end{array}$ & $\begin{array}{l}\text { No, but I } \\
\text { intend to } \\
\text { within the } \\
\text { next } 6 \\
\text { months }\end{array}$ & $\begin{array}{l}\text { No, but I } \\
\text { intend to } \\
\text { within } \\
\text { the next } \\
30 \text { days }\end{array}$ & $\begin{array}{l}\text { Yes, and } \\
\text { I have } \\
\text { for less } \\
\text { than } 6 \\
\text { months }\end{array}$ & $\begin{array}{l}\text { Yes, and } \\
\text { I have } \\
\text { for more } \\
\text { than } 6 \\
\text { months }\end{array}$ & $\begin{array}{l}\text { If Yes, } \\
\text { how } \\
\text { effective } \\
\text { is it in } \\
\text { managing } \\
\text { your } \\
\text { pain? }\end{array}$ \\
\hline \multicolumn{8}{|l|}{$\begin{array}{l}\text { Herbs/Botanicals } \\
\text { (such as } \\
\text { Echinacea, Black } \\
\text { Cohosh, etc.) }\end{array}$} \\
\hline \multicolumn{8}{|l|}{$\begin{array}{l}\text { Vitamins and/or } \\
\text { Minerals (such as } \\
\text { Vitamin C, } \\
\text { Magnesium, etc.) }\end{array}$} \\
\hline \multicolumn{8}{|l|}{ Probiotics } \\
\hline \multicolumn{8}{|l|}{$\begin{array}{l}\text { Other natural } \\
\text { products (such as } \\
\text { fish oil, lecithin, } \\
\text { etc.) }\end{array}$} \\
\hline \multicolumn{8}{|l|}{ Acupuncture } \\
\hline \multicolumn{8}{|l|}{ Massage therapy } \\
\hline \multicolumn{8}{|l|}{$\begin{array}{l}\text { Spinal } \\
\text { manipulation/ } \\
\text { Chiropractic }\end{array}$} \\
\hline \multirow{2}{*}{\multicolumn{8}{|c|}{$\begin{array}{l}\text { Tai chi/Qi gong } \\
\text { Yoga }\end{array}$}} \\
\hline & & & & & & & \\
\hline \multicolumn{8}{|l|}{ Meditation } \\
\hline \multicolumn{8}{|l|}{$\begin{array}{l}\text { Other relaxation } \\
\text { practices }\end{array}$} \\
\hline $\begin{array}{l}\text { Movement } \\
\text { therapies (such as } \\
\text { Alexander } \\
\text { technique, Rolfing, } \\
\text { etc.) }\end{array}$ & & & & & & & \\
\hline
\end{tabular}

Please list any other Complementary Health Approaches you have used for pain, if any. Some examples include Ayurveda, healing touch/Reiki, and homeopathy. 
Please list any specific herbs/botanicals that you have used or intend to use for the treatment or prevention of pain. Some common examples include peppermint, black cohosh, lavender, kava kava, and ginger.

Please list any prescriptions you have used for pain, if any. If you use a pump or stimulator, please specify which one.

PLEASE CHECK THE BOX(ES) NEXT TO CONDITIONS YOU HAVE BEEN DIAGNOSED WITH:

\begin{tabular}{|c|c|c|}
\hline 口Hypertension & DChronic Bronchitis & 口Chronic Back Pain \\
\hline DHeart Disease & DDiabetes & DKnee Pain \\
\hline $\begin{array}{l}\text { Dinflammatory Bowel } \\
\text { Disease }\end{array}$ & $\begin{array}{l}\text { पCancer } \\
\text { ( } \mathrm{C} \text { Currently being treated) }\end{array}$ & DFibromyalgia \\
\hline $\begin{array}{l}\text { Dlmpaired Renal } \\
\text { Function/Kidney Disease }\end{array}$ & DStroke & DGout \\
\hline 口Asthma & DRheumatoid Arthritis & DMigraines \\
\hline DDepression & DOsteoarthritis & पTension Headaches \\
\hline DAnxiety & $\begin{array}{l}\text { DChronic Fatigue } \\
\text { Syndrome }\end{array}$ & $\begin{array}{l}\text { Dlnjury: Broken } \\
\text { Bone(s) }\end{array}$ \\
\hline DSpine and/or Neck Issues & $\begin{array}{l}\text { DTemporomandibular } \\
\text { Jaw Disorder (TMJ) }\end{array}$ & $\begin{array}{l}\text { Dinjury: } \\
\text { Musculoskeletal or Soft } \\
\text { Tissue Trauma (such } \\
\text { as whiplash, torn } \\
\text { ligament, etc.) }\end{array}$ \\
\hline
\end{tabular}

Please list any other health conditions you have. 


\section{PLEASE CHECK THE BOX WHICH BEST APPLIES TO YOU:}

What is your gender?
Male
- Female
口Other

What is your marital status?
Q Single
Married
Divorced
Separated
Widowed
What is your race/ethnicity?
W White/Caucasian
- Hispanic/Latino
African American
Native American or American Indian
Asian/Pacific Islander
Two or more races
Other

A member of an unmarried couple, living together

What is the highest degree or level of school you have completed?

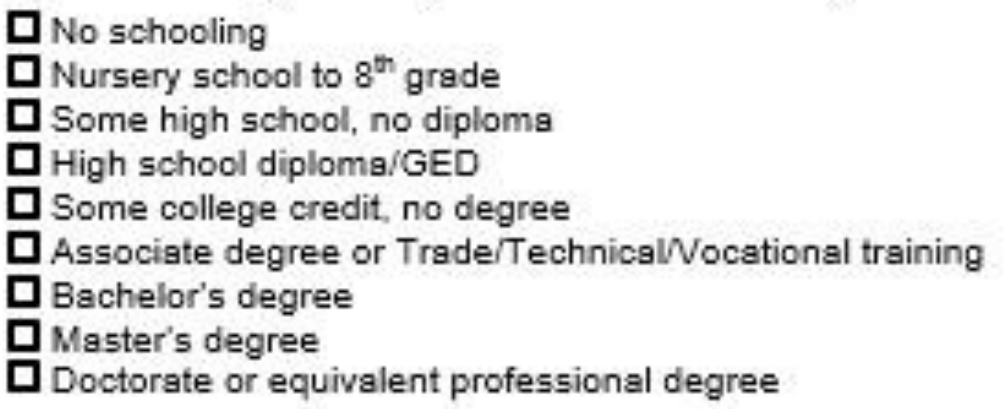

What is your work status?

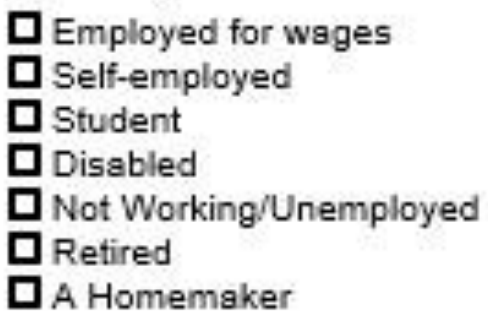


What is your military status?

口Veteran

Active duty

Guard/Reserves

- Served without Veteran Status (kicked out/dishonorably discharged)

Never served in the Armed Forces

What is your annual household income, from all sources?
Don't know
Less than $\$ 25,000$
口25,001 - $\$ 50,000$
口50,001-\$75,000
口 $\$ 75,001$ or more

What is your cigarette smoking status?

口Former Smoker

Current Smoker

I have never smoked

During the past month, did you participate in any physical activities or exercises such as yoga, aerobics, or walking for exercise?

口Yes

No

If yes, what type (for example, walking)?
If yes, how many days per week did you exercise?
If yes, how many minutes did you exercise per day?

$\bar{\square} \bar{\square}$

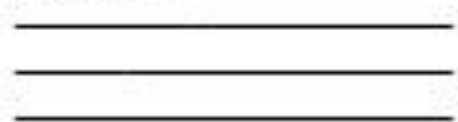

During the last 12 months, how often did you usually have any kind of drink containing alcohol? By a drink we mean half an ounce of absolute alcohol (e.g. a 12 ounce can or glass of beer or cooler, a 5 ounce glass of wine, or a drink containing 1 shot of liquor). Choose only one.

QEvery day

5-6 times a week

3-4 times a week

Twice a week

Once a week

2 to 3 times a month

3 to 11 times in the past year

1 or 2 times in the past year

None 
PLEASE FILL IN THE BLANK:

What is your age?

years
What is your weight?

lbs.

What is your current height?

If you are a person with a physical disability (of your legs). please state your lifetime maximum height. feet inches

Please place this form in the drop box located in the waiting room. Thank you for taking our survey? 


\section{Complete Listing of References}

1. Myers KA, Bello-Espinosa LE, Kherani A, Wei XC, Innes AM. TUBA1A Mutation Associated With Eye Abnormalities in Addition to Brain Malformation. Pediatr Neurol. 2015. doi: 10.1016/j.pediatrneurol.2015.07.004. PubMed PMID: 26294046.

2. Cauffield JS. The Psychoscoial aspects of complementary and alternative medicine. Pharmacotherapy. 2000;20(11):1289-94.

3. Manchikanti L, Singh V, Datta S, Cohen SP, Hirsch JA. American Society of Interventional Pain Physicians. Comprehensive review of epidemiology, scope, and impact of spinal pain. Pain Physician. 2009;12:E35-

70.

4.

Andersson HI. The epidemiology of chronic pain in a Swedish rural area. Qual Life Res. 1994;3:s19-s26.

5. Brattberg G, Thorslund M, Wikman A. The prevalence of pain in a general population. The results of a postal survey in a county of Sweden. Pain. 1989;37:215-22.

6. Wijnhoven HA, de Vet HC, Picavet HS. Explaining sex differences in chronoc musculoskeletal pain in a general population. Pain. 2006;124:158-66.

7. White KP, Harth M. Classification, epidemiology, and natural history of fibromyalgia. Current pain and headache reports. 2001;5(4):320-9. doi: 10.1007/s11916-001-0021-2.

8. Walitt B, Nahin RL, Katz RS, Bergman MJ, Wolfe F. The Prevalence and Characteristics of Fibromyalgia in the 2012 National Health Interview Survey. PLoS One. 2015;10(9):e0138024. doi: 10.1371/journal.pone.0138024. PubMed PMID: 26379048.

9. Clauw DJ. Fibromyalgia and related conditions. Mayo Clin Proc. 2015;90(5):680-92. doi: 10.1016/j.mayocp.2015.03.014. PubMed PMID: 25939940.

10. Baker K, Barkhuizen A. Pharmacologic treatment of fibromyalgia. Curr Pain Headache Rep. 2005;9(5):301-6. PubMed PMID: 16157056.

11. Administration FaD. Living with Fibromyalgia, Drugs Approved to Manage Pain Food and Drug Administration: Food and Drug Administration; 2013 [cited 2015 April 10]. Consumer Health Information]. Available from: http://www.fda.gov/downloads/ForConsumers/ConsumerUpdates/UCM107805.pdf.

12. Buskila D, Atzeni F, Sarzi-Puttini P. Etiology of fibromyalgia: The possible role of infection and vaccination. Special Issue on Infections Rheumatism and Autoimmunity. 2008;8(1):41-3. doi:

http://dx.doi.org.www.libproxy.wvu.edu/10.1016/j.autrev.2008.07.023.

13. NIH. Questions and Answers about Fibromyalgia 2014 [cited 2014 July]. Available from: http://www.niams.nih.gov/Health Info/Fibromyalgia/default.asp.

14. Bennett RM, Jones J, Turk DC, Russell IJ, Matallana L. An internet survey of 2,596 people with fibromyalgia. BMC musculoskeletal disorders. 2007;8:27. doi: 10.1186/1471-2474-8-27. PubMed PMID: 17349056; PubMed Central PMCID: PMCPMC1829161.

15. Arnold LM, Hudson JI, Hess EV, Ware AE, Fritz DA, Auchenbach MB, et al. Family study of fibromyalgia. Arthritis and rheumatism. 2004;50(3):944-52. Epub 2004/03/17. doi: 10.1002/art.20042. PubMed PMID: 15022338.

16. Clauw DJ, Crofford LJ. Chronic widespread pain and fibromyalgia: what we know, and what we need to know. Chronic Generalised Musculoskeletal Pain. 2003;17(4):685-701. doi:

http://dx.doi.org.www.libproxy.wvu.edu/10.1016/S1521-6942(03)00035-4.

17. Wolfe F, Clauw Dj Fau - Fitzcharles M-A, Fitzcharles Ma Fau - Goldenberg DL, Goldenberg Dl Fau - Hauser W, Hauser W Fau - Katz RS, Katz Rs Fau - Mease P, et al. Fibromyalgia criteria and severity scales for clinical and epidemiological studies: a modification of the ACR Preliminary Diagnostic Criteria for Fibromyalgia.

18. Fok E, Sandeman SR, Guildford AL, Martin YH. The Use of an IL-1 Receptor Antagonist Peptide to Control Inflammation in the Treatment of Corneal Limbal Epithelial Stem Cell Deficiency. Biomed Res Int. 2015;2015:516318. doi: 10.1155/2015/516318. PubMed PMID: 25705668; PubMed Central PMCID: PMC4330955.

19. Bjersing JL, Bokarewa MI, Mannerkorpi K. Profile of circulating microRNAs in fibromyalgia and their relation to symptom severity: an exploratory study. Rheumatol Int. 2015;35(4):635-42. doi: 10.1007/s00296-014-3139-3. PubMed PMID: 25261961.

20. Bjersing JL, Lundborg C, Bokarewa MI, Mannerkorpi K. Profile of cerebrospinal microRNAs in fibromyalgia. 
PLoS One. 2013;8(10):e78762. doi: 10.1371/journal.pone.0078762. PubMed PMID: 24205312; PubMed Central PMCID: PMCPMC3808359.

21. Ursini F, Naty S, Grembiale R. Fibromyalgia and obesity: the hidden link. Rheumatol Int. 2011;31(11):1403-8. doi: 10.1007/s00296-011-1885-z.

22. Giacomelli C, Talarico R, Bombardieri S, Bazzichi L. The interaction between autoimmune diseases and fibromyalgia: risk, disease course and management. Expert review of clinical immunology. 2013;9(11):1069-76. doi: 10.1586/1744666X.2013.849440. PubMed PMID: 24168413.

23. Staud R. Evidence for shared pain mechanisms in osteoarthritis, low back pain, and fibromyalgia. Current rheumatology reports. 2011;13(6):513-20. doi: 10.1007/s11926-011-0206-6. PubMed PMID: 21833699.

24. Wolfe F, Michaud K, Li T, Katz RS. Chronic conditions and health problems in rheumatic diseases: comparisons with rheumatoid arthritis, noninflammatory rheumatic disorders, systemic lupus erythematosus, and fibromyalgia. The Journal of rheumatology. 2010;37(2):305-15. doi: 10.3899/jrheum.090781. PubMed PMID: 20080915.

25. Ozgocmen S, Cimen OB, Ardicoglu O. Relationship between chest expansion and respiratory muscle strength in patients with primary fibromyalgia. Clinical rheumatology. 2002;21(1):19-22. PubMed PMID: 11954878.

26. Yang TY, Chen CS, Lin CL, Lin WM, Kuo CN, Kao CH. Risk for irritable bowel syndrome in fibromyalgia patients: a national database study. Medicine. 2015;94(10):e616. doi: 10.1097/MD.0000000000000616. PubMed PMID: 25761187.

27. Yanmaz MN, Mert M, Korkmaz M. The prevalence of fibromyalgia syndrome in a group of patients with diabetes mellitus. Rheumatol Int. 2012;32(4):871-4. doi: 10.1007/s00296-010-1618-8. PubMed PMID: 21221595.

28. Michaud K, Wolfe F. The association of rheumatoid arthritis and its treatment with sinus disease. The Journal of rheumatology. 2006;33(12):2412-5. PubMed PMID: 17143978.

29. Tietjen GE, Brandes JL, Peterlin BL, Eloff A, Dafer RM, Stein MR, et al. Allodynia in migraine: association with comorbid pain conditions. Headache. 2009;49(9):1333-44. doi: 10.1111/j.1526-4610.2009.01521.x. PubMed PMID: 19788473.

30. Devor M. - Neuropathic pain: what do we do with all these theories? 2001;- 45(- 9):- 1127.

31. Lipnik-Stangelj M. Mediators of inflammation as targets for chronic pain treatment. Mediators of inflammation. 2013;2013:783235. doi: 10.1155/2013/783235. PubMed PMID: 24347834; PubMed Central PMCID: PMC3848385.

32. Watkins LR, Milligan ED, Maier SF. Glial proinflammatory cytokines mediate exaggerated pain states: implications for clinical pain. Advances in experimental medicine and biology. 2003;521:1-21. PubMed PMID: 12617561.

33. Zhang J-M, An J. Cytokines, Inflammation and Pain. International anesthesiology clinics. 2007;45(2):27-37. doi: 10.1097/AIA.0b013e318034194e. PubMed PMID: PMC2785020.

34. FibroCenter. Fibromyalgia Pain Is Different: Pfizer; 2015 [cited 2015]. Available from:

http://www.fibrocenter.com/fibromyalgia-pain.aspx.

35. Maes M, Libbrecht I, Van Hunsel F, et al. The immune-inflammatory pathophysiology of fibromyalgia: increased serum soluble gp130, the common signal transducer protein of various neurotropic cytokines.

Psychoneuroendocrinology. 1999;24(4):371-83.

36. Wallace DJ L-IM, Hallegua D, Silverman S, Silver MH, Weisman MH. Cytokines play an aetiopathogenetic role in fibromyalgia: a hypothesis and pilot study. Rheumatology (Oxford). 2001;40(7):743-9.

37. Gur A KM, Erdogan S, Nas K, Cevik R, Sarac AJ. Regional cerebral flow and cytokines in young females with fibromyalgia. Clinical and experimental rheumatology JID - 8308521. 2002;20(6):753-60.

38. Gur A KM, Nas K, Remzi C, Denli A, Sarac J. Cytokines and depression in cases with fibromyalgia. Journal of Rheumatology. 2002;29(2):358-3561.

39. Hein G FS. Are advanced glycaton end-product-modified proteins of pathogentic importance in fibromyalgia. Rheumatology. 2002;41(10):1163-7.

40. Pache M OJ, Genth E, Mierau R, Kube T, Flammer J. Increased plasma endothilin-1 levels in fibromyalgia syndrome. Rheumatology. 2003;42(493-494).

41. Bagis S TL, Sahin G, et al. Free radicals and antioxidants in primary fibromyalgia: an oxidative stress disorder? Rheumatology international JID - 8206885.

42. Salemi S RJ, Wollina U, et al. Detection of interleukin 1 beta (IL-beta), IL0-6, and Tumor necrosis factor-alpha in skin of patients with fibromyalgia. Journal of Rheumatology. 2003;30(1):146-50. 
43. Kadetoff DF, Lampa JF, Westman MF, Andersson MF, Kosek E. Evidence of central inflammation in fibromyalgiaincreased cerebrospinal fluid interleukin-8 levels. 0328.

44. Xiao Y HW, Michalek JE, Russell IJ. Elevated serum high-sensitivity C-reactive protein levels in fibromyalgia syndrome patients correlate with body mass index, interleukin-6, interleukin-8, erythrocyte sedimentation rate. 2013. 45. Kaufmann IF, Schelling GF, Eisner CF, Fau RHP, Beyer AF, Krauseneck TF, et al. Decrease in adhesion molecules on polymorphonuclear leukocytes of patients with fibromyalgia. 0924.

46. Bazzichi LF, Rossi AF, Massimetti GF, Giannaccini GF, Giuliano T Fau DF, De Feo FF, et al. Cytokine patterns in fibromyalgia and their correlation with clinical manifestations. 0830.

47. Bazzichi L RA, Massimetti G, et al. Cytokine patterns in fibromyalgia and their correlation with clinical manifestations. Clin and Experim Rheum 2007;25(2):225-30.

48. Lund Haheim L, Nafstad P, Olsen I, Schwarze P R, KS. C-reactive protein variations for different chronic somatic disorders. 1001.

49. Rus A, Molina F, Gasso M, Camacho MV, Peinado MA, Moral ML. Nitric Oxide, Inflammation, Lipid Profile, and Cortisol in Normal- and Overweight Women With Fibromyalgia. Biol Res Nurs. 2015. doi: 10.1177/1099800415591035. PubMed PMID: 26134428.

50. Amel Kashipaz M, Swinden D, Todd I, Powell R. Normal production of inflammatory cytokines in chronic fatigue and fibromyalgia syndromes determined by intracellular cytokine staining in short-term cultured blood mononuclear cells. Clinical and experimental immunology. 2003;132(2):360-5. PubMed PMID: 12699429; PubMed Central PMCID: PMCPMC1808704.

51. Afsar B, Burucu R. Urinary albumin, protein excretion and circadian blood pressure in patients with fibromyalgia. Rheumatol Int. 2013;33:2391-8. doi: 10.1007/s00296-013-2748-6.

52. Sherman KJ, Innes KE. Yoga for metabolic risk factors: much ado about nothing or new form of adjunctive care? Journal of diabetes and its complications. 2014;28(3):253-4. doi: 10.1016/j.jdiacomp.2014.03.001. PubMed PMID: 24642263.

53. Bernardy K, Klose P, J BA, HS CE, Häuser W. : Cognitive behavioural therapies for fibromyalgia. : Cochrane Database of Systematic Reviews.

54. Andrew MR, Derry S, Aldington D, Cole P, J WP: Amitriptyline for neuropathic pain and fibromyalgia in adults: Cochrane Database of Systematic Reviews.

55. L RB, L WS, Buchbinder R. : Neuromodulators for pain management in rheumatoid arthritis. : Cochrane Database of Systematic Reviews.

56. Andrew MR, J WP, Derry S, Toelle T, C RAS. : Gabapentin for chronic neuropathic pain and fibromyalgia in adults: Cochrane Database of Systematic Reviews.

57. Roskell NS, Beard SM, Zhao Y, Le TK. A meta-analysis of pain response in the treatment of fibromyalgia. Pain practice: the official journal of World Institute of Pain. 2011;11(6):516-27. doi: 10.1111/j.1533-2500.2010.00441.x. PubMed PMID: 21199320.

58. PT LM, AC HR, J WP. : Duloxetine for treating painful neuropathy, chronic pain or fibromyalgia. : Cochrane Database of Systematic Reviews.

59. Häuser W, Urrútia G, Tort S, Üçeyler N, Walitt B: Serotonin and noradrenaline reuptake inhibitors (SNRIs) for fibromyalgia syndrome. : Cochrane Database of Systematic Reviews.

60. Derry S, Gill D, Phillips T, Andrew MR. : Milnacipran for neuropathic pain and fibromyalgia in adults. : Cochrane Database of Systematic Reviews.

61. J WP, Derry S, Andrew MR, Aldington D, Cole P, C RAS, et al.: Antiepileptic drugs for neuropathic pain and fibromyalgia - an overview of Cochrane reviews. : Cochrane Database of Systematic Reviews.

62. Lanza FL, Chan FK, Quigley EM, Practice Parameters Committee of the American College of G. Guidelines for prevention of NSAID-related ulcer complications. The American journal of gastroenterology. 2009;104(3):728-38. doi: 10.1038/ajg.2009.115. PubMed PMID: 19240698.

63. Zhao Y, Sun P, Watson P, Mitchell B, Swindle R. Comparison of medication adherence and healthcare costs between duloxetine and pregabalin initiators among patients with fibromyalgia. Pain practice : the official journal of World Institute of Pain. 2011;11(3):204-16. doi: 10.1111/j.1533-2500.2010.00412.x. PubMed PMID: 20807351.

64. Busse JW, Ebrahim S, Connell G, Coomes EA, Bruno P, Malik K, et al. Systematic review and network metaanalysis of interventions for fibromyalgia: a protocol. Systematic reviews. 2013;2:18. doi: 10.1186/2046-4053-2-18.

PubMed PMID: 23497523; PubMed Central PMCID: PMC3610251. 
65. Noller V, Sprott H. Prospective epidemiological observations on the course of the disease in fibromyalgia patients. Journal of negative results in biomedicine. 2003;2:4. doi: 10.1186/1477-5751-2-4. PubMed PMID: 12969513; PubMed Central PMCID: PMC194775.

66. Reid S, Whooley D, Crayford T, Hotopf M. Medically unexplained symptoms--GPs' attitudes towards their cause and management. Family practice. 2001;18(5):519-23. PubMed PMID: 11604375.

67. Birse F, Derry S, Andrew MR. : Phenytoin for neuropathic pain and fibromyalgia in adults. : Cochrane Database of Systematic Reviews.

68. Corrigan R, Derry S, J WP, Andrew MR. : Clonazepam for neuropathic pain and fibromyalgia in adults. : Cochrane Database of Systematic Reviews.

69. J WP, Derry S, Andrew MR, A KE. : Carbamazepine for chronic neuropathic pain and fibromyalgia in adults. : Cochrane Database of Systematic Reviews.

70. J WP, Derry S, PT LM, Andrew MR. : Topiramate for neuropathic pain and fibromyalgia in adults. : Cochrane Database of Systematic Reviews.

71. Tort S, Urrútia G, Nishishinya María B, Walitt B. : Monoamine oxidase inhibitors (MAOIs) for fibromyalgia syndrome. : Cochrane Database of Systematic Reviews.

72. Gill D, Derry S, J WP, Andrew MR. : Valproic acid and sodium valproate for neuropathic pain and fibromyalgia in adults. : Cochrane Database of Systematic Reviews.

73. J WP, Derry S, Andrew MR. : Lamotrigine for chronic neuropathic pain and fibromyalgia in adults. : Cochrane Database of Systematic Reviews.

74. Hearn L, Derry S, Andrew MR. : Lacosamide for neuropathic pain and fibromyalgia in adults. : Cochrane Database of Systematic Reviews.

75. Gaskell H, Andrew MR, Derry S, Stannard C. : Oxycodone for neuropathic pain and fibromyalgia in adults. : Cochrane Database of Systematic Reviews.

76. Rheumatology ACo. Patient Resources: Diseases and Conditions - Fibromyalgia: American College of Rheumatology; 2013 [cited 2015 April 10]. Available from:

http://www.rheumatology.org/Practice/Clinical/Patients/Diseases_And_Conditions/Fibromyalgia/.

77. Ernst E. Oxford handbook of complementary medicine. Oxford ; New York: Oxford University Press; 2008. xxiii, 424 p. p.

78. Schoonees A, Visser J, Musekiwa A, Volmink J. : Pycnogenol® (extract of French maritime pine bark) for the treatment of chronic disorders. : Cochrane Database of Systematic Reviews.

79. Plaganyi EE, van Putten I, Thebaud O, Hobday AJ, Innes J, Lim-Camacho L, et al. A quantitative metric to identify critical elements within seafood supply networks. PLoS One. 2014;9(3):e91833. doi: 10.1371/journal.pone.0091833. PubMed PMID: 24633147; PubMed Central PMCID: PMCPMC3954797.

80. Scheuermeyer FX, Wong H, Yu E, Boychuk B, Innes G, Grafstein E, et al. Development and validation of a prediction rule for early discharge of low-risk emergency department patients with potential ischemic chest pain. CJEM. 2014;16(2):106-19. PubMed PMID: 24626115.

81. Santamaria RM, Innes NP, Machiulskiene V, Evans DJ, Alkilzy M, Splieth CH. Acceptability of different caries management methods for primary molars in a RCT. Int J Paediatr Dent. 2015;25(1):9-17. doi: 10.1111/ipd.12097. PubMed PMID: 24602167.

82. Adams D, Wu T, Yang X, Tai S, Vohra S. : Traditional Chinese medicinal herbs for the treatment of idiopathic chronic fatigue and chronic fatigue syndrome. : Cochrane Database of Systematic Reviews.

83. Painter JT, Crofford LJ. Chronic opioid use in fibromyalgia syndrome: a clinical review. Journal of clinical rheumatology : practical reports on rheumatic \& musculoskeletal diseases. 2013;19(2):72-7. doi: 10.1097/RHU.0b013e3182863447. PubMed PMID: 23364665.

84. CDC. Drug-poisoning Deaths Involving Opioid Analgesics: United States, 1999-2011: CDC; 2014 [cited 2015 4/17]. Available from: http://www.cdc.gov/nchs/data/databriefs/db166.htm.

85. Williams DA, Clauw DJ. Understanding fibromyalgia: lessons from the broader pain research community. The journal of pain : official journal of the American Pain Society. 2009;10(8):777-91. doi: 10.1016/j.jpain.2009.06.001. PubMed PMID: 19638325; PubMed Central PMCID: PMC2741022.

86. O'Neil M, Hannah KL. Understanding the cultures of Prescription Drug Abuse, Misuse, Addiction, and Diversion. WV Medical Journal. 2010;106(4):64-70. 
87. Painter JT, Crofford LJ, Talbert J. Geographic variation of chronic opioid use in fibromyalgia. Clin Ther. 2013;35(3):303-11. doi: 10.1016/j.clinthera.2013.02.003. PubMed PMID: 23485077; PubMed Central PMCID: PMC4346177.

88. Rosenberg EI, Genao I, Chen I, Mechaber AJ, Wood JA, Faselis CJ, et al. Complementary and alternative medicine use by primary care patients with chronic pain. Pain Med. 2008;9(8):1065-72. doi: 10.1111/j.15264637.2008.00477.x. PubMed PMID: 18564996.

89. Astin J. Why patients use alternative medicine: Results of a national survey. JAMA. 1998;279(19):1548-53.

90. Eisenberg DM, Kessler RC, Foster C, Norlock FE, Calkins DR, Delbanco TL. Unconventional medicine in the United States: Prevalence, costs, and patterns of use. New England Journal of Medicine. 1993;328:246-52.

91. Eisenberg DM, Kessler RC, Van Rompay MI, Kaptchuk TJ, Wilkey SA, Appel S, et al. Perceptions about complementary therapies relative to conventional therapies among adults who use both: Results from a national survey. Ann Intern Med. 2001;135:344-51.

92. Barret B, Marchand L, Scheder J, Appelbaum D, Plane MB, Blustein J, et al. What complementary and alternative medicine practitioners say about health and health care. Ann Fam Med. 2004;2:253-9.

93. Fleming S RDPMMPFMF. CAM therapies among primary care patients using opioid therapy for chronic pain. BMC Complementary and Alternative Medicine. 2007;7(15).

94. Sherman K.J et al. Complementary and alternative medical therapies for chronic low back pain: what treatments are patients willing to try? BMC Complementary and Alternative Medicine. 2004;4(9).

95. Rosenberg Ei et al. Complementary and Alternative Medicine Use by Primary Care Patients with Chronic pain. Pain medicine (Malden, Mass) JID - 100894201. 2008;9(8):1065-72.

96. Druss BG, Rosenheck RA. Association between use of unconventional therapies and conventional medical services. JAMA. 1999;282(7):651-6. PubMed PMID: 10517718.

97. Rao JK, Mihaliak K, Kroenke K, et al. Use of complimentary therapies for arthrtitis among patients of rheumatologists. Ann Intern Med. 1999;131(6):409-16.

98. Brown CM. Use of Alternative therapies and their impact on compliance: perceptions of community pharmacists in Texas. J Am Pharm Assoc. 1998;38(5):603-8.

99. Berman BM, Singh BB, Hartnoll SM, Singh BK, Reilly D. Primary care physicians and complementary/alternative medicine: Training, attitudes and practice patterns. J Am Board Fam Pract. 1998;11:272-81. 100. Wagner PJ, Jester D, LeClair B, et al. Taking the edge off: why patients choose St. John's Wort. J Fam Pract. 1999;48(8):615-9.

101. Sollner W, Maislinger S, DeVries A, et al. Use of complementary and alternative medicine by cancer patients is not associated with perceived distress or poor compliance with standard treatment but with active coping behavior: A survey. Cancer. 2000;89(4):873-80.

102. Palinkas LA, Kabongo ML. San Diego Unified Practice in Family Medicine. The use of complementary and alternative medicine by primary care patients. A SURF*NET study. J Fam Pract. 2000;49(12):1221-130.

103. Kanning M. Complementary and alternative therapies for rheumatic diseases I. Part 1. Issues. Why I would want to use complementary and alternative therapy: A patient's perspective. Rheum Dis Clin North America. 1999;25(4):82330.

104. NIH. Complementary, Alternative, or Integrative Health: What's In a Name? Bethesda, MD2015 [cited 2015 4/13]. Available from: https://nccih.nih.gov/health/integrative-health.

105. Braverman DL, Ericken JJ, Shah RV, Franklin DJ. Interventions in chronic pain management 3. New frontiers in pain management: complementary techniques. Arch Phys Med Rehabil. 2003;84(3 Suppl 1):S45-9.

106. Eisenberg DM, Davis RB, Ettner SL, Appel S, Wilkey S, Van Rompay M, et al. Trends in alternative medicine use in the United States, 1990-1997: results of a follow-up of a national survey. JAMA. 1998;280:1569-75.

107. Institute of Medicine (U.S.). Committee on the Use of Complementary and Alternative Medicine by the American Public. Complementary and alternative medicine in the United States. Washington, DC: National Academies Press; 2005. xx, 337 p. p.

108. Kirkland J, Matthews, Holly F., Sulivan III, C.W., Baldwin, Karen. Herbal and Magical Medicine: Traditional Healing Today: Duke University Press; 1992.

109. Nahin RL, Barnes PM, Stussman BJ, Bloom B. Costs of complementary and alternative medicine (CAM) and frequency of visits to CAM practitioners: United States, 2007. National health statistics reports. 2009(18):1-14. PubMed PMID: 19771719.

110. Barnes PM, Powell-Griner E, McFann K, Nahin RL. Complementary and alternative medicine use among adults: 
United States 2002. Hyattsville, MD: National Center for Health Statistics, 2004 Contract No.: Report.

111. Arcury Q, Bell \& Vitolins. Complementary and alternativemedicine use among rural adults. Complementary Health Practice Review. 2002;7(3):167-86.

112. Bausell RB, Lee WL, Berman BM. Demographic and health-related correlates to visits to complementary and alternative medical providers. Medical care. 2001;39(2):190-6. PubMed PMID: 11176556.

113. Graham RE, Ahn AC, Davis RB, O'Connor BB, Eisenberg DM, Phillips RS. Use of complementary and alternative medical therapies among racial and ethnic minority adults: results from the 2002 National Health Interview Survey. J Natl Med Assoc. 2005;97(4):535-45. PubMed PMID: 15868773; PubMed Central PMCID: PMC2568705. 114.

Montalto CP, Bhargava, V., \& Hong, G. S. Use of complementary and alternative medicineby older adults: An exploratory study. Complementary Health Practice Review. 2006;11(1):27-46.

115. Tindle HA, Davis RB, Phillips RS, Eisenberg DM. Trends in use of complementary and alternative medicine by US adults: 1997-2002. Altern Ther Health Med. 2005;11(1):42-9. PubMed PMID: 15712765.

116. Bhargava V, Hong GS, Montalto CP. Use of practitioner-based and self-care complementary and alternative medicine in the united states: A demand for health perspective. Family and Consumer Sciences Research Journal. 2012;41(1):18-35. doi: 10.1111/j.1552-3934.2012.02126.x.

117. Schuster TL, Dobson M, Jauregui M, Blanks RH. Wellness lifestyles I: A theoretical framework linking wellness, health lifestyles, and complementary and alternative medicine. J Altern Complement Med. 2004;10(2):349-56. doi: 10.1089/107555304323062347. PubMed PMID: 15165416.

118. Davis MA, West AN, Weeks WB, Sirovich BE. Health Behaviors and Utilization among Users of Complementary and Alternative Medicine for Treatment versus Health Promotion. Health services research.

2011;46(5):1402-16. doi: 10.1111/j.1475-6773.2011.01270.x.

119. Green J. The herbal medicine-makers' handbook : a home manual. Freedom, Calif.: The Crossing Press; 2000. 384 p. p.

120. Cech R. [Growing medicinal plants]. Williams, OR: Horizon Herbs; 1995. v. <1-7, 9, $11>$ p.

121. Amjad H. Folk Medicine of Appalachia: A Vanishing Tradition: lulu.com (self-published); 2006. 340 p.

122. Rehder JB. Appalachian folkways. Baltimore: Johns Hopkins University Press; 2004. x, 353 p. p.

123. Audette JF, Bailey A. Integrative pain medicine : the science and practice of complementary and alternative medicine in pain management. Totowa, N.J.: Humana; 2008. xiv, 579 p. p.

124. Cavender A. Folk Medicine in Southern Appalachia: The University of North Carolina Press; 2003. 266 p.

125. Wu CH, Wang CC, Tsai MT, Huang WT, Kennedy J. Trend and pattern of herb and supplement use in the United States: results from the 2002, 2007, and 2012 national health interview surveys. Evidence-based complementary and alternative medicine : eCAM. 2014;2014:872320. doi: 10.1155/2014/872320. PubMed PMID: 25574184; PubMed Central PMCID: PMC4276694.

126. Kessler RC, Davis RB, Foster DF, Van Rompay MI, Walters EE, Wilkey SA, et al. Long-term trends in the use of complementary and alternative medical therapies in the United States. Ann Intern Med. 2001;135(4):262-8. Epub 2001/08/21. PubMed PMID: 11511141.

127. Kennedy J. Herb and supplement use in the US adult population. Clin Ther. 2005;27(11):1847-58. doi: 10.1016/j.clinthera.2005.11.004. PubMed PMID: 16368456.

128. Schaffer DM, Gordon NP, Jensen CD, Avins AL. Nonvitamin, nonmineral supplement use over a 12-month period by adult members of a large health maintenance organization. J Am Diet Assoc. 2003;103(11):1500-5. Epub 2003/10/25. doi: 10.1016/s0002. PubMed PMID: 14576716.

129. Kelly JP, Kaufman DW, Kelley K, Rosenberg L, Mitchell AA. Use of herbal/natural supplements according to racial/ethnic group. J Altern Complement Med. 2006;12(6):555-61. Epub 2006/08/04. doi: 10.1089/acm.2006.12.555. PubMed PMID: 16884347.

130. Tanaka MJ, Gryzlak BM, Zimmerman MB, Nisly NL, Wallace RB. Patterns of natural herb use by Asian and Pacific Islanders. Ethn Health. 2008;13(2):93-108. Epub 2008/04/22. doi: 10.1080/13557850701830349. PubMed PMID: 18425709.

131. Dailey RK, Neale AV, Northrup J, West P, Schwartz KL. Herbal product use and menopause symptom relief in primary care patients: a MetroNet study. Journal of women's health. 2003;12(7):633-41. Epub 2003/10/30. doi: 10.1089/154099903322404285. PubMed PMID: 14583104.

132. Mackenzie ER, Taylor L, Bloom BS, Hufford DJ, Johnson JC. Ethnic minority use of complementary and alternative medicine (CAM): a national probability survey of CAM utilizers. Altern Ther Health Med. 2003;9(4):50-6. 
Epub 2003/07/19. PubMed PMID: 12868252.

133. Hung OL, Shih RD, Chiang WK, Nelson LS, Hoffman RS, Goldfrank LR. Herbal preparation use among urban emergency department patients. Acad Emerg Med. 1997;4(3):209-13. Epub 1997/03/01. PubMed PMID: 9063549. 134. Klepser TB, Doucette WR, Horton MR, Buys LM, Ernst ME, Ford JK, et al. Assessment of patients' perceptions and beliefs regarding herbal therapies. Pharmacotherapy. 2000;20(1):83-7. Epub 2000/01/21. PubMed PMID: 10641978. 135. Harnack LJ, Rydell SA, Stang J. Prevalence of use of herbal products by adults in the Minneapolis/St Paul, Minn, metropolitan area. Mayo Clin Proc. 2001;76(7):688-94. Epub 2001/07/11. doi: 10.4065/76.7.688. PubMed PMID: 11444400.

136. Bair YA, Gold EB, Greendale GA, Sternfeld B, Adler SR, Azari R, et al. Ethnic differences in use of complementary and alternative medicine at midlife: longitudinal results from SWAN participants. Am J Public Health. 2002;92(11):1832-40. Epub 2002/10/31. PubMed PMID: 12406817; PubMed Central PMCID: PMCPMC1447337.

137. Sawni A, Thomas R. Pediatricians' attitudes, experience and referral patterns regarding Complementary/Alternative Medicine: a national survey. BMC Complement Altern Med. 2007;7:18. doi: 10.1186/14726882-7-18. PubMed PMID: 17547752; PubMed Central PMCID: PMC1894987.

138. Tom Xu K, Farrell TW. The complementarity and substitution between unconventional and mainstream medicine among racial and ethnic groups in the United States. Health Serv Res. 2007;42(2):811-26. Epub 2007/03/17. doi: 10.1111/j.1475-6773.2006.00628.x. PubMed PMID: 17362219; PubMed Central PMCID: PMCPMC1955362.

139. Chao MT, Wade C, Kronenberg F. Disclosure of complementary and alternative medicine to conventional medical providers: Variation by race/ethnicity and type of CAM. Journal of the National Medical Association. 2008;100(11):1341-9.

140. Upchurch DM, Chyu L, Greendale GA, Utts J, Bair YA, Zhang G, et al. Complementary and alternative medicine use among American women: Findings from the National Health Interview Survey, 2002. Journal of women's health. 2007;16(1):102-13. doi: 10.1089/jwh.2006.M074.

141. Blalock SJGPJPRANLLCLFJJM. Factors Associated with Potential Medication-Herb/natural Product Interactions in a Rural Community. Alternative Therapies in Health \& Medicine. 2009;15(5):26-34.

142. Nguyen LT, Davis RB, Kaptchuk TJ, Phillips RS. Use of complementary and alternative medicine and self-rated health status: Results from a national survey. Journal of General Internal Medicine. 2011;26(4):399-404. doi: 10.1007/s11606-010-1542-3.

143. Nguyen HT, Grzywacz JG, Lang W, Walkup M, Arcury TA. Effects of Complementary Therapy on Health in a National U.S. Sample of Older Adults. Journal of Alternative \& Complementary Medicine. 2010;16(7):701-6. doi: 10.1089/acm.2009.0442.

144. Neiberg RH, Aickin M, Grzywacz JG, Lang W, Quandt SA, Bell RA, et al. Occurrence and co-occurrence of types of complementary and alternative medicine use by age, gender, ethnicity, and education among adults in the United States: The 2002 national health interview survey (NHIS). Journal of Alternative and Complementary Medicine. 2011;17(4):363-70. doi: 10.1089/acm.2009.0157.

145. Nahin RL, Dahlhamer JM, Taylor BL, Barnes PM, Stussman BJ, Simile CM, et al. Health behaviors and risk factors in those who use complementary and alternative medicine. BMC Public Health. 2007;7:217. doi: 10.1186/147124587-217. PubMed PMID: 17723149; PubMed Central PMCID: PMC2031902.

146. Nahin RL, Dahlhamer JM, Stussman BJ. Health need and the use of alternative medicine among adults who do not use conventional medicine. BMC Health Serv Res. 2010;10:220. doi: 10.1186/1472-6963-10-220. PubMed PMID: 20670418; PubMed Central PMCID: PMC2919531.

147. Misra R, Balagopal P, Klatt M, Geraghty M. Complementary and alternative medicine use among Asian Indians in the United States: A national study. Journal of Alternative and Complementary Medicine. 2010;16(8):843-52. doi: 10.1089/acm.2009.0517.

148. Mehta DH, Phillips RS, Davis RB, McCarthy EP. Use of complementary and alternative therapies by Asian Americans. Results from the National Health Interview Survey. Journal of General Internal Medicine. 2007;22(6):762-7. doi: 10.1007/s11606-007-0166-8.

149. Kennedy J, Wang CC, Wu CH. Patient Disclosure about Herb and Supplement Use among Adults in the US. Evidence-based complementary and alternative medicine : eCAM. 2008;5(4):451-6. doi: 10.1093/ecam/nem045. PubMed PMID: 18955213; PubMed Central PMCID: PMC2586312. 
150. Kannan VD, Gaydos LM, Atherly AJ, Druss BG. Medical utilization among wellness consumers. Med Care Res Rev. 2010;67(6):722-36. Epub 2010/06/04. doi: 10.1177/1077558710370706. PubMed PMID: 20519427.

151. Jones L, Sciamanna C, Lehman E. Are those who use specific complementary and alternative medicine therapies less likely to be immunized? Preventive medicine. 2010;50(3):148-54. doi: 10.1016/j.ypmed.2009.12.001.

152. Barner JC, Bohman TM, Brown CM, Richards KM. Use of complementary and alternative medicine for treatment among African-Americans: A multivariate analysis. Research in Social and Administrative Pharmacy. 2010;6(3):196-208. doi: 10.1016/j.sapharm.2009.08.001.

153. Hildreth KD EC. Alternative worldviews and the utilization of conventional and complementary medicine. Sociological Inquiry. 2007;77(1):76-103.

154. Birdee GS, Phillips RS, Davis RB, Gardiner P. Factors associated with pediatric use of complementary and alternative medicine. Pediatrics. 2010;125(2):249-56. doi: 10.1542/peds.2009-1406.

155. Grzywacz JG, Lang W, Suerken C, Quandt SA, Bell RA, Arcury TA. Age, race, and ethnicity in the use of complementary and alternative medicine for health self-management: evidence from the 2002 National Health Interview Survey. Journal of aging and health. 2005;17(5):547-72. Epub 2005/09/24. doi: 10.1177/0898264305279821. PubMed PMID: 16177450.

156. Grzywacz JG, Suerken CK, Neiberg RH, Lang W, Bell RA, Quandt SA, et al. Age, ethnicity, and use of complementary and alternative medicine in health self-management. Journal of health and social behavior. 2007;48(1):8498. PubMed PMID: 17476925.

157. Gardiner PRATRAACEDMPRS. Factors Associated with Herbal Therapy use by Adults in the United States. Alternative Therapies in Health \& Medicine. 2007;13(2):22-9.

158. Elmer GW, Lafferty WE, Tyree PT, Lind BK. Potential interactions between complementary/alternative products and conventional medicines in a Medicare population. Ann Pharmacother. 2007;41(10):1617-24. Epub 2007/09/06. doi: 10.1345/aph.1K221. PubMed PMID: 17785609; PubMed Central PMCID: PMCPMC2864004.

159. Ellison CG, Bradshaw M, Roberts CA. Spiritual and religious identities predict the use of complementary and alternative medicine among US adults. Preventive medicine. 2012;54(1):9-12. doi: 10.1016/j.ypmed.2011.08.029.

160. Upchurch DM, Dye CE, Chyu L, Gold EB, Greendale GA. Demographic, behavioral, and health correlates of complementary and alternative medicine and prayer use among midlife women: 2002. Journal of women's health. 2010;19(1):23-30. doi: 10.1089/jwh.2008.1096. PubMed PMID: 20088655; PubMed Central PMCID: PMC2828262.

161. Barnes PM, Bloom B, Nahin RL. Complementary and alternative medicine use among adults and children: United States, 2007. National health statistics reports. 2008(12):1-23. Epub 2009/04/14. PubMed PMID: 19361005.

162. Bardia A, Nisly NL, Zimmerman MB, Gryzlak BM, Wallace RB. Use of herbs among adults based on evidence based indications: findings from the National Health Interview Survey. Mayo Clin Proc. 2007;82(5):561-6. Epub 2007/05/12. doi: 10.4065/82.5.561. PubMed PMID: 17493422; PubMed Central PMCID: PMCPMC1964882.

163. Arcury TA, Grzywacz JG, Bell RA, Neiberg RJ, Lang W, Quandt SA. Herbal remedy use as health selfmanagement among older adults. Journals of Gerontology: Series B: Psychological Sciences and Social Sciences. 2007;62B(2):S142-S9.

164. Brown CM, Barner JC, Richards KM, Bohman TM. Patterns of complementary and alternative medicine use in African Americans. Journal of Alternative and Complementary Medicine. 2007;13(7):751-8. doi: 10.1089/acm.2006.6392.

165. Mehta DH, Gardiner PM, Phillips RS, McCarthy EP. Herbal and dietary supplement disclosure to health care providers by individuals with chronic conditions. J Altern Complement Med. 2008;14(10):1263-9. Epub 2008/11/27. doi:

10.1089/acm.2008.0290. PubMed PMID: 19032071; PubMed Central PMCID: PMCPMC2787410.

166. Clarke TC, Black LI, Stussman BJ, Barnes PM, Nahin RL. Trends in the use of complementary health approaches among adults: United States, 2002-2012. National health statistics reports. 2015(79):1-16. PubMed PMID: 25671660.

167. Commission AR. The Appalachian Region 2015 [cited 2015]. Available from:

http://www.arc.gov/appalachian_region/TheAppalachianRegion.asp.

168. Crellin JK, Philpott J, Crellin JK. A reference guide to medicinal plants : herbal medicine past and present.

Durham, NC: Duke University Press; 1997. 551 p. p.

169. Crellin JK, Bass ALT, Philpott J. Trying to give ease : Tommie Bass and the story of herbal medicine. Durham: Duke University Press; 1997. xii. 335 p. p.

170. Allen JL. Through Cumberland Gap on Horseback. Harper's Magazine. June 1886:50-66.

171. Koehler JH. Ginseng and goldenseal growers' handbook. Wausau, Wis.,: P. F. Stolze; 1912. vii, 116 p. p. 
172. Rago LO. Blackberry Cove herbal : healing with common herbs in the Appalachian wise-woman tradition. Sterling, Va.: Capital Books; 2000. ix, 166 p. p.

173. Growing \& Marketing Ginseng, Goldenseal \& Other Woodland Medicinals. North Carolina Libraries (Online). 2006;64(1/2):35-. PubMed PMID: 21528280.

174. Persons WS, Davis JM. Growing \& marketing ginseng, goldenseal \& other woodland medicinals. Fairview, N.C.: Bright Mountain Books; 2005. xii, 466 p. p.

175. Atkins ABS. "She Didn't Go Sangin' Alone!". Goldenseal. 1999:27-9.

176. Amjad H. Life \& Thymes of an Appalachian Herbalist: lulu.com (self-published); 2006.

177. Flynn MHJ. Tending the Commons: Folklife and Landscape in Southern West Virginia1993. Available from:

http://www.loc.gov/collections/folklife-and-landscape-in-southern-west-virginia/articles-and-essays/americanginsenghttp://www.loc.gov/collections/folklife-and-landscape-in-southern-west-virginia/articles-and-essays/americanginseng-and-the-idea-of-the-commons/and-the-idea-of-the-commons/.

178. Goodwin JG. Going 'Senging. Goldenseal. 1985:16-21.

179. Bolyard JL. Medicinal plants and home remedies of Appalachia. Springfield, Ill.: Thomas; 1981. xvii, 187 p. p. 180.

Collins KC, Hunter L. Foxfire 11 : the old homeplace, wild plant uses, preserving and cooking food, hunting stories, fishing, and more affairs of plain living. 1st ed. New York: Anchor Books; 1999. xi, 313 p. p.

181. Moerman DE. Native American Medicinal Plants: An Ethnobotanical Dictionary. First Abridged Edition ed. Portland, OR: Timber Press, Inc.; 2009. 799 p.

182. Wilson CR, Ferris WR. Encyclopedia of Southern culture. Chapel Hill: University of North Carolina Press; 1989. xxi, 1634 p. p.

183. Cook C, Baisden D. Ancillary Use of Folk Medicine by Patients in Primary Care Clinics in Southwestern WestVirginia. Southern Med J. 1986;79(9):1098-101. PubMed PMID: WOS:A1986E085900013.

184. Glover DD, Amonkar M, Rybeck BF, Tracy TS. Prescription, over-the-counter, and herbal medicine use in a rural, obstetric population. American journal of obstetrics and gynecology. 2003;188(4):1039-45. doi:

http://dx.doi.org/10.1067/mob.2003.223.

185. Cool R. Folk Medicine in the Kentucky Hills. Appalachian Heritage. 1976:51-6.

186. Ward B. Folk Medicine: West Virginia Humanities Council; 2010 [cited 2015 April 21]. Available from: http://www.wvencyclopedia.org/articles/2221.

187. Deskins C. Healing from the Hills. Goldenseal. 1990:60-4.

188. Tanner L. Clay County Folklore and Folk Medicine: Empty Rockers \& Sassafras Tea. Goldenseal. 1995:67-9. 189. Ackers-Johnson M, Talasila A, Sage AP, Long X, Bot I, Morrell NW, et al. Myocardin regulates vascular smooth muscle cell inflammatory activation and disease. Arteriosclerosis, thrombosis, and vascular biology. 2015;35(4):81728. doi: 10.1161/ATVBAHA.114.305218. PubMed PMID: 25614278.

190. Seaton CT. Hippie homesteaders : arts, crafts, music, and living on the land in West Virginia. First Edition. ed. Morgantown, West Virginia: West Virginia University Press; 2014. xiii, 281 pages p.

191. Bennett TGaA. Catfish: Portrait of an Herb Doctor. Goldenseal. 1977:46-51.

192. Ernst E. Usage of complementary therapies in rheumatology: a systematic review. Clinical rheumatology. 1998;17(4):301-5. PubMed PMID: 9776112.

193. Pullar T, Capell HA, Millar A, Brooks RG. Alternative medicine: cost and subjective benefit in rheumatoid arthritis. British medical journal. 1982;285(6355):1629-31. PubMed PMID: 6814682; PubMed Central PMCID: PMC1500773.

194. Ernst E, White A. The BBC survey of complementary medicine use in the UK. Complementary therapies in medicine. 2000;8(1):32-6. doi: http://dx.doi.org/10.1016/S0965-2299(00)90833-1.

195. Higham C, Ashcroft C, Jayson MI. Non-prescribed treatments in rheumatic diseases. The Practitioner. 1983;227(1381):1201-5. PubMed PMID: 6604270.

196. Dimmock S, Troughton PR, Bird HA. Factors predisposing to the resort of complementary therapies in patients with fibromyalgia. Clinical rheumatology. 1996;15(5):478-82. PubMed PMID: 8894361.

197. Shaver JL, Wilbur J, Lee H, Robinson FP, Wang E. Self-reported medication and herb/supplement use by women with and without fibromyalgia. Journal of women's health. 2009;18(5):709-16. doi: 10.1089/jwh.2008.1194. PubMed PMID: 19445618.

198. A Blueprint for Transforming 
Prevention, Care, Education, and Research. Washington, DC: Institute of

Medicine, 2012 June 2011. Report No.

199. Mason L, Moore RA, Derry S, Edwards JE, McQuay HJ. Systematic review of topical capsaicin for the treatment of chronic pain. BMJ. 2004;328(7446):991. doi: 10.1136/bmj.38042.506748.EE. PubMed PMID: 15033881; PubMed Central PMCID: PMC404499.

200. Miyasaka Lincoln S, N AÁ, Soares B. : Passiflora for anxiety disorder. : Cochrane Database of Systematic Reviews.

201. Miyasaka Lincoln S, N AÁ, Soares B. : Valerian for anxiety disorders. : Cochrane Database of Systematic Reviews.

202. H PM, Ernst E. : Kava extract versus placebo for treating anxiety. : Cochrane Database of Systematic Reviews. 203.

Cui X, Trinh K, Wang Y-J. : Chinese herbal medicine for chronic neck pain due to cervical degenerative disc disease. : Cochrane Database of Systematic Reviews.

204. Pittler MH, Ernst E. Feverfew for preventing migraine. The Cochrane database of systematic reviews.

2004(1):CD002286. doi: 10.1002/14651858.CD002286.pub2. PubMed PMID: 14973986.

205. Wider B, Pittler MH, Ernst E. Feverfew for preventing migraine. The Cochrane database of systematic reviews. 2015;4:CD002286. doi: 10.1002/14651858.CD002286.pub3. PubMed PMID: 25892430.

206. Wider B, H PM, Ernst E. : Feverfew for preventing migraine. : Cochrane Database of Systematic Reviews.

207. Linde K, M BM, Kriston L. : St John's wort for major depression. : Cochrane Database of Systematic Reviews. 208.

Oltean H, Robbins C, W vTM, M BB, Bombardier C, J GJ. : Herbal medicine for low-back pain. : Cochrane Database of Systematic Reviews.

209. Liu Jian P, Yang M, Liu Y, Wei Mao L, Grimsgaard S. : Herbal medicines for treatment of irritable bowel syndrome. : Cochrane Database of Systematic Reviews.

210. Cameron M, J GJ, Chrubasik S. : Herbal therapy for treating rheumatoid arthritis. : Cochrane Database of Systematic Reviews.

211. Cabrera C. Fibromyalgia : a journey toward healing. Chicago: Contemporary Books; 2002. xiii, 318 p. p.

212. Brunvand JH. American folklore : an encyclopedia. New York: Garland Pub.; 1996. xviii, 794 p. p.

213. Duke University. Library. Frank C. Brown Collection of North Carolina Folklore., White NI, Brown FC, North Carolina Folklore Society. The Frank C. Brown Collection of North Carolina Folklore; the folklore of North Carolina, collected by Dr. Frank C. Brown during the years 1912 to 1943, in collaboration with the North Carolina Folklore Society. Durham, N.C.,: Duke University Press; 1952.

214. Puckett NN, Hand WD, Casetta A, Thiederman SB. Popular beliefs and superstitions : a compendium of American folklore : from the Ohio Collection of Newbell Niles Puckett. Boston, Mass.: G.K. Hall; 1981.

215. Browne RB. Popular beliefs and practices from Alabama. Berkeley,: University of California Press; 1958 . 271 p. p.

216. Shelton F. Pioneer comforts and kitchen remedies;: Oldtimey highland secrets from the Blue Ridge and Great Smoky Mountains: Hutcraft; 1965. 24 p.

217. Crellin JK, Philpott J, Bass ALT. Herbal medicine past and present. Durham: Duke University Press; 1990. 218. Krochmal A, Walters RS, Doughty RM. A guide to medicinal plants of Appalachia. Washington,: U.S. Forest Service; for sale by the Supt. of Docs.; 1971. 291 p. p.

219. Stephenson SL. A natural history of the central Appalachians. 1st ed. Morgantown: West Virginia University Press; 2013. xi, 259 p. p.

220. Chrubasik S, Kunzel O, Black A, Conradt C, Kerschbaumer F. Potential economic impact of using a proprietary willow bark extract in outpatient treatment of low back pain: an open non-randomized study. Phytomedicine. 2001;8(4):241-51. doi: 10.1078/0944-7113-00048. PubMed PMID: 11515713.

221. Chrubasik S, Eisenberg E, Balan E, Weinberger T, Luzzati R, Conradt C. Treatment of low back pain exacerbations with willow bark extract: a randomized double-blind study. The American journal of medicine. 2000;109(1):9-14. PubMed PMID: 10936472.

222. Schmid B, Ludtke R, Selbmann HK, Kotter I, Tschirdewahn B, Schaffner W, et al. Efficacy and tolerability of a standardized willow bark extract in patients with osteoarthritis: randomized placebo-controlled, double blind clinical trial. Phytother Res. 2001;15(4):344-50. PubMed PMID: 11406860.

223. World Health Organization. WHO monographs on selected medicinal plants. Geneva: World Health Organization; 1999-2009. v. <1-4> p.

224. Hyson MI. Anticephalgic photoprotective premedicated mask. A report of a successful double-blind placebocontrolled study of a new treatment for headaches with associated frontalis pain and photophobia. Headache.

1998;38(6):475-7. PubMed PMID: 9664755. 
225. Organization WH. WHO Monographs on Slected Medicinal Plants. 2003.

226. Carle R GK. Chamomile: a pharmacological and clinical profile. Drugs of Today. 1992;28:559-65.

227. Gould L RC, Gomprecht RF. Cardiac effect of chamomile tea. Journal of clinical pharmacology. 1973;13:475-9. 228. Chadwick LR, Pauli GF, Farnsworth NR. The pharmacognosy of Humulus lupulus L. (hops) with an emphasis on estrogenic properties. Phytomedicine : international journal of phytotherapy and phytopharmacology. 2006;13(12):11931. doi: 10.1016/j.phymed.2004.07.006. PubMed PMID: PMC1852439.

229. al. LKe. Effects of Humulus lupulus extract on the central nervous system in mice. Planta medica. 1993;59(Suppl.):A691.

230. al. LKe. Neuropharmacological activity of Humulus lupulus extracts. Korean Journal of Pharmacognosy. 1993;24:231-4.

231. Belch JJ, Ansell D, Madhok R, O'Dowd A, Sturrock RD. Effects of altering dietary essential fatty acids on requirements for non-steroidal anti-inflammatory drugs in patients with rheumatoid arthritis: a double blind placebo controlled study. Annals of the rheumatic diseases. 1988;47(2):96-104.

232. Ghazanfar SA. Handbook of Arabian medicinal plants. Boca Raton: CRC Press; 1994. 265 p. p.

233. Srivastava KC, Mustafa T. Ginger (Zingiber officinale) in rheumatism and musculoskeletal disorders. Medical hypotheses.39(4):342-8. doi: 10.1016/0306-9877(92)90059-L.

234. Gobel H, Schmidt G, Soyka D. Effect of peppermint and eucalyptus oil preparations on neurophysiological and experimental algesimetric headache parameters. Cephalalgia : an international journal of headache. 1994;14(3):228-34; discussion 182. PubMed PMID: 7954745.

235. Gobel H, Schmidt G, Dworschak M, Stolze H, Heuss D. Essential plant oils and headache mechanisms. Phytomedicine. 1995;2(2):93-102. doi: 10.1016/S0944-7113(11)80053-X. PubMed PMID: 23196150.

236. Zamora R, Vodovotz Y, Billiar TR. Inducible nitric oxide synthase and inflammatory diseases. Molecular medicine. 2000;6(5):347-73. PubMed PMID: PMC1949959.

237. Pepys MB, Hirschfield GM. C-reactive protein: a critical update. The Journal of clinical investigation. 2003;111(12):1805-12. doi: 10.1172/JCI18921. PubMed PMID: 12813013; PubMed Central PMCID: PMC161431. 238. Szalai AJ. The biological functions of C-reactive protein. Vascular pharmacology. 2002;39(3):105-7. PubMed PMID: 12616974.

239. Szalai AJ, McCrory MA, Cooper GS, Wu J, Kimberly RP. Association between baseline levels of C-reactive protein (CRP) and a dinucleotide repeat polymorphism in the intron of the CRP gene. Genes and immunity. 2002;3(1):149. doi: 10.1038/sj.gene.6363820. PubMed PMID: 11857055.

240. Bucova M, Bernadic M, Buckingham T. C-reactive protein, cytokines and inflammation in cardiovascular diseases. Bratislavske lekarske listy. 2008;109(8):333-40. PubMed PMID: 18837239.

241. Mascolo N, Autore G, Capasso F, Menghini A, Fasulo MP. - Biological screening of Italian medicinal plants for antiinflammatory activity. 1987;- 1(- 1):- 31.

242. al. TBe. Taraxacum offi cinale W.: Pharmacological effect of an ethanol extract. Pharmacology Research. 1993;27(Suppl.1):23-4.

243. Yasukawa K, Yamaguchi A, Arita J, Sakurai S, Ikeda A, Takido M. - Inhibitory effect of edible plant extracts on 12O-tetradecanoylphorbol-13-acetate-induced ear oedema in mice. 1993;- 7(- 2):- 189.

244. Delaveau P, Lallouette P, Tessier AM. [Stimulation of the phagocytic activity of R.E.S. by plant extracts (author's transl)]. Planta medica. 1980;40(1):49-54. doi: 10.1055/s-2008-1074941. PubMed PMID: 6999511.

245. Yesilada E, Ustun O, Sezik E, Takaishi Y, Ono Y, Honda G. Inhibitory effects of Turkish folk remedies on inflammatory cytokines: interleukin-1alpha, interleukin-1beta and tumor necrosis factor alpha. J Ethnopharmacol. 1997;58(1):59-73. PubMed PMID: 9324006.

246. al. WHe. In vitro inhibition of prostaglandin biosynthesis by essential oils and phenolic compounds. Plant Medica. 1986;3:184-7.

247. Yasukawa K, Takeuchi M, Takido M. Humulon, a bitter in the hop, inhibits tumor promotion by 12Otetradecanoylphorbol-13-acetate in two-stage carcinogenesis in mouse skin. Oncology. 1995;52(2):156-8. PubMed PMID: 7854777.

248. H. H. Economic and medicinal plant research. Wagner H HH, Farnsworth NR, editor. London: Academic Press; 1985.

249. Duwiejua M, Zeitlin IJ, Waterman PG, Gray AI. Anti-inflammatory activity of Polygonum bistorta, Guaiacum 
officinale and Hamamelis virginiana in rats. The Journal of pharmacy and pharmacology. 1994;46(4):286-90. PubMed PMID: 8051612.

250. Cuellar MJ, Giner RM, Recio MC, Manez S, Rios JL. Topical anti-inflammatory activity of some Asian medicinal plants used in dermatological disorders. Fitoterapia. 2001;72(3):221-9. PubMed PMID: 11295297. 251. Butenko IG, Gladtchenko SV, Galushko SV. Anti-inflammatory properties and inhibition of leukotriene C4 biosynthesis in vitro by flavonoid baicalein from Scutellaria baicalensis georgy roots. Agents and actions. 1993;39 Spec No:C49-51. PubMed PMID: 8273583.

252. Kimura Y, Yokoi K, Matsushita N, Okuda H. Effects of flavonoids isolated from scutellariae radix on the production of tissue-type plasminogen activator and plasminogen activator inhibitor-1 induced by thrombin and thrombin receptor agonist peptide in cultured human umbilical vein endothelial cells. The Journal of pharmacy and pharmacology. 1997;49(8):816-22. Epub 1997/08/01. PubMed PMID: 9379363.

253. al. URe. American herbal pharmacopeia. Santa Cruz, CA: American Herbal Pharmacopeia; 1999.

254. Wagner I, Greim C, Laufer S, Heide L, Gleiter CH. Influence of willow bark extract on cyclooxygenase activity and on tumor necrosis factor alpha or interleukin 1 beta release in vitro and ex vivo. Clin Pharmacol Ther. 2003;73(3):272-4. Epub 2003/03/07. doi: 10.1067/mcp.2003.32. PubMed PMID: 12621392.

255. Chung CP, Park JB, Bae KH. Pharmacological effects of methanolic extract from the root of Scutellaria baicalensis and its flavonoids on human gingival fibroblast. Planta medica. 1995;61(2):150-3. Epub 1995/04/01. doi: 10.1055/s-2006-958036. PubMed PMID: 7753922.

256. Cheng GF, Liu DP, Yang DX, He KQ, Bai JY, Zhu XY. Antiinflammatory effects of Tremulacin, a Salicinrelated substance isolated from Populus tomentosa Carr. leaves. Phytomedicine. 1994;1(3):209-11. doi: 10.1016/S09447113(11)80067-X. PubMed PMID: 23195941.

257. Srivastava KC. Aqueous extracts of onion, garlic and ginger inhibit platelet aggregation and alter arachidonic acid metabolism. Biomed Biochim Acta. 1984;43(8-9):S335-46. PubMed PMID: 6440548.

258. Mustafa T SK, Jensen KB. Drug development report 9. Pharmacology of ginger, Zingiber officinale. Journal of Drug Development. 1993;6:25-39.

259. Suekawa M, Yuasa K, Isono M, Sone H, Ikeya Y, Sakakibara I, et al. [Pharmacological studies on ginger. IV. Effect of (6)-shogaol on the arachidonic cascade]. Nihon yakurigaku zasshi Folia pharmacologica Japonica. 1986;88(4):263-9. Epub 1986/10/01. PubMed PMID: 3098654.

260. Sharma JN, Srivastava KC, Gan EK. Suppressive effects of eugenol and ginger oil on arthritic rats. Pharmacology. 1994;49(5):314-8. PubMed PMID: 7862743.

261. Shunro K, Morimitsu Y, Toshihiko O. Chemistry of Ginger Components and Inhibitory Factors of the Arachidonic Acid Cascade. Food Phytochemicals for Cancer Prevention II. ACS Symposium Series. 547: American Chemical Society; 1994. p. 244-50.

262. Wang M, Guilbert LJ, Ling L, Li J, Wu Y, Xu S, et al. Immunomodulating activity of CVT-E002, a proprietary extract from North American ginseng (Panax quinquefolium). The Journal of pharmacy and pharmacology. 2001;53(11):1515-23. PubMed PMID: 11732754.

263. Assinewe VA, Amason JT, Aubry A, Mullin J, Lemaire I. Extractable polysaccharides of Panax quinquefolius L. (North American ginseng) root stimulate TNFalpha production by alveolar macrophages. Phytomedicine. 2002;9(5):398404. PubMed PMID: 12222658.

264. Leslie GB. A pharmacometric evaluation of nine Bio-Strath herbal remedies. Medita. 1978;8:3-19.

265. Cebo B et al. Pharmacological properties of saponin fractions obtained from domestic crude drugs: Saponaria officinalis, Primula officinalis and Aesculus hippocastanum. Herba Polonica. 1976;22:154-62.

266. Bruneton, J. Pharmacognosy, phytochemistry, medicinal plants. Paris: Lavoisier; 1995.

267. Wichtl, M. Max Wichtl's herbal drugs \& phytopharmaceuticals. Boca Raton, FL: CRC Press; 1994.

268. Bauer R WH. Echinacea species as potential immunostimulatory drugs. Economic and medicinal plants research. London: Academic Press; 1991. p. 253-321.

269. Arafa NM, Hamuda HM, Melek ST, Darwish SK. The effectiveness of Echinacea extract or composite glucosamine, chondroitin and methyl sulfonyl methane supplements on acute and chronic rheumatoid arthritis rat model. Toxicology and industrial health. 2013;29(2):187-201. doi: 10.1177/0748233711428643. PubMed PMID: 
22173958. 270. Wang H, Guo Y, Zhao X, Li H, Fan G, Mao H, et al. An estrogen receptor dependent mechanism of Oroxylin A in the repression of inflammatory response. PLoS One. 2013;8(7):e69555. doi:

10.1371/journal.pone.0069555. PubMed PMID: 23922737; PubMed Central PMCID: PMC3726624.

271. NIH. Thunder God Vine 2012 [cited 2015 May 23]. Available from: https://nccih.nih.gov/health/tgvine. 272.

Peana AT, D'Aquila PS, Panin F, Serra G, Pippia P, Moretti MD. Anti-inflammatory activity of linalool and linalyl acetate constituents of essential oils. Phytomedicine. 2002;9(8):721-6. doi:

10.1078/094471102321621322. PubMed PMID: 12587692.

273. Halliwell B ZK, Whiteman M The gastrointestinal tract: a major site of antioxidant action? Free Radical Res. 2000;33:819-30.

274. Kratchanova M, Denev P, Ciz M, Lojek A, Mihailov A. Evaluation of antioxidant activity of medicinal plants containing polyphenol compounds. Comparison of two extraction systems. Acta biochimica Polonica. 2010;57(2):229-34. PubMed PMID: 20532255.

275. Ciz M CH, Denev P, Kratchanova M, Slavov A, Lojek A Different methods for control and comparison of the antioxidant properties of vegetables. Food Control. 2010;21:518-23.

276. Denev P LA, Ciz M, Kratchanova M Antioxidant activity and polyphenol content of Bulgarian fruits. Bulgarian Journal of Agricultural Sciences. 2013;19:22-7.

277. Denev P, Kratchanova M, Ciz M, Lojek A, Vasicek O, Blazheva D, et al. Antioxidant, antimicrobial and neutrophilmodulating activities of herb extracts. Acta biochimica Polonica. 2014;61(2):359-67. PubMed PMID: 24945135.

278. Suzuki H, Nishizawa T, Tsugawa H, Mogami S, Hibi T. Roles of oxidative stress in stomach disorders. J Clin Biochem Nutr. 2012;50(1):35-9. doi: 10.3164/jcbn.11-115SR. PubMed PMID: 22247598; PubMed Central PMCID: PMC3246180.

279. Popovic Z, Matic R, Bojovic S, Stefanovic M, Vidakovic V. Ethnobotany and herbal medicine in modern complementary and alternative medicine: An overview of publications in the field of I\&C medicine 2001-2013. J Ethnopharmacol. 2016;181:182-92. doi: 10.1016/j.jep.2016.01.034. PubMed PMID: 26807912.

280. Wu CH, Wang CC, Kennedy J. Changes in herb and dietary supplement use in the U.S. adult population: a comparison of the 2002 and 2007 National Health Interview Surveys. Clin Ther. 2011;33(11):1749-58. doi: 10.1016/j.clinthera.2011.09.024. PubMed PMID: 22030445.

281. Bruni F. Dr. Does-It-All: The New York Times; April 16, 2010. Available from: http://www.nytimes.com/2010/04/18/magazine/180z-t.html?_r=0.

282. Meeting NIoHMPC. Current state of the science to help NCCIH set next 5 yr strategic objectives. In: Feinberg T, editor. webinar2015.

283. Metyas SK, Solyman JS, Arkfeld DG. Inflammatory Fibromyalgia: Is It Real? Curr Rheumatol Rev. 2015. PubMed PMID: 26002453.

284. Bazzichi LF, Rossi AF, Massimetti GF, Giannaccini GF, Giuliano T Fau DF, De Feo FF, et al. Cytokine patterns in fibromyalgia and their correlation with clinical manifestations. 2007.

285. Irwin MR, Olmstead R, Carroll JE. Sleep Disturbance, Sleep Duration, and Inflammation: A Systematic Review and Meta-Analysis of Cohort Studies and Experimental Sleep Deprivation. Biological psychiatry. 2015. doi: 10.1016/j.biopsych.2015.05.014. PubMed PMID: 26140821.

286. Consoli G, Marazziti D, Ciapparelli A, Bazzichi L, Massimetti G, Giacomelli C, et al. The impact of mood, anxiety, and sleep disorders on fibromyalgia. Comprehensive psychiatry. 2012;53(7):962-7. doi: http://dx.doi.org/10.1016/j.comppsych.2012.03.008.

287. Okifuji A, Donaldson GW, Barck L, Fine PG. Relationship between fibromyalgia and obesity in pain, function, mood, and sleep. The journal of pain : official journal of the American Pain Society. 2010;11(12):1329-37. doi: 10.1016/j.jpain.2010.03.006. PubMed PMID: 20542742; PubMed Central PMCID: PMCPMC2939916.

288. Dell'Osso L, Bazzichi L, Baroni S, Falaschi V, Conversano C, Carmassi C, et al. The inflammatory hypothesis of mood spectrum broadened to fibromyalgia and chronic fatigue syndrome. Clinical and experimental rheumatology. 2015;33 Suppl 88(1):109-16. PubMed PMID: 25786052.

289. Okifuji A, Bradshaw DH, Olson C. Evaluating obesity in fibromyalgia: neuroendocrine biomarkers, symptoms, and functions. Clinical rheumatology. 2009;28(4):475-8. doi: 10.1007/s10067-009-1094-2. PubMed PMID: 19172342; PubMed Central PMCID: PMCPMC2668698. 
290. Xiao Y Fau - Haynes WL, Fau HWL, Fau MJE, Russell IJ. Elevated serum high-sensitivity C-reactive protein levels in fibromyalgia syndrome patients correlate with body mass index, interleukin-6, interleukin-8, erythrocyte sedimentation rate. 2013.

291. Frisbee SJ, Brooks AP, Jr., Maher A, Flensborg P, Arnold S, Fletcher T, et al. The C8 health project: design, methods, and participants. Environmental health perspectives. 2009;117(12):1873-82. Epub 2010/01/06. doi: 10.1289/ehp.0800379. PubMed PMID: 20049206; PubMed Central PMCID: PMCPMC2799461.

292. Martinez-Jauand M, Sitges C, Femenia J, Cifre I, Gonzalez S, Chialvo D, et al. Age-of-onset of menopause is associated with enhanced painful and non-painful sensitivity in fibromyalgia. Clinical rheumatology. 2013;32(7):97581. doi: 10.1007/s10067-013-2212-8. PubMed PMID: 23417348.

293. Goesling J, Brummett CM, Meraj TS, Moser SE, Hassett AL, Ditre JW. Associations Between Pain, Current Tobacco Smoking, Depression, and Fibromyalgia Status Among Treatment-Seeking Chronic Pain Patients. Pain Med. 2015;16(7):1433-42. doi: 10.1111/pme.12747. PubMed PMID: 25801019.

294. Prasad K. C-reactive protein (CRP)-lowering agents. Cardiovascular drug reviews. 2006;24(1):33-50. Epub 2006/08/31. doi: 10.1111/j.1527-3466.2006.00033.x. PubMed PMID: 16939632.

295. NIH. Classification of Overweight and Obesity by BMI, Waist Circumference, and Associated Disease Risks: National Heart, Lung, and Blood Institute

[cited 2015 February 7]. Available from: https://www.nhlbi.nih.gov/health/educational/lose_wt/BMI/bmi_dis.htm.

296. Deodhar AA, Fisher RA, Blacker CV, Woolf AD. Fluid retention syndrome and fibromyalgia. Br J Rheumatol. 1994;33(6):576-82. PubMed PMID: 8205408.

297. Mork PJ, Vasseljen O, Nilsen TI. Association between physical exercise, body mass index, and risk of fibromyalgia: longitudinal data from the Norwegian Nord-Trondelag Health Study. Arthritis Care Res (Hoboken). 2010;62(5):611-7. doi: 10.1002/acr.20118. PubMed PMID: 20191480.

298. Sanchez del Rio-Gonzalez M. Chronic migraine: pathophysiology. Rev Neurol. 2012;54 Suppl 2:S13-9. PubMed PMID: 22532238.

299. Lawrence R, Felson D, Helmick CG, Arnold LM, Choi H, Deyo RA, et al. Estimates of the prevalence of arthritis and other rheumatic conditions in the United States. Part II. (0004-3591 (Print)). doi: D - NLM: NIHMS347693 D - NLM: PMC3266664 EDAT- 2008/01/01 09:00 MHDA- 2008/03/11 09:00 CRDT2008/01/01 09:00 AID - 10.1002/art.23176 [doi] PST - ppublish.

300. White KP, Speechley M, Harth M, Ostbye T. The London Fibromyalgia Epidemiology Study: the prevalence of fibromyalgia syndrome in London, Ontario. J Rheumatol. 1999;26(7):1570-6. Epub 1999/07/16. PubMed PMID: 10405947.

301. Schochat T, Beckmann C. Sociodemographic characteristics, risk factors and reproductive history in subjects with fibromyalgia-results of a population-based case-control study. Zeitschrift fur Rheumatologie. 2003;62(1):46-59. doi: 10.1007/s00393-003-0447-5. PubMed PMID: 12624804.

302. Wright LJ, Schur E, Noonan C, Ahumada S, Buchwald D, Afari N. Chronic pain, overweight, and obesity: findings from a community-based twin registry. The journal of pain : official journal of the American Pain Society. 2010;11(7):628-35. doi: 10.1016/j.jpain.2009.10.004. PubMed PMID: 20338816; PubMed Central PMCID: PMCPMC2892725.

303. Masters ET, Mardekian J, Emir B, Clair A, Kuhn M, Silverman SL. Electronic medical record data to identify variables associated with a fibromyalgia diagnosis: importance of health care resource utilization. J Pain Res. 2015;8:1318. doi: 10.2147/JPR.S74900. PubMed PMID: 25784819; PubMed Central PMCID: PMCPMC4356680.

304. Di Franco M, Iannuccelli C, Bazzichi L, Atzeni F, Consensi A, Salaffi F, et al. Misdiagnosis in fibromyalgia: a multicentre study. Clinical and experimental rheumatology. 2011;29(6 Suppl 69):S104-8. PubMed PMID: 22243557. 305. Wolfe F, Clauw Dj Fau - Fitzcharles M-A, Fitzcharles Ma Fau - Goldenberg DL, Goldenberg D1 Fau - Hauser W, Hauser W Fau - Katz RS, Katz Rs Fau - Mease P, et al. Fibromyalgia criteria and severity scales for clinical and epidemiological studies: a modification of the ACR Preliminary Diagnostic Criteria for Fibromyalgia. Arthritis Care \& Research: American College of Rheumatology; 2010. p. 600-10.

306. Choi HJ, Han JY, Seo MR, Ryu HJ, Baek HJ. Fibromyalgia with chronic rheumatic diseases in South Korea: a comparison of clinical and American College of Rheumatology criteria. International journal of rheumatic diseases. 2015. doi: 10.1111/1756-185X.12678. PubMed PMID: 25990794.

307. Calandre EP, Rico-Villademoros F, Slim M. An update on pharmacotherapy for the treatment of fibromyalgia. 
Expert Opin Pharmacother. 2015;16(9):1347-68. doi: 10.1517/14656566.2015.1047343. PubMed PMID:

26001183. 308. NIH. Pain: Considering Complementary Approaches (eBook) [cited 2016]. Available from: https://nccih.nih.gov/health/pain/ebook.

309. Centers for Disease Control and Prevention. NHIS Survey Description. In: Statistics DoHISNCfH, editor. Hyattsville, MD, December 2003.

310. Centers for Disease Control and Prevention USDoHaHS. 2012 National Health Interview Survey (NHIS)

Public Use Data Release. In: Statistics DoHI, Statistics NCfH, editors. Hyattsville, Maryland: Centers for Disease Control and Prevention U.S. Department of Health and Human Services; 2013.

311. Wahner-Roedler DL, Thompson JM, Luedtke CA, King SM, Cha SS, Elkin PL, et al. Dietary soy supplement on fibromyalgia symptoms: a randomized, double-blind, placebo-controlled, early phase trial. Evidence-based complementary and alternative medicine : eCAM. 2011;2011:350697. doi: 10.1093/ecam/nen069. PubMed PMID: 18990724; PubMed Central PMCID: PMCPMC3136370.

312. FDA. FDA Approves First Drug for Treating Fibromyalgia: U.S. Department of Health and Human Services; 2007. Available from: http://www.fda.gov/NewsEvents/Newsroom/PressAnnouncements/2007/ucm108936.htm. 313. Peregoy JA, Clarke TC, Jones LI, Stussman BJ, Nahin RL. Regional variation in use of complementary health approaches by U.S. adults. NCHS Data Brief. 2014(146):1-8. PubMed PMID: 24750666.

314. Classification of chronic pain. Descriptions of chronic pain syndromes and definitions of pain terms. Prepared by the International Association for the Study of Pain, Subcommittee on Taxonomy. Pain Supplement. 1986;3:S1-226. PubMed PMID: 3461421.

315. Gentile DA, Woodhouse J, Lynch P, Maier J, McJunkin T. Reliability and Validity of the Global Pain Scale with Chronic Pain Sufferers. Pain Physician. 2011;14:61-70.

316. Tyson Sf BP. How to measure pain in neurological conditions? A systematic review of psychometric properties and clinical utility of measurement tools. Clinical Rehabilitation. 2013.

317. Singh JA, Luo R Fau - Landon GC, Landon Gc Fau - Suarez-Almazor M, Suarez-Almazor M. Reliability and Clinically Important Improvement Thresholds for Osteoarthritis Pain and Function Scales: A Multicenter Study.

318. Salaffi F, Sarzi-Puttini P, Ciapetti A, Atzeni F. Assessment instruments for patients with fibromyalgia: properties, applications and interpretation. Clinical and experimental rheumatology. 2009;27(5 Suppl 56):S92-105. PubMed PMID: 20074447.

319. Reed MD, Van Nostran W. Assessing pain intensity with the visual analogue scale: A plea for uniformity. The Journal of Clinical Pharmacology. 2013:n/a-n/a. doi: 10.1002/jcph.250.

320. Plan EL, Elshoff Jp Fau - Stockis A, Stockis A Fau - Sargentini-Maier ML, Sargentini-Maier Ml Fau - Karlsson MO, Karlsson MO. Likert pain score modeling: a Markov integer model and an autoregressive continuous model. 321. Lukas A, Barber Jb Fau - Johnson P, Johnson P Fau - Gibson SJ, Gibson SJ. Observer-rated pain assessment instruments improve both the detection of pain and the evaluation of pain intensity in people with dementia. 322. Liu JYW, Briggs M, Closs SJ. The Psychometric Qualities of Four Observational Pain Tools (OPTs) for the Assessment of Pain in Elderly People with Osteoarthritic Pain. Journal of pain and symptom management.

2010;40(4):582-98. doi: http://dx.doi.org/10.1016/j.jpainsymman.2010.02.022.

323. Hawker GA, Mian S, Kendzerska T, French M. Measures of adult pain: Visual Analog Scale for Pain (VAS Pain), Numeric Rating Scale for Pain (NRS Pain), McGill Pain Questionnaire (MPQ), Short-Form McGill Pain Questionnaire (SF-MPQ), Chronic Pain Grade Scale (CPGS), Short Form-36 Bodily Pain Scale (SF-36 BPS), and Measure of Intermittent and Constant Osteoarthritis Pain (ICOAP). Arthritis Care \& Research.

2011;63(S11):S240-S52. doi: 10.1002/acr.20543.

324. Gelinas C, Puntillo Ka Fau - Joffe AM, Joffe Am Fau - Barr J, Barr J. A validated approach to evaluating psychometric properties of pain assessment tools for use in nonverbal critically ill adults.

325. Gendreau M, Hufford MR, Stone AA. Measuring clinical pain in chronic widespread pain: selected methodological issues. Chronic Generalised Musculoskeletal Pain. 2003;17(4):575-92. doi: http://dx.doi.org/10.1016/S15216942(03)00031-7.

326. Carter GT, Duong V, Ho S, Ngo KC, Greer CL, Weeks DL. Side effects of commonly prescribed analgesic medications. Physical medicine and rehabilitation clinics of North America. 2014;25(2):457-70. doi: 10.1016/j.pmr.2014.01.007. PubMed PMID: 24787343. 
327. Centers for Disease Control and Prevention USDoHaHS. Drug Overdose Deaths by State, US 2013 and 20142015 [updated Dec 18, 2015]. Available from: http://www.cdc.gov/drugoverdose/data/statedeaths.html.

328. Herman PM, Poindexter BL, Witt CM, Eisenberg DM. Are complementary therapies and integrative care costeffective? A systematic review of economic evaluations. BMJ open. 2012;2(5). doi: 10.1136/bmjopen-2012001046. PubMed PMID: 22945962; PubMed Central PMCID: PMCPMC3437424.

329. Santaguida P, Gross A, United States. Agency for Healthcare Research and Quality., McMaster University. Evidence-based Practice Center. Complementary and alternative medicine in back pain utilization report. Rockville, MD: Agency for Healthcare Research and Quality; 2009. ix, 149 p. p.

330. CDC. State-Specific 2013 BRFSS Prevalence Estimates: Arthritis Prevalence Estimates by State. Available from: http://www.cdc.gov/arthritis/data_statistics/state-data-current.htm.

331. Weinstein ND, Rothman AJ, Sutton SR. Stage theories of health behavior: conceptual and methodological issues. Health Psychology. 1998;17(3):290-9.

332. Prochaska JO, DiClemente CC, Norcross JC. In search of how people change: applications to addictive behaviors. American Psychologist. 1992;47(9):1102-14.

333. Prochaska JO, Norcross JC, DiClemente CC. Changing for good : the revolutionary program that explains the six stages of change and teaches you how to free yourself from bad habits. 1st ed. New York: W. Morrow; 1994. 304 p. p.

334. Prochaska JO. Decision making in the transtheoretical model for behavior change. . Medical Decision Making. 2008;28(6):845-9.

335. Di Noia J, Prochaska JO. Dietary stages of change and decisional balance: a meta-analytic review. American Journal of Health Behavior. 2010;34(5):618-32.

336. Plotnikoff GA, Quigley JM. Prevalence of Severe Hypovitaminosis D in Patients With Persistent, Nonspecific Musculoskeletal Pain. Mayo Clinic proceedings. 2003;78(12):1463-70. doi: http://dx.doi.org/10.4065/78.12.1463. 337. Jezewski MA, Finnell DS, Wu Y-WB, Meeker MA, Sessanna L, Lee J. Psychometric testing of four transtheoretical model questionnaires for the behavior, completing health care proxies. Research in nursing \& health. 2009;32(6):606-20. doi: 10.1002/nur.20352.

338. Henchoz Y, Zufferey P, So A. Stages of change, barriers, benefits, and preferences for exercise in RA patients: a crosssectional study. Scand J Rheumatol. 2013;42(2):136-45. doi: 10.3109/03009742.2012.724707. PubMed PMID: 23244196.

339. Johnson P, Fallon Ea Fau - Harris BS, Harris Bs Fau - Burton B, Burton B. Body satisfaction is associated with Transtheoretical Model constructs for physical activity behavior change.

340. Choi JH, Chung Km Fau - Park K, Park K. Psychosocial predictors of four health-promoting behaviors for cancer prevention using the stage of change of Transtheoretical Model. LID - 10.1002/pon.3278 [doi].

341. Glanz K, Rimer B, Viswanath K. Health Behavior and Health Education: Theory, Research, and Practice. San Francisco, CA: Jossey-Bass; 2008.

342. Perrin KM. Essentials of planning and evaluation for public health. Burlington, MA: Jones \& Bartlett Learning; 2016. xvi, 254 pages $p$.

343. Plotnikoff RC, Lippke S, Johnson ST, Hotz SB, Birkett NJ, Rossi SR. Applying the stages of change to multiple lowfat dietary behavioral contexts. An examination of stage occupation and discontinuity. Appetite. 2009;53(3):345-53. doi: http://dx.doi.org.www.libproxy.wvu.edu/10.1016/j.appet.2009.07.016.

344. Picciano MF, Dwyer JT, Radimer KL, Wilson DH, Fisher KD, Thomas PR, et al. Dietary supplement use among infants, children, and adolescents in the United States, 1999-2002. Arch Pediatr Adolesc Med. 2007;161(10):978-85. doi: 10.1001/archpedi.161.10.978. PubMed PMID: 17909142.

345. Zhang Y, Fein EB, Fein SB. Feeding of dietary botanical supplements and teas to infants in the United States. Pediatrics. 2011;127(6):1060-6. doi: 10.1542/peds.2010-2294.

346. Peana AT, Marzocco S, Popolo A, Pinto A. (-)-Linalool inhibits in vitro NO formation: Probable involvement in the antinociceptive activity of this monoterpene compound. Life sciences. 2006;78(7):719-23. doi: 10.1016/j.lfs.2005.05.065. PubMed PMID: 16137709.

347. Peana AT, Rubattu P, Piga GG, Fumagalli S, Boatto G, Pippia P, et al. Involvement of adenosine A1 and A2A receptors in (-)-linalool-induced antinociception. Life sciences. 2006;78(21):2471-4. doi: 10.1016/j.lfs.2005.10.025. PubMed PMID: 16343551. 
348. Re L, Barocci S, Sonnino S, Mencarelli A, Vivani C, Paolucci G, et al. Linalool modifies the nicotinic receptorion channel kinetics at the mouse neuromuscular junction. Pharmacol Res. 2000;42(2):177-82. doi: 10.1006/phrs.2000.0671. PubMed PMID: 10887049.

349. Grossman M. The demand for health: a theoretical and empirical investigation. New York,: National Bureau of Economic Research; distributed by Columbia University Press; 1972. xvii, 115 p. p. 